\title{
Cobalt(III)-Catalyzed Intermolecular Carboamination of Propiolates and Bicyclic alkenes via Non-annulative Redox- Neutral Coupling
}

Yuelu Zhu, ${ }^{\text {ac }}$ Feng Chen, ${ }^{\text {a }}$ Xinyang Zhao, ${ }^{\text {a }}$ Dingyuan Yan, ${ }^{a}$ Wanxiong

$$
\text { Yong, }{ }^{\mathrm{b}} \text { and Jing Zhao*ac }
$$

a State Key Laboratory of Coordination Chemistry, Institute of Chemistry and BioMedical Sciences, School of Chemistry and Chemical Engineering, Nanjing University, Nanjing 210093, China.

${ }^{b}$ College of Chemical Engineering, Nanjing Forestry University, Nanjing 210037, China.

c Shenzhen Research Institute, Nanjing University, Shenzhen, 518000, China.

\section{Table of content}

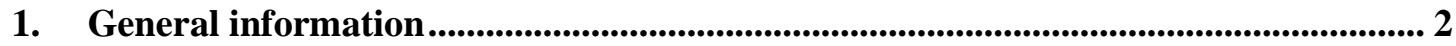

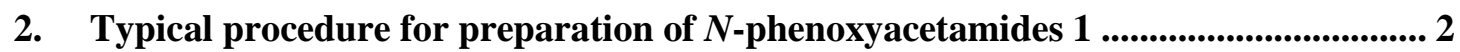

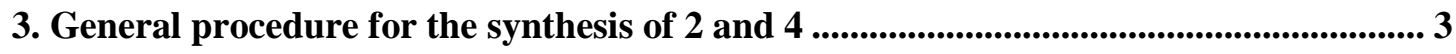

4. Experimental procedure and characterization of products 3 and 5................................. 4

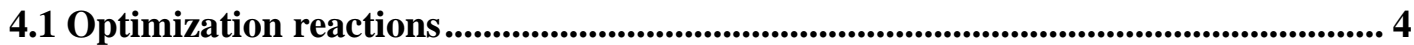

4.2 General procedure for the $\mathrm{Cp} * \mathrm{Co}(\mathrm{III})$-catalyzed synthesis of 3 and 5 ..................... 5

4.3 Gram-Scale Synthesis of 3aa ........................................................................................... 6

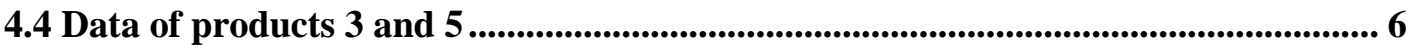

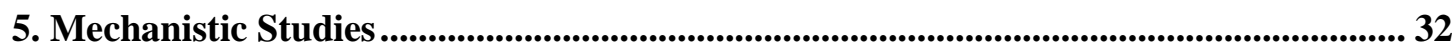

6. Crystallographic data 3am, 3ao, and 5sa ............................................................................... 37

7. Literature ….................................................................................................................................................... 54

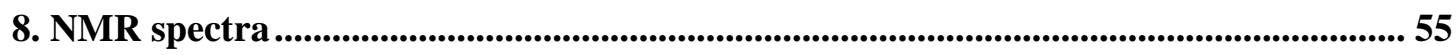




\section{General information}

The reactions were performed under nitrogen atmosphere in oven-dried glassware, unless otherwise noted. Further, the reaction temperatures are reported corresponding to the oil bath temperature. Dry solvents were bought and stored over molecular sieves in nitrogen atmosphere. Commercially available chemicals were obtained from Sigma-Aldrich, Alfa Aesar, Adamas, and Aladdin and used as received unless otherwise stated. Reactions were monitored with analytical thin-layer chromatography (TLC) on silica. Melting points were determined using a standard melting point instrument and are uncorrected. ${ }^{1} \mathrm{H}$ NMR and ${ }^{13} \mathrm{C}$ NMR data were recorded on Bruker nuclear resonance $(400 \mathrm{MHz}$, and 500MHz) spectrometers unless otherwise specified, respectively. Chemical shifts $(\delta)$ are given in ppm relative to TMS. The residual solvent signals were used as references and the chemical shifts converted to the TMS scale. HRMS were conducted on an Agilent 6540Q-TOF LC/MS equipped with an electrospray ionization (ESI) probe operating in positive ion mode

\section{Typical procedure for preparation of $N$-phenoxyacetamides 1}

\section{Method A:}

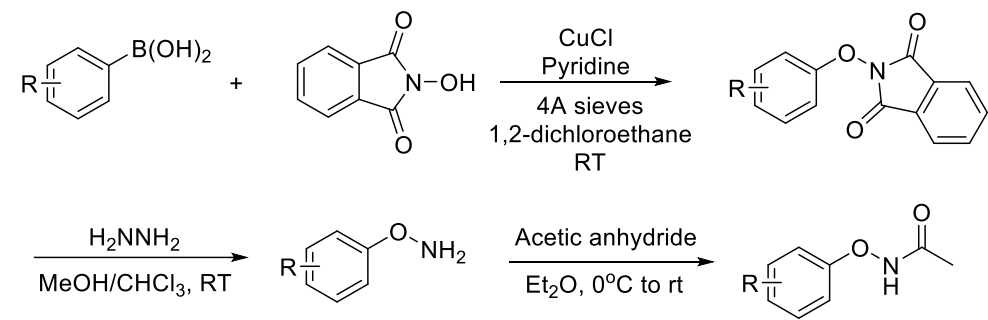

Following a literature reports, ${ }^{[1]}$ in a $50 \mathrm{~mL}$ round-bottom flask, $N$-hydroxyphthalimide (1.0 eq), cooper(I) chloride (1.0 eq), freshly activated $4 \AA$ molecular sieves $(250 \mathrm{mg} / \mathrm{mmol})$, and phenylboronic acid (2.0 eq) were combined in 1,2-dichloroethane $(0.2 \mathrm{M})$. The pyridine (1.1 eq) was then added to the suspension. The reaction mixture was open to the atmosphere and stirred at room temperature over 24-48h. Upon completion, silica gel was added to the flask and the solvent was removed under vacuum. The desired $N$-aryloxyphthalimides were obtained by flash column chromatography on silica gel.

Hydrazine monohydrate (3.0 eq) was added to the solution of $N$-aryloxyphthalimide (1.0 eq) in $10 \% \mathrm{MeOH}$ in $\mathrm{CHCl}_{3}(0.1 \mathrm{M})$. The reaction was allowed to stir at room temperature overnight. Upon completion, the reaction mixture was filtered off and washed with $\mathrm{CH}_{2} \mathrm{Cl}_{2}$. The filtrate was concentrated under reduced pressure, and purified by flash silica gel column chromatography to give the corresponding $N$-aryloxyamine.

In a $20 \mathrm{~mL}$ round-bottom flask, $N$-aryloxyamine $(1.0 \mathrm{eq})$ was dissolved in ether $(0.2 \mathrm{M})$. The flask was cooled in an ice bath, to which acetic anhydride (2.0 eq) was slowly added. The ice bath was allowed to warm to room temperature and the mixture was stirred for $3 \mathrm{~h}$ at room temperature. The reaction mixture was concentrated under reduced pressure and purified by flash silica gel column chromatography to give the corresponding $N$-aryloxyacetamide. 


\section{Method B:}

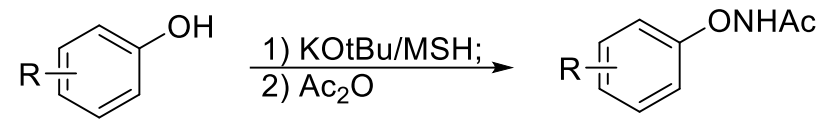

Following a literature reports, ${ }^{[2]}$ phenols $(2.76 \mathrm{mmol})$ was dissolved in $4 \mathrm{~mL}$ of methanol, and then potassium tert-butoxide ( $309 \mathrm{mg}, 2.76 \mathrm{mmol}$ ) was added. The mixture was allowed to stir for $0.5 \mathrm{~h}$ under $\mathrm{N}_{2}$ atmosphere. The methanol was removed, and the residue was taken up in $2 \mathrm{~mL}$ of dichloromethane. Then the freshly prepared O-mesitylsulfonylhydroxylamine (378 $\mathrm{mg}, 1.76 \mathrm{mmol}$ ) in $2 \mathrm{~mL}$ of dichloromethane was added under ice cooling. The mixture was allowed to stir for $1 \mathrm{~h}$, dichloromethane was then removed under reduce pressure to afford the corresponding $N$-aryloxyamine.

\section{General procedure for the synthesis of 2 and 4}

\section{Alkynes 2 were prepared according to the following general procedure ${ }^{[3]}$ :}

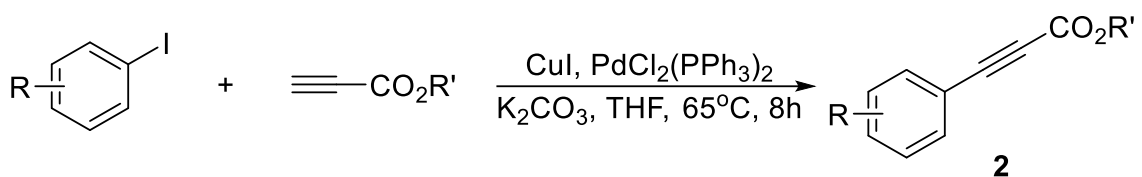

To a solution of aryl iodide ( $7.5 \mathrm{mmol}, 1.5$ equiv), alkyl propiolate ( $445 \mu \mathrm{L}, 5 \mathrm{mmol}, 1.0$ equiv) and $\mathrm{K}_{2} \mathrm{CO}_{3}\left(2.07 \mathrm{~g}, 15 \mathrm{mmol}, 3.0\right.$ equiv) in THF $(40 \mathrm{~mL})$ was added $\mathrm{PdCl}_{2}\left(\mathrm{PPh}_{3}\right)_{2}(70.2$ $\mathrm{mg}, 0.1 \mathrm{mmol}, 2 \mathrm{~mol} \%)$. The mixture was stirred for $5 \mathrm{~min}$. And $\mathrm{CuI}(9.5 \mathrm{mg}, 0.05 \mathrm{mmol}, 1$ mol\%) was added. The resulting mixture was then heated under a nitrogen atmosphere at $65^{\circ} \mathrm{C}$ for $8 \mathrm{~h}$. The mixture was cooled to room temperature, and the solid was removed by filtration. Then the filtrate was concentrated under reduced pressure and the residue was purified by column chromatography on silica gel using a mixture of petroleum ether and ethyl acetate as the eluent (100:1 to $60: 1)$ to provide the desired product.

\section{Alkenes 4 were prepared according to the following general procedure:}

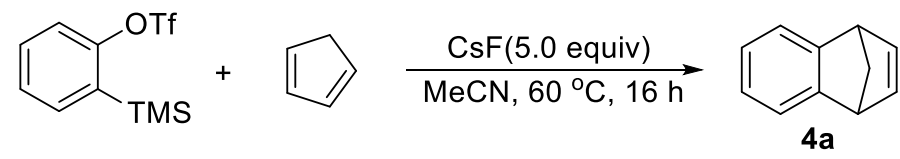

To a stirred solution of silyltriflate $(500 \mathrm{mg}, 1.68 \mathrm{mmol})$ and cyclopentadiene (705 $\mu \mathrm{L}$, 8.38 mmol, 5 equiv) in $\mathrm{CH}_{3} \mathrm{CN}(17 \mathrm{~mL})$ was added $\mathrm{CsF}$ (1.3 g, $8.38 \mathrm{mmol}, 5$ equiv). The reaction vessel was sealed and placed in an aluminum heating block maintained at $60^{\circ} \mathrm{C}$ for 16 h. After cooling to room temperture, the reaction mixture was filtered over silica gel (EtOAc eluent). Evaporation under reduced pressure afforded the crude product. The crude residue was further purified by column chromatography (hexanes) to afford bicyclic olefins $4 \mathbf{a}$ (239 $\mathrm{mg}$, $99 \%$ yield) as a colorless oil. ${ }^{[4]}$

$N$-Ts-bicyclic alkenes $\mathbf{4 b},{ }^{[5]} N$-Ms-bicyclic alkene $\mathbf{4 c},{ }^{[6]} N$-Boc-bicyclic alkene $\mathbf{4 d},{ }^{[7]} \mathbf{4 f}, \mathbf{4 g}$, 
and $\mathbf{4} \mathbf{h}^{[8]}$ are known compounds and were prepared according to the methods given in the cited references without any optimization of the reaction conditions.

\section{Experimental procedure and characterization of products 3 and 5.}

\subsection{Optimization reactions}

Table S1: Condition screening for the $\mathrm{Cp} * \mathrm{Co}(\mathrm{III})$-catalyzed synthesis of $3 \mathrm{aa}^{[\mathrm{aa}}$

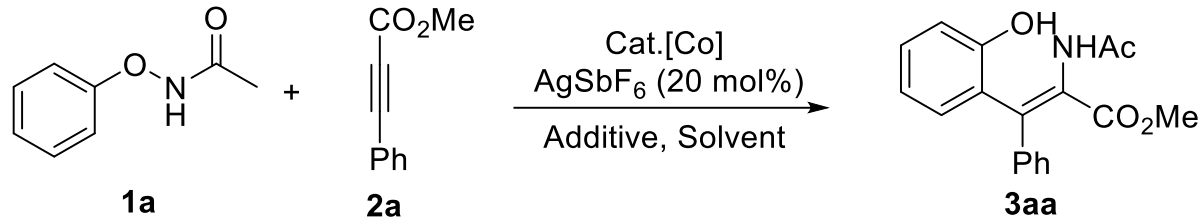

\begin{tabular}{|c|c|c|c|c|c|}
\hline Entry & Cat(10 mol\%) & Additive (equiv) & Solvent & $\mathrm{T}\left({ }^{\circ} \mathrm{C}\right)$ & Yield $^{b}$ \\
\hline 1 & {$\left[\mathrm{Cp} * \mathrm{Co}(\mathrm{CO}) \mathrm{I}_{2}\right]$} & $\mathrm{NaOAc}(0.5)$ & TFE & 50 & $19 \%$ \\
\hline 2 & {$\left[\mathrm{Cp} * \mathrm{Co}(\mathrm{CO}) \mathrm{I}_{2}\right]$} & $\mathrm{CsOAc}(0.5)$ & TFE & 50 & $32 \%$ \\
\hline 3 & {$\left[\mathrm{Cp} * \mathrm{Co}(\mathrm{CO}) \mathrm{I}_{2}\right]$} & KOAc $(0.5)$ & TFE & 50 & $26 \%$ \\
\hline 4 & {$\left[\mathrm{Cp} * \mathrm{Co}(\mathrm{CO}) \mathrm{I}_{2}\right]$} & $\mathrm{K}_{3} \mathrm{PO}_{4}(0.5)$ & TFE & 50 & $57 \%$ \\
\hline 5 & {$\left[\mathrm{Cp} * \mathrm{Co}(\mathrm{CO}) \mathrm{I}_{2}\right]$} & PivOH (0.5) & TFE & 50 & N.R \\
\hline 6 & {$[\mathrm{Cp} * \mathrm{Co}(\mathrm{CO}) \mathrm{I} 2]$} & $\mathrm{AcOH}(0.5)$ & TFE & 50 & N.R \\
\hline 7 & {$\left[\mathrm{Cp} * \mathrm{Co}(\mathrm{CO}) \mathrm{I}_{2}\right]$} & $\mathrm{K}_{3} \mathrm{PO}_{4}(0.5) \mathrm{KOAc}(0.5)$ & TFE & 50 & $71 \%$ \\
\hline 8 & {$\left[\mathrm{Cp} * \mathrm{Co}(\mathrm{CO}) \mathrm{I}_{2}\right]$} & $\mathrm{K}_{3} \mathrm{PO}_{4}(0.5) \mathrm{CsOAc}(0.5)$ & TFE & 50 & $82 \%$ \\
\hline 9 & {$\left[\mathrm{Cp} * \mathrm{Co}(\mathrm{CO}) \mathrm{I}_{2}\right]$} & $\mathrm{K}_{3} \mathrm{PO}_{4}(0.5) \mathrm{CsOAc}(0.5)$ & TFE & 50 & $59 \%$ \\
\hline 10 & {$\left[\mathrm{Cp} * \mathrm{Co}(\mathrm{CO}) \mathrm{I}_{2}\right]$} & $\mathrm{K}_{3} \mathrm{PO}_{4}(0.5) \mathrm{CsOAc}(0.5)$ & TFE & 40 & $86 \%$ \\
\hline 11 & $\mathrm{CoBr}_{2}$ & $\mathrm{~K}_{3} \mathrm{PO}_{4}(0.5) \mathrm{CsOAc}(0.5)$ & TFE & 40 & N.R \\
\hline 12 & $\mathrm{CoCl}_{2}$ & $\mathrm{~K}_{3} \mathrm{PO}_{4}(0.5) \mathrm{CsOAc}(0.5)$ & TFE & 40 & N.R \\
\hline 13 & $\mathrm{Co}(\mathrm{acac})_{2}$ & $\mathrm{~K}_{3} \mathrm{PO}_{4}(0.5) \mathrm{CsOAc}(0.5)$ & TFE & 40 & N.R \\
\hline 14 & & $\mathrm{~K}_{3} \mathrm{PO}_{4}(0.5) \mathrm{CsOAc}(0.5)$ & TFE & 40 & N.R \\
\hline $15^{\mathrm{c}}$ & {$\left[\mathrm{Cp} * \mathrm{Co}(\mathrm{CO}) \mathrm{I}_{2}\right]$} & $\mathrm{K}_{3} \mathrm{PO}_{4}(0.5) \mathrm{CsOAc}(0.5)$ & TFE & 40 & $77 \%$ \\
\hline
\end{tabular}

[a] Reaction conditions: 1a $(0.1 \mathrm{mmol}), \mathbf{2 a}(0.12 \mathrm{mmol})$, catalyst $(10 \mathrm{~mol} \%), \operatorname{AgSbF}_{6}(20$ mol\%), additive $(0.5$ equiv), in a solvent $(0.5 \mathrm{~mL})$ for $24 \mathrm{~h}$. [b] Isolated yield. [c] catalyst (5 $\mathrm{mol} \%)$ 
Table S2: Condition screening for the $\mathrm{Cp} * \mathrm{Co}(\mathrm{III})-$ catalyzed synthesis of 5aa ${ }^{[\mathrm{a}]}$
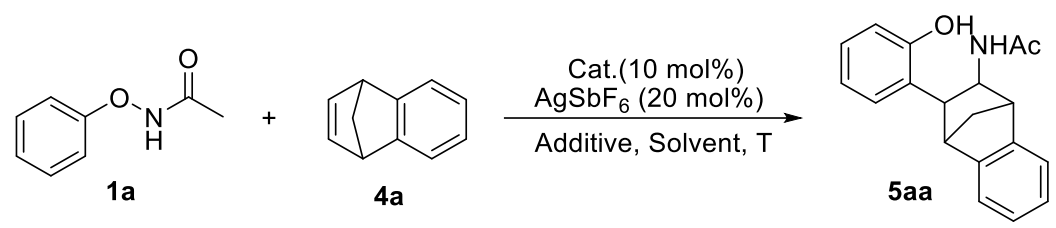

\begin{tabular}{cccccc}
\hline Entry & Catalyst $\left(10 \mathrm{~mol}^{2}\right)$ & Additives (equiv) & Solvent & $\mathrm{T}\left({ }^{\circ} \mathrm{C}\right)$ & Yield $^{\mathrm{b}}$ \\
\hline 1 & {$\left[\mathrm{Cp} * \mathrm{Co}(\mathrm{CO}) \mathrm{I}_{2}\right]$} & $\mathrm{NaOAc}(0.5)$ & $\mathrm{TFE}$ & 50 & $12 \%$ \\
2 & {$\left[\mathrm{Cp} * \mathrm{Co}(\mathrm{CO}) \mathrm{I}_{2}\right]$} & $\mathrm{CsOAc}(0.5)$ & $\mathrm{TFE}$ & 50 & $14 \%$ \\
3 & {$\left[\mathrm{Cp} * \mathrm{Co}(\mathrm{CO}) \mathrm{I}_{2}\right]$} & $\mathrm{KOAc}(0.5)$ & $\mathrm{TFE}$ & 50 & $36 \%$. \\
4 & {$\left[\mathrm{Cp} * \mathrm{Co}(\mathrm{CO}) \mathrm{I}_{2}\right]$} & $\mathrm{K}_{3} \mathrm{PO}_{4}(0.5)$ & $\mathrm{TFE}$ & 50 & $31 \%$ \\
5 & {$\left[\mathrm{Cp} * \mathrm{Co}(\mathrm{CO}) \mathrm{I}_{2}\right]$} & $\mathrm{Cs}_{2} \mathrm{CO}_{3}(0.5)$ & $\mathrm{TFE}$ & 50 & Trace \\
6 & {$\left[\mathrm{Cp} * \mathrm{Co}(\mathrm{CO}) \mathrm{I}_{2}\right]$} & $\mathrm{K}_{2} \mathrm{CO}_{3}(0.5)$ & $\mathrm{TFE}$ & 50 & $18 \%$ \\
7 & {$\left[\mathrm{Cp} * \mathrm{Co}(\mathrm{CO}) \mathrm{I}_{2}\right]$} & $\mathrm{K}_{3} \mathrm{PO}_{4}(0.5) \mathrm{CsOAc}(0.5)$ & $\mathrm{TFE}$ & 50 & $73 \%$. \\
8 & {$\left[\mathrm{Cp} * \mathrm{Co}(\mathrm{CO}) \mathrm{I}_{2}\right]$} & $\mathrm{K}_{3} \mathrm{PO}_{4}(0.5) \mathrm{KOAc}(0.5)$ & $\mathrm{TFE}$ & 50 & $79 \%$ \\
9 & {$\left[\mathrm{Cp} * \mathrm{Co}(\mathrm{CO}) \mathrm{I}_{2}\right]$} & $\mathrm{K}_{3} \mathrm{PO}_{4}(0.5) \mathrm{KOAc}(0.5)$ & $\mathrm{TFE}$ & 40 & $86 \%$ \\
10 & {$\left[\mathrm{Cp} * \mathrm{Co}(\mathrm{CO}) \mathrm{I}_{2}\right]$} & $\mathrm{K}_{3} \mathrm{PO}_{4}(0.5) \mathrm{KOAc}(0.5)$ & $\mathrm{TFE}$ & $\mathrm{RT}$ & $59 \%$ \\
11 & $\mathrm{CoBr}$ & $\mathrm{K}_{3} \mathrm{PO}_{4}(0.5) \mathrm{KOAc}(0.5)$ & $\mathrm{TFE}$ & 40 & $\mathrm{~N} . \mathrm{R}$ \\
12 & $\mathrm{CoCl}$ & $\mathrm{K}_{3} \mathrm{PO}_{4}(0.5) \mathrm{KOAc}(0.5)$ & $\mathrm{TFE}$ & 40 & $\mathrm{~N} . \mathrm{R}$ \\
13 & $\mathrm{Co}(\mathrm{acac})_{2}$ & $\mathrm{~K}_{3} \mathrm{PO}_{4}(0.5) \mathrm{KOAc}(0.5)$ & $\mathrm{TFE}$ & 40 & $\mathrm{~N} . \mathrm{R}$ \\
\hline
\end{tabular}

[a] Reaction conditions: 1a $(0.1 \mathrm{mmol}), \mathbf{4 a}(0.12 \mathrm{mmol})$, catalyst $(10 \mathrm{~mol} \%), \operatorname{AgSbF}_{6}(20$ mol\%), additive ( 0.5 equiv), in a solvent $(0.5 \mathrm{~mL})$ for $24 \mathrm{~h}$. [b] Isolated yield.

\subsection{General procedure for the $\mathrm{Cp} * \mathrm{Co}$ (III)-catalyzed synthesis of 3 and 5}

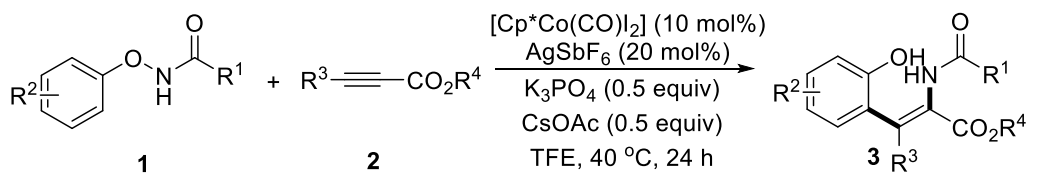

A $10 \mathrm{~mL}$ oven-dried Schlenk tube equipped with a magnetic stir bar was dried under vacuum. The aryloxyacetamide $1\left(0.20 \mathrm{mmol}, 1.0\right.$ equiv) and the $\left[\mathrm{Cp} * \mathrm{Co}(\mathrm{CO}) \mathrm{I}_{2}\right]$-catalyst $(9.5 \mathrm{mg}, 10$ mol\%) were added to the tube. Subsequently, the reaction tube was charged with $\mathrm{AgSbF}_{6}(13.7$ $\mathrm{mg}, 20 \mathrm{~mol} \%), \mathrm{K}_{3} \mathrm{PO}_{4}(21.2 \mathrm{mg}, 50 \mathrm{~mol} \%)$ and $\mathrm{CsOAc}(19.2 \mathrm{mg}, 50 \mathrm{~mol} \%)$, in the glovebox. Dry trifluoroethanol $(1.0 \mathrm{~mL}, 0.2 \mathrm{M})$ was added via syringe, followed by the propiolates $2(0.22$ mmol, 1.1 equiv) via syringe. The Schlenk tube was sealed and stirred for $24 \mathrm{~h}$ at $40{ }^{\circ} \mathrm{C}$ in an oil bath. The crude reaction mixture was diluted with dichloromethane $(5 \mathrm{~mL})$ transferred to a 
$100 \mathrm{~mL}$ round bottom flask, adsorbed on silica and purified by silica gel flash chromatography (PE/ EA).

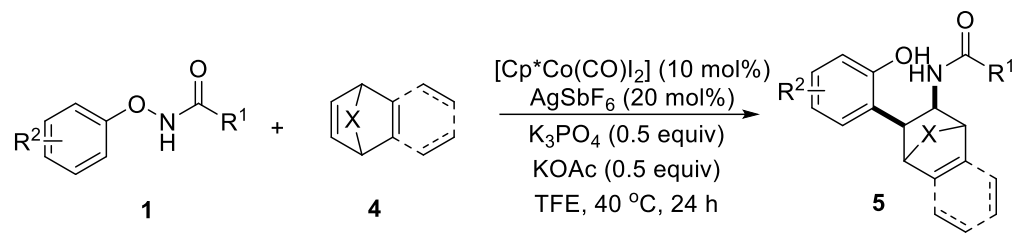

A $10 \mathrm{~mL}$ oven-dried Schlenk tube equipped with a magnetic stir bar was dried under vacuum. The aryloxyacetamide 1 ( $0.20 \mathrm{mmol}, 1.0$ equiv) and the [Cp*Co(CO) $\left.\mathrm{I}_{2}\right]$-catalyst $(9.5 \mathrm{mg}, 10$ mol\%) were added to the tube. Subsequently, the reaction tube was charged with $\mathrm{AgSbF}_{6}(13.7$ $\mathrm{mg}, 20 \mathrm{~mol} \%), \mathrm{K}_{3} \mathrm{PO}_{4}(21.2 \mathrm{mg}, 50 \mathrm{~mol} \%)$ and KOAc $(9.8 \mathrm{mg}, 50 \mathrm{~mol} \%)$, in the glovebox. Dry trifluoroethanol $(1.0 \mathrm{~mL}, 0.2 \mathrm{M})$ was added via syringe, followed by the alkenes $4(0.22$ mmol, 1.1 equiv) via syringe. The Schlenk tube was sealed and stirred for $24 \mathrm{~h}$ at $40{ }^{\circ} \mathrm{C}$ in an oil bath. The crude reaction mixture was diluted with dichloromethane $(5 \mathrm{~mL})$ transferred to a $100 \mathrm{~mL}$ round bottom flask, adsorbed on silica and purified by silica gel flash chromatography (PE/ EA).

\subsection{Gram-Scale Synthesis of 3aa}

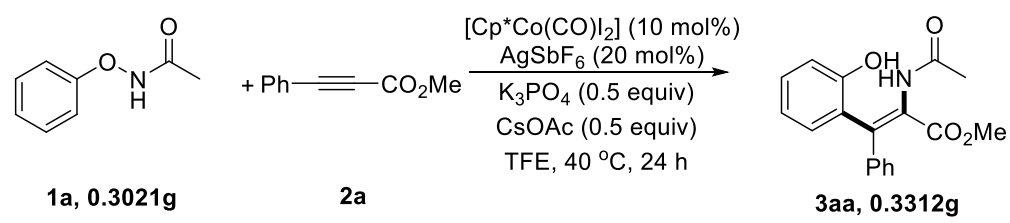

A $50 \mathrm{~mL}$ oven-dried Schlenk tube equipped with a magnetic stir bar was dried under vacuum. $N$-Phenoxyacetamide 1a $(0.3021 \mathrm{~g}, 2.0 \mathrm{mmol})$ and the $\left[\mathrm{Cp} * \mathrm{Co}(\mathrm{CO}) \mathrm{I}_{2}\right]$-catalyst $(95.4 \mathrm{mg}, 0.2$ mmol) were added to the tube. Subsequently, the reaction tube was charged with $\mathrm{AgSbF}_{6}(136.7$ $\mathrm{mg}, 0.4 \mathrm{mmol}), \mathrm{K}_{3} \mathrm{PO}_{4}(211.8 \mathrm{mg}, 1 \mathrm{mmol})$ and CsOAc (0.1920 g, $\left.1 \mathrm{mmol}\right)$, in the glovebox. Dry trifluoroethanol ( $10.0 \mathrm{~mL}, 0.2 \mathrm{M})$ was added via syringe, followed by 3-phenylpropiolate 2a $(0.3521 \mathrm{~g}, 2.2 \mathrm{mmol})$ via syringe. The Schlenk tube was sealed and stirred for $24 \mathrm{~h}$ at $40{ }^{\circ} \mathrm{C}$ in an oil bath. After cooled to room temperature, the reaction mixture was filtered through a pad of Celite. The filtrate was concentrated in vacuo to afford crude product, which was purified by flash column chromatography on silica gel using hexanes/EtOAc (1/1) as the eluent to give the pure product 3aa $(0.3312 \mathrm{~g}, 53 \%)$.

\subsection{Data of products 3 and 5}




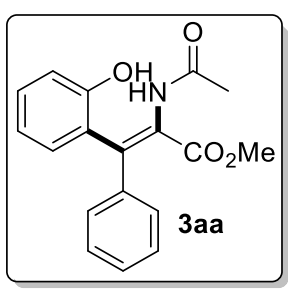

\section{Methyl (Z)-2-acetamido-3-(2-hydroxyphenyl)-3-phenylacrylate (3aa)}

This compound was obtained in $86 \%$ yield $(53.5 \mathrm{mg})$ as white solid. Mp: $201-202{ }^{\circ} \mathrm{C}$. Eluent: $\mathrm{PE} / \mathrm{EA}=1 / 1, \mathrm{R}_{\mathrm{f}}=0.17$.

${ }^{1} \mathrm{H}$ NMR (400 MHz, DMSO- $\left.d_{6}\right) \delta 9.36(\mathrm{~s}, 1 \mathrm{H}), 9.15(\mathrm{~s}, 1 \mathrm{H}), 7.27-7.18(\mathrm{~m}, 3 \mathrm{H}), 7.15$ (ddd, $J$ $=8.5,7.3,1.8 \mathrm{~Hz}, 1 \mathrm{H}), 7.02-6.95(\mathrm{~m}, 2 \mathrm{H}), 6.90(\mathrm{dd}, J=7.6,1.8 \mathrm{~Hz}, 1 \mathrm{H}), 6.86-6.75(\mathrm{~m}, 2 \mathrm{H})$, $3.36(\mathrm{~s}, 3 \mathrm{H}), 1.82(\mathrm{~s}, 3 \mathrm{H})$.

${ }^{13} \mathrm{C}$ NMR (101 MHz, DMSO) $\delta 169.16,166.54,155.51,140.60,133.67,131.50,129.79,128.69$, $128.12,127.53,127.12,126.03,119.25,116.58,51.62,22.47$.

HRMS (ESI) (m/z): calcd for $\mathrm{C}_{18} \mathrm{H}_{17} \mathrm{NO}_{4}[\mathrm{M}+\mathrm{Na}]^{+}: 334.1055$, found: 334.1053 .

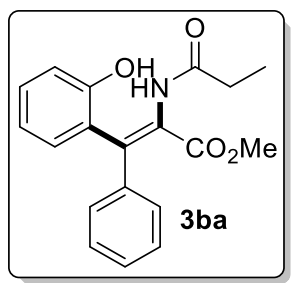

\section{Methyl (Z)-3-(2-hydroxyphenyl)-3-phenyl-2-propionamidoacrylate (3ba)}

This compound was obtained in $68 \%$ yield $(44.2 \mathrm{mg})$ as white solid. Mp: $198-199{ }^{\circ} \mathrm{C}$. Eluent: $\mathrm{PE} / \mathrm{EA}=1 / 1, \mathrm{R}_{\mathrm{f}}=0.65$.

${ }^{1} \mathrm{H}$ NMR $(400 \mathrm{MHz}$, DMSO-d $) \delta 9.35(\mathrm{~s}, 1 \mathrm{H}), 9.04(\mathrm{~s}, 1 \mathrm{H}), 7.28-7.21(\mathrm{~m}, 3 \mathrm{H}), 7.14$ (ddd, $J$ $=8.4,7.3,1.8 \mathrm{~Hz}, 1 \mathrm{H}), 7.00-6.95(\mathrm{~m}, 2 \mathrm{H}), 6.90(\mathrm{dd}, J=7.6,1.8 \mathrm{~Hz}, 1 \mathrm{H}), 6.82(\mathrm{dd}, J=8.1$, $1.1 \mathrm{~Hz}, 1 \mathrm{H}), 6.78(\mathrm{td}, J=7.4,1.2 \mathrm{~Hz}, 1 \mathrm{H}), 3.36(\mathrm{~s}, 3 \mathrm{H}), 2.09$ (q, $J=7.6 \mathrm{~Hz}, 2 \mathrm{H}), 0.94(\mathrm{t}, J=$ $7.6 \mathrm{~Hz}, 3 \mathrm{H})$.

${ }^{13} \mathrm{C}$ NMR (101 MHz, DMSO) $\delta 172.81,166.59,155.50,140.62,133.66,131.55,129.79,128.68$, 128.12, 127.52, 127.09, 126.00, 119.18, 116.55, 51.61, 28.17, 9.97.

HRMS (ESI) (m/z): calcd for $\mathrm{C}_{19} \mathrm{H}_{19} \mathrm{NO}_{4}[\mathrm{M}+\mathrm{Na}]^{+}: 348.1212$, found: 348.1214 .

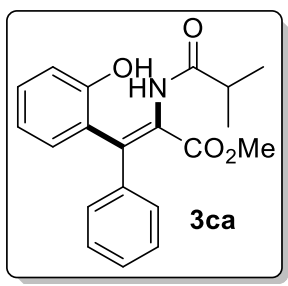

\section{Methyl (Z)-3-(2-hydroxyphenyl)-2-isobutyramido-3-phenylacrylate (3ca)}

This compound was obtained in $51 \%$ yield $(34.6 \mathrm{mg})$ as white solid. $\mathrm{Mp}: 161-162{ }^{\circ} \mathrm{C}$. Eluent: $\mathrm{PE} / \mathrm{EA}=1 / 1, \mathrm{R}_{\mathrm{f}}=0.54$.

${ }^{1} \mathrm{H}$ NMR (400 MHz, DMSO-d $\left.d_{6}\right) \delta 9.33(\mathrm{~s}, 1 \mathrm{H}), 9.00(\mathrm{~s}, 1 \mathrm{H}), 7.29-7.20(\mathrm{~m}, 3 \mathrm{H}), 7.17-7.12$ (m, 1H), $7.00-6.95(\mathrm{~m}, 2 \mathrm{H}), 6.90(\mathrm{dd}, J=7.6,1.8 \mathrm{~Hz}, 1 \mathrm{H}), 6.85-6.80(\mathrm{~m}, 1 \mathrm{H}), 6.78(\mathrm{td}, J=$ 
7.4, $1.1 \mathrm{~Hz}, 1 \mathrm{H}), 3.36(\mathrm{~s}, 3 \mathrm{H}), 2.44(\mathrm{q}, J=6.9 \mathrm{~Hz}, 1 \mathrm{H}), 0.93(\mathrm{~d}, J=6.8 \mathrm{~Hz}, 6 \mathrm{H})$.

${ }^{13} \mathrm{C}$ NMR (101 MHz, DMSO) $\delta 175.28,166.08,154.99,140.09,133.73,131.06,129.32,128.14$, 127.61, 127.03, 126.53, 125.51, 118.59, 116.01, 112.70, 51.09, 32.68, 18.98 .

HRMS (ESI) (m/z): calcd for $\mathrm{C}_{20} \mathrm{H}_{21} \mathrm{NO}_{4}[\mathrm{M}+\mathrm{Na}]^{+}: 362.1368$, found: 362.1365 .

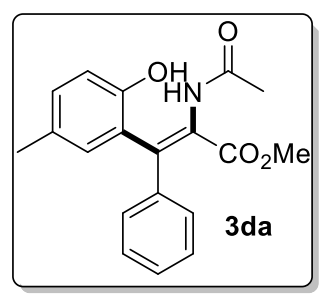

\section{Methyl (Z)-2-acetamido-3-(2-hydroxyphenyl)-3-(p-tolyl)acrylate (3da)}

This compound was obtained in $83 \%$ yield $(54.0 \mathrm{mg})$ as white solid. Mp: $86-87{ }^{\circ} \mathrm{C}$. Eluent: $\mathrm{PE} / \mathrm{EA}=1 / 1, \mathrm{R}_{\mathrm{f}}=0.25$.

${ }^{1} \mathrm{H}$ NMR (400 MHz, DMSO- $\left.d_{6}\right) \delta 9.35(\mathrm{~s}, 1 \mathrm{H}), 9.07(\mathrm{~s}, 1 \mathrm{H}), 7.23(\mathrm{qd}, J=7.7,6.7,3.6 \mathrm{~Hz}, 3 \mathrm{H})$, $7.00-6.88(\mathrm{~m}, 3 \mathrm{H}), 6.84(\mathrm{~d}, J=8.2 \mathrm{~Hz}, 1 \mathrm{H}), 6.65(\mathrm{~d}, J=2.1 \mathrm{~Hz}, 1 \mathrm{H}), 3.34(\mathrm{~s}, 3 \mathrm{H}), 2.13(\mathrm{~s}$, $3 \mathrm{H}), 1.81(\mathrm{~s}, 3 \mathrm{H})$.

${ }^{13} \mathrm{C}$ NMR (101 MHz, DMSO) $\delta$ 169.18, 166.54, 153.34, 140.68, 133.57, 131.55, 130.21, 128.71, $128.12,127.51,127.28,126.99,125.59,116.58,51.60,22.49,20.55$.

HRMS (ESI) $(\mathrm{m} / \mathrm{z})$ : calcd for $\mathrm{C}_{19} \mathrm{H}_{19} \mathrm{NO}_{4}[\mathrm{M}+\mathrm{Na}]^{+}: 348.1212$, found: 348.1211 .

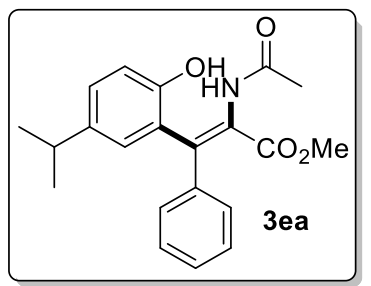

Methyl (Z)-2-acetamido-3-(2-hydroxyphenyl)-3-(4-isopropylphenyl)acrylate (3ea)

This compound was obtained in $76 \%$ yield $(53.7 \mathrm{mg})$ as white solid. $\mathrm{Mp}: 104-105{ }^{\circ} \mathrm{C}$. Eluent: $\mathrm{PE} / \mathrm{EA}=1 / 1, \mathrm{R}_{\mathrm{f}}=0.29$.

${ }^{1} \mathrm{H}$ NMR $\left(400 \mathrm{MHz}\right.$, DMSO- $\left.d_{6}\right) \delta 9.16(\mathrm{~s}, 1 \mathrm{H}), 9.11(\mathrm{~s}, 1 \mathrm{H}), 7.28-7.18(\mathrm{~m}, 3 \mathrm{H}), 7.02(\mathrm{dd}, J=$ 8.3, $2.4 \mathrm{~Hz}, 1 \mathrm{H}), 7.00-6.90(\mathrm{~m}, 2 \mathrm{H}), 6.80(\mathrm{~d}, J=2.3 \mathrm{~Hz}, 1 \mathrm{H}), 6.73(\mathrm{~d}, J=8.3 \mathrm{~Hz}, 1 \mathrm{H}), 3.36$ (s, 3H), $2.76(\mathrm{p}, J=6.9 \mathrm{~Hz}, 1 \mathrm{H}), 1.81(\mathrm{~s}, 3 \mathrm{H}), 1.13(\mathrm{~d}, J=6.9 \mathrm{~Hz}, 6 \mathrm{H})$.

${ }^{13} \mathrm{C}$ NMR (101 MHz, DMSO) $\delta 168.74,166.16,153.16,140.05,138.15,134.54,128.62,128.13$, $127.56,127.25,127.03,126.33,125.05,115.92,51.10,32.34,24.08,21.88$.

HRMS (ESI) (m/z): calcd for $\mathrm{C}_{21} \mathrm{H}_{23} \mathrm{NO}_{4}[\mathrm{M}+\mathrm{Na}]^{+}: 376.1525$, found: 376.1526 .

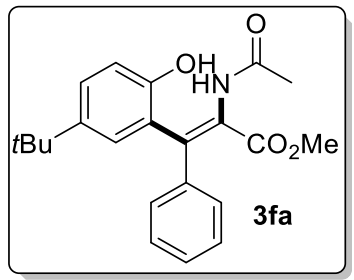

Methyl (Z)-2-acetamido-3-(4-(tert-butyl)phenyl)-3-(2-hydroxyphenyl)acrylate (3fa) 
This compound was obtained in $75 \%$ yield $(55.1 \mathrm{mg})$ as white solid. Mp: $91-92{ }^{\circ} \mathrm{C}$. Eluent: $\mathrm{PE} / \mathrm{EA}=1 / 1, \mathrm{R}_{\mathrm{f}}=0.33$.

${ }^{1} \mathrm{H}$ NMR $\left(400 \mathrm{MHz}\right.$, DMSO- $\left.d_{6}\right) \delta 9.20(\mathrm{~s}, 1 \mathrm{H}), 9.10(\mathrm{~s}, 1 \mathrm{H}), 7.31-7.20(\mathrm{~m}, 3 \mathrm{H}), 7.17(\mathrm{dd}, J=$ 8.5, 2.6 Hz, 1H), $7.04-6.88(\mathrm{~m}, 3 \mathrm{H}), 6.73(\mathrm{~d}, J=8.5 \mathrm{~Hz}, 1 \mathrm{H}), 3.36(\mathrm{~s}, 3 \mathrm{H}), 1.80(\mathrm{~s}, 3 \mathrm{H}), 1.21$ ( $\mathrm{s}, 9 \mathrm{H})$.

${ }^{13} \mathrm{C}$ NMR (101 MHz, DMSO) $\delta 169.31,166.72,153.44,140.92,140.50,135.99,128.57,128.23$, 128.03, 127.54, 126.68, 125.14, 116.16, 51.61, 34.00, 31.80, 22.33.

HRMS (ESI) (m/z): calcd for $\mathrm{C}_{22} \mathrm{H}_{25} \mathrm{NO}_{4}[\mathrm{M}+\mathrm{Na}]^{+}: 390.1681$, found: 390.1682 .

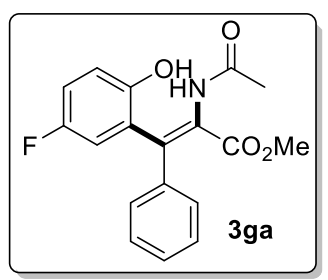

\section{Methyl (Z)-2-acetamido-3-(4-fluorophenyl)-3-(2-hydroxyphenyl)acrylate (3ga)}

This compound was obtained in $71 \%$ yield $(46.7 \mathrm{mg})$ as white solid. $\mathrm{Mp}: 182-184{ }^{\circ} \mathrm{C}$. Eluent: $\mathrm{PE} / \mathrm{EA}=1 / 1, \mathrm{R}_{\mathrm{f}}=0.26$.

${ }^{1} \mathrm{H}$ NMR (400 MHz, DMSO- $\left.d_{6}\right) \delta 9.71(\mathrm{~s}, 1 \mathrm{H}), 9.25(\mathrm{~s}, 1 \mathrm{H}), 7.37-7.16(\mathrm{~m}, 3 \mathrm{H}), 6.98(\mathrm{ddd}, J$ $=6.4,5.1,1.8 \mathrm{~Hz}, 4 \mathrm{H}), 6.62(\mathrm{dt}, J=9.3,1.8 \mathrm{~Hz}, 1 \mathrm{H}), 3.35(\mathrm{~s}, 3 \mathrm{H}), 1.83(\mathrm{~s}, 3 \mathrm{H})$.

${ }^{13} \mathrm{C}$ NMR (101 MHz, DMSO) $\delta 169.22,166.30,156.51,154.18,152.12,140.00,131.98,128.70$, 128.24, 127.71, 126.76, 126.69, 117.57, 117.49, 117.19, 116.96, 116.15, 115.92, 51.70, 22.48. ${ }^{19} \mathrm{~F}$ NMR (376 MHz, DMSO) $\delta-126.34$.

HRMS (ESI) (m/z): calcd for $\mathrm{C}_{18} \mathrm{H}_{16} \mathrm{FNO}_{4}[\mathrm{M}+\mathrm{Na}]^{+}$: 352.0961, found: 352.0960 .

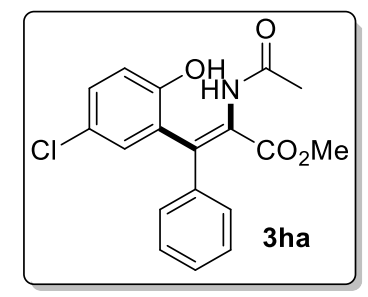

Methyl (Z)-2-acetamido-3-(4-chlorophenyl)-3-(2-hydroxyphenyl)acrylate (3ha)

This compound was obtained in $68 \%$ yield $(46.9 \mathrm{mg})$ as white solid. $\mathrm{Mp}: 168-169{ }^{\circ} \mathrm{C}$. Eluent: $\mathrm{PE} / \mathrm{EA}=1 / 1, \mathrm{R}_{\mathrm{f}}=0.35$.

${ }^{1} \mathrm{H}$ NMR (400 MHz, DMSO- $\left.d_{6}\right) \delta 9.76(\mathrm{~s}, 1 \mathrm{H}), 9.28(\mathrm{~s}, 1 \mathrm{H}), 7.30-7.23(\mathrm{~m}, 3 \mathrm{H}), 7.19(\mathrm{dd}, J=$ 8.7, $2.7 \mathrm{~Hz}, 1 \mathrm{H}), 7.02-6.97(\mathrm{~m}, 2 \mathrm{H}), 6.91-6.77(\mathrm{~m}, 2 \mathrm{H}), 3.37$ (s, 3H), 1.84 (s, 3H).

${ }^{13} \mathrm{C}$ NMR (101 MHz, DMSO) $\delta 168.69,165.74,154.14,139.50,130.96,130.15,128.83,128.25$, $127.82,127.41,127.29,127.25,121.88,117.78,51.21,21.97$.

HRMS (ESI) (m/z): calcd for $\mathrm{C}_{18} \mathrm{H}_{16} \mathrm{ClNO}_{4}[\mathrm{M}+\mathrm{Na}]^{+}: 368.0666$, found: 368.0664 . 


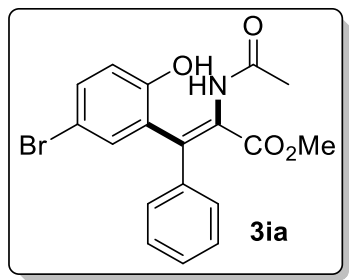

Methyl (Z)-2-acetamido-3-(4-bromophenyl)-3-(2-hydroxyphenyl)acrylate (3ia)

This compound was obtained in $61 \%$ yield $(47.5 \mathrm{mg})$ as white solid. $\mathrm{Mp}: 136-138^{\circ} \mathrm{C}$. Eluent: $\mathrm{PE} / \mathrm{EA}=1 / 1, \mathrm{R}_{\mathrm{f}}=0.26$.

${ }^{1} \mathrm{H}$ NMR (400 MHz, DMSO-d $\left.d_{6}\right) \delta 9.78(\mathrm{~d}, J=1.9 \mathrm{~Hz}, 1 \mathrm{H}), 9.27$ (s, $\left.1 \mathrm{H}\right), 7.28$ (ddd, $J=19.1$, 8.6, $5.5 \mathrm{~Hz}, 4 \mathrm{H}), 6.98$ (dd, $J=6.5,1.8 \mathrm{~Hz}, 2 \mathrm{H}), 6.93$ (d, $J=2.5 \mathrm{~Hz}, 1 \mathrm{H}), 6.81$ (d, $J=8.8 \mathrm{~Hz}$, $1 \mathrm{H}), 3.36(\mathrm{~s}, 3 \mathrm{H}), 1.83(\mathrm{~s}, 3 \mathrm{H})$.

${ }^{13} \mathrm{C}$ NMR (101 MHz, DMSO) $\delta 169.16,166.22,154.95,140.00,133.56,132.24,131.28,128.76$, $128.35,127.95,127.82,127.48,118.74,110.05,51.72,22.47$.

HRMS (ESI) (m/z): calcd for $\mathrm{C}_{18} \mathrm{H}_{16} \mathrm{BrNO}_{4}[\mathrm{M}+\mathrm{Na}]^{+}: 412.0160$, found: 412.0157 .

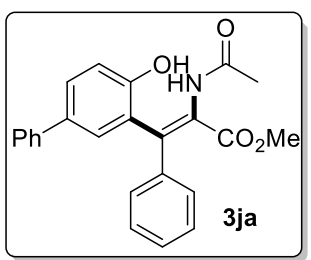

Methyl (Z)-3-([1,1'-biphenyl]-4-yl)-2-acetamido-3-(2-hydroxyphenyl)acrylate (3ja)

This compound was obtained in $80 \%$ yield $(61.9 \mathrm{mg})$ as white solid. Mp: $75-76{ }^{\circ} \mathrm{C}$. Eluent: $\mathrm{PE} / \mathrm{EA}=1 / 1, \mathrm{R}_{\mathrm{f}}=0.29$.

${ }^{1} \mathrm{H}$ NMR $\left(400 \mathrm{MHz}, \mathrm{DMSO}-d_{6}\right) \delta 9.68(\mathrm{~s}, 1 \mathrm{H}), 9.29(\mathrm{~s}, 1 \mathrm{H}), 7.48(\mathrm{td}, J=5.6,2.4 \mathrm{~Hz}, 3 \mathrm{H}), 7.41$ $(\mathrm{d}, J=7.5 \mathrm{~Hz}, 2 \mathrm{H}), 7.26$ (qd, $J=8.5,7.6,4.1 \mathrm{~Hz}, 4 \mathrm{H}), 7.16(\mathrm{~d}, J=2.4 \mathrm{~Hz}, 1 \mathrm{H}), 7.08-7.01(\mathrm{~m}$, 2H), $6.97(\mathrm{~d}, J=8.4 \mathrm{~Hz}, 1 \mathrm{H}), 3.38(\mathrm{~s}, 3 \mathrm{H}), 1.84(\mathrm{~s}, 3 \mathrm{H})$.

${ }^{13} \mathrm{C}$ NMR (101 MHz, DMSO) $\delta 169.25,166.51,155.40,140.48,140.39,133.48,131.20,129.63$, 129.34, 128.78, 128.20, 128.17, 127.64, 127.41, 126.95, 126.45, 126.37, 117.19, 51.67, 22.49. HRMS (ESI) (m/z): calcd for $\mathrm{C}_{24} \mathrm{H}_{21} \mathrm{NO}_{4}[\mathrm{M}+\mathrm{Na}]^{+}: 410.1368$, found: 410.1367 .

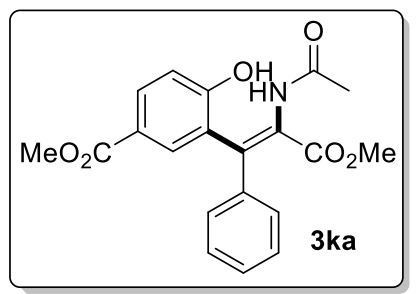

\section{Methyl}

(Z)-3-(2-acetamido-3-methoxy-3-oxo-1-phenylprop-1-en-1-yl)-4hydroxybenzoate (3ka)

This compound was obtained in $56 \%$ yield $(41.3 \mathrm{mg})$ as white solid. Mp: $177-179{ }^{\circ} \mathrm{C}$. Eluent: $\mathrm{PE} / \mathrm{EA}=1 / 1, \mathrm{R}_{\mathrm{f}}=0.20$.

${ }^{1} \mathrm{H}$ NMR (400 MHz, DMSO- $\left.d_{6}\right) \delta 10.42(\mathrm{~s}, 1 \mathrm{H}), 9.26(\mathrm{~s}, 1 \mathrm{H}), 7.79(\mathrm{dd}, J=8.5,2.3 \mathrm{~Hz}, 1 \mathrm{H})$, $7.51(\mathrm{~d}, J=2.3 \mathrm{~Hz}, 1 \mathrm{H}), 7.27(\mathrm{q}, J=7.7,6.8 \mathrm{~Hz}, 3 \mathrm{H}), 7.08-6.86(\mathrm{~m}, 3 \mathrm{H}), 3.76(\mathrm{~s}, 3 \mathrm{H}), 3.38$ (s, 3H), $1.81(\mathrm{~s}, 3 \mathrm{H})$.

${ }^{13} \mathrm{C}$ NMR (101 MHz, DMSO) $\delta$ 168.62, 165.86, 165.80, 159.61, 139.56, 132.96, 131.18, 130.88, 
$128.20,127.88,127.47,127.32,125.74,120.11,116.15,51.68,51.24,21.94$.

HRMS (ESI) (m/z): calcd for $\mathrm{C}_{20} \mathrm{H}_{19} \mathrm{NO}_{6}[\mathrm{M}+\mathrm{Na}]^{+}: 392.1110$, found: 392.1112 .<smiles>CC(=O)NC(=O)c1c(O)cccc1-c1ccccc1</smiles>

Methyl (Z)-2-acetamido-3-(2-hydroxy-5-nitrophenyl)-3-phenylacrylate (3la)

This compound was obtained in $43 \%$ yield $\left(30.6 \mathrm{mg}\right.$ ) as yellow solid. Mp: $105-106^{\circ} \mathrm{C}$. Eluent: $\mathrm{PE} / \mathrm{EA}=1 / 1, \mathrm{R}_{\mathrm{f}}=0.22$.

${ }^{1} \mathrm{H}$ NMR (400 MHz, DMSO- $d_{6}$ ) $\delta 11.19(\mathrm{~s}, 1 \mathrm{H}), 9.38(\mathrm{~s}, 1 \mathrm{H}), 8.12$ (dd, $\left.J=9.0,2.9 \mathrm{~Hz}, 1 \mathrm{H}\right)$, $7.75(\mathrm{~d}, J=2.9 \mathrm{~Hz}, 1 \mathrm{H}), 7.29$ (d, $J=7.2 \mathrm{~Hz}, 3 \mathrm{H}), 7.10-6.93$ (m, 3H), 3.39 (s, 3H), 1.83 (s, $3 \mathrm{H})$.

${ }^{13} \mathrm{C}$ NMR (101 MHz, DMSO) $\delta 168.67,165.54,161.72,139.27,138.91,129.68,128.25,128.20$, $128.01,127.55,127.33,126.31,125.57,116.52,51.35,21.95$.

HRMS (ESI) (m/z): calcd for $\mathrm{C}_{18} \mathrm{H}_{16} \mathrm{~N}_{2} \mathrm{O}_{6}[\mathrm{M}+\mathrm{Na}]^{+}: 379.0906$, found: 379.0905 .

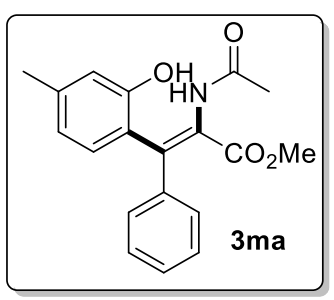

Methyl (Z)-2-acetamido-3-(2-hydroxy-4-methylphenyl)-3-phenylacrylate (3ma)

This compound was obtained in $73 \%$ yield $(47.5 \mathrm{mg})$ as white solid. Mp: $199-201{ }^{\circ} \mathrm{C}$. Eluent: $\mathrm{PE} / \mathrm{EA}=1 / 1, \mathrm{R}_{\mathrm{f}}=0.26$.

${ }^{1} \mathrm{H}$ NMR (400 MHz, DMSO-d $\left.)_{6}\right) \delta 9.26(\mathrm{~s}, 1 \mathrm{H}), 9.10$ (s, 1H), 7.24 (dddd, $J=9.0,5.3,3.7,1.5$ $\mathrm{Hz}, 3 \mathrm{H}), 7.00-6.94(\mathrm{~m}, 2 \mathrm{H}), 6.77(\mathrm{~d}, J=7.7 \mathrm{~Hz}, 1 \mathrm{H}), 6.65-6.58(\mathrm{~m}, 2 \mathrm{H}), 3.35(\mathrm{~s}, 3 \mathrm{H}), 2.23$ (s, 3H), $1.82(\mathrm{~s}, 3 \mathrm{H})$.

${ }^{13} \mathrm{C}$ NMR (101 MHz, DMSO) $\delta$ 169.12, 166.60, 155.39, 140.80, 139.29, 133.63, 131.42, 128.72 , 128.09, 127.49, 126.89, 123.20, 120.14, 117.11, 51.59, 22.49, 21.38.

HRMS (ESI) (m/z): calcd for $\mathrm{C}_{19} \mathrm{H}_{19} \mathrm{NO}_{4}[\mathrm{M}+\mathrm{Na}]^{+}: 348.1212$, found: 348.1210 .

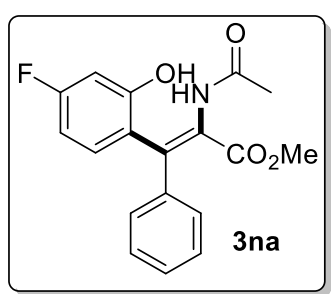

Methyl (Z)-2-acetamido-3-(4-fluoro-2-hydroxyphenyl)-3-phenylacrylate (3na)

This compound was obtained in $61 \%$ yield $(40.2 \mathrm{mg})$ as white solid. Mp: $161-163{ }^{\circ} \mathrm{C}$. Eluent: $\mathrm{PE} / \mathrm{EA}=1 / 1, \mathrm{R}_{\mathrm{f}}=0.27$.

${ }^{1} \mathrm{H}$ NMR (400 MHz, DMSO-d $) \delta 9.82(\mathrm{~d}, J=1.7 \mathrm{~Hz}, 1 \mathrm{H}), 9.28(\mathrm{~s}, 1 \mathrm{H}), 7.27-7.16(\mathrm{~m}, 4 \mathrm{H})$, 
$7.03-6.96(\mathrm{~m}, 2 \mathrm{H}), 6.68(\mathrm{~d}, J=8.2 \mathrm{~Hz}, 1 \mathrm{H}), 6.61(\mathrm{dd}, J=9.1,3.1 \mathrm{~Hz}, 1 \mathrm{H}), 3.34(\mathrm{~s}, 3 \mathrm{H}), 1.83$ $(\mathrm{s}, 3 \mathrm{H})$.

${ }^{13} \mathrm{C}$ NMR (101 MHz, DMSO) $\delta 168.89,166.09,162.02,159.59,157.14,157.07,139.70,130.39$, $130.28,129.37,128.66,128.20,128.18,127.54,125.28,114.34,114.16,112.15,112.12,106.28$, $106.05,51.72,22.47$.

${ }^{19} \mathrm{~F}$ NMR (376 MHz, DMSO) $\delta-112.67$.

HRMS (ESI) (m/z): calcd for $\mathrm{C}_{18} \mathrm{H}_{16} \mathrm{FNO}_{4}[\mathrm{M}+\mathrm{Na}]^{+}: 352.0961$, found: 352.0962 .

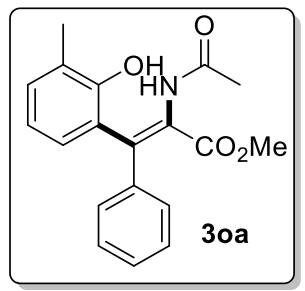

\section{Methyl (Z)-2-acetamido-3-(2-hydroxy-3-methylphenyl)-3-phenylacrylate (3oa)}

This compound was obtained in $54 \%$ yield $(35.1 \mathrm{mg})$ as white solid. Mp: $93-94{ }^{\circ} \mathrm{C}$. Eluent: $\mathrm{PE} / \mathrm{EA}=1 / 1, \mathrm{R}_{\mathrm{f}}=0.43$.

${ }^{1} \mathrm{H}$ NMR (400 MHz, DMSO-d $\left.)\right) \delta 9.15(\mathrm{~s}, 1 \mathrm{H}), 8.22(\mathrm{~s}, 1 \mathrm{H}), 7.28-7.19(\mathrm{~m}, 3 \mathrm{H}), 7.10-7.04$ (m, 1H), $7.04-6.98(\mathrm{~m}, 2 \mathrm{H}), 6.84-6.73(\mathrm{~m}, 2 \mathrm{H}), 3.37$ (s, 3H), $2.14(\mathrm{~s}, 3 \mathrm{H}), 1.82(\mathrm{~s}, 3 \mathrm{H})$.

${ }^{13} \mathrm{C}$ NMR (101 MHz, DMSO) $\delta 168.77,166.04,152.83,139.96,133.81,130.77,128.40,128.17$, 127.57, 127.04, 126.70, 126.47, 125.13, 119.21, 51.12, 21.94, 16.71.

HRMS (ESI) (m/z): calcd for $\mathrm{C}_{19} \mathrm{H}_{19} \mathrm{NO}_{4}[\mathrm{M}+\mathrm{Na}]^{+}: 348.1212$, found: 348.1211 .<smiles>CC(=O)NC(C(C)=O)=C(c1ccccc1)c1cccc(Cl)c1O</smiles>

\section{Methyl (Z)-2-acetamido-3-(3-chloro-2-hydroxyphenyl)-3-phenylacrylate (3pa)}

This compound was obtained in $45 \%$ yield $(31.1 \mathrm{mg})$ as white solid. $\mathrm{Mp}: 102-104{ }^{\circ} \mathrm{C}$. Eluent: $\mathrm{PE} / \mathrm{EA}=1 / 1, \mathrm{R}_{\mathrm{f}}=0.37$.

${ }^{1} \mathrm{H}$ NMR (400 MHz, DMSO- $\left.d_{6}\right) \delta 9.30(\mathrm{~s}, 1 \mathrm{H}), 9.18(\mathrm{~s}, 1 \mathrm{H}), 7.34(\mathrm{dd}, J=7.8,1.8 \mathrm{~Hz}, 1 \mathrm{H}), 7.24$ $(\mathrm{dd}, J=9.3,6.8 \mathrm{~Hz}, 3 \mathrm{H}), 7.04-6.97(\mathrm{~m}, 2 \mathrm{H}), 6.94(\mathrm{dd}, J=7.6,1.8 \mathrm{~Hz}, 1 \mathrm{H}), 6.88(\mathrm{t}, J=7.7$ $\mathrm{Hz}, 1 \mathrm{H}), 3.38$ (s, 3H), $1.82(\mathrm{~s}, 3 \mathrm{H})$.

${ }^{13} \mathrm{C}$ NMR (101 MHz, DMSO) $\delta 168.72,165.81,150.63,139.26,131.67,129.73,129.46,128.53$, $128.14,127.73,127.45,127.25,121.35,120.22,51.23,21.93$.

HRMS (ESI) (m/z): calcd for $\mathrm{C}_{18} \mathrm{H}_{16} \mathrm{ClNO}_{4}[\mathrm{M}+\mathrm{Na}]^{+}: 368.0666$, found: 368.0662 . 


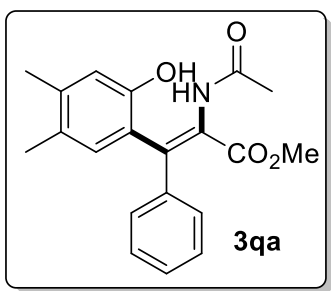

Methyl (Z)-2-acetamido-3-(2-hyd roxy-4,5-dimethylphenyl)-3-phenylacrylate (3qa)

This compound was obtained in $64 \%$ yield $(43.4 \mathrm{mg})$ as white solid. Mp: $87-89{ }^{\circ} \mathrm{C}$. Eluent: $\mathrm{PE} / \mathrm{EA}=1 / 1, \mathrm{R}_{\mathrm{f}}=0.29$.

${ }^{1} \mathrm{H}$ NMR (400 MHz, DMSO-d $\left.d_{6}\right) \quad 9.04(\mathrm{~s}, 1 \mathrm{H}), 9.03(\mathrm{~s}, 1 \mathrm{H}), 7.29-7.18(\mathrm{~m}, 3 \mathrm{H}), 7.04-6.90$ (m, 2H), 6.61 (d, J=12.0 Hz, 2H), 3.34 (s, 3H), $2.13(\mathrm{~s}, 3 \mathrm{H}), 2.04$ (s, 3H), 1.82 (s, 3H).

${ }^{13} \mathrm{C}$ NMR (101 MHz, DMSO) $\delta 168.57,166.09,152.80,140.41,137.21,132.67,131.75,128.30$, $127.61,126.99,126.38,125.88,122.69,117.25,51.06,22.01,19.36,18.37$.

HRMS (ESI) (m/z): calcd for $\mathrm{C}_{20} \mathrm{H}_{21} \mathrm{NO}_{4}[\mathrm{M}+\mathrm{Na}]^{+}: 362.1368$, found: 362.1367 .

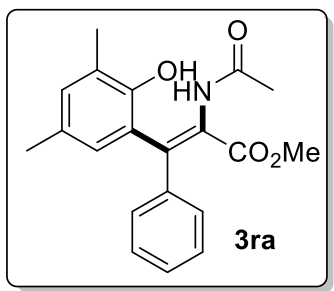

Methyl (Z)-2-acetamido-3-(2-hydroxy-3,5-dimethylphenyl)-3-phenylacrylate (3ra)

This compound was obtained in $52 \%$ yield $(35.3 \mathrm{mg})$ as white solid. Mp: $160-161{ }^{\circ} \mathrm{C}$. Eluent: $\mathrm{PE} / \mathrm{EA}=1 / 1, \mathrm{R}_{\mathrm{f}}=0.46$.

${ }^{1} \mathrm{H}$ NMR (400 MHz, DMSO-d $\left.d_{6}\right) \delta 9.08(\mathrm{~s}, 1 \mathrm{H}), 7.97(\mathrm{~s}, 1 \mathrm{H}), 7.27-7.18(\mathrm{~m}, 3 \mathrm{H}), 7.04-6.96$ $(\mathrm{m}, 2 \mathrm{H}), 6.87(\mathrm{~d}, J=2.3 \mathrm{~Hz}, 1 \mathrm{H}), 6.65-6.49(\mathrm{~m}, 1 \mathrm{H}), 3.36(\mathrm{~s}, 3 \mathrm{H}), 2.15(\mathrm{~s}, 3 \mathrm{H}), 2.11(\mathrm{~s}, 3 \mathrm{H})$, $1.82(\mathrm{~s}, 3 \mathrm{H})$.

${ }^{13} \mathrm{C}$ NMR (101 MHz, DMSO) $\delta 168.77,166.02,150.46,140.06,133.60,131.43,128.54,128.23$, 127.58, 127.48, 127.03, 126.63, 126.30, 125.01, 51.10, 21.96, 20.05, 16.68.

HRMS (ESI) (m/z): calcd for $\mathrm{C}_{20} \mathrm{H}_{21} \mathrm{NO}_{4}[\mathrm{M}+\mathrm{Na}]^{+}: 362.1368$, found: 362.1369 .

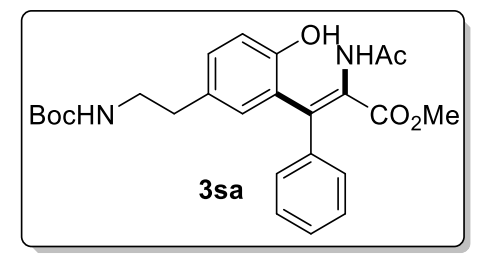

Methyl (Z)-2-acetamido-3-(5-(2-((tert-butoxycarbonyl)amino)ethyl)-2-hydroxyphenyl)3-phenylacrylate (3sa)

This compound was obtained in $70 \%$ yield $(63.6 \mathrm{mg})$ as white solid. Mp: $81-82{ }^{\circ} \mathrm{C}$. Eluent: $\mathrm{PE} / \mathrm{EA}=1 / 1, \mathrm{R}_{\mathrm{f}}=0.15$.

${ }^{1} \mathrm{H}$ NMR (400 MHz, DMSO- $\left.d_{6}\right) \delta 9.19(\mathrm{~s}, 1 \mathrm{H}), 9.08(\mathrm{~s}, 1 \mathrm{H}), 7.23(\mathrm{~d}, J=7.4 \mathrm{~Hz}, 3 \mathrm{H}), 7.02-$ $6.90(\mathrm{~m}, 3 \mathrm{H}), 6.82-6.61(\mathrm{~m}, 3 \mathrm{H}), 3.36(\mathrm{~s}, 3 \mathrm{H}), 3.05(\mathrm{q}, J=6.9 \mathrm{~Hz}, 2 \mathrm{H}), 2.54(\mathrm{t}, J=7.7 \mathrm{~Hz}$, $2 \mathrm{H}), 1.83(\mathrm{~s}, 3 \mathrm{H}), 1.36(\mathrm{~s}, 9 \mathrm{H})$.

${ }^{13} \mathrm{C}$ NMR (101 MHz, DMSO) $\delta 168.63,166.05,155.43,153.42,140.00,133.05,131.06,129.56$, 
$129.27,128.18,127.56,127.01,126.54,125.17,116.03,77.44,51.12,41.97,34.70,28.22$, 21.97.

HRMS (ESI) (m/z): calcd for $\mathrm{C}_{25} \mathrm{H}_{30} \mathrm{~N}_{2} \mathrm{O}_{6}[\mathrm{M}+\mathrm{Na}]^{+}$: 477.2002, found: 477.2001.

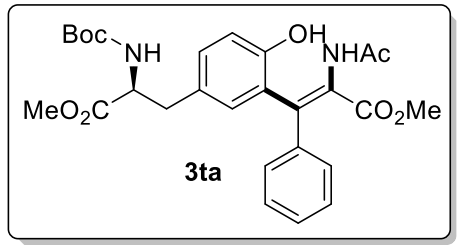

Methyl

(S,Z)-2-acetamido-3-(5-(2-((tert-butoxycarbonyl)amino)-3-methoxy-3oxopropyl)-2-hydroxyphenyl)-3-phenylacrylate (3ta)

This compound was obtained in $72 \%$ yield $(73.7 \mathrm{mg})$ as white solid. Mp: $97-98{ }^{\circ} \mathrm{C}$. Eluent: $\mathrm{PE} / \mathrm{EA}=1 / 1, \mathrm{R}_{\mathrm{f}}=0.17$.

${ }^{1} \mathrm{H}$ NMR (400 MHz, DMSO- $\left.d_{6}\right) \delta 9.22(\mathrm{~s}, 1 \mathrm{H}), 9.08(\mathrm{~s}, 1 \mathrm{H}), 7.33-7.14(\mathrm{~m}, 4 \mathrm{H}), 7.09-6.96$ $(\mathrm{m}, 3 \mathrm{H}), 6.86(\mathrm{~d}, J=2.2 \mathrm{~Hz}, 1 \mathrm{H}), 6.72(\mathrm{~d}, J=8.3 \mathrm{~Hz}, 1 \mathrm{H}), 4.14-4.03(\mathrm{~m}, 1 \mathrm{H}), 3.57(\mathrm{~s}, 3 \mathrm{H})$, $3.37(\mathrm{~s}, 3 \mathrm{H}), 2.92-2.70(\mathrm{~m}, 2 \mathrm{H}), 1.84(\mathrm{~s}, 3 \mathrm{H}), 1.34(\mathrm{~s}, 9 \mathrm{H})$.

${ }^{13} \mathrm{C}$ NMR (101 MHz, DMSO) $\delta 172.58,168.67,166.04,155.40,153.80,139.88,133.00,131.56$, $130.01,128.10,127.50,127.39,126.99,126.57,125.16,115.99,78.22,55.58,51.67,51.13$, 35.63, 28.11, 27.83, 22.00 .

HRMS (ESI) (m/z): calcd for $\mathrm{C}_{27} \mathrm{H}_{32} \mathrm{~N}_{2} \mathrm{O}_{8}[\mathrm{M}+\mathrm{Na}]^{+}$: 535.2056, found: 535.2057.

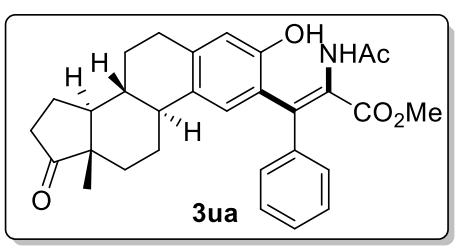

Methyl (Z)-2-acetamido-3-((8R,9S,13S,14S)-3-hydroxy-13-methyl-17-oxo-

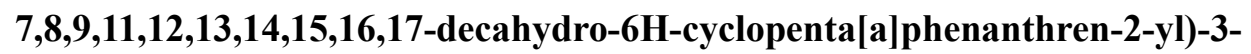
phenylacrylate (3ua)

This compound was obtained in $66 \%$ yield $(64.3 \mathrm{mg})$ as white solid. $\mathrm{Mp}: 141-143{ }^{\circ} \mathrm{C}$. Eluent: $\mathrm{PE} / \mathrm{EA}=1 / 1, \mathrm{R}_{\mathrm{f}}=0.16$.

${ }^{1} \mathrm{H}$ NMR (400 MHz, DMSO- $\left.d_{6}\right) \delta 9.13(\mathrm{~s}, 1 \mathrm{H}), 9.08(\mathrm{~s}, 1 \mathrm{H}), 7.23(\mathrm{dd}, J=9.3,6.9 \mathrm{~Hz}, 3 \mathrm{H}), 7.02$ - $6.93(\mathrm{~m}, 2 \mathrm{H}), 6.78(\mathrm{~s}, 1 \mathrm{H}), 6.54(\mathrm{~s}, 1 \mathrm{H}), 3.35(\mathrm{~s}, 3 \mathrm{H}), 2.84-2.73(\mathrm{~m}, 2 \mathrm{H}), 2.47-2.37(\mathrm{~m}$, $1 \mathrm{H}), 2.10(\mathrm{td}, J=20.3,18.7,7.4 \mathrm{~Hz}, 3 \mathrm{H}), 1.97-1.88(\mathrm{~m}, 2 \mathrm{H}), 1.83(\mathrm{~s}, 3 \mathrm{H}), 1.75-1.67(\mathrm{~m}$, $1 \mathrm{H}), 1.58-1.45(\mathrm{~m}, 3 \mathrm{H}), 1.37-1.25(\mathrm{~m}, 3 \mathrm{H}), 0.81(\mathrm{~s}, 3 \mathrm{H})$.

${ }^{13} \mathrm{C}$ NMR (101 MHz, DMSO) $\delta 220.03,169.20,166.64,153.32,140.85,137.98,134.30,130.21$, $128.73,128.34,128.08,127.51,126.79,123.50,116.29,51.57,49.96,47.73,43.68,38.34$, $35.83,31.70,29.32,26.49,26.01,22.49,21.59,13.96$.

HRMS (ESI) (m/z): calcd for $\mathrm{C}_{30} \mathrm{H}_{33} \mathrm{NO}_{5}[\mathrm{M}+\mathrm{Na}]^{+}:$510.2256, found: 510.2255 . 


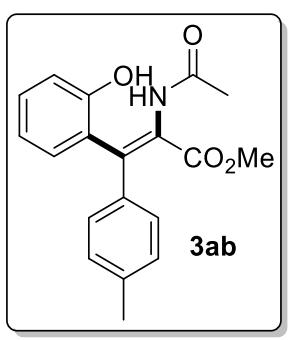

Methyl (Z)-2-acetamido-3-(2-hydroxyphenyl)-3-(p-tolyl)acrylate (3ab)

This compound was obtained in $78 \%$ yield $(50.7 \mathrm{mg})$ as white solid. Mp: $87-89{ }^{\circ} \mathrm{C}$. Eluent: $\mathrm{PE} / \mathrm{EA}=1 / 1, \mathrm{R}_{\mathrm{f}}=0.20$.

${ }^{1} \mathrm{H}$ NMR (400 MHz, DMSO-d $\left.d_{6}\right) \quad 9.33(\mathrm{~s}, 1 \mathrm{H}), 9.11(\mathrm{~s}, 1 \mathrm{H}), 7.18-7.11(\mathrm{~m}, 1 \mathrm{H}), 7.05(\mathrm{~d}, J=$ $7.8 \mathrm{~Hz}, 2 \mathrm{H}), 6.92-6.75(\mathrm{~m}, 5 \mathrm{H}), 3.38(\mathrm{~s}, 3 \mathrm{H}), 2.26(\mathrm{~s}, 3 \mathrm{H}), 1.81(\mathrm{~s}, 3 \mathrm{H})$.

${ }^{13} \mathrm{C} \mathrm{NMR}(101 \mathrm{MHz}, \mathrm{DMSO}) \delta 169.12,166.66,155.52,137.71,136.78,133.69,131.55,129.72$, $128.75,128.65,126.62,126.19,119.19,116.57,51.64,22.47,21.21$.

HRMS (ESI) (m/z): calcd for $\mathrm{C}_{19} \mathrm{H}_{19} \mathrm{NO}_{4}[\mathrm{M}+\mathrm{Na}]^{+}: 348.1212$, found: 348.1211 .<smiles>CCCCc1ccccc1C(C(=O)NC(C)=O)c1ccc(OC)cc1</smiles>

Methyl (Z)-2-acetamido-3-(2-hydroxyphenyl)-3-(4-methoxyphenyl)acrylate (3ac)

This compound was obtained in $60 \%$ yield $(40.9 \mathrm{mg})$ as white solid. Mp: $136-137^{\circ} \mathrm{C}$. Eluent: $\mathrm{PE} / \mathrm{EA}=1 / 1, \mathrm{R}_{\mathrm{f}}=0.17$.

${ }^{1} \mathrm{H}$ NMR $\left(400 \mathrm{MHz}, \mathrm{DMSO}-d_{6}\right) \delta 9.35(\mathrm{~s}, 1 \mathrm{H}), 9.10(\mathrm{~s}, 1 \mathrm{H}), 7.15(\mathrm{t}, J=7.8 \mathrm{~Hz}, 1 \mathrm{H}), 6.90(\mathrm{~d}, J$ $=8.0 \mathrm{~Hz}, 3 \mathrm{H}), 6.87-6.70(\mathrm{~m}, 4 \mathrm{H}), 3.73(\mathrm{~s}, 3 \mathrm{H}), 3.41(\mathrm{~s}, 3 \mathrm{H}), 1.81(\mathrm{~s}, 3 \mathrm{H})$.

${ }^{13} \mathrm{C}$ NMR (101 MHz, DMSO) $\delta 168.61,166.28,158.43,155.02,133.17,132.35,131.04,129.45$, 129.21, 125.77, 125.66, 118.70, 116.07, 113.12, 54.96, 51.13, 21.95.

HRMS (ESI) (m/z): calcd for $\mathrm{C}_{19} \mathrm{H}_{19} \mathrm{NO}_{5}[\mathrm{M}+\mathrm{Na}]^{+}: 364.1161$, found: 364.1159 .

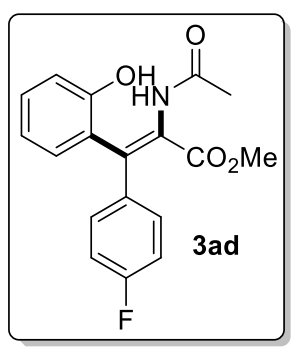

Methyl (Z)-2-acetamido-3-(4-fluorophenyl)-3-(2-hydroxyphenyl)acrylate (3ad)

This compound was obtained in $73 \%$ yield $(48.1 \mathrm{mg})$ as white solid. Mp: $182-184^{\circ} \mathrm{C}$. Eluent: $\mathrm{PE} / \mathrm{EA}=1 / 1, \mathrm{R}_{\mathrm{f}}=0.23$.

${ }^{1} \mathrm{H}$ NMR (400 MHz, DMSO- $\left.d_{6}\right) \delta 9.39(\mathrm{~s}, 1 \mathrm{H}), 9.20(\mathrm{~s}, 1 \mathrm{H}), 7.15$ (ddd, $J=8.1,7.2,1.8 \mathrm{~Hz}$, 1H), $7.12-7.06(\mathrm{~m}, 2 \mathrm{H}), 7.02-6.97(\mathrm{~m}, 2 \mathrm{H}), 6.92(\mathrm{dd}, J=7.6,1.8 \mathrm{~Hz}, 1 \mathrm{H}), 6.84-6.77(\mathrm{~m}$, $2 \mathrm{H}), 3.39(\mathrm{~s}, 3 \mathrm{H}), 1.81(\mathrm{~s}, 3 \mathrm{H})$. 
${ }^{13} \mathrm{C}$ NMR (101 MHz, DMSO) $\delta 169.21,166.43,162.96,160.54,155.54,136.99,136.95,132.75$, 131.38, 130.60, 130.52, 129.94, 127.29, 125.84, 119.32, 116.65, 115.16, 114.95, 51.71, 22.44. ${ }^{19} \mathrm{~F}$ NMR (376 MHz, DMSO) $\delta-115.23$.

HRMS (ESI) (m/z): calcd for $\mathrm{C}_{18} \mathrm{H}_{16} \mathrm{FNO}_{4}[\mathrm{M}+\mathrm{Na}]^{+}: 352.0961$, found: 352.0958 .

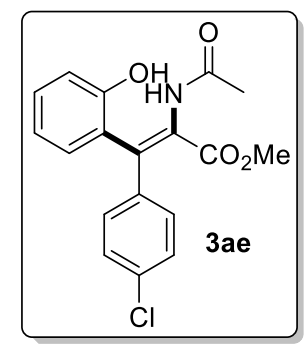

Methyl (Z)-2-acetamido-3-(4-chlorophenyl)-3-(2-hydroxyphenyl)acrylate (3ae)

This compound was obtained in $62 \%$ yield $(42.8 \mathrm{mg})$ as white solid. Mp: $108-109{ }^{\circ} \mathrm{C}$. Eluent: $\mathrm{PE} / \mathrm{EA}=1 / 1, \mathrm{R}_{\mathrm{f}}=0.21$.

${ }^{1} \mathrm{H}$ NMR (400 MHz, DMSO- $\left.d_{6}\right) \delta 9.40(\mathrm{~s}, 1 \mathrm{H}), 9.25(\mathrm{~s}, 1 \mathrm{H}), 7.35-7.29(\mathrm{~m}, 2 \mathrm{H}), 7.16$ (ddd, $J$ $=8.7,7.3,1.8 \mathrm{~Hz}, 1 \mathrm{H}), 6.99-6.90(\mathrm{~m}, 3 \mathrm{H}), 6.85-6.77(\mathrm{~m}, 2 \mathrm{H}), 3.40(\mathrm{~s}, 3 \mathrm{H}), 1.82(\mathrm{~s}, 3 \mathrm{H})$.

${ }^{13} \mathrm{C}$ NMR (101 MHz, DMSO) $\delta 169.27,166.33,155.59,139.53,132.51,132.14,131.42,130.40$, $130.05,128.22,127.57,125.62,119.36,116.69,51.80,22.44$.

HRMS (ESI) (m/z): calcd for $\mathrm{C}_{18} \mathrm{H}_{16} \mathrm{ClNO}_{4}[\mathrm{M}+\mathrm{Na}]^{+}: 368.0666$, found: 368.0665 .

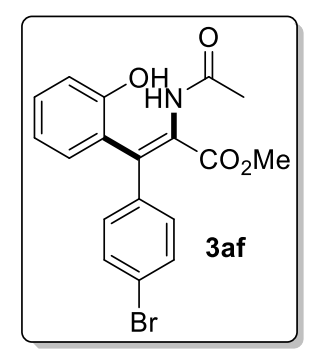

Methyl (Z)-2-acetamido-3-(4-bromophenyl)-3-(2-hydroxyphenyl)acrylate (3af)

This compound was obtained in $64 \%$ yield $(49.8 \mathrm{mg})$ as white solid. Mp: $116-117{ }^{\circ} \mathrm{C}$. Eluent: $\mathrm{PE} / \mathrm{EA}=1 / 1, \mathrm{R}_{\mathrm{f}}=0.26$.

${ }^{1} \mathrm{H}$ NMR $\left(400 \mathrm{MHz}, \mathrm{DMSO}-d_{6}\right) \delta 9.40(\mathrm{~s}, 1 \mathrm{H}), 9.25(\mathrm{~s}, 1 \mathrm{H}), 7.50-7.41(\mathrm{~m}, 2 \mathrm{H}), 7.16$ (ddd, $J$ $=8.2,7.2,1.8 \mathrm{~Hz}, 1 \mathrm{H}), 6.96-6.88(\mathrm{~m}, 3 \mathrm{H}), 6.84-6.78(\mathrm{~m}, 2 \mathrm{H}), 3.41(\mathrm{~s}, 3 \mathrm{H}), 1.82(\mathrm{~s}, 3 \mathrm{H})$.

${ }^{13} \mathrm{C}$ NMR (101 MHz, DMSO) $\delta 169.27,166.32,155.60,139.91,132.56,131.43,131.13,130.73$, 130.06, 127.52, 125.58, 120.75, 119.36, 116.69, 51.81, 22.44.

HRMS (ESI) $(\mathrm{m} / \mathrm{z})$ : calcd for $\mathrm{C}_{18} \mathrm{H}_{16} \mathrm{BrNO}_{4}[\mathrm{M}+\mathrm{Na}]^{+}:$412.0160, found: 412.0157 .

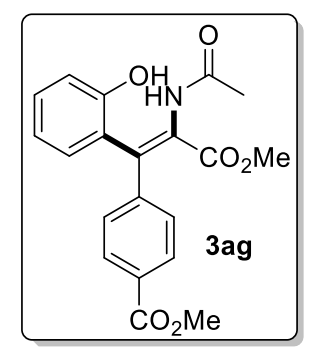




\section{yl)benzoate (3ag)}

This compound was obtained in $48 \%$ yield $\left(35.5 \mathrm{mg}\right.$ ) as white solid. Mp: $105-107{ }^{\circ} \mathrm{C}$. Eluent: $\mathrm{PE} / \mathrm{EA}=1 / 1, \mathrm{R}_{\mathrm{f}}=0.17$.

${ }^{1} \mathrm{H}$ NMR (400 MHz, DMSO- $\left.d_{6}\right) \delta 9.42(\mathrm{~s}, 1 \mathrm{H}), 9.32(\mathrm{~s}, 1 \mathrm{H}), 7.85(\mathrm{~d}, J=8.1 \mathrm{~Hz}, 2 \mathrm{H}), 7.20-$ $7.14(\mathrm{~m}, 1 \mathrm{H}), 7.10(\mathrm{~d}, J=8.0 \mathrm{~Hz}, 2 \mathrm{H}), 6.95(\mathrm{dd}, J=7.6,1.7 \mathrm{~Hz}, 1 \mathrm{H}), 6.81(\mathrm{t}, J=7.8 \mathrm{~Hz}, 2 \mathrm{H})$, $3.83(\mathrm{~s}, 3 \mathrm{H}), 3.37$ (s, 3H), $1.83(\mathrm{~s}, 3 \mathrm{H})$.

${ }^{13} \mathrm{C}$ NMR (101 MHz, DMSO) $\delta 168.83,166.00,165.70,155.15,145.18,132.09,130.98,129.64$, $128.60,128.47,128.03,127.76,124.92,118.91,116.19,52.04,51.32,21.95$.

HRMS (ESI) (m/z): calcd for $\mathrm{C}_{20} \mathrm{H}_{19} \mathrm{NO}_{6}[\mathrm{M}+\mathrm{Na}]^{+}: 392.1110$, found: 392.1109 .

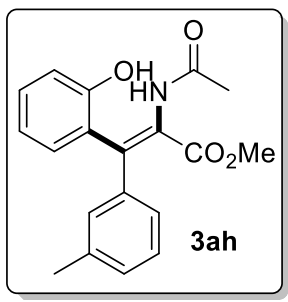

\section{Methyl (Z)-2-acetamido-3-(2-hydroxyphenyl)-3-(m-tolyl)acrylate (3ah)}

This compound was obtained in $71 \%$ yield $(46.2 \mathrm{mg})$ as white solid. Mp: $96-97{ }^{\circ} \mathrm{C}$. Eluent: $\mathrm{PE} / \mathrm{EA}=1 / 1, \mathrm{R}_{\mathrm{f}}=0.23$.

${ }^{1} \mathrm{H}$ NMR (400 MHz, DMSO- $\left.d_{6}\right) \delta 9.36(\mathrm{~s}, 1 \mathrm{H}), 9.12(\mathrm{~s}, 1 \mathrm{H}), 7.14$ (ddd, $J=9.2,5.0,1.9 \mathrm{~Hz}$, $2 \mathrm{H}), 7.04(\mathrm{~d}, J=7.6 \mathrm{~Hz}, 1 \mathrm{H}), 6.90(\mathrm{dd}, J=7.6,1.8 \mathrm{~Hz}, 1 \mathrm{H}), 6.86-6.73(\mathrm{~m}, 4 \mathrm{H}), 3.36(\mathrm{~s}, 3 \mathrm{H})$, $2.23(\mathrm{~s}, 3 \mathrm{H}), 1.82(\mathrm{~s}, 3 \mathrm{H})$.

${ }^{13} \mathrm{C}$ NMR (101 MHz, DMSO) $\delta 169.12,166.61,155.48,140.57,137.01,133.61,131.54,129.75$, $129.14,128.28,128.01,127.04,126.03,125.96,119.24,116.58,51.59,22.47,21.43$.

HRMS (ESI) (m/z): calcd for $\mathrm{C}_{19} \mathrm{H}_{19} \mathrm{NO}_{4}[\mathrm{M}+\mathrm{Na}]^{+}: 348.1212$, found: 348.1211 .

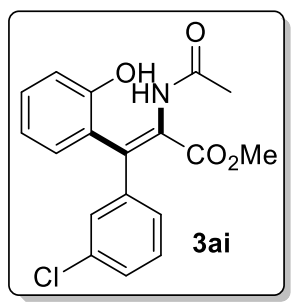

Methyl (Z)-2-acetamido-3-(3-chlorophenyl)-3-(2-hydroxyphenyl)acrylate (3ai)

This compound was obtained in $59 \%$ yield $(40.7 \mathrm{mg})$ as white solid. Mp: $86-87{ }^{\circ} \mathrm{C}$. Eluent: $\mathrm{PE} / \mathrm{EA}=1 / 1, \mathrm{R}_{\mathrm{f}}=0.26$.

${ }^{1} \mathrm{H}$ NMR (400 MHz, DMSO- $\left.d_{6}\right) \delta 9.44(\mathrm{~s}, 1 \mathrm{H}), 9.28(\mathrm{~s}, 1 \mathrm{H}), 7.33-7.26(\mathrm{~m}, 2 \mathrm{H}), 7.17$ (ddd, $J$ $=8.2,7.3,1.8 \mathrm{~Hz}, 1 \mathrm{H}), 7.03-6.91(\mathrm{~m}, 3 \mathrm{H}), 6.87-6.79(\mathrm{~m}, 2 \mathrm{H}), 3.40(\mathrm{~s}, 3 \mathrm{H}), 1.83(\mathrm{~s}, 3 \mathrm{H})$.

${ }^{13} \mathrm{C}$ NMR (101 MHz, DMSO) $\delta 169.30,166.23,155.57,142.77,132.73,131.88,131.40,130.14$, 130.08, 128.09, 127.43, 125.34, 119.43, 116.71, 51.79, 22.44 .

HRMS (ESI) (m/z): calcd for $\mathrm{C}_{18} \mathrm{H}_{16} \mathrm{ClNO}_{4}[\mathrm{M}+\mathrm{Na}]^{+}: 368.0666$, found: 368.0665 . 


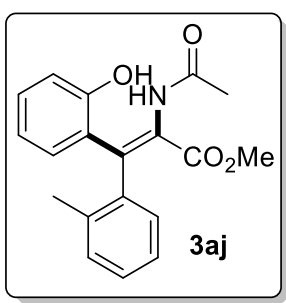

Methyl (Z)-2-acetamido-3-(2-hydroxyphenyl)-3-(o-tolyl)acrylate (3aj)

This compound was obtained in $67 \%$ yield $(43.6 \mathrm{mg})$ as white solid. $\mathrm{Mp}: 214-216^{\circ} \mathrm{C}$. Eluent: $\mathrm{PE} / \mathrm{EA}=1 / 1, \mathrm{R}_{\mathrm{f}}=0.20$.

${ }^{1} \mathrm{H}$ NMR (400 MHz, DMSO- $d_{6}$ ) $\delta 9.59$ (s, 1H), 9.08 (s, 1H), 7.11 (dddd, $J=10.9,8.6,5.6,2.8$ $\mathrm{Hz}, 4 \mathrm{H}), 7.01$ (dd, $J=7.2,1.8 \mathrm{~Hz}, 1 \mathrm{H}), 6.84$ (dd, $J=8.2,1.2 \mathrm{~Hz}, 1 \mathrm{H}), 6.77$ (dd, $J=7.7,1.9 \mathrm{~Hz}$, $1 \mathrm{H}), 6.71(\mathrm{td}, J=7.4,1.1 \mathrm{~Hz}, 1 \mathrm{H}), 3.26(\mathrm{~s}, 3 \mathrm{H}), 1.99$ (s, 3H), $1.85(\mathrm{~s}, 3 \mathrm{H})$.

${ }^{13} \mathrm{C}$ NMR (101 MHz, DMSO) $\delta 168.58,165.53,154.61,139.85,136.15,132.31,130.77,129.68$, $129.19,129.02,127.60,127.11,124.86,124.42,118.56,115.94,51.00,22.12,19.19$.

HRMS (ESI) (m/z): calcd for $\mathrm{C}_{19} \mathrm{H}_{19} \mathrm{NO}_{4}[\mathrm{M}+\mathrm{Na}]^{+}: 348.1212$, found: 348.1210 .<smiles>CC(=O)NC(C(=O)O)=C(c1ccccc1O)c1ccccc1F</smiles>

Methyl (E)-2-acetamido-3-(2-fluorophenyl)-3-(2-hydroxyphenyl)acrylate (3ak)

This compound was obtained in $52 \%$ yield $(34.2 \mathrm{mg})$ as white solid. Mp: $216-217^{\circ} \mathrm{C}$. Eluent: $\mathrm{PE} / \mathrm{EA}=1 / 1, \mathrm{R}_{\mathrm{f}}=0.21$.

${ }^{1} \mathrm{H}$ NMR (400 MHz, DMSO-d $\left.d_{6}\right) \delta 9.43(\mathrm{~s}, 1 \mathrm{H}), 9.28$ (s, 1H), 7.27 (dddd, $J=8.4,7.1,5.1,1.8$ $\mathrm{Hz}, 1 \mathrm{H}), 7.15$ (ddd, $J=8.2,7.2,1.8 \mathrm{~Hz}, 1 \mathrm{H}), 7.12-7.05$ (m, 2H), 6.97 (ddd, $J=17.1,7.6,1.8$ $\mathrm{Hz}, 2 \mathrm{H}), 6.85-6.76(\mathrm{~m}, 2 \mathrm{H}), 3.41(\mathrm{~s}, 3 \mathrm{H}), 1.84(\mathrm{~s}, 3 \mathrm{H})$.

${ }^{13} \mathrm{C} \mathrm{NMR}(101 \mathrm{MHz}, \mathrm{DMSO}) \delta 169.51,165.64,161.03,158.59,155.42,131.21,131.17,131.04$, $129.89,129.57,129.49,128.97,128.89,128.25,128.10,125.46,124.12,124.09,119.21,116.54$, $115.51,115.29,51.73,22.46$.

${ }^{19} \mathrm{~F}$ NMR (376 MHz, DMSO) $\delta-115.24$.

HRMS (ESI) (m/z): calcd for $\mathrm{C}_{18} \mathrm{H}_{16} \mathrm{FNO}_{4}[\mathrm{M}+\mathrm{Na}]^{+}: 352.0961$, found: 352.0960 .<smiles>CCCCCc1ccccc1-c1cccc(O)c1C(=O)OCC</smiles>

Ethyl (Z)-2-acetamido-3-(2-hydroxyphenyl)-3-phenylacrylate (3al)

This compound was obtained in $69 \%$ yield $(44.9 \mathrm{mg})$ as white solid. Mp: $114-115{ }^{\circ} \mathrm{C}$. Eluent: $\mathrm{PE} / \mathrm{EA}=1 / 1, \mathrm{R}_{\mathrm{f}}=0.19$. 
${ }^{1} \mathrm{H}$ NMR (400 MHz, DMSO- $\left.d_{6}\right) \delta 9.37(\mathrm{~s}, 1 \mathrm{H}), 9.12(\mathrm{~s}, 1 \mathrm{H}), 7.29-7.21(\mathrm{~m}, 3 \mathrm{H}), 7.15(\mathrm{td}, J=$ $7.7,1.8 \mathrm{~Hz}, 1 \mathrm{H}), 7.04-6.96(\mathrm{~m}, 2 \mathrm{H}), 6.92(\mathrm{dd}, J=7.6,1.8 \mathrm{~Hz}, 1 \mathrm{H}), 6.86-6.76(\mathrm{~m}, 2 \mathrm{H}), 3.82$ (q, $J=7.1 \mathrm{~Hz}, 2 \mathrm{H}), 1.82(\mathrm{~s}, 3 \mathrm{H}), 0.82(\mathrm{t}, J=7.1 \mathrm{~Hz}, 3 \mathrm{H})$.

${ }^{13} \mathrm{C}$ NMR (101 MHz, DMSO) $\delta 169.10,165.93,155.48,140.83,133.86,131.45,129.75,128.80$, 128.05, 127.50, 127.44, 126.09, 119.22, 116.57, 60.30, 22.47, 13.81 .

HRMS (ESI) (m/z): calcd for $\mathrm{C}_{19} \mathrm{H}_{19} \mathrm{NO}_{4}[\mathrm{M}+\mathrm{Na}]^{+}$: 348.1212, found: 348.1210 .

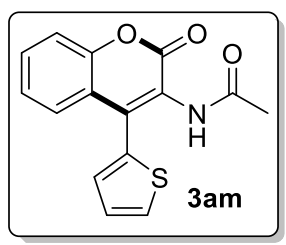

\section{$\mathrm{N}$-(2-oxo-4-(thiophen-2-yl)-2H-chromen-3-yl)acetamide (3am)}

This compound was obtained in 58\% yield $(33.1 \mathrm{mg})$ as light yellow solid. Mp: $203-205^{\circ} \mathrm{C}$. Eluent: $\mathrm{PE} / \mathrm{EA}=1 / 1, \mathrm{R}_{\mathrm{f}}=0.34$.

${ }^{1} \mathrm{H}$ NMR (400 MHz, DMSO- $\left.d_{6}\right) \delta 9.48(\mathrm{~s}, 1 \mathrm{H}), 7.90(\mathrm{dd}, J=5.1,1.2 \mathrm{~Hz}, 1 \mathrm{H}), 7.69-7.65(\mathrm{~m}$, 1H), 7.52 (ddd, $J=8.3,4.5,1.3 \mathrm{~Hz}, 2 \mathrm{H}), 7.39$ (d, $J=8.2 \mathrm{~Hz}, 1 \mathrm{H}), 7.36-7.34$ (m, 1H), 7.29 (dd, $J=5.0,3.6 \mathrm{~Hz}, 1 \mathrm{H}), 1.90(\mathrm{~s}, 3 \mathrm{H})$.

${ }^{13} \mathrm{C}$ NMR (101 MHz, DMSO) $\delta 170.10,158.46,152.08,142.99,132.41,131.66,131.06,129.78,127.89$, 127.53, 125.32, 122.88, 119.52, 117.14, 22.81.

HRMS (ESI) (m/z): calcd for $\mathrm{C}_{15} \mathrm{H}_{11} \mathrm{NO}_{3} \mathrm{~S}[\mathrm{M}+\mathrm{Na}]^{+}$308.0357, found: 308.0359.

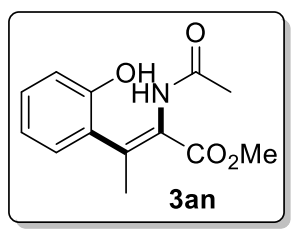

\section{Methyl (Z)-2-acetamido-3-(2-hydroxyphenyl)but-2-enoate (3an)}

This compound was obtained in $40 \%$ yield $(19.9 \mathrm{mg})$ as white solid. Mp: $147-148{ }^{\circ} \mathrm{C}$. Eluent: $\mathrm{PE} / \mathrm{EA}=1 / 1, \mathrm{R}_{\mathrm{f}}=0.17$.

${ }^{1} \mathrm{H}$ NMR (400 MHz, DMSO-d6) $\delta 9.61(\mathrm{~s}, 1 \mathrm{H}), 8.75$ (s, 1H), $7.18-7.07$ (m, 1H), 6.92 (dd, $J$ $=7.6,1.7 \mathrm{~Hz}, 1 \mathrm{H}), 6.87(\mathrm{dd}, J=8.2,1.1 \mathrm{~Hz}, 1 \mathrm{H}), 6.77(\mathrm{td}, J=7.4,1.1 \mathrm{~Hz}, 1 \mathrm{H}), 3.64(\mathrm{~s}, 3 \mathrm{H})$, $2.13(\mathrm{~s}, 3 \mathrm{H}), 1.72(\mathrm{~s}, 3 \mathrm{H})$.

${ }^{13} \mathrm{C}$ NMR (101 MHz, DMSO) $\delta 168.75,165.49,153.82,136.76,128.82,126.88,124.41,118.86$, 115.94, 51.33, 21.91, 19.36.

HRMS (ESI) (m/z): calcd for $\mathrm{C}_{13} \mathrm{H}_{15} \mathrm{NO}_{4}[\mathrm{M}+\mathrm{Na}]^{+} 272.0899$, found: 272.0896 .

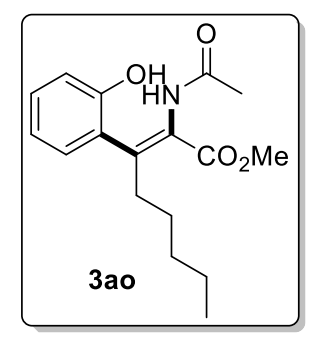

Methyl (Z)-2-acetamido-3-(2-hydroxyphenyl)oct-2-enoate (3ao) 
This compound was obtained in $67 \%$ yield $(40.9 \mathrm{mg})$ as white solid. $\mathrm{Mp}: 110-111{ }^{\circ} \mathrm{C}$. Eluent: $\mathrm{PE} / \mathrm{EA}=1 / 1, \mathrm{R}_{\mathrm{f}}=0.29$.

${ }^{1} \mathrm{H}$ NMR (400 MHz, DMSO- $\left.d_{6}\right) \delta 9.53(\mathrm{~s}, 1 \mathrm{H}), 8.67(\mathrm{~s}, 1 \mathrm{H}), 7.12$ (ddd, $J=8.1,7.2,1.8 \mathrm{~Hz}$, $1 \mathrm{H}), 6.88$ (ddd, $J=11.6,7.9,1.4 \mathrm{~Hz}, 2 \mathrm{H}), 6.78(\mathrm{td}, J=7.4,1.1 \mathrm{~Hz}, 1 \mathrm{H}), 3.63(\mathrm{~s}, 3 \mathrm{H}), 2.54$ (t, $J=7.2 \mathrm{~Hz}, 2 \mathrm{H}), 1.72$ (s, 3H), $1.25-1.14$ (m, 6H), $0.82-0.73$ (m, 3H).

${ }^{13} \mathrm{C}$ NMR (101 MHz, DMSO) $\delta 169.06,165.93,154.58,140.25,130.06,129.23,125.64,125.01$, 119.24, 116.29, 51.79, 32.08, 31.44, 27.94, 22.43, 22.29, 14.27 .

HRMS (ESI) (m/z): calcd for $\mathrm{C}_{17} \mathrm{H}_{23} \mathrm{NO}_{4}[\mathrm{M}+\mathrm{Na}]^{+} 328.1525$, found: 328.1527 .

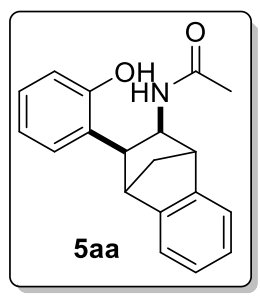

$N$-(3-(2-hydroxyphenyl)-1,2,3,4-tetrahydro-1,4-methanonaphthalen-2-yl)acetamide (5aa). This compound was obtained in $86 \%$ yield $(50.4 \mathrm{mg})$ as white solid. Mp: $113-114{ }^{\circ} \mathrm{C}$. Eluent: $\mathrm{PE} / \mathrm{EA}=1 / 1, \mathrm{R}_{\mathrm{f}}=0.26$.

${ }^{1} \mathrm{H}$ NMR (400 MHz, DMSO- $\left.d_{6}\right) \delta 9.11(\mathrm{~s}, 1 \mathrm{H}), 7.31-7.27(\mathrm{~m}, 1 \mathrm{H}), 7.24-7.19(\mathrm{~m}, 2 \mathrm{H}), 7.09$ $(\mathrm{dd}, J=5.3,3.1 \mathrm{~Hz}, 2 \mathrm{H}), 7.06-7.02(\mathrm{~m}, 1 \mathrm{H}), 6.83(\mathrm{td}, J=7.5,1.3 \mathrm{~Hz}, 1 \mathrm{H}), 6.73-6.65(\mathrm{~m}$, 2H), $4.29-4.18(\mathrm{~m}, 1 \mathrm{H}), 3.49(\mathrm{~d}, J=1.7 \mathrm{~Hz}, 1 \mathrm{H}), 3.13-3.05(\mathrm{~m}, 2 \mathrm{H}), 2.35(\mathrm{dt}, J=9.6,1.5$ $\mathrm{Hz}, 1 \mathrm{H}), 1.86$ (dt, $J=9.2,1.8 \mathrm{~Hz}, 1 \mathrm{H}), 1.52(\mathrm{~s}, 3 \mathrm{H})$.

${ }^{13} \mathrm{C} \mathrm{NMR}(101 \mathrm{MHz}, \mathrm{DMSO}) \delta 168.48,156.21,150.64,146.81,127.51,127.45,127.07,126.54$, 126.02, 122.19, 120.81, 119.14, 114.64, 52.73, 50.69, 46.57, 46.03, 44.02, 23.15.

HRMS (ESI) (m/z): calcd for $\mathrm{C}_{19} \mathrm{H}_{19} \mathrm{NO}_{2}[\mathrm{M}+\mathrm{Na}]^{+}: 316.1313$, found: 316.1309 .

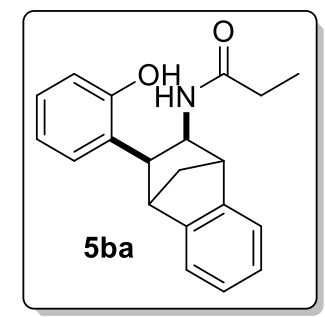

$N$-(3-(2-hydroxyphenyl)-1,2,3,4-tetrahydro-1,4-methanonaphthalen-2-yl)propionamide (5ba).

This compound was obtained in $72 \%$ yield $(44.2 \mathrm{mg})$ as white solid. Mp: $76-77{ }^{\circ} \mathrm{C}$. Eluent: $\mathrm{PE} / \mathrm{EA}=1 / 1, \mathrm{R}_{\mathrm{f}}=0.48$.

${ }^{1} \mathrm{H}$ NMR (400 MHz, DMSO- $d_{6}$ ) $\delta 9.07(\mathrm{~s}, 1 \mathrm{H}), 7.35-7.17(\mathrm{~m}, 3 \mathrm{H}), 7.15-6.95(\mathrm{~m}, 3 \mathrm{H}), 6.83$ (t, $J=7.4 \mathrm{~Hz}, 1 \mathrm{H}), 6.70(\mathrm{~d}, J=8.0 \mathrm{~Hz}, 1 \mathrm{H}), 6.53(\mathrm{~d}, J=9.6 \mathrm{~Hz}, 1 \mathrm{H}), 4.21(\mathrm{t}, J=8.9 \mathrm{~Hz}, 1 \mathrm{H})$, $3.49(\mathrm{~s}, 1 \mathrm{H}), 3.11(\mathrm{~s}, 2 \mathrm{H}), 2.36(\mathrm{~d}, J=9.6 \mathrm{~Hz}, 1 \mathrm{H}), 1.91-1.64(\mathrm{~m}, 3 \mathrm{H}), 0.70(\mathrm{t}, J=7.6 \mathrm{~Hz}$, $3 \mathrm{H})$.

${ }^{13} \mathrm{C}$ NMR (101 MHz, DMSO) $\delta 172.22,156.23,150.70,146.81,127.50,127.43,127.05,126.54$, 126.03, 122.22, 120.81, 119.16, 114.76, 52.40, 50.57, 46.58, 46.07, 44.08, 29.16, 10.31 . HRMS (ESI) (m/z): calcd for $\mathrm{C}_{20} \mathrm{H}_{21} \mathrm{NO}_{2}[\mathrm{M}+\mathrm{Na}]^{+}: 330.1470$, found: 330.1472 


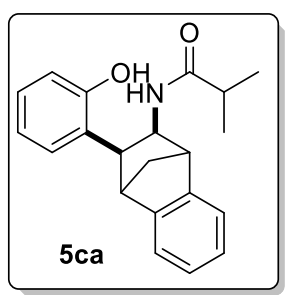

$N$-(3-(2-hydroxyphenyl)-1,2,3,4-tetrahydro-1,4-methanonaphthalen-2-yl)isobutyramide (5ca).

This compound was obtained in $68 \%$ yield $(43.7 \mathrm{mg})$ as white solid. Mp: $188-189{ }^{\circ} \mathrm{C}$. Eluent: $\mathrm{PE} / \mathrm{EA}=1 / 1, \mathrm{R}_{\mathrm{f}}=0.63$.

${ }^{1} \mathrm{H}$ NMR (400 MHz, DMSO- $\left.d_{6}\right) \delta 9.04(\mathrm{~s}, 1 \mathrm{H}), 7.40-7.27(\mathrm{~m}, 1 \mathrm{H}), 7.22(\mathrm{q}, J=3.6 \mathrm{~Hz}, 2 \mathrm{H})$, $7.10(\mathrm{dd}, J=5.3,3.1 \mathrm{~Hz}, 2 \mathrm{H}), 7.03(\mathrm{t}, J=7.7 \mathrm{~Hz}, 1 \mathrm{H}), 6.83(\mathrm{t}, J=7.4 \mathrm{~Hz}, 1 \mathrm{H}), 6.70(\mathrm{~d}, J=7.9$ $\mathrm{Hz}, 1 \mathrm{H}), 6.48(\mathrm{~d}, J=9.5 \mathrm{~Hz}, 1 \mathrm{H}), 4.20(\mathrm{t}, J=8.8 \mathrm{~Hz}, 1 \mathrm{H}), 3.48(\mathrm{~s}, 1 \mathrm{H}), 3.10(\mathrm{~d}, J=7.8 \mathrm{~Hz}$, $2 \mathrm{H}), 2.38(\mathrm{~d}, J=9.6 \mathrm{~Hz}, 1 \mathrm{H}), 2.14-1.98(\mathrm{~m}, 1 \mathrm{H}), 1.86(\mathrm{~d}, J=9.5 \mathrm{~Hz}, 1 \mathrm{H}), 0.80(\mathrm{~d}, J=6.7 \mathrm{~Hz}$, $3 \mathrm{H}), 0.56(\mathrm{~d}, J=6.9 \mathrm{~Hz}, 3 \mathrm{H})$.

${ }^{13} \mathrm{C}$ NMR (101 MHz, DMSO) $\delta 175.21,156.27,150.77,146.79,127.50,127.41,127.06,126.54$, 126.02, 122.26, 120.78, 119.20, 114.94, 52.12, 50.52, 46.67, 46.16, 44.12, 34.20, 20.14, 19.16. HRMS (ESI) (m/z): calcd for $\mathrm{C}_{21} \mathrm{H}_{23} \mathrm{NO}_{2}[\mathrm{M}+\mathrm{Na}]^{+}: 344.1626$, found: 344.1629

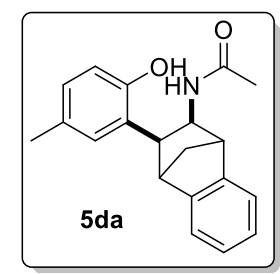

$N$-(3-(2-hydroxy-5-methylphenyl)-1,2,3,4-tetrahydro-1,4-methanonaphthalen-2yl)acetamide (5da).

This compound was obtained in $82 \%$ yield $(50.4 \mathrm{mg})$ as white solid. Mp: $87-88{ }^{\circ} \mathrm{C}$. Eluent: $\mathrm{PE} / \mathrm{EA}=1 / 1, \mathrm{R}_{\mathrm{f}}=0.35$.

${ }^{1} \mathrm{H}$ NMR $\left(400 \mathrm{MHz}\right.$, DMSO- $\left.d_{6}\right) \delta 8.82(\mathrm{~s}, 1 \mathrm{H}), 7.28(\mathrm{dd}, J=5.4,3.0 \mathrm{~Hz}, 1 \mathrm{H}), 7.21(\mathrm{dd}, J=5.3$, $3.1 \mathrm{~Hz}, 1 \mathrm{H}), 7.09(\mathrm{dd}, J=5.3,3.1 \mathrm{~Hz}, 2 \mathrm{H}), 7.03(\mathrm{~d}, J=2.2 \mathrm{~Hz}, 1 \mathrm{H}), 6.84(\mathrm{dd}, J=8.0,2.1 \mathrm{~Hz}$, $1 \mathrm{H}), 6.69(\mathrm{~d}, J=9.6 \mathrm{~Hz}, 1 \mathrm{H}), 6.60(\mathrm{~d}, J=8.0 \mathrm{~Hz}, 1 \mathrm{H}), 4.20(\mathrm{t}, J=9.0 \mathrm{~Hz}, 1 \mathrm{H}), 3.50(\mathrm{~s}, 1 \mathrm{H})$, $3.09(\mathrm{~s}, 1 \mathrm{H}), 3.03(\mathrm{~d}, J=8.3 \mathrm{~Hz}, 1 \mathrm{H}), 2.32(\mathrm{~d}, J=9.5 \mathrm{~Hz}, 1 \mathrm{H}), 2.26(\mathrm{~s}, 3 \mathrm{H}), 1.88-1.83(\mathrm{~m}$, $1 \mathrm{H}), 1.53(\mathrm{~s}, 3 \mathrm{H})$.

${ }^{13} \mathrm{C}$ NMR (101 MHz, DMSO) $\delta 168.58,153.93,150.61,146.84,128.15,127.79,127.25,126.75$, 126.52, 126.01, 122.15, 120.82, 114.55, 52.77, 50.73, 46.49, 46.05, 44.03, 23.21, 21.16. HRMS (ESI) (m/z): calcd for $\mathrm{C}_{20} \mathrm{H}_{21} \mathrm{NO}_{2} \quad[\mathrm{M}+\mathrm{Na}]^{+}: 330.1470$, found: 330.1472

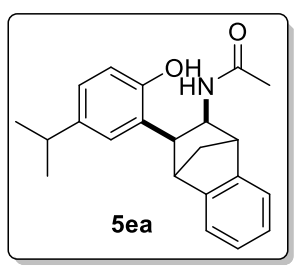


$N$-(3-(2-hydroxy-5-isopropylphenyl)-1,2,3,4-tetrahydro-1,4-methanonaphthalen-2yl)acetamide (5ea).

This compound was obtained in $78 \%$ yield $(52.3 \mathrm{mg})$ as white solid. Mp: $81-82{ }^{\circ} \mathrm{C}$. Eluent: $\mathrm{PE} / \mathrm{EA}=1 / 1, \mathrm{R}_{\mathrm{f}}=0.35$.

${ }^{1} \mathrm{H}$ NMR $\left(400 \mathrm{MHz}, \mathrm{DMSO}-d_{6}\right) \delta 8.82(\mathrm{~s}, 1 \mathrm{H}), 7.29(\mathrm{dd}, J=5.3,3.1 \mathrm{~Hz}, 1 \mathrm{H}), 7.22(\mathrm{dd}, J=5.3$, $3.1 \mathrm{~Hz}, 1 \mathrm{H}), 7.09(\mathrm{dd}, J=5.3,3.1 \mathrm{~Hz}, 2 \mathrm{H}), 7.06(\mathrm{~d}, J=2.1 \mathrm{~Hz}, 1 \mathrm{H}), 6.89(\mathrm{dd}, J=8.2,2.2 \mathrm{~Hz}$, $1 \mathrm{H}), 6.67(\mathrm{~d}, J=9.4 \mathrm{~Hz}, 1 \mathrm{H}), 6.63(\mathrm{~d}, J=8.2 \mathrm{~Hz}, 1 \mathrm{H}), 4.15(\mathrm{t}, J=8.8 \mathrm{~Hz}, 1 \mathrm{H}), 3.46(\mathrm{~s}, 1 \mathrm{H})$, $3.14(\mathrm{~s}, 1 \mathrm{H}), 3.10$ (dd, $J=8.2,1.6 \mathrm{~Hz}, 1 \mathrm{H}), 2.84(\mathrm{p}, J=6.9 \mathrm{~Hz}, 1 \mathrm{H}), 2.37$ (d, $J=9.5 \mathrm{~Hz}, 1 \mathrm{H})$, $1.91-1.85(\mathrm{~m}, 1 \mathrm{H}), 1.51(\mathrm{~s}, 3 \mathrm{H}), 1.21(\mathrm{dd}, J=6.8,1.3 \mathrm{~Hz}, 6 \mathrm{H})$.

${ }^{13} \mathrm{C}$ NMR (101 MHz, DMSO) $\delta 168.50,154.00,150.77,146.78,138.65,126.59,126.54,126.00$, $125.74,124.67,122.18,120.79,114.64,52.94,50.38,46.96,46.21,43.93,33.30,24.91,24.54$, 23.17.

HRMS (ESI) (m/z): calcd for $\mathrm{C}_{22} \mathrm{H}_{25} \mathrm{NO}_{2}[\mathrm{M}+\mathrm{Na}]^{+}: 358.1783$, found: 358.1781

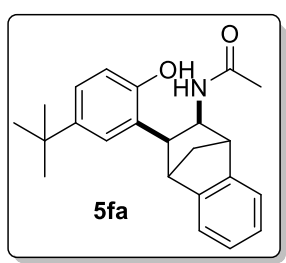

$N$-(3-(5-(tert-butyl)-2-hydroxyphenyl)-1,2,3,4-tetrahydro-1,4-methanonaphthalen-2yl)acetamide (5fa).

This compound was obtained in $76 \%$ yield $(53.1 \mathrm{mg})$ as white solid. Mp: $76-77{ }^{\circ} \mathrm{C}$. Eluent: $\mathrm{PE} / \mathrm{EA}=1 / 1, \mathrm{R}_{\mathrm{f}}=0.53$.

${ }^{1} \mathrm{H}$ NMR $\left(400 \mathrm{MHz}\right.$, DMSO- $\left.d_{6}\right) \delta 8.83(\mathrm{~s}, 1 \mathrm{H}), 7.30(\mathrm{dd}, J=5.3,3.1 \mathrm{~Hz}, 1 \mathrm{H}), 7.27-7.15(\mathrm{~m}$, 2H), $7.09(\mathrm{dd}, J=5.3,3.1 \mathrm{~Hz}, 2 \mathrm{H}), 7.03(\mathrm{dd}, J=8.4,2.4 \mathrm{~Hz}, 1 \mathrm{H}), 6.64(\mathrm{dd}, J=16.0,8.8 \mathrm{~Hz}$, 2H), $4.13(\mathrm{t}, J=8.7 \mathrm{~Hz}, 1 \mathrm{H}), 3.45(\mathrm{~s}, 1 \mathrm{H}), 3.21-3.07(\mathrm{~m}, 2 \mathrm{H}), 2.38$ (d, $J=9.5 \mathrm{~Hz}, 1 \mathrm{H}), 1.92$ $-1.86(\mathrm{~m}, 1 \mathrm{H}), 1.50(\mathrm{~s}, 3 \mathrm{H}), 1.29(\mathrm{~s}, 9 \mathrm{H})$.

${ }^{13} \mathrm{C}$ NMR (101 MHz, DMSO) $\delta 168.45,153.63,150.81,146.75,140.94,126.55,126.07,126.00$, 124.27, 123.97, 122.18, 120.77, 114.40, 53.01, 50.24, 47.13, 46.21, 43.97, 34.28, 31.99, 31.77, 23.16 .

HRMS (ESI) (m/z): calcd for $\mathrm{C}_{23} \mathrm{H}_{27} \mathrm{NO}_{2}[\mathrm{M}+\mathrm{Na}]^{+}: 372.1939$, found: 372.1940

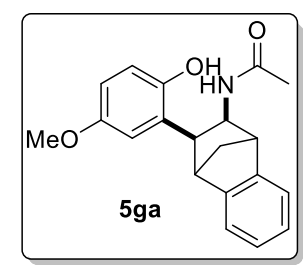

$N$-(3-(2-hydroxy-5-methoxyphenyl)-1,2,3,4-tetrahydro-1,4-methanonaphthalen-2yl)acetamide (5ga).

This compound was obtained in $62 \%$ yield $(40.1 \mathrm{mg})$ as white solid. Mp: $141-142{ }^{\circ} \mathrm{C}$. Eluent: $\mathrm{PE} / \mathrm{EA}=1 / 1, \mathrm{R}_{\mathrm{f}}=0.24$.

${ }^{1} \mathrm{H}$ NMR $\left(500 \mathrm{MHz}\right.$, DMSO- $\left.d_{6}\right) \delta 8.58(\mathrm{~s}, 1 \mathrm{H}), 7.29(\mathrm{dd}, J=5.3,3.1 \mathrm{~Hz}, 1 \mathrm{H}), 7.22(\mathrm{dd}, J=5.3$, $3.1 \mathrm{~Hz}, 1 \mathrm{H}), 7.10(\mathrm{dd}, J=5.3,3.1 \mathrm{~Hz}, 2 \mathrm{H}), 6.83(\mathrm{~d}, J=9.7 \mathrm{~Hz}, 1 \mathrm{H}), 6.78(\mathrm{~d}, J=1.6 \mathrm{~Hz}, 1 \mathrm{H})$, 
$6.63(\mathrm{~d}, J=1.6 \mathrm{~Hz}, 2 \mathrm{H}), 4.29-4.11(\mathrm{~m}, 1 \mathrm{H}), 3.72(\mathrm{~s}, 3 \mathrm{H}), 3.49(\mathrm{~d}, J=1.7 \mathrm{~Hz}, 1 \mathrm{H}), 3.10(\mathrm{~s}$, $1 \mathrm{H}), 3.05(\mathrm{dd}, J=8.2,1.7 \mathrm{~Hz}, 1 \mathrm{H}), 2.35(\mathrm{~d}, J=9.5 \mathrm{~Hz}, 1 \mathrm{H}), 1.90-1.84(\mathrm{~m}, 1 \mathrm{H}), 1.54(\mathrm{~s}, 3 \mathrm{H})$. ${ }^{13} \mathrm{C}$ NMR (126 MHz, DMSO) $\delta 168.67,152.38,150.53,149.98,146.78,128.25,126.57,126.06$, 122.20, 120.84, 115.09, 114.08, 111.69, 55.63, 52.91, 50.63, 46.59, 46.01, 44.24, 23.14 .

HRMS (ESI) (m/z): calcd for $\mathrm{C}_{20} \mathrm{H}_{21} \mathrm{NO}_{3}[\mathrm{M}+\mathrm{Na}]^{+}: 346.1419$, found: 346.1423

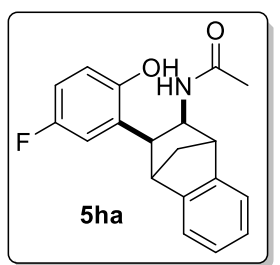

\section{$N$-(3-(5-fluoro-2-hydroxyphenyl)-1,2,3,4-tetrahydro-1,4-methanonaphthalen-2-} yl)acetamide (5ha).

This compound was obtained in $78 \%$ yield $\left(48.5 \mathrm{mg}\right.$ ) as white solid. Mp: $207-208{ }^{\circ} \mathrm{C}$. Eluent: $\mathrm{PE} / \mathrm{EA}=1 / 1, \mathrm{R}_{\mathrm{f}}=0.35$.

${ }^{1} \mathrm{H}$ NMR $\left(400 \mathrm{MHz}\right.$, DMSO- $\left.d_{6}\right) \delta 9.10(\mathrm{~s}, 1 \mathrm{H}), 7.29(\mathrm{dd}, J=5.3,3.0 \mathrm{~Hz}, 1 \mathrm{H}), 7.22(\mathrm{dd}, J=5.3$, $3.1 \mathrm{~Hz}, 1 \mathrm{H}), 7.10(\mathrm{dd}, J=5.3,3.1 \mathrm{~Hz}, 2 \mathrm{H}), 6.99(\mathrm{dd}, J=10.1,3.1 \mathrm{~Hz}, 1 \mathrm{H}), 6.93(\mathrm{~d}, J=9.9 \mathrm{~Hz}$, $1 \mathrm{H}), 6.84$ (td, $J=8.6,3.1 \mathrm{~Hz}, 1 \mathrm{H}), 6.66(\mathrm{dd}, J=8.8,5.0 \mathrm{~Hz}, 1 \mathrm{H}), 4.26(\mathrm{t}, J=9.1 \mathrm{~Hz}, 1 \mathrm{H}), 3.48$ (s, 1H), $3.12-3.01(\mathrm{~m}, 2 \mathrm{H}), 2.33(\mathrm{~d}, J=9.6 \mathrm{~Hz}, 1 \mathrm{H}), 1.90-1.81(\mathrm{~m}, 1 \mathrm{H}), 1.53(\mathrm{~s}, 3 \mathrm{H})$.

${ }^{13} \mathrm{C}$ NMR (101 MHz, DMSO) $\delta 168.51,157.03,154.72,152.44,150.34,146.75,129.10,129.03$, $126.59,126.12,122.21,120.88,115.02,114.94,114.61,114.38,113.37,113.14,52.84,50.65$, 46.52, 45.92, 44.23, 23.07.

${ }^{19} \mathrm{~F}$ NMR (376 MHz, DMSO) $\delta-125.47$.

HRMS (ESI) (m/z): calcd for C19H18FNO2 [M+Na] $]^{+}: 334.1219$, found: 334.1213.

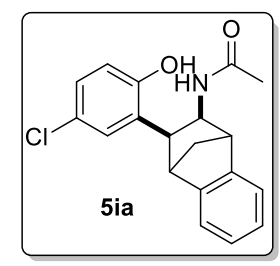

\section{$N$-(3-(5-chloro-2-hydroxyphenyl)-1,2,3,4-tetrahydro-1,4-methanonaphthalen-2-} yl)acetamide (5ia).

This compound was obtained in $64 \%$ yield $(41.9 \mathrm{mg})$ as white solid. $\mathrm{Mp}: 132-133{ }^{\circ} \mathrm{C}$. Eluent: $\mathrm{PE} / \mathrm{EA}=1 / 1, \mathrm{R}_{\mathrm{f}}=0.31$.

${ }^{1} \mathrm{H}$ NMR $\left(400 \mathrm{MHz}, \mathrm{DMSO}-d_{6}\right) \delta 9.45(\mathrm{~s}, 1 \mathrm{H}), 7.29(\mathrm{dd}, J=5.4,3.0 \mathrm{~Hz}, 1 \mathrm{H}), 7.22(\mathrm{dd}, J=5.4$, $3.1 \mathrm{~Hz}, 1 \mathrm{H}), 7.17(\mathrm{~d}, J=2.6 \mathrm{~Hz}, 1 \mathrm{H}), 7.08(\mathrm{ddd}, J=13.2,6.9,2.8 \mathrm{~Hz}, 3 \mathrm{H}), 6.99(\mathrm{~d}, J=10.0$ $\mathrm{Hz}, 1 \mathrm{H}), 6.69(\mathrm{~d}, J=8.6 \mathrm{~Hz}, 1 \mathrm{H}), 4.27(\mathrm{t}, J=9.2 \mathrm{~Hz}, 1 \mathrm{H}), 3.50(\mathrm{~s}, 1 \mathrm{H}), 3.07(\mathrm{~s}, 1 \mathrm{H}), 3.02(\mathrm{~d}, J$ $=8.3 \mathrm{~Hz}, 1 \mathrm{H}), 2.32(\mathrm{~d}, J=9.6 \mathrm{~Hz}, 1 \mathrm{H}), 1.90-1.81(\mathrm{~m}, 1 \mathrm{H}), 1.54(\mathrm{~s}, 3 \mathrm{H})$.

${ }^{13} \mathrm{C}$ NMR (101 MHz, DMSO) $\delta 168.46,155.25,150.27,146.78,129.57,127.44,127.07,126.58$, 126.13, 122.56, 122.17, 120.94, 115.81, 52.75, 50.75, 46.32, 45.87, 44.21, 23.10.

HRMS (ESI) (m/z): calcd for $\mathrm{C}_{19} \mathrm{H}_{18} \mathrm{ClNO}_{2}[\mathrm{M}+\mathrm{Na}]^{+}: 350.0924$, found: 350.0915 . 


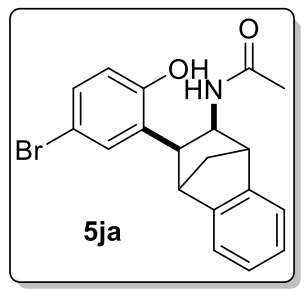

$\mathrm{N}$-(3-(5-bromo-2-hydroxyphenyl)-1,2,3,4-tetrahydro-1,4-methanonaphthalen-2yl)acetamide (5ja).

This compound was obtained in $67 \%$ yield $(49.7 \mathrm{mg})$ as white solid. $\mathrm{Mp}: 117-118{ }^{\circ} \mathrm{C}$. Eluent: $\mathrm{PE} / \mathrm{EA}=1 / 1, \mathrm{R}_{\mathrm{f}}=0.34$.

${ }^{1} \mathrm{H}$ NMR (400 MHz, DMSO- $\left.d_{6}\right) \delta 9.49(\mathrm{~s}, 1 \mathrm{H}), 7.28(\mathrm{q}, J=2.9,2.2 \mathrm{~Hz}, 2 \mathrm{H}), 7.20$ (ddd, $J=$ $11.1,6.7,2.6 \mathrm{~Hz}, 2 \mathrm{H}), 7.09$ (dd, $J=5.3,3.1 \mathrm{~Hz}, 2 \mathrm{H}), 7.02$ (d, $J=10.0 \mathrm{~Hz}, 1 \mathrm{H}), 6.66$ (d, $J=8.5$ $\mathrm{Hz}, 1 \mathrm{H}), 4.27$ (t, $J=9.2 \mathrm{~Hz}, 1 \mathrm{H}), 3.50$ (s, 1H), 3.07 (s, 1H), 3.02 (d, $J=8.3 \mathrm{~Hz}, 1 \mathrm{H}), 2.32$ (d, $J$ $=9.6 \mathrm{~Hz}, 1 \mathrm{H}), 1.86(\mathrm{~d}, J=9.6 \mathrm{~Hz}, 1 \mathrm{H}), 1.55(\mathrm{~s}, 3 \mathrm{H})$.

${ }^{13} \mathrm{C}$ NMR (101 MHz, DMSO) $\delta 168.49,155.72,150.24,146.78,130.17,130.14,130.00,126.57$, 126.13, 122.16, 120.95, 116.42, 110.39, 52.78, 50.76, 46.32, 45.87, 44.21, 23.11.

HRMS (ESI) (m/z): calcd for $\mathrm{C}_{19} \mathrm{H}_{18} \mathrm{BrNO}_{2}[\mathrm{M}+\mathrm{H}]^{+}: 372.0599$, found: 372.0601 .

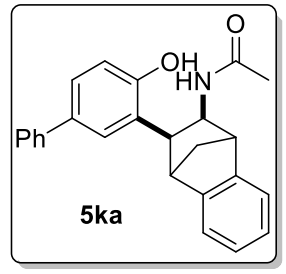

$N$-(3-(4-hydroxy-[1,1'-biphenyl]-3-yl)-1,2,3,4-tetrahydro-1,4-methanonaphthalen-2yl)acetamide (ka).

This compound was obtained in $58 \%$ yield $(42.8 \mathrm{mg})$ as white solid. $\mathrm{Mp}: 181-182{ }^{\circ} \mathrm{C}$. Eluent: $\mathrm{PE} / \mathrm{EA}=1 / 1, \mathrm{R}_{\mathrm{f}}=0.26$.

${ }^{1} \mathrm{H}$ NMR (400 MHz, DMSO- $\left.d_{6}\right) \delta 9.32(\mathrm{~s}, 1 \mathrm{H}), 7.69(\mathrm{~d}, J=7.6 \mathrm{~Hz}, 2 \mathrm{H}), 7.44(\mathrm{dd}, J=15.1,7.5$ $\mathrm{Hz}, 3 \mathrm{H}), 7.30(\mathrm{td}, J=22.2,20.2,10.6 \mathrm{~Hz}, 4 \mathrm{H}), 7.15-7.06(\mathrm{~m}, 2 \mathrm{H}), 6.92(\mathrm{~d}, J=9.9 \mathrm{~Hz}, 1 \mathrm{H})$, $6.79(\mathrm{~d}, J=8.3 \mathrm{~Hz}, 1 \mathrm{H}), 4.28(\mathrm{t}, J=9.0 \mathrm{~Hz}, 1 \mathrm{H}), 3.63(\mathrm{~s}, 1 \mathrm{H}), 3.13(\mathrm{~d}, J=8.6 \mathrm{~Hz}, 2 \mathrm{H}), 2.45$ $(\mathrm{d}, J=10.2 \mathrm{~Hz}, 1 \mathrm{H}), 1.95-1.87(\mathrm{~m}, 1 \mathrm{H}), 1.53(\mathrm{~s}, 3 \mathrm{H})$.

${ }^{13} \mathrm{C}$ NMR (101 MHz, DMSO) $\delta 168.43,156.09,150.72,146.88,141.39,130.97,129.35,129.16$, $127.50,126.81,126.67,126.64,126.57,126.19,126.04,125.75,122.19,120.84,114.97,52.80$, 50.71, 46.61, 46.16, 44.13, 23.17.

HRMS (ESI) (m/z): calcd for $\mathrm{C}_{25} \mathrm{H}_{23} \mathrm{NO}_{2}[\mathrm{M}+\mathrm{Na}]^{+}$: 392.1626 , found: 392.1623 .

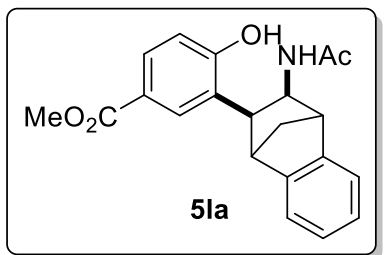

Methyl

3-(3-acetamido-1,2,3,4-tetrahydro-1,4-methanonaphthalen-2-yl)-4hydroxybenzoate (5la). 
This compound was obtained in $41 \%$ yield $(28.8 \mathrm{mg})$ as white solid. Mp: $98-99{ }^{\circ} \mathrm{C}$. Eluent: $\mathrm{PE} / \mathrm{EA}=1 / 1, \mathrm{R}_{\mathrm{f}}=0.19$.

${ }^{1} \mathrm{H}$ NMR $\left(400 \mathrm{MHz}, \mathrm{DMSO}-d_{6}\right) \delta 10.19(\mathrm{~s}, 1 \mathrm{H}), 7.86(\mathrm{~d}, J=2.1 \mathrm{~Hz}, 1 \mathrm{H}), 7.72(\mathrm{dd}, J=8.4,2.1$ $\mathrm{Hz}, 1 \mathrm{H}), 7.29(\mathrm{dd}, J=5.6,2.9 \mathrm{~Hz}, 1 \mathrm{H}), 7.28-7.21(\mathrm{~m}, 1 \mathrm{H}), 7.15-7.06(\mathrm{~m}, 2 \mathrm{H}), 6.90(\mathrm{~d}, J=$ $10.1 \mathrm{~Hz}, 1 \mathrm{H}), 6.78(\mathrm{~d}, J=8.4 \mathrm{~Hz}, 1 \mathrm{H}), 4.30(\mathrm{t}, J=9.1 \mathrm{~Hz}, 1 \mathrm{H}), 3.82(\mathrm{~s}, 3 \mathrm{H}), 3.55(\mathrm{~s}, 1 \mathrm{H}), 3.10$ $-2.98(\mathrm{~m}, 2 \mathrm{H}), 2.35(\mathrm{~d}, J=9.6 \mathrm{~Hz}, 1 \mathrm{H}), 1.91(\mathrm{~d}, J=9.4 \mathrm{~Hz}, 1 \mathrm{H}), 1.50(\mathrm{~s}, 3 \mathrm{H})$.

${ }^{13} \mathrm{C}$ NMR (101 MHz, DMSO) $\delta$ 167.90, 166.48, 160.62, 149.74, 146.31, 129.24, 128.63, 126.94, 126.09, 125.65, 121.65, 120.52, 119.71, 113.74, 52.07, 51.55, 50.40, 45.62, 45.30, 43.59, 22.56. HRMS (ESI) (m/z): calcd for $\mathrm{C}_{21} \mathrm{H}_{21} \mathrm{NO}_{4}[\mathrm{M}+\mathrm{Na}]^{+}:$374.1368, found: 374.1366 .

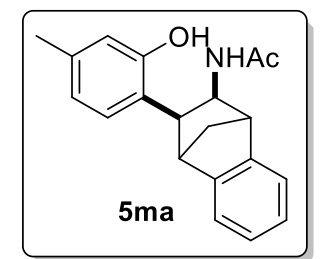

$N$-(3-(2-hydroxy-4-methylphenyl)-1,2,3,4-tetrahydro-1,4-methanonaphthalen-2yl)acetamide (5ma).

This compound was obtained in $77 \%$ yield $(47.3 \mathrm{mg})$ as white solid. $\mathrm{Mp}: 170-171{ }^{\circ} \mathrm{C}$. Eluent: $\mathrm{PE} / \mathrm{EA}=1 / 1, \mathrm{R}_{\mathrm{f}}=0.37$.

${ }^{1} \mathrm{H}$ NMR $\left(400 \mathrm{MHz}\right.$, DMSO- $\left.d_{6}\right) \delta 8.98(\mathrm{~s}, 1 \mathrm{H}), 7.28(\mathrm{dd}, J=5.4,3.0 \mathrm{~Hz}, 1 \mathrm{H}), 7.21(\mathrm{dd}, J=5.4$, $3.1 \mathrm{~Hz}, 1 \mathrm{H}), 7.14-7.03(\mathrm{~m}, 3 \mathrm{H}), 6.71-6.61(\mathrm{~m}, 2 \mathrm{H}), 6.53(\mathrm{~d}, J=1.8 \mathrm{~Hz}, 1 \mathrm{H}), 4.18(\mathrm{t}, J=9.0$ $\mathrm{Hz}, 1 \mathrm{H}), 3.46$ (s, 1H), 3.09 (s, 1H), $3.05-2.96(\mathrm{~m}, 1 \mathrm{H}), 2.30$ (d, $J=9.5 \mathrm{~Hz}, 1 \mathrm{H}), 2.20(\mathrm{~s}, 3 \mathrm{H})$, $1.89-1.80(\mathrm{~m}, 1 \mathrm{H}), 1.54(\mathrm{~s}, 3 \mathrm{H})$.

${ }^{13} \mathrm{C}$ NMR (101 MHz, DMSO) $\delta 168.52,156.08,150.61,146.83,136.47,127.33,126.51,126.00$, 123.94, 122.16, 120.81, 119.90, 115.36, 52.71, 50.72, 46.66, 45.99, 43.68, 23.24, 21.27.

HRMS (ESI) (m/z): calcd for $\mathrm{C}_{20} \mathrm{H}_{21} \mathrm{NO}_{2}[\mathrm{M}+\mathrm{Na}]^{+}: 330.1470$, found: 330.1466 .

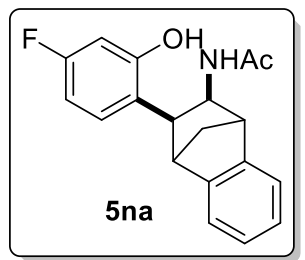

$N$-(3-(4-fluoro-2-hydroxyphenyl)-1,2,3,4-tetrahydro-1,4-methanonaphthalen-2yl)acetamide (5na).

This compound was obtained in $52 \%$ yield $(32.4 \mathrm{mg})$ as white solid. $\mathrm{Mp}: 156-157^{\circ} \mathrm{C}$. Eluent: $\mathrm{PE} / \mathrm{EA}=1 / 1, \mathrm{R}_{\mathrm{f}}=0.29$.

${ }^{1} \mathrm{H}$ NMR $\left(400 \mathrm{MHz}, \mathrm{DMSO}-d_{6}\right) \delta 9.66(\mathrm{~s}, 1 \mathrm{H}), 7.27(\mathrm{dd}, J=5.3,3.1 \mathrm{~Hz}, 1 \mathrm{H}), 7.20(\mathrm{dd}, J=5.3$, $3.1 \mathrm{~Hz}, 1 \mathrm{H}), 7.13-6.98(\mathrm{~m}, 4 \mathrm{H}), 6.60-6.45(\mathrm{~m}, 2 \mathrm{H}), 4.29(\mathrm{t}, J=9.0 \mathrm{~Hz}, 1 \mathrm{H}), 3.72(\mathrm{~s}, 1 \mathrm{H})$, $3.15-3.05(\mathrm{~m}, 2 \mathrm{H}), 1.86(\mathrm{~d}, J=9.6 \mathrm{~Hz}, 1 \mathrm{H}), 1.53(\mathrm{~s}, 3 \mathrm{H})$.

${ }^{13} \mathrm{C}$ NMR (101 MHz, DMSO) $\delta 168.53,163.61,161.20,158.10,158.01,151.13,146.45,128.06$, $127.94,126.58,126.06,122.27,120.62,53.08,50.22,46.32,46.25,44.24,23.05$.

${ }^{19} \mathrm{~F}$ NMR (376 MHz, DMSO) $\delta-116.00$.

HRMS (ESI) (m/z): calcd for $\mathrm{C}_{19} \mathrm{H}_{18} \mathrm{FNO}_{2}[\mathrm{M}+\mathrm{Na}]^{+}: 334.1219$, found: 334.1215 . 


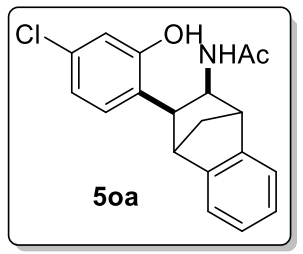

$N$-(3-(4-chloro-2-hydroxyphenyl)-1,2,3,4-tetrahydro-1,4-methanonaphthalen-2yl)acetamide (5oa).

This compound was obtained in $61 \%$ yield $(39.9 \mathrm{mg})$ as white solid. Mp: $187-189{ }^{\circ} \mathrm{C}$. Eluent: $\mathrm{PE} / \mathrm{EA}=1 / 1, \mathrm{R}_{\mathrm{f}}=0.44$.

${ }^{1} \mathrm{H}$ NMR (400 MHz, DMSO-d $) \delta 9.68(\mathrm{~s}, 1 \mathrm{H}), 7.29(\mathrm{t}, J=4.2 \mathrm{~Hz}, 1 \mathrm{H}), 7.22(\mathrm{~d}, J=7.3 \mathrm{~Hz}$, 2H), $7.12-7.06(\mathrm{~m}, 2 \mathrm{H}), 6.87(\mathrm{~d}, J=9.0 \mathrm{~Hz}, 2 \mathrm{H}), 6.71(\mathrm{~s}, 1 \mathrm{H}), 4.25(\mathrm{t}, J=9.1 \mathrm{~Hz}, 1 \mathrm{H}), 3.48$ $(\mathrm{s}, 1 \mathrm{H}), 3.07(\mathrm{~s}, 1 \mathrm{H}), 3.00(\mathrm{~d}, J=8.3 \mathrm{~Hz}, 1 \mathrm{H}), 2.31(\mathrm{~d}, J=9.6 \mathrm{~Hz}, 1 \mathrm{H}), 1.85(\mathrm{~d}, J=9.3 \mathrm{~Hz}, 1 \mathrm{H})$, $1.55(\mathrm{~s}, 3 \mathrm{H})$.

${ }^{13} \mathrm{C}$ NMR (101 MHz, DMSO) $\delta 168.45,157.34,150.32,146.80,131.09,129.02,126.58,126.54$, $126.11,122.17,120.91,118.76,114.12,52.67,50.74,46.41,45.93,43.80,23.14$.

HRMS (ESI) (m/z): calcd for $\mathrm{C}_{19} \mathrm{H}_{18} \mathrm{ClNO}_{2}[\mathrm{M}+\mathrm{Na}]^{+}: 350.0924$, found: 350.0924 .

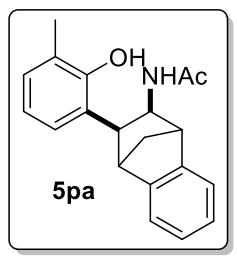

$N$-(3-(2-hydroxy-3-methylphenyl)-1,2,3,4-tetrahydro-1,4-methanonaphthalen-2yl)acetamide (5pa).

This compound was obtained in $53 \%$ yield $(32.6 \mathrm{mg})$ as white solid. Mp: $176-177{ }^{\circ} \mathrm{C}$. Eluent: $\mathrm{PE} / \mathrm{EA}=1 / 1, \mathrm{R}_{\mathrm{f}}=0.49$.

${ }^{1} \mathrm{H}$ NMR $\left(500 \mathrm{MHz}, \mathrm{DMSO}-d_{6}\right) \delta 7.78(\mathrm{~s}, 1 \mathrm{H}), 7.31(\mathrm{dd}, J=5.3,3.1 \mathrm{~Hz}, 1 \mathrm{H}), 7.23(\mathrm{dd}, J=5.3$, $3.1 \mathrm{~Hz}, 1 \mathrm{H}), 7.15-7.06(\mathrm{~m}, 3 \mathrm{H}), 6.98(\mathrm{~d}, J=7.3 \mathrm{~Hz}, 1 \mathrm{H}), 6.89(\mathrm{~d}, J=9.3 \mathrm{~Hz}, 1 \mathrm{H}), 6.82(\mathrm{t}, J$ $=7.5 \mathrm{~Hz}, 1 \mathrm{H}), 4.20(\mathrm{t}, J=8.7 \mathrm{~Hz}, 1 \mathrm{H}), 3.50(\mathrm{~s}, 1 \mathrm{H}), 3.16(\mathrm{~d}, J=5.0 \mathrm{~Hz}, 2 \mathrm{H}), 2.34(\mathrm{~d}, J=9.5$ $\mathrm{Hz}, 1 \mathrm{H}), 2.12$ (s, 3H), $1.88(\mathrm{~d}, J=9.6 \mathrm{~Hz}, 1 \mathrm{H}), 1.52$ (s, 3H).

${ }^{13} \mathrm{C}$ NMR (101 MHz, DMSO) $\delta 168.91,153.34,150.06,146.28,128.50,127.93,126.10,125.58$, 124.57, 121.74, 120.41, 119.56, 52.86, 49.85, 46.56, 45.73, 43.96, 22.43, 16.69.

HRMS (ESI) (m/z): calcd for $\mathrm{C}_{20} \mathrm{H}_{21} \mathrm{NO}_{2}[\mathrm{M}+\mathrm{Na}]^{+}: 330.1470$, found: 330.1466 .

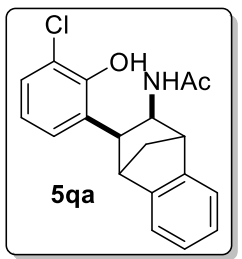

$N$-(3-(3-chloro-2-hydroxyphenyl)-1,2,3,4-tetrahydro-1,4-methanonaphthalen-2yl)acetamide (5qa).

This compound was obtained in $39 \%$ yield $(25.5 \mathrm{mg})$ as white solid. Mp: $172-173{ }^{\circ} \mathrm{C}$. Eluent: $\mathrm{PE} / \mathrm{EA}=1 / 1, \mathrm{R}_{\mathrm{f}}=0.44$. 
${ }^{1} \mathrm{H}$ NMR (400 MHz, DMSO- $\left.d_{6}\right) \delta 8.74(\mathrm{~s}, 1 \mathrm{H}), 7.31(\mathrm{dd}, J=5.5,3.0 \mathrm{~Hz}, 1 \mathrm{H}), 7.28-7.17(\mathrm{~m}$, $3 \mathrm{H}), 7.11(\mathrm{dd}, J=5.3,3.1 \mathrm{~Hz}, 2 \mathrm{H}), 7.00(\mathrm{~d}, J=9.7 \mathrm{~Hz}, 1 \mathrm{H}), 6.90(\mathrm{t}, J=7.8 \mathrm{~Hz}, 1 \mathrm{H}), 4.26(\mathrm{t}, J$ $=8.9 \mathrm{~Hz}, 1 \mathrm{H}), 3.50(\mathrm{~s}, 1 \mathrm{H}), 3.19-3.08(\mathrm{~m}, 2 \mathrm{H}), 2.35(\mathrm{~d}, J=9.7 \mathrm{~Hz}, 1 \mathrm{H}), 1.87(\mathrm{~d}, J=9.7 \mathrm{~Hz}$, $1 \mathrm{H}), 1.53(\mathrm{~s}, 3 \mathrm{H})$.

${ }^{13} \mathrm{C}$ NMR (101 MHz, DMSO) $\delta 169.06,151.52,150.29,146.74,130.85,127.78,126.64,126.52$, $126.18,122.25,120.97,120.82,120.68,53.17,50.50,46.99,46.14,44.73,22.95$.

HRMS (ESI) (m/z): calcd for $\mathrm{C}_{19} \mathrm{H}_{18} \mathrm{ClNO}_{2}[\mathrm{M}+\mathrm{Na}]^{+}: 350.0924$, found: 350.0922 .

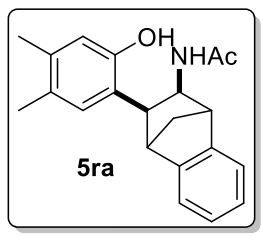

$N$-(3-(2-hydroxy-4,5-dimethylphenyl)-1,2,3,4-tetrahydro-1,4-methanonaphthalen-2yl)acetamide (5ra).

This compound was obtained in $74 \%$ yield $(47.5 \mathrm{mg})$ as white solid. $\mathrm{Mp}: 197-199{ }^{\circ} \mathrm{C}$. Eluent: $\mathrm{PE} / \mathrm{EA}=1 / 1, \mathrm{R}_{\mathrm{f}}=0.35$.

${ }^{1} \mathrm{H}$ NMR (400 MHz, DMSO- $d_{6}$ ) $\delta 8.70(\mathrm{~s}, 1 \mathrm{H}), 7.24$ (ddd, $\left.J=29.4,5.8,3.4 \mathrm{~Hz}, 2 \mathrm{H}\right), 7.08$ (dd, $J=5.2,3.1 \mathrm{~Hz}, 2 \mathrm{H}), 6.97(\mathrm{~s}, 1 \mathrm{H}), 6.69(\mathrm{~d}, J=9.5 \mathrm{~Hz}, 1 \mathrm{H}), 6.51(\mathrm{~s}, 1 \mathrm{H}), 4.16(\mathrm{t}, J=8.9 \mathrm{~Hz}$, 1H), 3.48 (s, 1H), 3.08 (s, 1H), 2.99 (d, $J=8.3 \mathrm{~Hz}, 1 \mathrm{H}), 2.30$ (d, $J=9.5 \mathrm{~Hz}, 1 \mathrm{H}), 2.17$ (s, 3H), $2.11(\mathrm{~s}, 3 \mathrm{H}), 1.86(\mathrm{dd}, J=12.0,9.5 \mathrm{~Hz}, 1 \mathrm{H}), 1.55(\mathrm{~s}, 3 \mathrm{H})$.

${ }^{13} \mathrm{C}$ NMR (101 MHz, DMSO) $\delta 168.64,154.08,150.58,146.87,134.73,128.68,126.49,125.99$, 125.95, 123.93, 122.12, 120.81, 116.17, 52.78, 50.78, 46.59, 46.04, 43.71, 23.29, 19.71, 19.45 . HRMS (ESI) (m/z): calcd for $\mathrm{C}_{21} \mathrm{H}_{23} \mathrm{NO}_{2}[\mathrm{M}+\mathrm{Na}]^{+}: 344.1626$, found: 344.1629 .

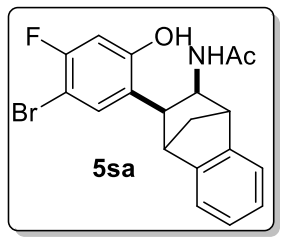

$N$-(3-(5-bromo-4-fluoro-2-hydroxyphenyl)-1,2,3,4-tetrahydro-1,4-methanonaphthalen-2yl)acetamide (5sa).

This compound was obtained in $32 \%$ yield $(24.9 \mathrm{mg})$ as white solid. $\mathrm{Mp}: 106-107{ }^{\circ} \mathrm{C}$. Eluent: $\mathrm{PE} / \mathrm{EA}=1 / 1, \mathrm{R}_{\mathrm{f}}=0.26$.

${ }^{1} \mathrm{H}$ NMR (400 MHz, DMSO- $\left.d_{6}\right) \delta 9.98(\mathrm{~s}, 1 \mathrm{H}), 7.32-7.20(\mathrm{~m}, 3 \mathrm{H}), 7.17-7.00(\mathrm{~m}, 2 \mathrm{H}), 6.53$ $(\mathrm{d}, J=8.4 \mathrm{~Hz}, 1 \mathrm{H}), 4.31(\mathrm{t}, J=9.2 \mathrm{~Hz}, 1 \mathrm{H}), 3.83-3.62(\mathrm{~m}, 1 \mathrm{H}), 3.17-2.98(\mathrm{~m}, 2 \mathrm{H}), 2.45(\mathrm{~s}$, $1 \mathrm{H}), 1.88(\mathrm{~d}, J=9.6 \mathrm{~Hz}, 1 \mathrm{H}), 1.55(\mathrm{~s}, 3 \mathrm{H})$.

${ }^{13} \mathrm{C}$ NMR (101 MHz, DMSO) $\delta 168.09,158.58,157.21,156.16,150.25,145.93,130.44,126.13$, 125.69, 121.76, 120.28, 115.38, 111.32, 52.68, 49.72, 46.82, 45.53, 44.43, 22.51.

${ }^{19} \mathrm{~F}$ NMR (376 MHz, DMSO) $\delta-110.63$.

HRMS (ESI) (m/z): calcd for $\mathrm{C}_{19} \mathrm{H}_{17} \mathrm{BrFNO}_{2}[\mathrm{M}+\mathrm{Na}]^{+}$: 412.0324 , found: 412.0317 . 


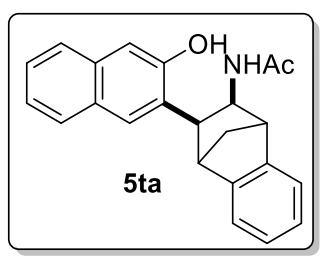

$N$-(3-(3-hydroxynaphthalen-2-yl)-1,2,3,4-tetrahydro-1,4-methanonaphthalen-2yl)acetamide (5ta).

This compound was obtained in $56 \%$ yield $(38.4 \mathrm{mg})$ as yellow solid. Mp: $141-142{ }^{\circ} \mathrm{C}$. Eluent: $\mathrm{PE} / \mathrm{EA}=1 / 1, \mathrm{R}_{\mathrm{f}}=0.27$.

${ }^{1} \mathrm{H}$ NMR (400 MHz, DMSO- $\left.d_{6}\right) \delta 9.65(\mathrm{~s}, 1 \mathrm{H}), 7.84(\mathrm{~d}, J=8.1 \mathrm{~Hz}, 1 \mathrm{H}), 7.77(\mathrm{~s}, 1 \mathrm{H}), 7.62(\mathrm{~d}$, $J=8.2 \mathrm{~Hz}, 1 \mathrm{H}), 7.36-7.24(\mathrm{~m}, 4 \mathrm{H}), 7.12(\mathrm{dd}, J=5.3,3.1 \mathrm{~Hz}, 2 \mathrm{H}), 7.03(\mathrm{~s}, 1 \mathrm{H}), 6.80(\mathrm{~d}, J=$ $10.0 \mathrm{~Hz}, 1 \mathrm{H}), 4.37$ (t, $J=9.2 \mathrm{~Hz}, 1 \mathrm{H}), 3.73(\mathrm{~s}, 1 \mathrm{H}), 3.17$ (d, $J=8.3 \mathrm{~Hz}, 1 \mathrm{H}), 3.09$ (s, 1H), 2.46 $(\mathrm{d}, J=9.6 \mathrm{~Hz}, 1 \mathrm{H}), 1.93(\mathrm{~d}, J=9.6 \mathrm{~Hz}, 1 \mathrm{H}), 1.47(\mathrm{~s}, 3 \mathrm{H})$.

${ }^{13} \mathrm{C}$ NMR (101 MHz, DMSO) $\delta$ 167.99, 154.70, 149.91, 146.49, 133.35, 130.07, 127.68, 126.11, 125.62, 125.33, 125.28, 122.24, 121.68, 120.53, 107.23, 52.28, 50.82, 45.83, 45.45, 44.14, 22.66 .

HRMS (ESI) (m/z): calcd for $\mathrm{C}_{23} \mathrm{H}_{21} \mathrm{NO}_{2}[\mathrm{M}+\mathrm{Na}]^{+}: 366.1470$, found: 366.1465 .

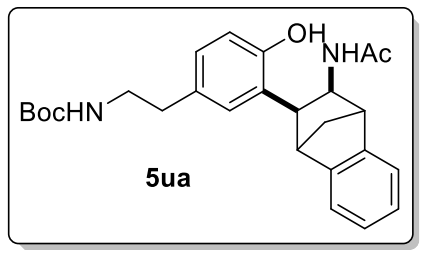

Tert-butyl (3-(3-acetamido-1,2,3,4-tetrahydro-1,4-methanonaphthalen-2-yl)-4hydroxyphenethyl)carbamate (5ua)

This compound was obtained in $72 \%$ yield $(62.8 \mathrm{mg})$ as white solid. $\mathrm{Mp}: 147-148{ }^{\circ} \mathrm{C}$. Eluent: $\mathrm{PE} / \mathrm{EA}=1 / 1, \mathrm{R}_{\mathrm{f}}=0.21$.

${ }^{1} \mathrm{H}$ NMR $\left(400 \mathrm{MHz}, \mathrm{DMSO}-d_{6}\right) \delta 8.91(\mathrm{~s}, 1 \mathrm{H}), 7.29(\mathrm{dd}, J=5.4,3.0 \mathrm{~Hz}, 1 \mathrm{H}), 7.21(\mathrm{dd}, J=5.3$, $3.1 \mathrm{~Hz}, 1 \mathrm{H}), 7.10(\mathrm{dd}, J=5.3,3.1 \mathrm{~Hz}, 2 \mathrm{H}), 7.02(\mathrm{~d}, J=2.1 \mathrm{~Hz}, 1 \mathrm{H}), 6.88-6.78(\mathrm{~m}, 2 \mathrm{H}), 6.61$ $(\mathrm{t}, J=9.5 \mathrm{~Hz}, 2 \mathrm{H}), 4.20(\mathrm{t}, J=8.9 \mathrm{~Hz}, 1 \mathrm{H}), 3.51(\mathrm{~s}, 1 \mathrm{H}), 3.19-3.01(\mathrm{~m}, 5 \mathrm{H}), 2.63(\mathrm{q}, J=6.7$ $\mathrm{Hz}, 3 \mathrm{H}), 2.35(\mathrm{~d}, J=9.5 \mathrm{~Hz}, 1 \mathrm{H}), 1.91-1.81(\mathrm{~m}, 2 \mathrm{H}), 1.52(\mathrm{~s}, 3 \mathrm{H}), 1.39$ (s, 9H).

${ }^{13} \mathrm{C}$ NMR (101 MHz, DMSO) $\delta 168.51,155.98,154.44,150.62,146.83,129.66,127.82,127.58$, $126.63,126.56,126.03,122.19,120.76,114.55,113.25,77.87,52.74,50.63,46.53,46.15$, 43.97, 42.38, 35.54, 28.75, 23.19.

HRMS (ESI) (m/z): calcd for $\mathrm{C}_{26} \mathrm{H}_{32} \mathrm{~N}_{2} \mathrm{O}_{4}[\mathrm{M}+\mathrm{Na}]^{+}:$459.2260, found: 459.2259 .

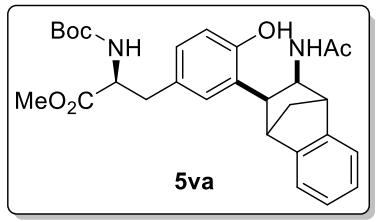

Methyl-3-(3-(3-acetamido-1,2,3,4-tetrahydro-1,4-methanonaphthalen-2-yl)-4hydroxyphenyl)-2-((tert-butoxycarbonyl)amino)propanoate (5va)

This compound was obtained in $69 \%$ yield $(68.2 \mathrm{mg})$ as white solid. Mp: $159-161{ }^{\circ} \mathrm{C}$. Eluent: 
$\mathrm{PE} / \mathrm{EA}=1 / 1, \mathrm{R}_{\mathrm{f}}=0.21$

${ }^{1} \mathrm{H}$ NMR $\left(500 \mathrm{MHz}\right.$, DMSO- $\left.d_{6}\right) \delta 9.02(\mathrm{~d}, J=29.3 \mathrm{~Hz}, 1 \mathrm{H}), 7.40-7.17(\mathrm{~m}, 3 \mathrm{H}), 7.17-6.99$ $(\mathrm{m}, 3 \mathrm{H}), 6.90(\mathrm{t}, J=9.6 \mathrm{~Hz}, 1 \mathrm{H}), 6.62(\mathrm{t}, J=8.4 \mathrm{~Hz}, 1 \mathrm{H}), 6.48(\mathrm{dd}, J=57.8,9.7 \mathrm{~Hz}, 1 \mathrm{H}), 4.29$ $-4.04(\mathrm{~m}, 2 \mathrm{H}), 3.65$ (s, 3H), 3.47 (d, $J=38.9 \mathrm{~Hz}, 1 \mathrm{H}), 3.12$ (qd, $J=20.0,18.8,6.7 \mathrm{~Hz}, 2 \mathrm{H}$ ), 2.95 (ddt, $J=26.3,12.4,6.2 \mathrm{~Hz}, 1 \mathrm{H}$ ), 2.79 (ddd, $J=24.0,13.9,9.9 \mathrm{~Hz}, 1 \mathrm{H}), 2.37$ (dd, $J=27.5$, $9.6 \mathrm{~Hz}, 1 \mathrm{H}), 1.87(\mathrm{~d}, J=9.5 \mathrm{~Hz}, 1 \mathrm{H}), 1.52(\mathrm{~d}, J=4.2 \mathrm{~Hz}, 3 \mathrm{H}), 1.36(\mathrm{~d}, J=3.7 \mathrm{~Hz}, 9 \mathrm{H})$.

${ }^{13} \mathrm{C}$ NMR (101 MHz, DMSO) $\delta 173.46,173.12,168.54,168.42,155.88,155.72,154.77,154.65$, 150.82 , 150.63, 146.84, 146.66, 128.72, 128.45, 128.07, 127.88, 127.74, 127.33, 126.58, $126.05,122.29,120.68,114.61,114.48,78.70,78.58,56.12,55.80,52.77,52.62,52.23,52.19$, 50.53, 50.45, 46.86, 46.66, 46.22, 43.85, 43.60, 36.69, 36.38, 28.62, 28.60, 28.33, 23.21, 23.16. HRMS (ESI) (m/z): calcd for $\mathrm{C}_{28} \mathrm{H}_{34} \mathrm{~N}_{2} \mathrm{O}_{6}[\mathrm{M}+\mathrm{Na}]^{+}:$517.2315, found: 517.2314.

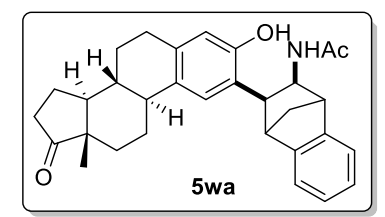

$N$-(3-((8R,9S,13S,14S)-3-hydroxy-13-methyl-17-oxo-7,8,9,11,12,13,14,15,16,17decahydro-6H-cyclopenta[a]phenanthren-2-yl)-1,2,3,4-tetrahydro-1,4methanonaphthalen-2-yl)acetamide (5wa)

This compound was obtained in $64 \%$ yield $(60.1 \mathrm{mg})$ as white solid. Mp: $142-143{ }^{\circ} \mathrm{C}$. Eluent: $\mathrm{PE} / \mathrm{EA}=1 / 1, \mathrm{R}_{\mathrm{f}}=0.24$.

${ }^{1} \mathrm{H}$ NMR (400 MHz, DMSO- $d_{6}$ ) $\delta 8.78(\mathrm{~d}, J=8.0 \mathrm{~Hz}, 1 \mathrm{H}), 7.28(\mathrm{dt}, J=5.2,2.4 \mathrm{~Hz}, 1 \mathrm{H}), 7.19$ $(\mathrm{dd}, J=5.5,3.0 \mathrm{~Hz}, 1 \mathrm{H}), 7.12-7.06(\mathrm{~m}, 3 \mathrm{H}), 6.73(\mathrm{t}, J=10.1 \mathrm{~Hz}, 1 \mathrm{H}), 6.42(\mathrm{~s}, 1 \mathrm{H}), 4.15(\mathrm{dt}$, $J=17.1,8.9 \mathrm{~Hz}, 1 \mathrm{H}), 3.45(\mathrm{~s}, 1 \mathrm{H}), 3.10(\mathrm{~d}, J=14.4 \mathrm{~Hz}, 1 \mathrm{H}), 3.03(\mathrm{t}, J=8.0 \mathrm{~Hz}, 1 \mathrm{H}), 2.74(\mathrm{~d}$, $J=8.0 \mathrm{~Hz}, 2 \mathrm{H}), 2.43(\mathrm{dq}, J=10.7,6.5 \mathrm{~Hz}, 2 \mathrm{H}), 2.31(\mathrm{t}, J=9.3 \mathrm{~Hz}, 1 \mathrm{H}), 2.25-2.17(\mathrm{~m}, 1 \mathrm{H})$, $2.10-2.02(\mathrm{~m}, 1 \mathrm{H}), 1.99-1.90(\mathrm{~m}, 2 \mathrm{H}), 1.87-1.77(\mathrm{~m}, 2 \mathrm{H}), 1.58-1.33(\mathrm{~m}, 9 \mathrm{H}), 0.85(\mathrm{~s}$, $3 \mathrm{H})$.

${ }^{13} \mathrm{C}$ NMR (101 MHz, DMSO) $\delta 219.72,168.10,153.58,153.41,150.19,150.08,146.38,146.32$, $134.45,129.35,126.00,125.49,123.91,123.77,123.68,123.61,121.63,120.26,114.14$, $114.00,52.53,52.30,50.32,50.11,49.63,47.33,46.55,46.32,45.54,43.64,43.38,43.22,38.02$, 37.96, 35.38, 31.37, 28.68, 28.48, 26.32, 26.23, 25.69, 25.48, 22.88, 22.83, 21.14, 13.61, 13.49 . HRMS (ESI) (m/z): calcd for $\mathrm{C}_{31} \mathrm{H}_{35} \mathrm{NO}_{3}[\mathrm{M}+\mathrm{Na}]^{+}$: 492.2515, found: 492.2509 .

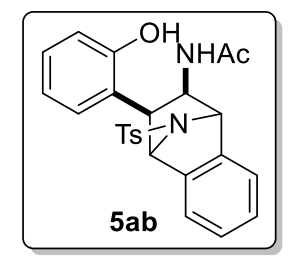

$N$-(3-(2-hydroxyphenyl)-9-tosyl-1,2,3,4-tetrahydro-1,4-epiminonaphthalen-2yl)acetamide (5ab)

This compound was obtained in $71 \%$ yield $(63.6 \mathrm{mg})$ as light gray solid. Mp: $217-219{ }^{\circ} \mathrm{C}$. Eluent: $\mathrm{PE} / \mathrm{EA}=1 / 1, \mathrm{R}_{\mathrm{f}}=0.21$.

${ }^{1} \mathrm{H}$ NMR (400 MHz, DMSO- $\left.d_{6}\right) \delta 9.28(\mathrm{~s}, 1 \mathrm{H}), 7.66(\mathrm{dd}, J=7.7,1.7 \mathrm{~Hz}, 1 \mathrm{H}), 7.33(\mathrm{~d}, J=8.1$ $\mathrm{Hz}, 2 \mathrm{H}), 7.17-7.07$ (m, 2H), 7.00 (d, $J=8.0 \mathrm{~Hz}, 2 \mathrm{H}), 6.97-6.84$ (m, 4H), 6.73 (dd, $J=8.0$, 
$1.2 \mathrm{~Hz}, 1 \mathrm{H}), 6.55(\mathrm{~d}, J=9.3 \mathrm{~Hz}, 1 \mathrm{H}), 5.23(\mathrm{~d}, J=1.5 \mathrm{~Hz}, 1 \mathrm{H}), 4.86(\mathrm{~d}, J=1.4 \mathrm{~Hz}, 1 \mathrm{H}), 4.19$ (t, $J=8.7 \mathrm{~Hz}, 1 \mathrm{H}), 3.27(\mathrm{~d}, J=8.2 \mathrm{~Hz}, 1 \mathrm{H}), 2.21(\mathrm{~s}, 3 \mathrm{H}), 1.55(\mathrm{~s}, 3 \mathrm{H})$.

${ }^{13} \mathrm{C}$ NMR (101 MHz, DMSO) $\delta 169.11,155.87,145.14,143.18,141.22,134.87,129.35,128.32$, 128.24, 128.01, 127.31, 126.87, 124.73, 121.70, 120.73, 119.36, 114.76, 69.77, 67.15, 53.10, 43.74, 22.93, 21.34 .

HRMS (ESI) (m/z): calcd for $\mathrm{C}_{25} \mathrm{H}_{24} \mathrm{~N}_{2} \mathrm{O}_{4} \mathrm{~S}[\mathrm{M}+\mathrm{Na}]^{+}$: 471.1354 , found: 471.1357 .

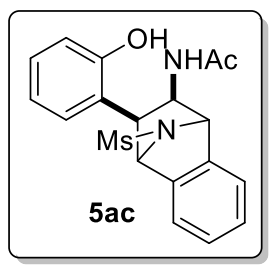

$N$-(3-(2-hydroxyphenyl)-9-(methylsulfonyl)-1,2,3,4-tetrahydro-1,4-epiminonaphthalen-2yl)acetamide (5ac)

This compound was obtained in $63 \%$ yield $(46.9 \mathrm{mg})$ as light gray solid. Mp: $203-204{ }^{\circ} \mathrm{C}$. Eluent: $\mathrm{PE} / \mathrm{EA}=1 / 1, \mathrm{R}_{\mathrm{f}}=0.29$.

${ }^{1} \mathrm{H}$ NMR (400 MHz, DMSO-d $\left.d_{6}\right) \delta 9.62(\mathrm{~s}, 1 \mathrm{H}), 7.80-7.38(\mathrm{~m}, 3 \mathrm{H}), 7.29(\mathrm{~s}, 2 \mathrm{H}), 7.08(\mathrm{t}, J=$ $7.8 \mathrm{~Hz}, 1 \mathrm{H}), 6.85(\mathrm{t}, J=7.5 \mathrm{~Hz}, 1 \mathrm{H}), 6.74(\mathrm{~d}, J=8.0 \mathrm{~Hz}, 1 \mathrm{H}), 6.58(\mathrm{~d}, J=9.2 \mathrm{~Hz}, 1 \mathrm{H}), 5.25(\mathrm{~s}$, $1 \mathrm{H}), 4.83(\mathrm{~s}, 1 \mathrm{H}), 4.29(\mathrm{t}, J=8.7 \mathrm{~Hz}, 1 \mathrm{H}), 3.38(\mathrm{~s}, 1 \mathrm{H}), 2.34(\mathrm{~s}, 3 \mathrm{H}), 1.53(\mathrm{~s}, 3 \mathrm{H}) .{ }^{13} \mathrm{C}$ NMR (101 MHz, DMSO) $\delta 169.05,156.16,146.05,142.45,128.28,128.16,127.91,127.76,124.71$, 122.17, 121.10, 119.12, 114.81, 69.44, 66.97, 52.88, 43.64, 22.91.

HRMS (ESI) (m/z): calcd for $\mathrm{C}_{19} \mathrm{H}_{20} \mathrm{~N}_{2} \mathrm{O}_{4} \mathrm{~S}$ [M+Na] ${ }^{+}: 395.1041$, found: 395.1042 .

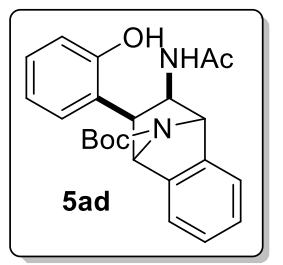

Tert-butyl-2-acetamido-3-(2-hydroxyphenyl)-1,2,3,4-tetrahydro-1,4epiminonaphthalene-9-carboxylate (5ad)

This compound was obtained in $46 \%$ yield $(36.3 \mathrm{mg})$ as white solid. Mp: $167-168{ }^{\circ} \mathrm{C}$. Eluent: $\mathrm{PE} / \mathrm{EA}=1 / 1, \mathrm{R}_{\mathrm{f}}=0.38$.

${ }^{1} \mathrm{H}$ NMR $\left(400 \mathrm{MHz}\right.$, DMSO- $\left.d_{6}\right) \delta 9.20(\mathrm{~s}, 1 \mathrm{H}), 7.53-7.45(\mathrm{~m}, 1 \mathrm{H}), 7.40(\mathrm{~d}, J=8.1 \mathrm{~Hz}, 2 \mathrm{H})$, $7.26-7.18(\mathrm{~m}, 2 \mathrm{H}), 7.06(\mathrm{t}, J=7.8 \mathrm{~Hz}, 1 \mathrm{H}), 6.84(\mathrm{t}, J=7.4 \mathrm{~Hz}, 2 \mathrm{H}), 6.72(\mathrm{~d}, J=7.9 \mathrm{~Hz}, 1 \mathrm{H})$, $5.12(\mathrm{~s}, 1 \mathrm{H}), 4.77(\mathrm{~s}, 1 \mathrm{H}), 4.21(\mathrm{t}, J=8.7 \mathrm{~Hz}, 1 \mathrm{H}), 3.30$ (d, $J=7.5 \mathrm{~Hz}, 1 \mathrm{H}), 1.49$ (s, 3H), 1.28 (s, 9H).

${ }^{13} \mathrm{C}$ NMR (101 MHz, DMSO) $\delta 168.89,155.81,147.47,144.54,131.48,131.04,130.53,128.54$, $128.00,127.91,127.49,127.02,125.56,121.52,120.37,119.26,114.82,80.15,66.13,52.58$, 42.88, 28.36, 27.76, 22.83.

HRMS (ESI) (m/z): calcd for $\mathrm{C}_{23} \mathrm{H}_{26} \mathrm{~N}_{2} \mathrm{O}_{4}[\mathrm{M}+\mathrm{Na}]^{+}: 417.1790$, found: 417.1787. 


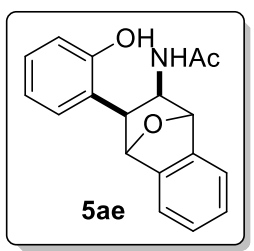

$\mathrm{N}$-(3-(2-hydroxyphenyl)-1,2,3,4-tetrahydro-1,4-epoxynaphthalen-2-yl)acetamide (5ae)

This compound was obtained in $81 \%$ yield $(47.8 \mathrm{mg})$ as white solid. $\mathrm{Mp}: 185-186^{\circ} \mathrm{C}$. Eluent: $\mathrm{PE} / \mathrm{EA}=1 / 1, \mathrm{R}_{\mathrm{f}}=0.29$.

${ }^{1} \mathrm{H}$ NMR $\left(500 \mathrm{MHz}, \mathrm{DMSO}-d_{6}\right) \delta 9.15(\mathrm{~s}, 1 \mathrm{H}), 7.45(\mathrm{dd}, J=5.5,2.8 \mathrm{~Hz}, 1 \mathrm{H}), 7.37$ (ddd, $J=$ 11.6, 6.4, $1.8 \mathrm{~Hz}, 2 \mathrm{H}), 7.26-7.19$ (m, 2H), 7.09 (d, J=9.2 Hz, 1H), 7.04 (td, $J=7.7,1.7 \mathrm{~Hz}$, $1 \mathrm{H}), 6.82(\mathrm{td}, J=7.5,1.3 \mathrm{~Hz}, 1 \mathrm{H}), 6.71(\mathrm{dd}, J=8.0,1.3 \mathrm{~Hz}, 1 \mathrm{H}), 5.45(\mathrm{~s}, 1 \mathrm{H}), 5.20(\mathrm{~s}, 1 \mathrm{H})$, 4.25 (dd, $J=9.2,7.8 \mathrm{~Hz}, 1 \mathrm{H}), 3.45(\mathrm{~d}, J=7.8 \mathrm{~Hz}, 1 \mathrm{H}), 1.44(\mathrm{~s}, 3 \mathrm{H})$.

${ }^{13} \mathrm{C}$ NMR (101 MHz, DMSO) $\delta 168.18,155.27,147.19,143.89,128.62,127.08,127.03,126.53$, $125.29,120.16,118.91,118.63,114.43,83.28,83.23,53.15,42.52,22.21$.

HRMS (ESI) (m/z): calcd for $\mathrm{C}_{18} \mathrm{H}_{17} \mathrm{NO}_{3}[\mathrm{M}+\mathrm{Na}]^{+}: 318.1106$, found: 318.1103 .

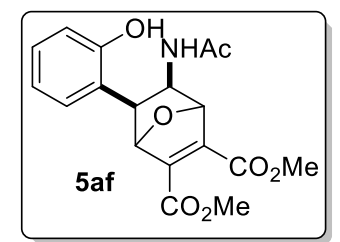

Dimethyl-5-acetamido-6-(2-hydroxyphenyl)-7-oxabicyclo[2.2.1]hept-2-ene-2,3dicarboxylate (5af)

This compound was obtained in $55 \%$ yield $(39.8 \mathrm{mg})$ as white solid. Mp: $158-159{ }^{\circ} \mathrm{C}$. Eluent: $\mathrm{PE} / \mathrm{EA}=1 / 1, \mathrm{R}_{\mathrm{f}}=0.14$.

${ }^{1} \mathrm{H}$ NMR $\left(400 \mathrm{MHz}, \mathrm{DMSO}-d_{6}\right) \delta 9.26(\mathrm{~s}, 1 \mathrm{H}), 7.19(\mathrm{~d}, J=7.6 \mathrm{~Hz}, 1 \mathrm{H}), 7.10(\mathrm{~d}, J=9.3 \mathrm{~Hz}$, 1H), $7.06-6.98(\mathrm{~m}, 1 \mathrm{H}), 6.77(\mathrm{t}, J=7.5 \mathrm{~Hz}, 1 \mathrm{H}), 6.71(\mathrm{~d}, J=8.0 \mathrm{~Hz}, 1 \mathrm{H}), 5.28(\mathrm{~s}, 1 \mathrm{H}), 5.02$ (s, 1H), $4.50-4.33(\mathrm{~m}, 1 \mathrm{H}), 3.76(\mathrm{~d}, J=10.9 \mathrm{~Hz}, 6 \mathrm{H}), 3.59$ (d, $J=7.7 \mathrm{~Hz}, 1 \mathrm{H}), 1.40(\mathrm{~s}, 3 \mathrm{H})$. ${ }^{13} \mathrm{C} \mathrm{NMR}(101 \mathrm{MHz}, \mathrm{DMSO}) \delta 168.78,162.94,162.78,155.98,146.83,143.03,128.81,127.81$, 124.64, 119.05, 114.90, 85.08, 85.06, 52.87, 51.24, 22.61.

HRMS (ESI) (m/z): calcd for $\mathrm{C}_{18} \mathrm{H}_{19} \mathrm{NO}_{7}[\mathrm{M}+\mathrm{Na}]^{+}: 384.1059$, found: 384.1061 .

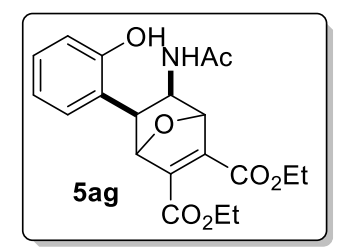

Diethyl-5-acetamido-6-(2-hydroxyphenyl)-7-oxabicyclo[2.2.1]hept-2-ene-2,3dicarboxylate (5ag)

This compound was obtained in $68 \%$ yield $(52.9 \mathrm{mg})$ as white solid. $\mathrm{Mp}: 199-201{ }^{\circ} \mathrm{C}$. Eluent: $\mathrm{PE} / \mathrm{EA}=1 / 1, \mathrm{R}_{\mathrm{f}}=0.21$.

${ }^{1} \mathrm{H}$ NMR (400 MHz, DMSO- $\left.d_{6}\right) \delta 9.50(\mathrm{~s}, 1 \mathrm{H}), 7.25-7.11(\mathrm{~m}, 2 \mathrm{H}), 7.11-6.97(\mathrm{~m}, 1 \mathrm{H}), 6.90$ $-6.71(\mathrm{~m}, 2 \mathrm{H}), 5.24(\mathrm{~s}, 1 \mathrm{H}), 5.11-4.96(\mathrm{~m}, 1 \mathrm{H}), 4.45-4.35(\mathrm{~m}, 1 \mathrm{H}), 4.23(\mathrm{dq}, J=9.5,7.1$ $\mathrm{Hz}, 4 \mathrm{H}), 3.59$ (d, $J=7.7 \mathrm{~Hz}, 1 \mathrm{H}), 1.42(\mathrm{~s}, 3 \mathrm{H}), 1.27-1.20$ (m, 6H). 
${ }^{13} \mathrm{C}$ NMR (101 MHz, DMSO) $\delta 168.37,162.17,162.02,155.61,146.06,142.19,128.33,127.23$, 124.02, 118.42, 114.49, 84.74, 84.47, 61.22, 50.86, 22.13, 13.87 .

HRMS (ESI) (m/z): calcd for $\mathrm{C}_{20} \mathrm{H}_{23} \mathrm{NO}_{7}[\mathrm{M}+\mathrm{Na}]^{+}: 412.1372$, found: 412.1371 .

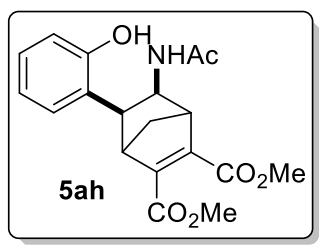

Dimethyl -5-acetamido-6-(2-hydroxyphenyl)bicyclo[2.2.1]hept-2-ene-2,3-dicarboxylate (5ah)

This compound was obtained in $77 \%$ yield $(55.3 \mathrm{mg})$ as white solid. $\mathrm{Mp}: 204-205^{\circ} \mathrm{C}$. Eluent: $\mathrm{PE} / \mathrm{EA}=1 / 1, \mathrm{R}_{\mathrm{f}}=0.17$.

${ }^{1} \mathrm{H}$ NMR (400 MHz, DMSO- $\left.d_{6}\right) \delta 9.27(\mathrm{~s}, 1 \mathrm{H}), 7.21-6.97(\mathrm{~m}, 2 \mathrm{H}), 6.76(\mathrm{dt}, J=28.9,8.4 \mathrm{~Hz}$, $3 \mathrm{H}), 4.43(\mathrm{t}, J=9.1 \mathrm{~Hz}, 1 \mathrm{H}), 3.74(\mathrm{~s}, 3 \mathrm{H}), 3.70(\mathrm{~s}, 3 \mathrm{H}), 3.21(\mathrm{~d}, J=8.4 \mathrm{~Hz}, 1 \mathrm{H}), 2.91(\mathrm{~s}, 1 \mathrm{H})$, $2.15(\mathrm{~d}, J=9.8 \mathrm{~Hz}, 1 \mathrm{H}), 1.85(\mathrm{~s}, 1 \mathrm{H}), 1.49(\mathrm{~s}, 3 \mathrm{H})$.

${ }^{13} \mathrm{C}$ NMR (101 MHz, DMSO) $\delta 168.52,164.84,156.38,147.91,144.22,127.69,127.27,126.11$, 119.09, 114.58, 52.50, 52.47, 51.95, 50.45, 47.87, 44.95, 41.24, 23.10 .

HRMS (ESI) (m/z): calcd for $\mathrm{C}_{19} \mathrm{H}_{21} \mathrm{NO}_{6}[\mathrm{M}+\mathrm{Na}]^{+}$: 382.1267, found: 382.1264 .

\section{Mechanistic Studies}

Ortho deuteration experiments:

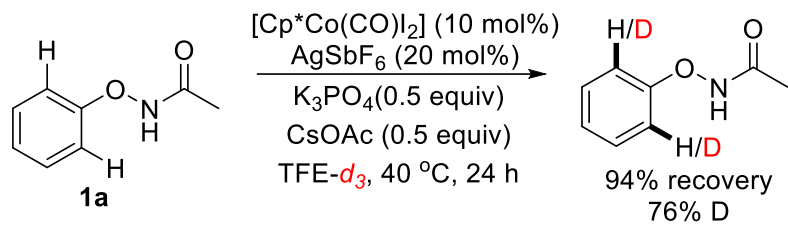

A $10 \mathrm{~mL}$ oven-dried Schlenk tube equipped with a magnetic stir bar was dried under vacuum. 1a (0.20 mmol, 1.0 equiv) and the $\left[\mathrm{Cp} * \mathrm{Co}(\mathrm{CO}) \mathrm{I}_{2}\right]$-catalyst $(9.5 \mathrm{mg}, 10 \mathrm{~mol} \%)$ were added to the tube. Subsequently, the reaction tube was charged with $\mathrm{AgSbF}_{6}(13.7 \mathrm{mg}, 20 \mathrm{~mol} \%), \mathrm{K}_{3} \mathrm{PO}_{4}$ (21.2 mg, 0.5 equiv) and $\mathrm{CsOAc}$ (19.2 $\mathrm{mg}, 0.5$ equiv), in the glovebox. TFE- $d_{3}(1.0 \mathrm{~mL}, 0.2 \mathrm{M})$ was added via syringe. The Schlenk tube was sealed and stirred for $24 \mathrm{~h}$ at $40{ }^{\circ} \mathrm{C}$ in an oil bath. The crude reaction mixture was diluted with dichloromethane $(5 \mathrm{~mL})$ transferred to a $100 \mathrm{~mL}$ round bottom flask, adsorbed on silica and purified by silica gel flash chromatography (PE/ EA).

${ }^{1} \mathrm{H}-\mathrm{NMR}$ of recovered $\mathbf{1 a}$ 


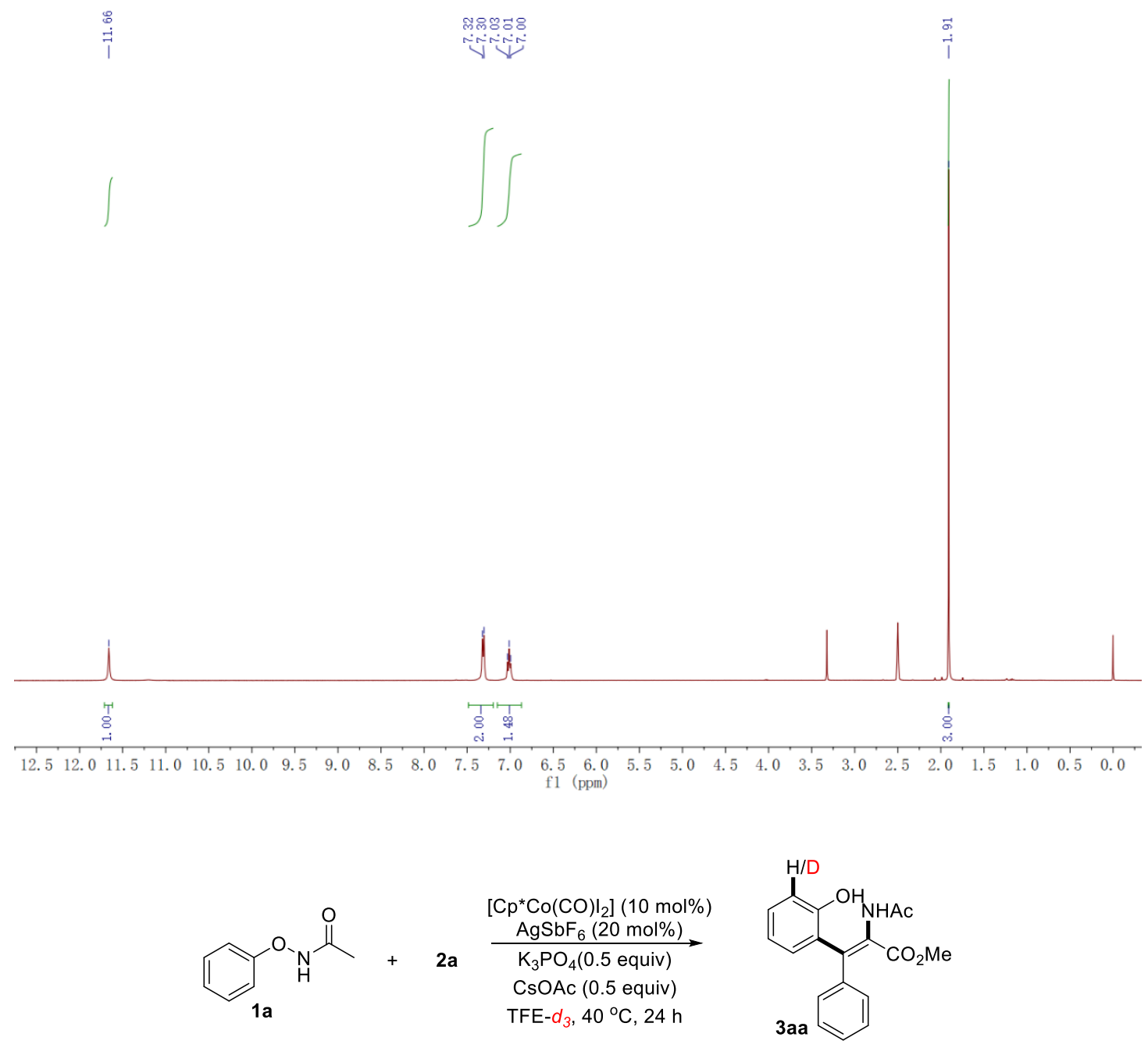

A $10 \mathrm{~mL}$ oven-dried Schlenk tube equipped with a magnetic stir bar was dried under vacuum. 1a $\left(0.20 \mathrm{mmol}, 1.0\right.$ equiv) and the $\left[\mathrm{Cp} * \mathrm{Co}(\mathrm{CO}) \mathrm{I}_{2}\right]$-catalyst $(9.5 \mathrm{mg}, 10 \mathrm{~mol} \%)$ were added to the tube. Subsequently, the reaction tube was charged with AgSbF6 (13.7 mg, $20 \mathrm{~mol} \%$ ), $\mathrm{K}_{3} \mathrm{PO}_{4}$ (21.2 $\mathrm{mg}, 50 \mathrm{~mol} \%$ ) and CsOAc (19.2 $\mathrm{mg}, 50 \mathrm{~mol} \%)$, in the glovebox. TFE- $d_{3}(1.0 \mathrm{~mL}, 0.2 \mathrm{M})$ was added via syringe, followed by propiolate $\mathbf{2 a}(0.22 \mathrm{mmol}, 1.1$ equiv) via syringe. The Schlenk tube was sealed and stirred for $24 \mathrm{~h}$ at $40^{\circ} \mathrm{C}$ in an oil bath. The crude reaction mixture was diluted with dichloromethane $(5 \mathrm{~mL})$ transferred to a $100 \mathrm{~mL}$ round bottom flask, adsorbed on silica and purified by silica gel flash chromatography (PE/ EA).

${ }^{1} \mathrm{H}-\mathrm{NMR}$ of recovered 3aa 


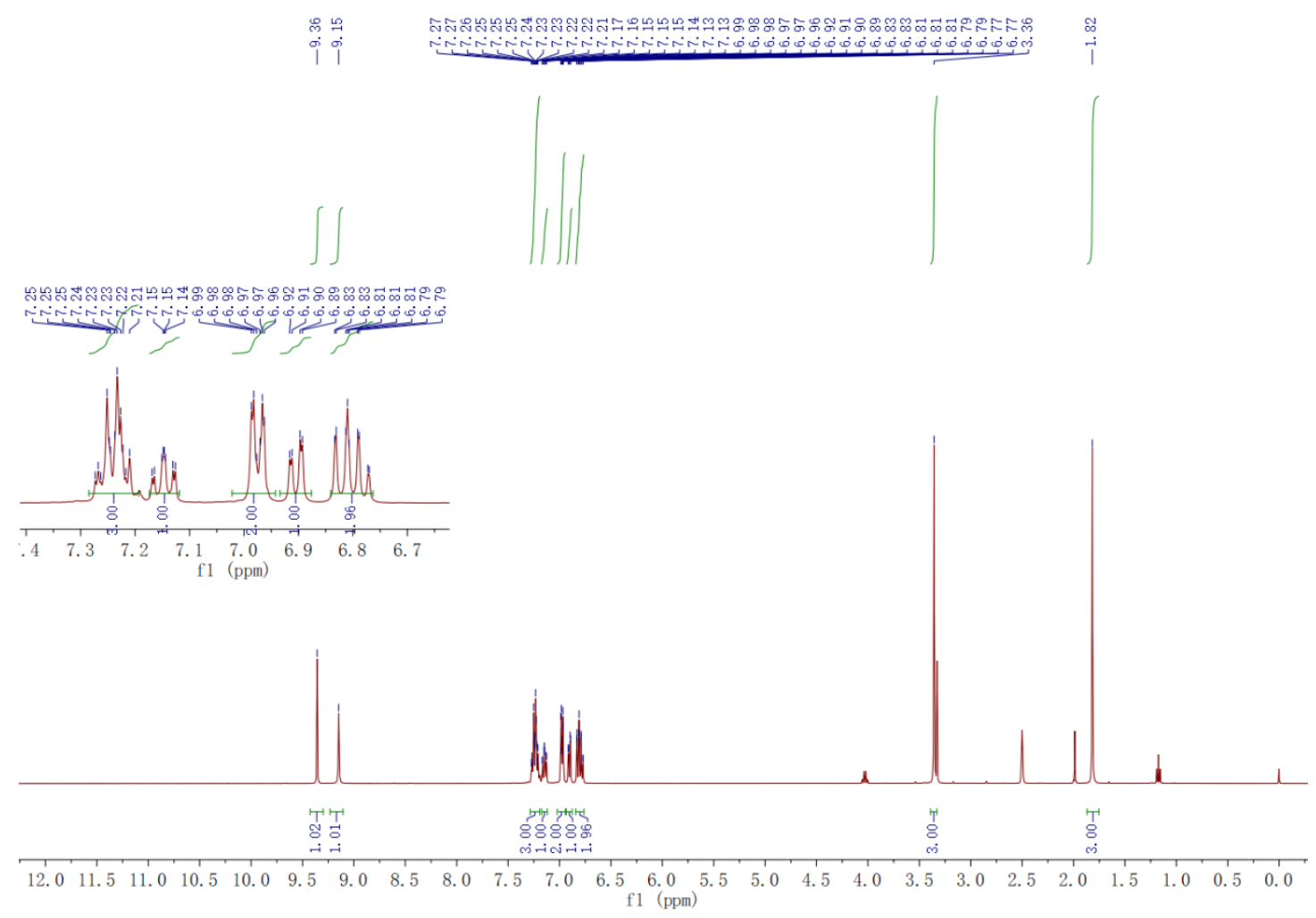

\section{Competition experiment:}

In oven-dried tube equipped with a magnetic stir bar was dried under vacuum, 1a $(1.0 \mathrm{mmol}$, 1.0 equiv) and $\mathbf{1 a - d} 5(0.1 \mathrm{mmol}, 1.0$ equiv $)$ were allowed to react with propiolate $\mathbf{2 a}(0.22 \mathrm{mmol}$, 1.1 equiv) under standard reaction conditions. The reaction was allowed to stir at $40{ }^{\circ} \mathrm{C}$ for $1 \mathrm{~h}$. Afterwards, the solvent was concentrated in vacuum and the residue was purified by silica gel flash chromatography to provide the target mixture of 3aa and 3aa-d4 as a white solid (8.1 mg, $13 \%$ yield $)$. The value of $\mathrm{KIE}\left(\mathrm{k}_{\mathrm{H}} / \mathrm{k}_{\mathrm{D}}=2.45\right)$ was obtained based on ${ }^{1} \mathrm{H}$ NMR. 


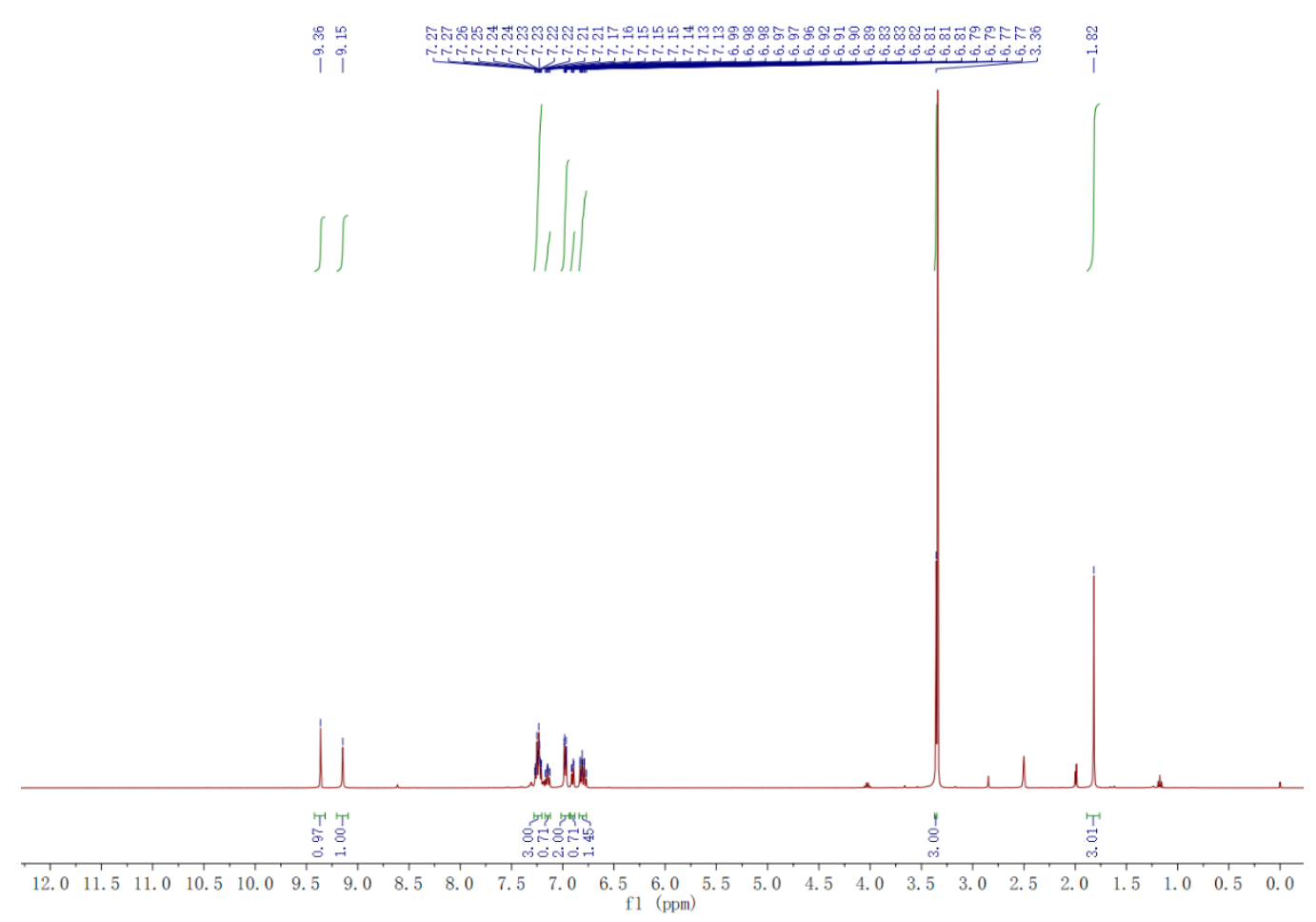

\section{Parallel experiment:}

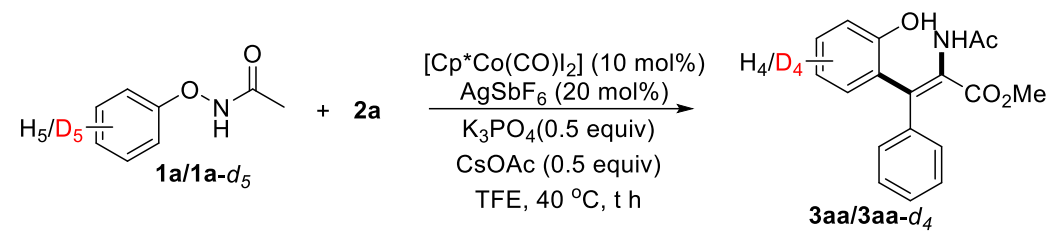

In oven-dried tube equipped with a magnetic stir bar was dried under vacuum. 1a $(0.2 \mathrm{mmol}$, 1.0 equiv) or 1a-d5 ( $0.2 \mathrm{mmol}, 1.0$ equiv) was allowed to react with propiolate $\mathbf{2 a}(0.22 \mathrm{mmol}$, 1.1 equiv) under standard reaction conditions, respectively. The mixture was allowed to stir at $40{ }^{\circ} \mathrm{C}$ for the indicated time. The samples were carried out at 1, 2, 3 and $4 \mathrm{~h}$.

Table S3. Kinetic experiments of $\mathbf{1 a}$

\begin{tabular}{ccc}
\hline Entry & Reaction time $(\mathrm{h})$ & Isolated yield $(\%)$ \\
\hline 1 & 1 & 5.5 \\
2 & 2 & 13.5 \\
3 & 3 & 28.5 \\
4 & 4 & 36.5 \\
\hline
\end{tabular}




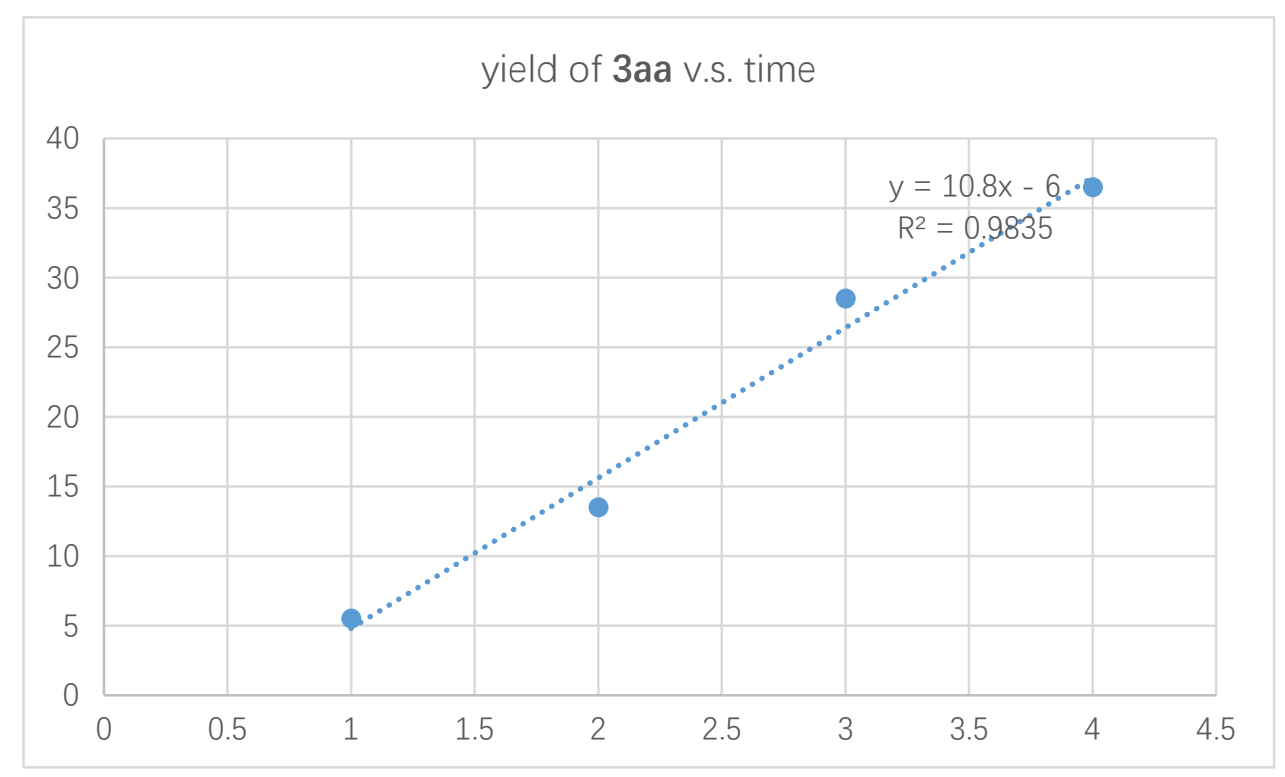

Table S4. Kinect experiments of 1a-d5

\begin{tabular}{ccc}
\hline Entry & Reaction time $(\mathrm{h})$ & Isolated yield $(\%)$ \\
\hline 1 & 1 & 6.5 \\
2 & 2 & 13.5 \\
3 & 3 & 19 \\
4 & 4 & 27 \\
\hline
\end{tabular}

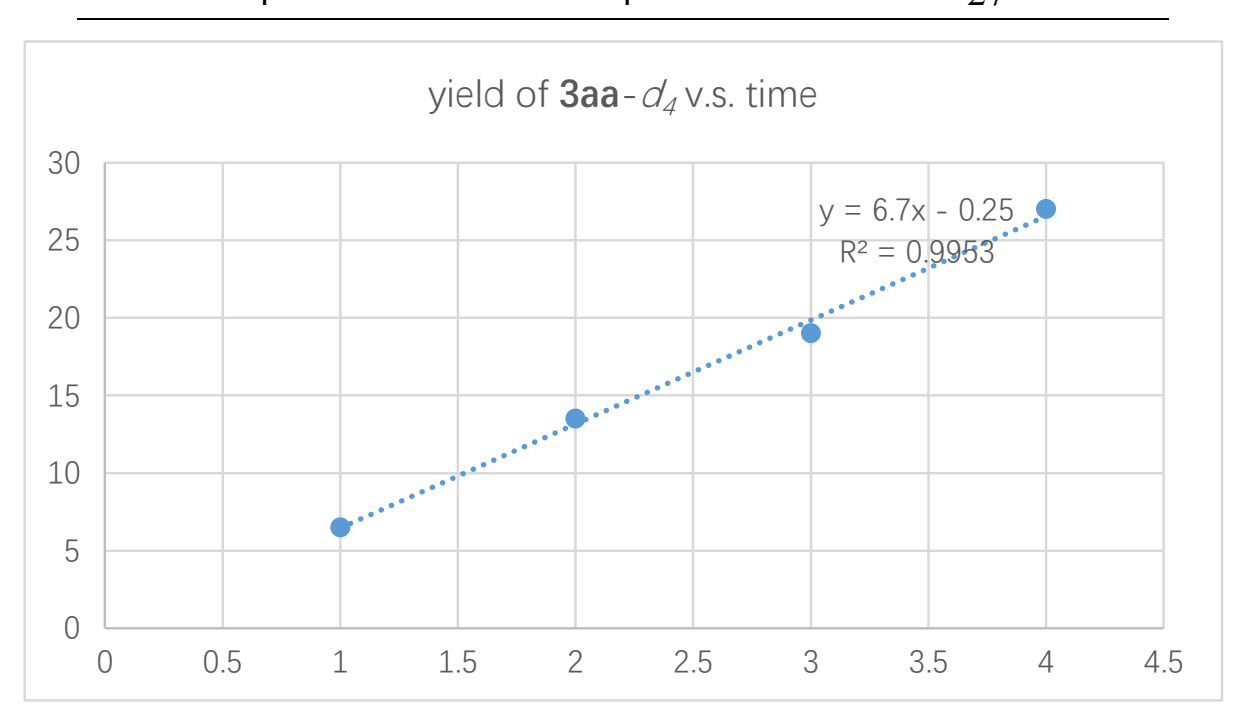

Thus, $\mathrm{k}_{\mathrm{H}} / \mathrm{k}_{\mathrm{D}}=10.8 / 6.7=1.61$.

${ }^{1} \mathrm{H}-\mathrm{NMR}$ of recovered $\mathbf{3 a a}-d_{4}$ 


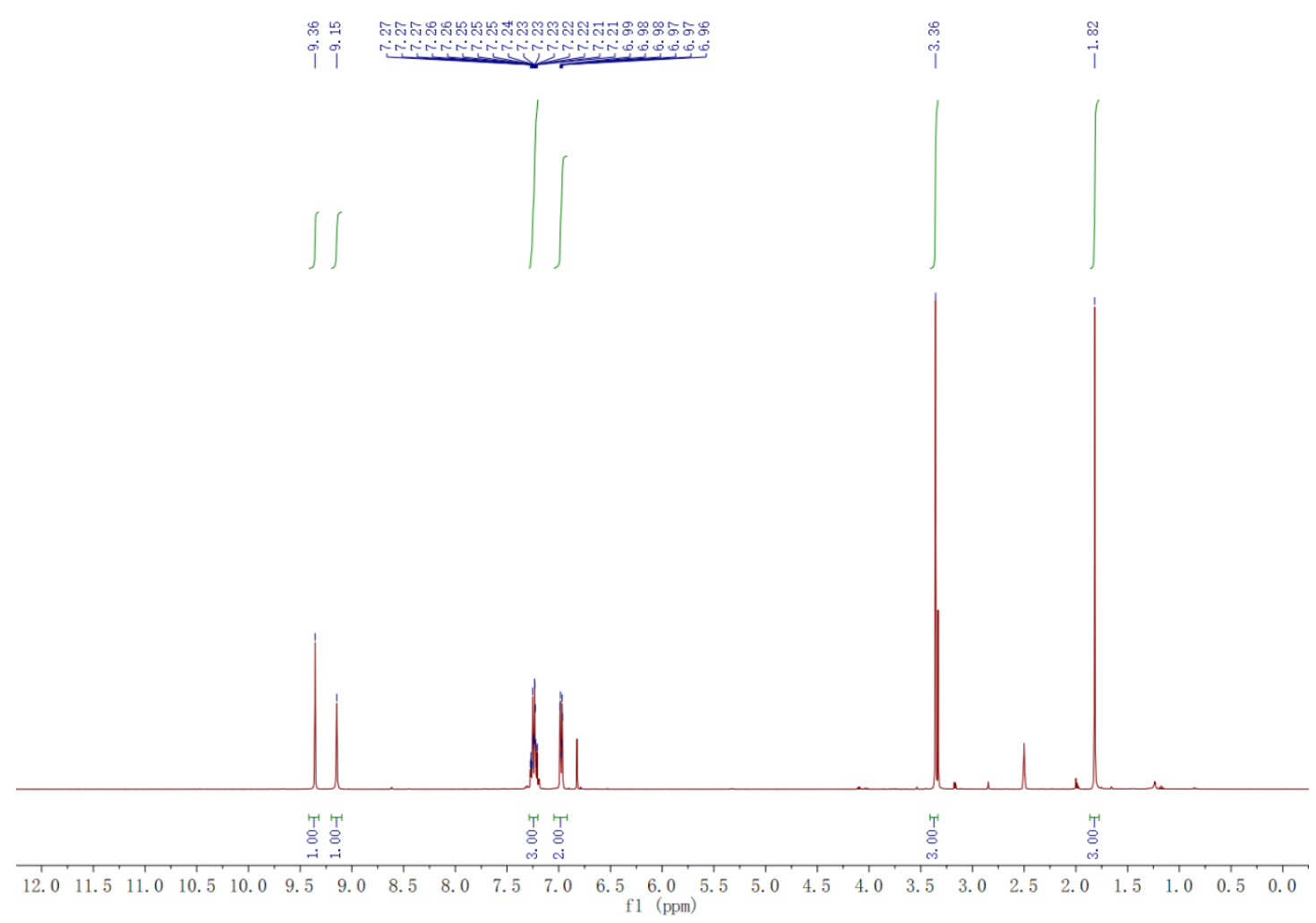

6. Crystallographic data 3am, 3ao, and 5ta

\section{Crystallographic data 3am}

Table S5 Crystal data and structure refinement for 3am.

Identification code 3am

Empirical formula

$\mathrm{C}_{15} \mathrm{H}_{11} \mathrm{NO}_{3} \mathrm{~S}$

Formula weight

285.31

Temperature/K

298.15

Crystal system

monoclinic

Space group

$\mathrm{P} 2{ }_{1} / \mathrm{c}$

$\mathrm{a} / \AA ̊ \AA$

9.5216(9)

$\mathrm{b} / \AA$

$18.5806(16)$

$\mathrm{c} / \AA$

$14.8497(15)$

$\alpha /{ }^{\circ}$

90.00

$\beta /{ }^{\circ}$

96.516(2)

$\gamma /{ }^{\circ}$

90.00

Volume/ $\AA^{3}$

2610.2(4)

Z

8

$\rho_{\text {calc }} \mathrm{g} / \mathrm{cm}^{3}$

1.452

$\mu / \mathrm{mm}^{-1}$

0.254 


\begin{tabular}{|c|c|}
\hline $\mathrm{F}(000)$ & 1184.0 \\
\hline Crystal size $/ \mathrm{mm}^{3}$ & $0.45 \times 0.13 \times 0.1$ \\
\hline Radiation & $\operatorname{MoK} \alpha(\lambda=0.71073)$ \\
\hline \multicolumn{2}{|c|}{$2 \Theta$ range for data collection $/{ }^{\circ} 4.38$ to 50.04} \\
\hline Index ranges & $-11 \leq \mathrm{h} \leq 11,-22 \leq \mathrm{k} \leq 22,-3 \leq 1 \leq 17$ \\
\hline Reflections collected & 4608 \\
\hline Independent reflections & $4608\left[R_{\text {int }}=0.0000, R_{\text {sigma }}=0.1011\right]$ \\
\hline Data/restraints/parameters & $4608 / 18 / 366$ \\
\hline Goodness-of-fit on $\mathrm{F}^{2}$ & 1.093 \\
\hline Final R indexes $[\mathrm{I}>=2 \sigma(\mathrm{I})]$ & $\mathrm{R}_{1}=0.0809, \mathrm{wR}_{2}=0.2044$ \\
\hline Final $R$ indexes [all data] & $\mathrm{R}_{1}=0.1474, \mathrm{wR}_{2}=0.2380$ \\
\hline Largest diff. peak/hole / e $\AA$ & $30.47 /-0.26$ \\
\hline
\end{tabular}

Table S6 Fractional Atomic Coordinates $\left(\times 10^{4}\right)$ and Equivalent Isotropic Displacement Parameters $\left(\AA^{2} \times 10^{3}\right)$ for $3 \mathrm{am} . \mathrm{U}_{\text {eq }}$ is defined as $1 / 3$ of of the trace of the orthogonalised $U_{I J}$ tensor.

\begin{tabular}{lrrrr} 
Atom & \multicolumn{2}{r}{$\boldsymbol{U}$} & $\mathbf{U}(\mathbf{e q})$ \\
$\mathrm{N} 1$ & $6965(4)$ & $8810(2)$ & $3391(3)$ & $38.7(11)$ \\
$\mathrm{N} 2$ & $2020(5)$ & $8816(2)$ & $3401(3)$ & $40.5(12)$ \\
O1 & $6007(4)$ & $7777(2)$ & $1326(3)$ & $55.9(12)$ \\
O2 & $7581(2)$ & $2505(3)$ & $73.7(15)$ \\
O3 & $8648(2)$ & $4101(3)$ & $56.7(12)$ \\
O4 & $5026(4)$ & $7678.2(19)$ & $1432(3)$ & $52.7(11)$ \\
O5 & $1111(4)$ & $7571(2)$ & $2600(3)$ & $66.0(13)$ \\
O6 & $2735(5)$ & $8938(3)$ & $4003(3)$ & $66.5(13)$ \\
S1 & $-30(4)$ & $10191.5(9)$ & $3375.9(12)$ & $45.5(5)$ \\
S2 & $10387.7(10)$ & $2521.5(14)$ & $54.9(6)$ \\
S1 & $15181(2)$ & $10439(5)$ & $1448(6)$ & $45.5(5)$ \\
S2 & $10095(5)$ & $2328(13)$ & $54.9(6)$ \\
C1 & $5189(18)$ & $7979(3)$ & $2151(4)$ & $47.7(15)$ \\
C2 & $-1435(8)$ & $8657(3)$ & $2523(4)$ & $38.9(14)$ \\
C3 & $6682(6)$ & $9113(3)$ & $2047(3)$ & $32.5(12)$ \\
C4 & $6260(5)$ & $8834(3)$ & $1223(3)$ & $34.7(13)$ \\
C5 & $5340(5)$ & $8177(3)$ & $892(4)$ & $39.5(14)$ \\
C6 & $4540(5)$ & $7878(3)$ & $122(4)$ & $54.0(17)$ \\
C7 & $4909(6)$ & $8234(3)$ & $-326(4)$ & $55.9(18)$ \\
C8 & $4209(7)$ & $8872(3)$ & $-2(4)$ & $48.3(16)$ \\
& $3081(7)$ & & &
\end{tabular}




\begin{tabular}{|c|c|c|c|c|}
\hline C9 & $3361(6)$ & $9179(3)$ & $759(4)$ & $40.6(14)$ \\
\hline $\mathrm{C} 10$ & $5162(5)$ & $9867(2)$ & 2302(3) & $34.1(13)$ \\
\hline $\mathrm{C} 11$ & $5098(10)$ & $10458(3)$ & $1722(5)$ & $45.5(5)$ \\
\hline C11' & $5050(70)$ & $10294(6)$ & $3062(14)$ & $45.5(5)$ \\
\hline $\mathrm{C} 12$ & $5059(6)$ & $11122(3)$ & 2131(4) & $52.9(16)$ \\
\hline $\mathrm{C} 13$ & $5081(6)$ & 11044(3) & $3035(4)$ & $55.0(17)$ \\
\hline $\mathrm{C} 14$ & 6292(6) & $8754(3)$ & 4141(4) & $40.0(14)$ \\
\hline $\mathrm{C} 15$ & $7183(6)$ & $8842(3)$ & $5029(4)$ & $54.6(17)$ \\
\hline $\mathrm{C} 16$ & $1815(6)$ & 7934(3) & $2218(4)$ & $47.1(15)$ \\
\hline $\mathrm{C} 17$ & $1376(5)$ & $8632(3)$ & 2531(4) & $35.1(13)$ \\
\hline $\mathrm{C} 18$ & $441(5)$ & $9058(3)$ & 2033(4) & $34.9(13)$ \\
\hline C19 & $-266(6)$ & $8758(3)$ & 1190(4) & $42.1(14)$ \\
\hline $\mathrm{C} 20$ & $85(6)$ & $8076(3)$ & $934(4)$ & $44.0(15)$ \\
\hline $\mathrm{C} 21$ & $-521(7)$ & $7759(3)$ & $144(4)$ & $62.7(19)$ \\
\hline $\mathrm{C} 22$ & $-1504(9)$ & $8134(4)$ & $-411(5)$ & $80(2)$ \\
\hline $\mathrm{C} 23$ & $-1873(8)$ & $8807(4)$ & $-179(5)$ & $80(2)$ \\
\hline $\mathrm{C} 24$ & $-1258(7)$ & 9131(4) & $601(4)$ & $65(2)$ \\
\hline $\mathrm{C} 25$ & $172(5)$ & 9806(3) & 2284(3) & $37.3(13)$ \\
\hline $\mathrm{C} 26$ & $-1081(8)$ & 10184(4) & $2356(8)$ & $54.9(6)$ \\
\hline C26' & $950(30)$ & 10439(9) & $2320(40)$ & $54.9(6)$ \\
\hline $\mathrm{C} 27$ & $-914(7)$ & 10903(3) & 2587(4) & $56.1(17)$ \\
\hline $\mathrm{C} 28$ & $463(6)$ & $11080(3)$ & 2667(4) & $60.2(18)$ \\
\hline $\mathrm{C} 29$ & $1264(6)$ & $8938(3)$ & 4096(4) & $45.3(15)$ \\
\hline $\mathrm{C} 30$ & 2088(7) & $9069(4)$ & 4997(4) & $65.0(19)$ \\
\hline
\end{tabular}

Table S7 Anisotropic Displacement Parameters $\left(\AA^{2} \times 10^{3}\right)$ for 3am. The Anisotropic displacement factor exponent takes the form: $-2 \pi^{2}\left[h^{2} a^{* 2} U_{11}+2 h k a * b * U_{12}+\ldots\right]$.

\begin{tabular}{lrrrrrr} 
Atom & \multicolumn{1}{l}{$\mathbf{U}_{\mathbf{1 1}}$} & \multicolumn{1}{l}{$\mathbf{U}_{\mathbf{2 2}}$} & \multicolumn{1}{l}{$\mathbf{U}_{\mathbf{3 3}}$} & \multicolumn{1}{c}{$\mathbf{U}_{\mathbf{2 3}}$} & \multicolumn{1}{c}{$\mathbf{U}_{\mathbf{1 2}}$} \\
$\mathrm{N} 1$ & $29(2)$ & $52(3)$ & $34(3)$ & $4(2)$ & $1(2)$ & $-3(2)$ \\
$\mathrm{N} 2$ & $25(2)$ & $54(3)$ & $41(3)$ & $0(2)$ & $-3(2)$ & $-3(2)$ \\
$\mathrm{O} 1$ & $69(3)$ & $41(2)$ & $57(3)$ & $-10(2)$ & $4(2)$ & $14(2)$ \\
$\mathrm{O} 2$ & $89(4)$ & $57(3)$ & $71(3)$ & $1(2)$ & $-4(3)$ & $37(3)$ \\
O3 & $36(2)$ & $74(3)$ & $60(3)$ & $21(2)$ & $5(2)$ & $-2(2)$ \\
O4 & $64(3)$ & $40(2)$ & $53(3)$ & $-4(2)$ & $1(2)$ & $5(2)$ \\
O5 & $77(3)$ & $51(3)$ & $67(3)$ & $5(2)$ & $-5(2)$ & $24(2)$ \\
O6 & $35(3)$ & $113(4)$ & $51(3)$ & $7(3)$ & $3(2)$ & $-2(2)$ \\
S1 & $59.3(12)$ & $41.2(9)$ & $35.3(10)$ & $-5.1(8)$ & $2.1(10)$ & $4.6(8)$ \\
S2 & $36.3(11)$ & $47.9(10)$ & $79.5(14)$ & $-7.0(9)$ & $2.1(9)$ & $-5.9(8)$ \\
S1 & $59.3(12)$ & $41.2(9)$ & $35.3(10)$ & $-5.1(8)$ & $2.1(10)$ & $4.6(8)$ \\
& & & & & & 39
\end{tabular}




\begin{tabular}{|c|c|c|c|c|c|c|}
\hline S2' & $36.3(11)$ & $47.9(10)$ & $79.5(14)$ & $-7.0(9)$ & $2.1(9)$ & $-5.9(8)$ \\
\hline $\mathrm{C} 1$ & $54(4)$ & $40(4)$ & $49(4)$ & $-2(3)$ & $6(3)$ & $4(3)$ \\
\hline $\mathrm{C} 2$ & $38(3)$ & $40(3)$ & $40(3)$ & $-1(3)$ & $7(3)$ & $-2(2)$ \\
\hline $\mathrm{C} 3$ & $32(3)$ & $35(3)$ & $31(3)$ & $1(2)$ & $5(2)$ & $0(2)$ \\
\hline $\mathrm{C} 4$ & $39(3)$ & $34(3)$ & $33(3)$ & $2(2)$ & $10(2)$ & $-2(2)$ \\
\hline $\mathrm{C} 5$ & $50(4)$ & $32(3)$ & $37(3)$ & $-2(3)$ & $8(3)$ & 1(3) \\
\hline C6 & $70(5)$ & $46(4)$ & $47(4)$ & $-14(3)$ & $12(3)$ & $-4(3)$ \\
\hline $\mathrm{C} 7$ & $69(5)$ & $62(4)$ & $36(4)$ & $-9(3)$ & 1(3) & $-14(3)$ \\
\hline $\mathrm{C} 8$ & $52(4)$ & $52(4)$ & $39(4)$ & $2(3)$ & $-5(3)$ & $-3(3)$ \\
\hline C9 & $45(4)$ & $40(3)$ & $38(3)$ & $-1(3)$ & $10(3)$ & $-2(3)$ \\
\hline $\mathrm{C} 10$ & $27(3)$ & $39(3)$ & $36(3)$ & $-5(3)$ & $4(2)$ & $2(2)$ \\
\hline $\mathrm{C} 11$ & $59.3(12)$ & 41.2(9) & $35.3(10)$ & $-5.1(8)$ & $2.1(10)$ & $4.6(8)$ \\
\hline $\mathrm{C} 11^{\prime}$ & $59.3(12)$ & 41.2(9) & $35.3(10)$ & $-5.1(8)$ & $2.1(10)$ & $4.6(8)$ \\
\hline $\mathrm{C} 12$ & $57(4)$ & $38(4)$ & $66(5)$ & $7(3)$ & $18(3)$ & $-1(3)$ \\
\hline $\mathrm{C} 13$ & $55(4)$ & $45(4)$ & $64(5)$ & $-16(3)$ & $6(3)$ & $-3(3)$ \\
\hline $\mathrm{C} 14$ & $34(3)$ & $43(3)$ & $44(4)$ & 11(3) & $4(3)$ & $6(3)$ \\
\hline $\mathrm{C} 15$ & $43(4)$ & $81(4)$ & $39(4)$ & $3(3)$ & $5(3)$ & $4(3)$ \\
\hline $\mathrm{C} 16$ & $53(4)$ & $42(4)$ & $46(4)$ & $3(3)$ & $5(3)$ & $-1(3)$ \\
\hline $\mathrm{C} 17$ & $32(3)$ & $35(3)$ & $39(3)$ & $1(2)$ & $6(2)$ & $-3(2)$ \\
\hline $\mathrm{C} 18$ & $31(3)$ & $34(3)$ & $40(3)$ & $5(2)$ & $4(2)$ & $-3(2)$ \\
\hline C19 & $49(4)$ & $37(3)$ & $38(3)$ & $0(3)$ & $-2(3)$ & $-7(3)$ \\
\hline $\mathrm{C} 20$ & $50(4)$ & $36(3)$ & $46(4)$ & $3(3)$ & 2(3) & $-3(3)$ \\
\hline $\mathrm{C} 21$ & $84(5)$ & $52(4)$ & $51(4)$ & $-19(3)$ & $4(4)$ & $-9(4)$ \\
\hline $\mathrm{C} 22$ & 101(6) & $84(6)$ & $49(5)$ & $-13(4)$ & $-23(4)$ & $-6(5)$ \\
\hline $\mathrm{C} 23$ & $103(6)$ & $69(5)$ & $58(5)$ & $-2(4)$ & $-32(4)$ & $7(4)$ \\
\hline $\mathrm{C} 24$ & $78(5)$ & $57(4)$ & $52(4)$ & $2(3)$ & $-25(4)$ & $6(4)$ \\
\hline $\mathrm{C} 25$ & $43(3)$ & $35(3)$ & $33(3)$ & $2(2)$ & $-2(2)$ & $-1(2)$ \\
\hline C26 & $36.3(11)$ & $47.9(10)$ & $79.5(14)$ & $-7.0(9)$ & $2.1(9)$ & $-5.9(8)$ \\
\hline C26' & $36.3(11)$ & $47.9(10)$ & $79.5(14)$ & $-7.0(9)$ & $2.1(9)$ & $-5.9(8)$ \\
\hline $\mathrm{C} 27$ & $61(4)$ & $41(4)$ & $69(5)$ & $2(3)$ & $18(3)$ & $14(3)$ \\
\hline $\mathrm{C} 28$ & $75(5)$ & $38(4)$ & $68(5)$ & $-9(3)$ & $10(4)$ & $-3(3)$ \\
\hline $\mathrm{C} 29$ & $39(4)$ & $56(4)$ & $41(4)$ & $2(3)$ & 1(3) & $-6(3)$ \\
\hline C30 & $54(4)$ & $95(5)$ & $44(4)$ & $-8(4)$ & $-3(3)$ & $-8(4)$ \\
\hline
\end{tabular}

Table S8 Bond Lengths for 3am.

\begin{tabular}{|c|c|c|c|c|c|}
\hline \multicolumn{2}{|c|}{ Atom Atom } & \multirow{2}{*}{$\begin{array}{r}\text { Length/Å } \\
1.413(6)\end{array}$} & \multicolumn{2}{|c|}{ Atom Atom } & \multirow{2}{*}{$\begin{array}{r}\text { Length/ } \mathbf{\AA} \\
1.373(7)\end{array}$} \\
\hline $\mathrm{N} 1$ & $\mathrm{C} 2$ & & $\mathrm{C} 5$ & C6 & \\
\hline N1 & $\mathrm{C} 14$ & $1.350(7)$ & C6 & $\mathrm{C} 7$ & $1.368(8)$ \\
\hline $\mathrm{N} 2$ & $\mathrm{C} 17$ & $1.407(6)$ & $\mathrm{C} 7$ & $\mathrm{C} 8$ & $1.359(8)$ \\
\hline
\end{tabular}




\begin{tabular}{|c|c|c|c|c|c|}
\hline $\mathrm{N} 2$ & $\mathrm{C} 29$ & $1.344(7)$ & $\mathrm{C} 8$ & C9 & $1.373(7)$ \\
\hline $\mathrm{O} 1$ & $\mathrm{C} 1$ & $1.369(6)$ & $\mathrm{C} 10$ & $\mathrm{C} 11$ & $1.392(7)$ \\
\hline $\mathrm{O} 1$ & $\mathrm{C} 5$ & $1.382(6)$ & $\mathrm{C} 10$ & C11' & $1.394(10)$ \\
\hline $\mathrm{O} 2$ & $\mathrm{C} 1$ & $1.202(6)$ & $\mathrm{C} 11$ & $\mathrm{C} 12$ & $1.379(7)$ \\
\hline $\mathrm{O} 3$ & $\mathrm{C} 14$ & $1.217(6)$ & $\mathrm{C} 11^{\prime}$ & C13 & $1.393(10)$ \\
\hline $\mathrm{O} 4$ & $\mathrm{C} 16$ & $1.364(6)$ & $\mathrm{C} 12$ & C13 & $1.348(7)$ \\
\hline $\mathrm{O} 4$ & $\mathrm{C} 20$ & $1.372(6)$ & $\mathrm{C} 14$ & $\mathrm{C} 15$ & $1.494(7)$ \\
\hline O5 & $\mathrm{C} 16$ & $1.198(6)$ & $\mathrm{C} 16$ & C17 & $1.455(8)$ \\
\hline O6 & $\mathrm{C} 29$ & $1.224(6)$ & $\mathrm{C} 17$ & $\mathrm{C} 18$ & $1.349(7)$ \\
\hline S1 & $\mathrm{C} 10$ & $1.703(5)$ & C18 & C19 & $1.463(7)$ \\
\hline S1 & $\mathrm{C} 13$ & $1.662(5)$ & $\mathrm{C} 18$ & $\mathrm{C} 25$ & $1.468(7)$ \\
\hline S2 & $\mathrm{C} 25$ & $1.693(5)$ & C19 & $\mathrm{C} 20$ & $1.374(7)$ \\
\hline S2 & $\mathrm{C} 28$ & $1.669(6)$ & C19 & $\mathrm{C} 24$ & $1.397(7)$ \\
\hline S1' & $\mathrm{C} 10$ & $1.658(9)$ & $\mathrm{C} 20$ & $\mathrm{C} 21$ & $1.379(7)$ \\
\hline S1' & $\mathrm{C} 12$ & $1.638(9)$ & $\mathrm{C} 21$ & $\mathrm{C} 22$ & $1.367(9)$ \\
\hline$S 2^{\prime}$ & $\mathrm{C} 25$ & $1.630(8)$ & $\mathrm{C} 22$ & $\mathrm{C} 23$ & $1.354(9)$ \\
\hline$S 2^{\prime}$ & $\mathrm{C} 27$ & $1.614(8)$ & $\mathrm{C} 23$ & $\mathrm{C} 24$ & $1.377(8)$ \\
\hline $\mathrm{C} 1$ & $\mathrm{C} 2$ & $1.451(8)$ & $\mathrm{C} 25$ & $\mathrm{C} 26$ & $1.399(7)$ \\
\hline $\mathrm{C} 2$ & $\mathrm{C} 3$ & $1.357(7)$ & $\mathrm{C} 25$ & $\mathrm{C} 26^{\prime}$ & $1.388(10)$ \\
\hline $\mathrm{C} 3$ & $\mathrm{C} 4$ & $1.461(7)$ & $\mathrm{C} 26$ & $\mathrm{C} 27$ & $1.384(7)$ \\
\hline $\mathrm{C} 3$ & $\mathrm{C} 10$ & $1.468(7)$ & $\mathrm{C} 26^{\prime}$ & $\mathrm{C} 28$ & $1.397(10)$ \\
\hline $\mathrm{C} 4$ & C5 & $1.375(7)$ & $\mathrm{C} 27$ & $\mathrm{C} 28$ & $1.344(7)$ \\
\hline $\mathrm{C} 4$ & C9 & $1.404(7)$ & $\mathrm{C} 29$ & $\mathrm{C} 30$ & $1.492(7)$ \\
\hline
\end{tabular}

Table S9 Bond Angles for 3am.

\begin{tabular}{lllrllllr}
\multicolumn{2}{c}{ Atom Atom Atom } & \multicolumn{1}{c}{ Angle $^{\circ}$} & \multicolumn{2}{c}{ Atom Atom Atom } & Angle $^{\circ}$ \\
$\mathrm{C} 14$ & $\mathrm{~N} 1$ & $\mathrm{C} 2$ & $121.1(5)$ & $\mathrm{C} 12$ & $\mathrm{C} 13$ & $\mathrm{C} 11^{\prime}$ & $98.0(9)$ \\
$\mathrm{C} 29$ & $\mathrm{~N} 2$ & $\mathrm{C} 17$ & $122.0(5)$ & $\mathrm{N} 1$ & $\mathrm{C} 14$ & $\mathrm{C} 15$ & $116.4(5)$ \\
$\mathrm{C} 1$ & $\mathrm{O} 1$ & $\mathrm{C} 5$ & $121.5(4)$ & $\mathrm{O} 3$ & $\mathrm{C} 14$ & $\mathrm{~N} 1$ & $122.2(5)$ \\
$\mathrm{C} 16$ & $\mathrm{O} 4$ & $\mathrm{C} 20$ & $121.6(4)$ & $\mathrm{O} 3$ & $\mathrm{C} 14$ & $\mathrm{C} 15$ & $121.3(6)$ \\
$\mathrm{C} 13$ & $\mathrm{~S} 1$ & $\mathrm{C} 10$ & $93.3(3)$ & $\mathrm{O} 4$ & $\mathrm{C} 16$ & $\mathrm{C} 17$ & $117.0(5)$ \\
$\mathrm{C} 28$ & $\mathrm{~S} 2$ & $\mathrm{C} 25$ & $93.2(3)$ & $\mathrm{O} 5$ & $\mathrm{C} 16$ & $\mathrm{O} 4$ & $118.1(5)$ \\
$\mathrm{C} 12$ & $\mathrm{~S} 1$ & $\mathrm{C} 10$ & $90.8(5)$ & $\mathrm{O} 5$ & $\mathrm{C} 16$ & $\mathrm{C} 17$ & $124.9(5)$ \\
$\mathrm{C} 27$ & $\mathrm{~S} 2$ & $\mathrm{C} 25$ & $92.9(5)$ & $\mathrm{N} 2$ & $\mathrm{C} 17$ & $\mathrm{C} 16$ & $113.5(4)$ \\
$\mathrm{O} 1$ & $\mathrm{C} 1$ & $\mathrm{C} 2$ & $117.2(5)$ & $\mathrm{C} 18$ & $\mathrm{C} 17$ & $\mathrm{~N} 2$ & $123.6(5)$ \\
$\mathrm{O} 2$ & $\mathrm{C} 1$ & $\mathrm{O} 1$ & $117.3(5)$ & $\mathrm{C} 18$ & $\mathrm{C} 17$ & $\mathrm{C} 16$ & $122.9(5)$ \\
$\mathrm{O} 2$ & $\mathrm{C} 1$ & $\mathrm{C} 2$ & $125.5(5)$ & $\mathrm{C} 17$ & $\mathrm{C} 18$ & $\mathrm{C} 19$ & $117.1(5)$ \\
$\mathrm{N} 1$ & $\mathrm{C} 2$ & $\mathrm{C} 1$ & $113.5(4)$ & $\mathrm{C} 17$ & $\mathrm{C} 18$ & $\mathrm{C} 25$ & $122.7(4)$ \\
$\mathrm{C} 3$ & $\mathrm{C} 2$ & $\mathrm{~N} 1$ & $124.2(5)$ & $\mathrm{C} 19$ & $\mathrm{C} 18$ & $\mathrm{C} 25$ & $120.1(4)$
\end{tabular}




\begin{tabular}{|c|c|c|c|c|c|c|c|}
\hline $\mathrm{C} 3$ & $\mathrm{C} 2$ & $\mathrm{C} 1$ & $122.2(5)$ & $\mathrm{C} 20$ & $\mathrm{C} 19$ & $\mathrm{C} 18$ & 119.1(5) \\
\hline $\mathrm{C} 2$ & $\mathrm{C} 3$ & $\mathrm{C} 4$ & $117.3(5)$ & $\mathrm{C} 20$ & $\mathrm{C} 19$ & $\mathrm{C} 24$ & $116.9(5)$ \\
\hline $\mathrm{C} 2$ & $\mathrm{C} 3$ & $\mathrm{C} 10$ & $123.2(4)$ & $\mathrm{C} 24$ & $\mathrm{C} 19$ & $\mathrm{C} 18$ & $123.9(5)$ \\
\hline $\mathrm{C} 4$ & $\mathrm{C} 3$ & $\mathrm{C} 10$ & $119.4(4)$ & $\mathrm{O} 4$ & $\mathrm{C} 20$ & C19 & $121.9(5)$ \\
\hline $\mathrm{C} 5$ & $\mathrm{C} 4$ & $\mathrm{C} 3$ & $118.9(5)$ & $\mathrm{O} 4$ & $\mathrm{C} 20$ & $\mathrm{C} 21$ & $115.5(5)$ \\
\hline $\mathrm{C} 5$ & $\mathrm{C} 4$ & C9 & $116.9(5)$ & C19 & $\mathrm{C} 20$ & $\mathrm{C} 21$ & $122.5(5)$ \\
\hline C9 & $\mathrm{C} 4$ & $\mathrm{C} 3$ & $124.1(5)$ & $\mathrm{C} 22$ & $\mathrm{C} 21$ & $\mathrm{C} 20$ & 119.1(6) \\
\hline $\mathrm{C} 4$ & $\mathrm{C} 5$ & $\mathrm{O} 1$ & $121.4(5)$ & $\mathrm{C} 23$ & $\mathrm{C} 22$ & $\mathrm{C} 21$ & $119.9(6)$ \\
\hline C6 & $\mathrm{C} 5$ & $\mathrm{O} 1$ & $116.1(5)$ & $\mathrm{C} 22$ & $\mathrm{C} 23$ & $\mathrm{C} 24$ & $121.3(6)$ \\
\hline C6 & $\mathrm{C} 5$ & $\mathrm{C} 4$ & $122.4(5)$ & $\mathrm{C} 23$ & $\mathrm{C} 24$ & C19 & $120.2(6)$ \\
\hline $\mathrm{C} 7$ & C6 & $\mathrm{C} 5$ & $119.3(6)$ & $\mathrm{S} 2{ }^{\prime}$ & $\mathrm{C} 25$ & S2 & $118.7(4)$ \\
\hline $\mathrm{C} 8$ & $\mathrm{C} 7$ & C6 & $119.9(5)$ & $\mathrm{C} 18$ & $\mathrm{C} 25$ & S2 & $120.4(4)$ \\
\hline $\mathrm{C} 7$ & $\mathrm{C} 8$ & C9 & $121.0(6)$ & $\mathrm{C} 18$ & $\mathrm{C} 25$ & $\mathrm{~S} 2^{\prime}$ & $120.9(4)$ \\
\hline $\mathrm{C} 8$ & C9 & $\mathrm{C} 4$ & $120.3(5)$ & $\mathrm{C} 26$ & $\mathrm{C} 25$ & S2 & $107.6(4)$ \\
\hline S1' & $\mathrm{C} 10$ & S1 & $119.4(4)$ & $\mathrm{C} 26$ & $\mathrm{C} 25$ & $\mathrm{~S} 22^{\prime}$ & $11.2(5)$ \\
\hline $\mathrm{C} 3$ & $\mathrm{C} 10$ & S1 & $126.2(4)$ & $\mathrm{C} 26$ & $\mathrm{C} 25$ & $\mathrm{C} 18$ & $132.0(5)$ \\
\hline $\mathrm{C} 3$ & $\mathrm{C} 10$ & S1' & $113.7(5)$ & $\mathrm{C} 26^{\prime}$ & $\mathrm{C} 25$ & S2 & $20(2)$ \\
\hline $\mathrm{C} 11$ & $\mathrm{C} 10$ & S1 & $107.2(4)$ & $\mathrm{C} 26^{\prime}$ & $\mathrm{C} 25$ & $\mathrm{~S} 2^{\prime}$ & $102.6(12)$ \\
\hline $\mathrm{C} 11$ & $\mathrm{C} 10$ & $\mathrm{~S} 1^{\prime}$ & $12.6(5)$ & $\mathrm{C} 26^{\prime}$ & $\mathrm{C} 25$ & $\mathrm{C} 18$ & $134.8(8)$ \\
\hline $\mathrm{C} 11$ & $\mathrm{C} 10$ & $\mathrm{C} 3$ & $126.2(5)$ & $\mathrm{C} 26^{\prime}$ & $\mathrm{C} 25$ & $\mathrm{C} 26$ & $91.5(11)$ \\
\hline $\mathrm{C} 11$ & $\mathrm{C} 10$ & $\mathrm{C} 11^{\prime}$ & $92.9(8)$ & $\mathrm{C} 27$ & $\mathrm{C} 26$ & $\mathrm{C} 25$ & $115.4(6)$ \\
\hline C11' & $\mathrm{C} 10$ & S1 & $15.0(13)$ & $\mathrm{C} 25$ & C26' & $\mathrm{C} 28$ & $122.7(12)$ \\
\hline $\mathrm{C} 11^{\prime}$ & $\mathrm{C} 10$ & $\mathrm{~S} 1^{\prime}$ & $105.3(8)$ & $\mathrm{C} 26$ & $\mathrm{C} 27$ & $\mathrm{~S} 2^{\prime}$ & $11.3(5)$ \\
\hline $\mathrm{C} 11^{\prime}$ & $\mathrm{C} 10$ & $\mathrm{C} 3$ & $140.8(8)$ & $\mathrm{C} 28$ & $\mathrm{C} 27$ & $\mathrm{~S} 22^{\prime}$ & $121.3(5)$ \\
\hline $\mathrm{C} 12$ & $\mathrm{C} 11$ & $\mathrm{C} 10$ & $115.7(6)$ & $\mathrm{C} 28$ & $\mathrm{C} 27$ & $\mathrm{C} 26$ & $110.1(6)$ \\
\hline $\mathrm{C} 13$ & C11' & $\mathrm{C} 10$ & $122.8(12)$ & C26' & $\mathrm{C} 28$ & S2 & $21(2)$ \\
\hline $\mathrm{C} 11$ & $\mathrm{C} 12$ & $\mathrm{~S} 1^{\prime}$ & $13.0(5)$ & $\mathrm{C} 27$ & $\mathrm{C} 28$ & S2 & $113.7(4)$ \\
\hline $\mathrm{C} 13$ & $\mathrm{C} 12$ & $\mathrm{~S} 1^{\prime}$ & $122.6(5)$ & $\mathrm{C} 27$ & $\mathrm{C} 28$ & C26' & $96.8(11)$ \\
\hline $\mathrm{C} 13$ & $\mathrm{C} 12$ & $\mathrm{C} 11$ & $110.1(6)$ & $\mathrm{N} 2$ & $\mathrm{C} 29$ & $\mathrm{C} 30$ & $116.3(5)$ \\
\hline $\mathrm{C} 11^{\prime}$ & $\mathrm{C} 13$ & S1 & $16.2(12)$ & O6 & $\mathrm{C} 29$ & $\mathrm{~N} 2$ & $122.2(5)$ \\
\hline $\mathrm{C} 12$ & C13 & S1 & $113.6(4)$ & O6 & $\mathrm{C} 29$ & $\mathrm{C} 30$ & $121.5(6)$ \\
\hline
\end{tabular}

Table S10 Torsion Angles for 3am.

\begin{tabular}{|c|c|c|c|c|c|}
\hline $\mathbf{A}$ & B & D & Angle $/^{\circ}$ & A $\mathbf{B}$ & Angle $/^{\circ}$ \\
\hline N1 & $\mathrm{C} 2$ & $\mathrm{C} 3 \mathrm{C} 4$ & $-171.0(5)$ & C11 C12 C13 S1 & $1.2(8)$ \\
\hline N1 & $\mathrm{C} 2$ & C3 C10 & $11.4(8)$ & C11 C12 C13 C11' & $-3(3)$ \\
\hline $\mathrm{N} 2$ & $\mathrm{C} 17$ & C18 C19 & $-172.8(5)$ & C11'C10 C11 C12 & $4(3)$ \\
\hline $\mathrm{N} 2$ & $\mathrm{C} 17$ & $\mathrm{C} 18 \mathrm{C} 25$ & $10.1(8)$ & C12 S1' C10 S1 & $-5.5(10)$ \\
\hline $\mathrm{O} 1$ & $\mathrm{C} 1$ & $\mathrm{C} 2 \mathrm{~N} 1$ & $177.4(5)$ & C12 S1' C10 C3 & $-176.7(5)$ \\
\hline
\end{tabular}




\begin{tabular}{|c|c|c|c|c|}
\hline $\mathrm{O} 1$ & $\mathrm{C} 1 \quad \mathrm{C} 2 \quad \mathrm{C} 3$ & $-6.4(8)$ & C12 S1' C10 C11 & 11(3) \\
\hline $\mathrm{O} 1$ & C5 $\quad$ C6 $\quad$ C7 & $177.9(5)$ & C12 S1' C10 C11' & $0(3)$ \\
\hline $\mathrm{O} 2$ & $\begin{array}{lll}\mathrm{C} 1 & \mathrm{C} 2 & \mathrm{~N} 1\end{array}$ & $-2.7(9)$ & C13 S1 C10 S1' & $4.7(8)$ \\
\hline $\mathrm{O} 2$ & $\mathrm{C} 1 \quad \mathrm{C} 2 \quad \mathrm{C} 3$ & $173.5(6)$ & $\mathrm{C} 13 \mathrm{~S} 1 \quad \mathrm{C} 10 \mathrm{C} 3$ & $174.6(5)$ \\
\hline $\mathrm{O} 4$ & C16 C17 N2 & $172.2(5)$ & C13 S1 C10 C11 & $0.9(5)$ \\
\hline $\mathrm{O} 4$ & C16 C17 C18 & $-7.8(8)$ & C13 S1 C10 C11' & $-17(10)$ \\
\hline $\mathrm{O} 4$ & $\mathrm{C} 20 \mathrm{C} 21 \mathrm{C} 22$ & $-177.0(7)$ & $\mathrm{C} 14 \mathrm{~N} 1 \mathrm{C} 2 \mathrm{C} 1$ & $-106.2(6)$ \\
\hline O5 & C16 C17 N2 & $-7.3(9)$ & $\mathrm{C} 14 \mathrm{~N} 1 \mathrm{C} 2 \mathrm{C} 3$ & 77.7(7) \\
\hline O5 & $\mathrm{C} 16 \mathrm{C} 17 \mathrm{C} 18$ & $172.7(6)$ & C16 O4 C20 C19 & $1.4(9)$ \\
\hline S1 & $\mathrm{C} 10 \mathrm{C} 11 \mathrm{C} 12$ & $-0.4(9)$ & $\mathrm{C} 16 \mathrm{O} 4 \mathrm{C} 20 \mathrm{C} 21$ & $178.7(6)$ \\
\hline S1 & $\mathrm{C} 10 \mathrm{C} 11^{\prime} \mathrm{C} 13$ & $156(15)$ & C16 C17 C18 C19 & $7.1(8)$ \\
\hline S2 & C25 C26 C27 & $0.3(10)$ & C16 C17 C18 C25 & $-170.0(5)$ \\
\hline S2 & C25 C26'C28 & $-125(9)$ & C17 N2 C29 O6 & $-4.4(9)$ \\
\hline S1' & $\mathrm{C} 10 \mathrm{C} 11 \mathrm{C} 12$ & $-165(5)$ & C17 N2 C29 C30 & $175.2(5)$ \\
\hline S1' & $\mathrm{C} 10 \mathrm{C} 11^{\prime} \mathrm{C} 13$ & $-5(6)$ & C17 C18 C19 C20 & $-2.2(8)$ \\
\hline S1' & C12 C13 S1 & $-2.5(10)$ & C17 C18 C19 C24 & $-180.0(6)$ \\
\hline S1' & C12 C13 C11' & $-7(3)$ & C17 C18 C25 S2 & $51.3(7)$ \\
\hline$S 2^{\prime}$ & $\mathrm{C} 25 \mathrm{C} 26 \mathrm{C} 27$ & $177(6)$ & C17 C18 C25 S2' & $-129.4(9)$ \\
\hline S2' & $\mathrm{C} 25 \mathrm{C} 26^{\prime} \mathrm{C} 28$ & $22(5)$ & C17 C18 C25 C26 & $-130.6(8)$ \\
\hline S2' & $\mathrm{C} 27 \mathrm{C} 28 \mathrm{~S} 2$ & $-2.5(11)$ & C17 C18 C25 C26' & $69(4)$ \\
\hline S2' & C27 C28 C26' & $10(2)$ & C18 C19 C20 O4 & $-2.1(9)$ \\
\hline $\mathrm{C} 1$ & $\mathrm{O} 1 \quad \mathrm{C} 5 \quad \mathrm{C} 4$ & $6.7(8)$ & C18 C19 C20 C21 & $-179.2(6)$ \\
\hline $\mathrm{C} 1$ & $\mathrm{O} 1 \mathrm{C} 5 \mathrm{C} 6$ & $-172.7(6)$ & C18 C19 C24 C23 & $180.0(7)$ \\
\hline $\mathrm{C} 1$ & $\mathrm{C} 2 \quad \mathrm{C} 3 \quad \mathrm{C} 4$ & $13.2(8)$ & $\mathrm{C} 18 \mathrm{C} 25 \mathrm{C} 26 \mathrm{C} 27$ & $-177.9(6)$ \\
\hline $\mathrm{C} 1$ & $\mathrm{C} 2 \quad \mathrm{C} 3 \quad \mathrm{C} 10$ & $-164.3(5)$ & C18 C25 C26' C28 & $-174(2)$ \\
\hline $\mathrm{C} 2$ & $\mathrm{~N} 1 \mathrm{C} 14 \mathrm{O} 3$ & $-8.1(8)$ & C19 C18 C25 S2 & $-125.7(5)$ \\
\hline $\mathrm{C} 2$ & N1 C14 C15 & $173.0(5)$ & C19 C18 C25 S2' & $53.6(10)$ \\
\hline $\mathrm{C} 2$ & C3 C4 C5 & $-10.3(8)$ & C19 C18 C25 C26 & $52.3(10)$ \\
\hline $\mathrm{C} 2$ & C3 C4 C9 & $166.6(5)$ & C19 C18 C25 C26' & $-108(4)$ \\
\hline $\mathrm{C} 2$ & C3 C10 S1 & $-38.4(7)$ & C19 C20 C21 C22 & $0.2(11)$ \\
\hline $\mathrm{C} 2$ & C3 C10 S1' & $132.0(8)$ & $\mathrm{C} 20 \mathrm{O} 4 \mathrm{C} 16 \mathrm{O} 5$ & $-177.2(5)$ \\
\hline $\mathrm{C} 2$ & C3 C10 C11 & $134.1(7)$ & $\mathrm{C} 20 \mathrm{O} 4 \mathrm{C} 16 \mathrm{C} 17$ & $3.2(8)$ \\
\hline $\mathrm{C} 2$ & C3 C10 C11' & $-43(5)$ & C20 C19 C24 C23 & $2.1(10)$ \\
\hline $\mathrm{C} 3$ & $\mathrm{C} 4 \quad \mathrm{C} 5 \mathrm{O} 1$ & $0.6(8)$ & $\mathrm{C} 20 \mathrm{C} 21 \mathrm{C} 22 \mathrm{C} 23$ & $-0.2(12)$ \\
\hline $\mathrm{C} 3$ & $\mathrm{C} 4 \mathrm{C} 5 \mathrm{C} 6$ & $179.9(6)$ & C21 C22 C23 C24 & $1.1(14)$ \\
\hline $\mathrm{C} 3$ & $\begin{array}{lll}\mathrm{C} 4 & \mathrm{C} 9 & \mathrm{C} 8\end{array}$ & $-178.7(5)$ & C22 C23 C24 C19 & $-2.2(13)$ \\
\hline $\mathrm{C} 3$ & C10 C11 C12 & $-174.1(6)$ & C24 C19 C20 O4 & $175.8(6)$ \\
\hline $\mathrm{C} 3$ & $\mathrm{C} 10 \mathrm{C} 11^{\prime} \mathrm{C} 13$ & $170.5(19)$ & C24 C19 C20 C21 & $-1.2(9)$ \\
\hline $\mathrm{C} 4$ & C3 C10 S1 & 144.1(4) & $\mathrm{C} 25 \mathrm{~S} 2 \mathrm{C}^{28} \mathrm{C} 26^{\prime}$ & $-34(4)$ \\
\hline $\mathrm{C} 4$ & C3 C10 S1' & $-45.5(9)$ & $\mathrm{C} 25 \mathrm{~S} 2 \quad \mathrm{C} 28 \mathrm{C} 27$ & $2.8(6)$ \\
\hline
\end{tabular}




\begin{tabular}{|c|c|c|c|c|}
\hline $\mathrm{C} 4 \mathrm{C} 3$ & $\mathrm{C} 10 \mathrm{C} 11$ & $-43.3(8)$ & $\mathrm{C} 25 \mathrm{~S} 22^{\prime} \mathrm{C} 27 \mathrm{C} 26$ & $3(5)$ \\
\hline $\mathrm{C} 4 \mathrm{C} 3$ & $\mathrm{C} 10 \mathrm{C} 11^{\prime}$ & $140(4)$ & $\mathrm{C} 25 \mathrm{~S} 2^{\prime} \mathrm{C} 27 \mathrm{C} 28$ & $0.7(13)$ \\
\hline $\mathrm{C} 4 \mathrm{C} 5$ & C6 C7 & $-1.5(10)$ & C25 C18 C19 C20 & $175.0(5)$ \\
\hline C5 O1 & $\mathrm{C} 1 \mathrm{O} 2$ & $176.2(5)$ & C25 C18 C19 C24 & $-2.8(9)$ \\
\hline $\mathrm{C} 5 \mathrm{O} 1$ & $\mathrm{C} 1 \mathrm{C} 2$ & $-4.0(8)$ & C25 C26 C27 S2' & $-177(6)$ \\
\hline $\mathrm{C} 5 \mathrm{C} 4$ & C9 $\mathrm{C} 8$ & $-1.7(8)$ & $\mathrm{C} 25 \mathrm{C} 26 \mathrm{C} 27 \mathrm{C} 28$ & $1.7(11)$ \\
\hline C5 C6 & $\mathrm{C} 7 \quad \mathrm{C} 8$ & $-0.9(10)$ & C25 C26'C28 S2 & $127(9)$ \\
\hline C6 C7 & $\mathrm{C} 8 \quad \mathrm{C} 9$ & $1.9(10)$ & C25 C26'C28 C27 & $-20(5)$ \\
\hline $\mathrm{C} 7 \mathrm{C} 8$ & $\mathrm{C} 9 \mathrm{C} 4$ & $-0.5(9)$ & C26 C25 C26' C28 & $20(5)$ \\
\hline $\mathrm{C} 9 \mathrm{C} 4$ & C5 O1 & $-176.6(5)$ & $\mathrm{C} 26 \mathrm{C} 27 \mathrm{C} 28 \mathrm{~S} 2$ & $-2.9(9)$ \\
\hline $\mathrm{C} 9 \mathrm{C} 4$ & C5 C6 & $2.8(9)$ & C26 C27 C28 C26' & $9(2)$ \\
\hline $\mathrm{C} 10 \mathrm{~S} 1$ & C13 C11' & $15(10)$ & C26' C25 C26 C27 & $-11(2)$ \\
\hline $\mathrm{C} 10 \mathrm{~S} 1$ & $\mathrm{C} 13 \mathrm{C} 12$ & $-1.3(5)$ & C27 S2' C25 S2 & $1.4(11)$ \\
\hline C10S1' & $\mathrm{C} 12 \mathrm{C} 11$ & $-11(3)$ & C27 S2' C25 C18 & $-177.9(6)$ \\
\hline C10S1' & C12 C13 & $4.8(11)$ & C27 S2' C25 C26 & $-3(5)$ \\
\hline $\mathrm{C} 10 \mathrm{C} 3$ & $\mathrm{C} 4 \mathrm{C} 5$ & $167.4(5)$ & C27 S2' C25 C26' & $-11(3)$ \\
\hline $\mathrm{C} 10 \mathrm{C} 3$ & $\mathrm{C} 4 \quad \mathrm{C} 9$ & $-15.7(8)$ & $\mathrm{C} 28 \mathrm{~S} 2 \quad \mathrm{C} 25 \mathrm{~S} 2^{\prime}$ & $-2.5(9)$ \\
\hline $\mathrm{C} 10 \mathrm{C} 11$ & C12 S1' & $165(4)$ & C28 S2 C25 C18 & $176.8(5)$ \\
\hline $\mathrm{C} 10 \mathrm{C} 11$ & $\mathrm{C} 12 \mathrm{C} 13$ & $-0.5(10)$ & C28 S2 C25 C26 & $-1.7(6)$ \\
\hline $\mathrm{C} 10 \mathrm{C} 11$ & 'C13 S1 & $-158(14)$ & C28 S2 C25 C26' & $35(5)$ \\
\hline $\mathrm{C} 10 \mathrm{C} 11$ & 'C13 C12 & $7(6)$ & $\mathrm{C} 29 \mathrm{~N} 2 \mathrm{C} 17 \mathrm{C} 16$ & $-119.5(6)$ \\
\hline $\mathrm{C} 11 \mathrm{C} 10$ & $\mathrm{C} 11^{\prime} \mathrm{C} 13$ & $-7(5)$ & C29 N2 C17 C18 & $60.5(8)$ \\
\hline
\end{tabular}

Table S11 Hydrogen Atom Coordinates $\left(\AA \times 10^{4}\right)$ and Isotropic Displacement Parameters $\left(\AA^{2} \times 10^{3}\right)$ for $3 \mathrm{am}$.

\begin{tabular}{lrrrr} 
Atom & \multicolumn{2}{r}{$\boldsymbol{y}$} & $\boldsymbol{z}$ & $\mathbf{U}(\mathbf{e q})$ \\
H1 & 7837 & 8940 & 3443 & 46 \\
H2 & 2926 & 8852 & 3489 & 49 \\
H6 & 4498 & 7438 & -92 & 65 \\
H7 & 2608 & 8040 & -853 & 67 \\
H8 & 1869 & 9104 & -300 & 58 \\
H9 & 3058 & 9618 & 969 & 49 \\
H11 & 5083 & 10407 & 1098 & 55 \\
H11' & 4945 & 10070 & 3610 & 55 \\
H12 & 5023 & 11561 & 1828 & 63 \\
H13 & 5044 & 11430 & 3431 & 66 \\
H15A & 6796 & 9217 & 5370 & 82 \\
H15B & 8129 & 8967 & 4925 & 44 \\
H15C & 7195 & 8399 & 5362 &
\end{tabular}




$\begin{array}{lrrrr}\text { H21 } & -263 & 7296 & -9 & 75 \\ \text { H22 } & -1920 & 7928 & -946 & 96 \\ \text { H23 } & -2556 & 9056 & -555 & 96 \\ \text { H24 } & -1504 & 9600 & 737 & 78 \\ \text { H26 } & -1966 & 9967 & 2256 & 66 \\ \text { H26 } & 1826 & 10437 & 2102 & 66 \\ \text { H27 } & -1646 & 11217 & 2673 & 67 \\ \text { H28 } & 790 & 11545 & 2792 & 72 \\ \text { H30A } & 1627 & 9433 & 5316 & 98 \\ \text { H30B } & 3024 & 9227 & 4912 & 98 \\ \text { H30C } & 2145 & 8631 & 5343 & 98\end{array}$

Table S12 Atomic Occupancy for 3am.

$\begin{array}{lrlrlr}\text { Atom } & \text { Occupancy } & \text { Atom } & \text { Occupancy } & \text { Atom } & \text { Occupancy } \\ \text { S1 } & 0.869(4) & \text { S2 } & 0.859(4) & \text { S1' } & 0.131(4) \\ \text { S2' } & 0.141(4) & \text { C11 } & 0.869(4) & \text { H11 } & 0.869(4) \\ \text { C11' } & 0.131(4) & \text { H11' } & 0.131(4) & \text { C26 } & 0.859(4) \\ \text { H26 } & 0.859(4) & \text { C26' } & 0.141(4) & \text { H26' } & 0.141(4)\end{array}$

\section{Crystallographic data 3 ao}

Table S13 Crystal data and structure refinement for $3 \mathrm{ao.}$

$\begin{array}{ll}\text { Identification code } & \text { 3ao } \\ \text { Empirical formula } & \mathrm{C}_{17} \mathrm{H}_{23} \mathrm{NO}_{4} \\ \text { Formula weight } & 305.36 \\ \text { Temperature/K } & 298.15 \\ \text { Crystal system } & \text { tetragonal } \\ \text { Space group } & \mathrm{I} 4{ }_{1} / \mathrm{a} \\ \mathrm{a} / \AA & 18.5878(16) \\ \mathrm{b} / \AA & 18.5878(16) \\ \mathrm{c} / \AA & 20.3165(18) \\ \alpha /{ }^{\circ} & 90.00 \\ \beta /{ }^{\circ} & 90.00 \\ \gamma /{ }^{\circ} & 90.00 \\ \mathrm{Volume} / \AA^{3} & 7019.5(11) \\ \mathrm{Z} & 16 \\ \rho_{\text {calc }} / \mathrm{cm}^{3} & ? \\ \mu / \mathrm{mm}^{-1} & 0.082 \\ \mathrm{~F}(000) & 2624.0\end{array}$




$\begin{array}{ll}\text { Crystal size } / \mathrm{mm}^{3} & 0.4 \times 0.38 \times 0.35 \\ \text { Radiation } & \operatorname{MoK} \alpha(\lambda=0.71073) \\ 2 \Theta \text { range for data collection } /{ }^{\circ} 4.38 \text { to } 50.04 & -22 \leq \mathrm{h} \leq 19,-21 \leq \mathrm{k} \leq 22,-15 \leq 1 \leq 24 \\ \text { Index ranges } & 16867 \\ \text { Reflections collected } & 3099\left[\mathrm{R}_{\text {int }}=0.0631, \mathrm{R}_{\text {sigma }}=0.0454\right] \\ \text { Independent reflections } & 3099 / 48 / 203 \\ \text { Data/restraints/parameters } & 1.016 \\ \text { Goodness-of-fit on } \mathrm{F}^{2} & \mathrm{R}_{1}=0.0672, \mathrm{wR}_{2}=0.1920 \\ \text { Final R indexes [I }>=2 \sigma(\mathrm{I})] & \mathrm{R}_{1}=0.1269, \mathrm{wR}_{2}=0.2195 \\ \text { Final } \mathrm{R} \text { indexes [all data }] & \text { Largest diff. peak/hole } / \mathrm{e} \AA^{-3} 0.44 /-0.27\end{array}$

Table S14 Fractional Atomic Coordinates $\left(\times 10^{4}\right)$ and Equivalent Isotropic Displacement Parameters $\left(\AA^{2} \times 10^{3}\right)$ for 3 ao. $U_{\text {eq }}$ is defined as $1 / 3$ of of the trace of the orthogonalised $U_{\text {IJ }}$ tensor.

\begin{tabular}{lllll}
\multicolumn{2}{l}{ Atom $\boldsymbol{x}$} & $\boldsymbol{y}$ & $\boldsymbol{z}$ & $\mathbf{U}(\mathbf{e q})$ \\
$\mathrm{N} 1$ & $1449.5(16)$ & $5218.6(15)$ & $665.8(14)$ & $69.8(9)$ \\
$\mathrm{O} 1$ & $2333.8(15)$ & $6314.1(15)$ & $594.5(14)$ & $89.5(9)$ \\
$\mathrm{O} 2$ & $2831.7(18)$ & $5959.8(16)$ & $-343.3(16)$ & $104.1(11)$ \\
$\mathrm{O} 3$ & $2076.5(14)$ & $3919.3(13)$ & $1249.6(13)$ & $86.1(9)$ \\
$\mathrm{O} 4$ & $771.8(15)$ & $5747.4(16)$ & $-101.4(14)$ & $95.4(10)$ \\
$\mathrm{C} 1$ & $2455(2)$ & $5833(2)$ & $119(2)$ & $76.0(11)$ \\
$\mathrm{C} 2$ & $2054(2)$ & $5148(2)$ & $244.1(18)$ & $67.5(10)$ \\
$\mathrm{C} 3$ & $2228.3(19)$ & $4511(2)$ & $-16.6(19)$ & $68.9(10)$ \\
$\mathrm{C} 4$ & $1789.0(19)$ & $3864.5(19)$ & $124.0(19)$ & $66.4(10)$ \\
$\mathrm{C} 5$ & $1725.0(19)$ & $3575(2)$ & $751(2)$ & $67.9(10)$ \\
$\mathrm{C} 6$ & $1331(2)$ & $2952(2)$ & $857(2)$ & $75.6(11)$ \\
$\mathrm{C} 7$ & $985(2)$ & $2624(2)$ & $341(2)$ & $87.6(13)$ \\
$\mathrm{C} 8$ & $1032(2)$ & $2908(3)$ & $-279(2)$ & $93.4(13)$ \\
$\mathrm{C} 9$ & $1437(2)$ & $3519(2)$ & $-383(2)$ & $82.9(12)$ \\
$\mathrm{C} 10$ & $2869(2)$ & $4378(3)$ & $-440(3)$ & $100.6(15)$ \\
$\mathrm{C} 11$ & $3486(3)$ & $4016(4)$ & $-19(4)$ & $166(2)$ \\
$\mathrm{C} 12$ & $4130(4)$ & $3912(5)$ & $-300(5)$ & $201(3)$ \\
$\mathrm{C} 13$ & $4702(5)$ & $3662(6)$ & $185(5)$ & $233(3)$ \\
$\mathrm{C} 14$ & $5336(7)$ & $3666(8)$ & $-168(7)$ & $309(5)$ \\
$\mathrm{C} 15$ & $848(2)$ & $5540(2)$ & $468(2)$ & $69.9(10)$ \\
$\mathrm{C} 16$ & $271(2)$ & $5649(2)$ & $970(2)$ & $88.7(13)$ \\
$\mathrm{C} 17$ & $2673(3)$ & $7001(2)$ & $507(2)$ & $106.6(16)$
\end{tabular}


Table S15 Anisotropic Displacement Parameters $\left(\AA^{2} \times 10^{3}\right)$ for 3ao. The Anisotropic displacement factor exponent takes the form: $-2 \pi^{2}\left[h^{2} a * 2 U_{11}+2 h k a * b * U_{12}+\ldots\right]$.

\begin{tabular}{lllllll}
\multicolumn{2}{c}{ Atom $\mathbf{U}_{\mathbf{1 1}}$} & $\mathbf{U}_{\mathbf{2 2}}$ & $\mathbf{U}_{\mathbf{3 3}}$ & $\mathbf{U}_{\mathbf{2 3}}$ & $\mathbf{U}_{\mathbf{1 3}}$ & $\mathbf{U}_{\mathbf{1 2}}$ \\
$\mathrm{N} 1$ & $79(2)$ & $75.2(19)$ & $55.3(18)$ & $12.3(15)$ & $7.4(17)$ & $12.3(16)$ \\
$\mathrm{O} 1$ & $112(2)$ & $75.9(18)$ & $81(2)$ & $1.7(16)$ & $6.4(16)$ & $-4.1(16)$ \\
$\mathrm{O} 2$ & $119(2)$ & $108(2)$ & $85(2)$ & $1.8(18)$ & $27(2)$ & $-28.3(18)$ \\
$\mathrm{O} 3$ & $115(2)$ & $81.0(17)$ & $62.1(17)$ & $3.0(14)$ & $-9.1(16)$ & $-10.7(15)$ \\
$\mathrm{O} 4$ & $94(2)$ & $126(2)$ & $66.0(18)$ & $22.2(18)$ & $7.7(15)$ & $26.3(17)$ \\
$\mathrm{C} 1$ & $78(3)$ & $83(3)$ & $67(3)$ & $9(2)$ & $-5(2)$ & $2(2)$ \\
$\mathrm{C} 2$ & $71(2)$ & $74(3)$ & $57(2)$ & $11.8(19)$ & $5.1(19)$ & $7.5(19)$ \\
$\mathrm{C} 3$ & $65(2)$ & $78(3)$ & $63(2)$ & $10(2)$ & $0.3(19)$ & $10(2)$ \\
$\mathrm{C} 4$ & $68(2)$ & $69(2)$ & $61(2)$ & $3(2)$ & $0.9(19)$ & $10.8(19)$ \\
$\mathrm{C} 5$ & $72(2)$ & $68(2)$ & $63(2)$ & $-3(2)$ & $-3(2)$ & $4.7(19)$ \\
$\mathrm{C} 6$ & $80(2)$ & $78(3)$ & $69(3)$ & $2(2)$ & $3(2)$ & $1(2)$ \\
$\mathrm{C} 7$ & $82(3)$ & $89(3)$ & $92(3)$ & $2(3)$ & $1(3)$ & $-8(2)$ \\
$\mathrm{C} 8$ & $94(3)$ & $102(3)$ & $84(3)$ & $-10(3)$ & $-13(3)$ & $-6(3)$ \\
$\mathrm{C} 9$ & $85(3)$ & $99(3)$ & $65(3)$ & $2(2)$ & $-5(2)$ & $3(2)$ \\
$\mathrm{C} 10$ & $82(3)$ & $99(3)$ & $122(4)$ & $-6(3)$ & $25(3)$ & $9(2)$ \\
$\mathrm{C} 11$ & $105(4)$ & $174(5)$ & $221(6)$ & $39(4)$ & $49(4)$ & $46(3)$ \\
$\mathrm{C} 12$ & $127(4)$ & $239(6)$ & $237(6)$ & $41(5)$ & $9(5)$ & $36(4)$ \\
$\mathrm{C} 13$ & $143(5)$ & $301(7)$ & $254(7)$ & $44(7)$ & $14(6)$ & $49(6)$ \\
$\mathrm{C} 14$ & $223(9)$ & $377(11)$ & $329(11)$ & $6(10)$ & $26(9)$ & $17(9)$ \\
$\mathrm{C} 15$ & $77(3)$ & $72(2)$ & $60(3)$ & $6(2)$ & $3(2)$ & $5(2)$ \\
$\mathrm{C} 16$ & $85(3)$ & $111(3)$ & $71(3)$ & $5(2)$ & $14(2)$ & $14(2)$ \\
$\mathrm{C} 17$ & $134(4)$ & $79(3)$ & $107(4)$ & $-4(3)$ & $-3(3)$ & $-17(3)$
\end{tabular}

Table S16 Bond Lengths for 3ao.

\begin{tabular}{lll}
\multicolumn{3}{c}{ Atom Atom Length/ } \\
$\mathrm{N} 1$ & $\mathrm{C} 2$ & $1.419(4)$ \\
$\mathrm{N} 1$ & $\mathrm{C} 15$ & $1.329(4)$ \\
$\mathrm{O} 1$ & $\mathrm{C} 1$ & $1.335(5)$ \\
$\mathrm{O} 1$ & $\mathrm{C} 17$ & $1.435(5)$ \\
$\mathrm{O} 2$ & $\mathrm{C} 1$ & $1.195(4)$ \\
$\mathrm{O} 3$ & $\mathrm{C} 5$ & $1.366(4)$ \\
$\mathrm{O} 4$ & $\mathrm{C} 15$ & $1.228(4)$ \\
$\mathrm{C} 1$ & $\mathrm{C} 2$ & $1.498(5)$ \\
$\mathrm{C} 2$ & $\mathrm{C} 3$ & $1.336(5)$ \\
$\mathrm{C} 3$ & $\mathrm{C} 4$ & $1.481(5)$ \\
$\mathrm{C} 3$ & $\mathrm{C} 10$ & $1.489(5)$
\end{tabular}

\begin{tabular}{lll}
\multicolumn{3}{c}{ Atom Atom Length/Å } \\
$\mathrm{C} 4$ & $\mathrm{C} 5$ & $1.387(5)$ \\
$\mathrm{C} 4$ & $\mathrm{C} 9$ & $1.379(5)$ \\
$\mathrm{C} 5$ & $\mathrm{C} 6$ & $1.386(5)$ \\
$\mathrm{C} 6$ & $\mathrm{C} 7$ & $1.374(5)$ \\
$\mathrm{C} 7$ & $\mathrm{C} 8$ & $1.367(6)$ \\
$\mathrm{C} 8$ & $\mathrm{C} 9$ & $1.380(6)$ \\
$\mathrm{C} 10$ & $\mathrm{C} 11$ & $1.581(8)$ \\
$\mathrm{C} 11$ & $\mathrm{C} 12$ & $1.341(8)$ \\
$\mathrm{C} 12$ & $\mathrm{C} 13$ & $1.522(11)$ \\
$\mathrm{C} 13$ & $\mathrm{C} 14$ & $1.379(13)$ \\
$\mathrm{C} 15$ & $\mathrm{C} 16$ & $1.494(5)$
\end{tabular}


Table S17 Bond Angles for 3ao.

Atom Atom Atom Angle/
$\begin{array}{llll} & \\ \mathrm{C} 15 & \mathrm{~N} 1 & \mathrm{C} 2 & 121.7(3) \\ \mathrm{C} 1 & \mathrm{O} 1 & \mathrm{C} 17 & 115.6(3) \\ \mathrm{O} 1 & \mathrm{C} 1 & \mathrm{C} 2 & 111.3(4) \\ \mathrm{O} 2 & \mathrm{C} 1 & \mathrm{O} 1 & 122.4(4) \\ \mathrm{O} 2 & \mathrm{C} 1 & \mathrm{C} 2 & 126.3(4) \\ \mathrm{N} 1 & \mathrm{C} 2 & \mathrm{C} 1 & 114.7(3) \\ \mathrm{C} 3 & \mathrm{C} 2 & \mathrm{~N} 1 & 120.9(3) \\ \mathrm{C} 3 & \mathrm{C} 2 & \mathrm{C} 1 & 124.4(4) \\ \mathrm{C} 2 & \mathrm{C} 3 & \mathrm{C} 4 & 120.6(3) \\ \mathrm{C} 2 & \mathrm{C} 3 & \mathrm{C} 10 & 124.7(4) \\ \mathrm{C} 4 & \mathrm{C} 3 & \mathrm{C} 10 & 114.7(3) \\ \mathrm{C} 5 & \mathrm{C} 4 & \mathrm{C} 3 & 122.6(4) \\ \mathrm{C} 9 & \mathrm{C} 4 & \mathrm{C} 3 & 119.7(4) \\ \mathrm{C} 9 & \mathrm{C} 4 & \mathrm{C} 5 & 117.7(4)\end{array}$

Atom Atom Atom Angle $/^{\circ}$
$\begin{array}{llll}\text { O3 } & \mathrm{C} 5 & \mathrm{C} 4 & 117.3(3) \\ \mathrm{O} 3 & \mathrm{C} 5 & \mathrm{C} 6 & 121.8(4) \\ \mathrm{C} 6 & \mathrm{C} 5 & \mathrm{C} 4 & 120.9(4) \\ \mathrm{C} 7 & \mathrm{C} 6 & \mathrm{C} 5 & 119.9(4) \\ \mathrm{C} 8 & \mathrm{C} 7 & \mathrm{C} 6 & 120.1(4) \\ \mathrm{C} 7 & \mathrm{C} 8 & \mathrm{C} 9 & 119.6(4) \\ \mathrm{C} 4 & \mathrm{C} 9 & \mathrm{C} 8 & 121.8(4) \\ \mathrm{C} 3 & \mathrm{C} 10 & \mathrm{C} 11 & 109.8(4) \\ \mathrm{C} 12 & \mathrm{C} 11 & \mathrm{C} 10 & 118.6(6) \\ \mathrm{C} 11 & \mathrm{C} 12 & \mathrm{C} 13 & 113.0(8) \\ \mathrm{C} 14 & \mathrm{C} 13 & \mathrm{C} 12 & 105.0(10) \\ \mathrm{N} 1 & \mathrm{C} 15 & \mathrm{C} 16 & 117.3(3) \\ \mathrm{O} 4 & \mathrm{C} 15 & \mathrm{~N} 1 & 121.5(3) \\ \mathrm{O} 4 & \mathrm{C} 15 & \mathrm{C} 16 & 121.2(4)\end{array}$

Table S18 Torsion Angles for 3ao.
A B C D Angle ${ }^{\circ}$
A B C D Angle $/^{\circ}$
$\mathrm{N} 1 \mathrm{C} 2 \mathrm{C} 3 \quad \mathrm{C} 4$ 0.6(5)
C3 C10C11 C12-174.3(7)
$\mathrm{N} 1 \mathrm{C} 2 \mathrm{C} 3 \quad \mathrm{C} 10-177.2(4)$
C4 C3 C10C11 -77.1(5)
$\mathrm{O} 1 \mathrm{C} 1 \mathrm{C} 2 \mathrm{~N} 122.2(4)$
$\begin{array}{lllll}\mathrm{C} 4 & \mathrm{C} 5 & \mathrm{C} 6 & \mathrm{C} 7 & -1.5(5)\end{array}$
O1 C1 C2 C3 $-158.6(4)$
$\begin{array}{lllll}\mathrm{C} 5 & \mathrm{C} 4 & \mathrm{C} 9 & \mathrm{C} 8 & 0.2(5)\end{array}$
$\mathrm{O} 2 \mathrm{C} 1 \mathrm{C} 2 \mathrm{~N} 1-156.7(4)$
$\begin{array}{lllll}\mathrm{C} 5 & \mathrm{C} 6 & \mathrm{C} 7 & \mathrm{C} 8 & 0.4(6)\end{array}$
$\mathrm{O} 2 \mathrm{C} 1 \mathrm{C} 2 \mathrm{C} 322.5(6)$
$\begin{array}{lllll}\text { C6 } & \text { C7 } & \text { C8 } & \text { C9 } & 1.0(6)\end{array}$
O3 C5 C6 C7 179.9(3)
$\begin{array}{lllll}\mathrm{C} 7 & \mathrm{C} 8 & \mathrm{C} 9 & \mathrm{C} 4 & -1.3(6)\end{array}$
$\begin{array}{llll}\mathrm{C} 1 \mathrm{C} 2 \mathrm{C} 3 & \mathrm{C} 4 & -178.6(3)\end{array}$
$\begin{array}{lllll}\mathrm{C} 9 & \mathrm{C} 4 & \mathrm{C} 5 & \mathrm{O} 3 & 179.8(3)\end{array}$
$\mathrm{C} 1 \mathrm{C} 2 \mathrm{C} 3 \mathrm{C} 103.6(6)$
C9 $\mathrm{C} 4 \mathrm{C} 5 \mathrm{C} 6 \quad 1.1(5)$
$\mathrm{C} 2 \mathrm{~N} 1 \mathrm{C} 15 \mathrm{O} 4$ 4.8(6)
C10C3 C4 C5 113.8(4)
$\mathrm{C} 2 \mathrm{~N} 1 \mathrm{C} 15 \mathrm{C} 16-174.2(3)$
C10C3 C4 C9 -64.8(5)
C2 C3 C4 C5 -64.3(5)
C10C11 C12C13 171.6(7)
C2 C3 C4 C9 117.2(4)
C11 C12C13 C14-173.1(10)
C2 C3 C10C11 100.8(5)
C15N1 C2 C1 72.4(4)
$\mathrm{C} 3 \mathrm{C} 4 \mathrm{C} 5 \mathrm{O} 3 \quad 1.3(5)$
C15N1 C2 C3 $-106.9(4)$
C3 C4 C5 C6 $-177.4(3)$
$\begin{array}{llll}\mathrm{C} 17 \mathrm{O} 1 & \mathrm{C} 1 & \mathrm{O} 2 & 1.3(5)\end{array}$
$\begin{array}{llll}\mathrm{C} 3 \mathrm{C} 4 \mathrm{C} 9 & \mathrm{C} 8 & 178.8(3)\end{array}$
$\begin{array}{llll}\mathrm{C} 17 \mathrm{O} 1 & \mathrm{C} 1 & \mathrm{C} 2 & -177.6(3)\end{array}$

Table S19 Hydrogen Atom Coordinates $\left(\AA \times 10^{4}\right)$ and Isotropic Displacement Parameters $\left(\AA^{2} \times 10^{3}\right)$ for 3 ao.

$\begin{array}{lllll}\operatorname{Atom} x & y & z & \mathrm{U}(\mathrm{eq})\end{array}$ 


$\begin{array}{lllll}\text { H1 } & 1473 & 5050 & 1059 & 84 \\ \text { H3 } & 2006 & 3705 & 1596 & 129 \\ \text { H6 } & 1301 & 2757 & 1278 & 91 \\ \text { H7 } & 719 & 2208 & 413 & 105 \\ \text { H8 } & 793 & 2690 & -627 & 112 \\ \text { H9 } & 1474 & 3703 & -807 & 99 \\ \text { H10A 3039 } & 4829 & -622 & 121 \\ \text { H10B 2738 } & 4064 & -802 & 121 \\ \text { H11A 3560 } & 4310 & 370 & 200 \\ \text { H11B 3312 } & 3553 & 130 & 200 \\ \text { H12A 4287 } & 4357 & -503 & 241 \\ \text { H12B 4082 } & 3555 & -646 & 241 \\ \text { H13A 4731 } & 3988 & 557 & 279 \\ \text { H13B 4595 } & 3182 & 345 & 279 \\ \text { H14A 5316 } & 3304 & -505 & 464 \\ \text { H14B 5731 } & 3566 & 122 & 464 \\ \text { H14C 5403 } & 4129 & -367 & 464 \\ \text { H16A -191 } & 5619 & 762 & 133 \\ \text { H16B 326 } & 6114 & 1169 & 133 \\ \text { H16C 308 } & 5283 & 1303 & 133 \\ \text { H17A 3180 } & 6935 & 443 & 160 \\ \text { H17B 2593 } & 7293 & 890 & 160 \\ \text { H17C 2471 } & 7236 & 128 & \end{array}$

\section{Crystallographic data 5ta}

Table S20 Crystal data and structure refinement for 5 ta

$\begin{array}{ll}\text { Identification code } & \mathbf{5 t a} \\ \text { Empirical formula } & \mathrm{C}_{24} \mathrm{H}_{22} \mathrm{NO}_{2} \mathrm{Cl}_{3} \\ \text { Formula weight } & 462.77 \\ \text { Temperature/K } & 173.15 \\ \text { Crystal system } & \text { monoclinic } \\ \text { Space group } & \mathrm{P} 2{ }_{1} / \mathrm{c} \\ \mathrm{a} / \AA & 9.5912(8) \\ \mathrm{b} / \AA & 21.7969(19) \\ \mathrm{c} / \AA & 10.6004(9) \\ \alpha /{ }^{\circ} & 90 \\ \beta /{ }^{\circ} & 92.825(3) \\ \gamma /{ }^{\circ} & 90 \\ \text { Volume } / \AA^{3} & 2213.4(3)\end{array}$




$\begin{array}{ll}\mathrm{Z} & 4 \\ \rho_{\text {calc }} / \mathrm{cm}^{3} & 1.389 \\ \mu / \mathrm{mm}^{-1} & 0.435 \\ \mathrm{~F}(000) & 960.0 \\ \text { Crystal size } / \mathrm{mm}^{3} & 0.19 \times 0.16 \times 0.15 \\ \text { Radiation } & \mathrm{MoK} \alpha(\lambda=0.71073) \\ 2 \Theta \text { range for data collection } /{ }^{\circ} 4.276 \text { to } 50.012 \\ \text { Index ranges } & -9 \leq \mathrm{h} \leq 11,-24 \leq \mathrm{k} \leq 25,-12 \leq 1 \leq 12 \\ \text { Reflections collected } & 15894 \\ \text { Independent reflections } & 3911\left[\mathrm{R}_{\text {int }}=0.0486, \mathrm{R}_{\text {sigma }}=0.0427\right] \\ \text { Data/restraints/parameters } & 3911 / 0 / 273 \\ \text { Goodness-of-fit on } \mathrm{F}^{2} & 1.130 \\ \text { Final R indexes [I }>=2 \sigma(\mathrm{I})] & \mathrm{R}_{1}=0.0564, \mathrm{wR}_{2}=0.1417 \\ \text { Final R indexes [all data] } & \mathrm{R}_{1}=0.0725, \mathrm{wR}_{2}=0.1495 \\ \text { Largest diff. peak/hole } / \mathrm{e} \AA^{-3} 0.46 /-0.45\end{array}$

Table S21 Fractional Atomic Coordinates $\left(\times 10^{4}\right)$ and Equivalent Isotropic Displacement Parameters $\left(\AA^{2} \times 10^{3}\right)$ for 5 ta. $U_{\text {eq }}$ is defined as $1 / 3$ of of the trace of the orthogonalised $U_{\text {IJ }}$ tensor.

\begin{tabular}{lllll}
\multicolumn{2}{l}{ Atom $\boldsymbol{x}$} & $\boldsymbol{y}$ & $\boldsymbol{z}$ & $\mathbf{U}(\mathbf{e q})$ \\
$\mathrm{C} 1$ & $9354(3)$ & $3637.2(14)$ & $145(3)$ & $26.8(7)$ \\
$\mathrm{C} 2$ & $10218(3)$ & $3829.6(16)$ & $-791(3)$ & $32.6(7)$ \\
$\mathrm{C} 3$ & $11223(3)$ & $3421.6(18)$ & $-1174(3)$ & $40.3(8)$ \\
$\mathrm{C} 4$ & $11393(3)$ & $2851.0(17)$ & $-615(3)$ & $41.5(9)$ \\
$\mathrm{C} 5$ & $10559(3)$ & $2668.1(16)$ & $364(3)$ & $35.7(8)$ \\
$\mathrm{C} 6$ & $9527(3)$ & $3062.8(14)$ & $721(3)$ & $28.2(7)$ \\
$\mathrm{C} 7$ & $8438(3)$ & $3020.0(14)$ & $1709(3)$ & $27.6(7)$ \\
$\mathrm{C} 8$ & $7235(3)$ & $3375.1(13)$ & $1009(3)$ & $26.3(7)$ \\
$\mathrm{C} 9$ & $8137(3)$ & $3947.3(14)$ & $752(3)$ & $27.3(7)$ \\
$\mathrm{C} 10$ & $8638(3)$ & $4128.6(13)$ & $2108(3)$ & $26.0(7)$ \\
$\mathrm{C} 11$ & $8915(3)$ & $3482.7(13)$ & $2769(3)$ & $24.7(6)$ \\
$\mathrm{C} 12$ & $8203(3)$ & $3437.3(13)$ & $4001(3)$ & $24.6(6)$ \\
$\mathrm{C} 13$ & $6959(3)$ & $3148.7(13)$ & $4163(3)$ & $26.4(7)$ \\
$\mathrm{C} 14$ & $6265(3)$ & $3178.8(13)$ & $5314(3)$ & $26.8(7)$ \\
$\mathrm{C} 15$ & $4961(3)$ & $2891.9(14)$ & $5482(3)$ & $31.2(7)$ \\
$\mathrm{C} 16$ & $4316(3)$ & $2934.8(15)$ & $6602(3)$ & $35.4(8)$ \\
$\mathrm{C} 17$ & $4941(4)$ & $3270.3(15)$ & $7607(3)$ & $36.8(8)$ \\
$\mathrm{C} 18$ & $6200(3)$ & $3551.8(15)$ & $7483(3)$ & $33.4(7)$ \\
$\mathrm{C} 19$ & $6898(3)$ & $3515.0(13)$ & $6344(3)$ & $26.9(7)$ \\
& & & &
\end{tabular}




$\begin{array}{lllll}\mathrm{C} 20 & 8212(3) & 3795.4(13) & 6181(3) & 28.3(7) \\ \mathrm{C} 21 & 8836(3) & 3760.7(13) & 5058(3) & 27.0(7) \\ \mathrm{C} 22 & 6636(4) & 5291.9(16) & 3974(4) & 43.8(9) \\ \mathrm{C} 23 & 7813(3) & 5032.6(13) & 3268(3) & 27.5(7) \\ \mathrm{C} 24 & 2925(3) & 4147.7(16) & 1604(4) & 40.9(9) \\ \mathrm{C} 11 & 2243.2(10) & 4815.1(4) & 2253.4(11) & 58.8(3) \\ \mathrm{C} 12 & 4097.1(11) & 4330.1(6) & 442.5(11) & 63.9(3) \\ \mathrm{C} 13 & 3751.7(11) & 3708.9(5) & 2818.6(11) & 60.0(3) \\ \mathrm{N} 1 & 7580(2) & 4488.4(11) & 2712(2) & 27.5(6) \\ \mathrm{O} 1 & 8933(2) & 5307.8(10) & 3196(2) & 34.8(5) \\ \mathrm{O} 2 & 10094(2) & 4018.0(11) & 4849(2) & 35.7(5)\end{array}$

Table S22 Anisotropic Displacement Parameters $\left(\AA^{2} \times 10^{3}\right)$ for 5ta. The Anisotropic displacement factor exponent takes the form: $-2 \pi^{2}\left[h^{2} a * 2 U_{11}+2 h k a * b * U_{12}+\ldots\right]$.

$\begin{array}{lllllll}\text { Atom } \mathbf{U}_{11} & \mathbf{U}_{22} & \mathbf{U}_{33} & \mathbf{U}_{23} & \mathbf{U}_{13} & \mathbf{U}_{\mathbf{1 2}} \\ \mathrm{C} 1 & 24.4(15) & 29.1(17) & 26.1(16) & -7.5(13) & -5.3(12) & -2.5(12) \\ \mathrm{C} 2 & 26.4(16) & 38.9(19) & 31.8(18) & -1.4(15) & -5.5(13) & -4.7(13) \\ \mathrm{C} 3 & 28.0(17) & 57(2) & 35(2) & -6.2(17) & -0.5(14) & -1.9(15) \\ \mathrm{C} 4 & 26.5(17) & 53(2) & 45(2) & -13.8(18) & 0.1(15) & 7.9(15) \\ \mathrm{C} 5 & 28.9(17) & 35.5(19) & 42(2) & -9.6(15) & -8.2(14) & 3.3(13) \\ \mathrm{C} 6 & 24.0(15) & 29.1(17) & 30.5(17) & -8.0(13) & -8.1(12) & -2.1(12) \\ \mathrm{C} 7 & 27.4(16) & 21.3(15) & 33.6(18) & -5.0(13) & -4.7(13) & -2.8(12) \\ \mathrm{C} 8 & 22.2(15) & 27.8(16) & 28.3(17) & -6.4(13) & -4.4(12) & -1.2(12) \\ \mathrm{C} 9 & 22.7(15) & 25.6(16) & 33.2(17) & -2.0(13) & -4.2(12) & -0.5(11) \\ \mathrm{C} 10 & 21.6(15) & 21.5(15) & 34.8(18) & -1.4(13) & 0.6(12) & -3.2(11) \\ \mathrm{C} 11 & 20.2(15) & 24.1(15) & 29.3(16) & -1.6(13) & -2.8(12) & -2.7(11) \\ \mathrm{C} 12 & 27.1(15) & 20.2(15) & 26.0(16) & 0.5(12) & -5.2(12) & 1.5(11) \\ \mathrm{C} 13 & 26.1(15) & 20.4(15) & 31.9(17) & -1.6(13) & -5.4(12) & 0.4(11) \\ \mathrm{C} 14 & 27.1(16) & 19.8(15) & 32.7(17) & 3.0(13) & -6.3(13) & 2.0(11) \\ \mathrm{C} 15 & 28.2(16) & 27.1(17) & 37.8(19) & 1.5(14) & -3.2(14) & -0.4(12) \\ \mathrm{C} 16 & 29.8(17) & 30.7(18) & 46(2) & 6.8(15) & 3.0(15) & -0.7(13) \\ \mathrm{C} 17 & 41.8(19) & 36.7(19) & 32.1(18) & 7.4(15) & 4.1(15) & 6.7(15) \\ \mathrm{C} 18 & 39.3(19) & 31.7(18) & 28.6(17) & 1.8(14) & -3.9(14) & 3.3(14) \\ \mathrm{C} 19 & 30.7(16) & 20.9(15) & 28.2(16) & 4.5(13) & -6.1(13) & 4.8(12) \\ \mathrm{C} 20 & 31.0(16) & 23.5(16) & 29.4(17) & -2.2(13) & -9.1(13) & 0.8(12) \\ \mathrm{C} 21 & 24.4(15) & 22.8(16) & 33.1(17) & -2.3(13) & -5.7(12) & -1.5(11) \\ \mathrm{C} 22 & 47(2) & 31.9(19) & 53(2) & -6.9(17) & 8.3(17) & 3.4(15) \\ \mathrm{C} 23 & 34.8(17) & 21.2(16) & 26.0(16) & 2.4(13) & -3.8(13) & -1.0(12) \\ \mathrm{C} 24 & 30.0(18) & 37(2) & 55(2) & 0.5(17) & -4.1(16) & 0.4(14) \\ \mathrm{C} 11 & 50.1(6) & 40.8(6) & 85.7(8) & -2.0(5) & 5.2(5) & 16.2(4)\end{array}$




$\begin{array}{lllllll}\mathrm{C} 12 & 50.1(6) & 79.8(8) & 62.9(7) & 9.9(6) & 11.9(5) & 4.0(5) \\ \mathrm{C} 13 & 62.1(7) & 53.0(6) & 64.7(7) & 11.0(5) & 0.5(5) & 23.6(5) \\ \mathrm{N} 1 & 23.0(13) & 20.9(13) & 38.5(15) & -3.5(11) & -0.8(11) & -4.8(10) \\ \mathrm{O} 1 & 38.6(13) & 26.5(12) & 39.1(13) & -9.2(10) & 1.3(10) & -10.8(9) \\ \mathrm{O} 2 & 30.3(12) & 39.7(14) & 36.5(13) & -7.6(11) & -4.2(10) & -12.7(9)\end{array}$

Table S23 Bond Lengths for 5ta.

\begin{tabular}{lllllll}
\multicolumn{2}{c}{ Atom Atom Length/Å } & \multicolumn{3}{c}{ Atom Atom Length/Å } \\
$\mathrm{C} 1$ & $\mathrm{C} 2$ & $1.388(4)$ & $\mathrm{C} 13$ & $\mathrm{C} 14$ & $1.420(4)$ \\
$\mathrm{C} 1$ & $\mathrm{C} 6$ & $1.399(4)$ & $\mathrm{C} 14$ & $\mathrm{C} 15$ & $1.417(4)$ \\
$\mathrm{C} 1$ & $\mathrm{C} 9$ & $1.519(4)$ & $\mathrm{C} 14$ & $\mathrm{C} 19$ & $1.425(4)$ \\
$\mathrm{C} 2$ & $\mathrm{C} 3$ & $1.387(5)$ & $\mathrm{C} 15$ & $\mathrm{C} 16$ & $1.369(5)$ \\
$\mathrm{C} 3$ & $\mathrm{C} 4$ & $1.384(5)$ & $\mathrm{C} 16$ & $\mathrm{C} 17$ & $1.402(5)$ \\
$\mathrm{C} 4$ & $\mathrm{C} 5$ & $1.399(5)$ & $\mathrm{C} 17$ & $\mathrm{C} 18$ & $1.367(5)$ \\
$\mathrm{C} 5$ & $\mathrm{C} 6$ & $1.378(4)$ & $\mathrm{C} 18$ & $\mathrm{C} 19$ & $1.412(4)$ \\
$\mathrm{C} 6$ & $\mathrm{C} 7$ & $1.518(4)$ & $\mathrm{C} 19$ & $\mathrm{C} 20$ & $1.419(4)$ \\
$\mathrm{C} 7$ & $\mathrm{C} 8$ & $1.548(4)$ & $\mathrm{C} 20$ & $\mathrm{C} 21$ & $1.360(4)$ \\
$\mathrm{C} 7$ & $\mathrm{C} 11$ & $1.562(4)$ & $\mathrm{C} 21$ & $\mathrm{O} 2$ & $1.359(4)$ \\
$\mathrm{C} 8$ & $\mathrm{C} 9$ & $1.550(4)$ & $\mathrm{C} 22$ & $\mathrm{C} 23$ & $1.496(5)$ \\
$\mathrm{C} 9$ & $\mathrm{C} 10$ & $1.545(4)$ & $\mathrm{C} 23$ & $\mathrm{~N} 1$ & $1.338(4)$ \\
$\mathrm{C} 10$ & $\mathrm{C} 11$ & $1.589(4)$ & $\mathrm{C} 23$ & $\mathrm{O} 1$ & $1.236(4)$ \\
$\mathrm{C} 10$ & $\mathrm{~N} 1$ & $1.456(4)$ & $\mathrm{C} 24$ & $\mathrm{C} 11$ & $1.750(4)$ \\
$\mathrm{C} 11$ & $\mathrm{C} 12$ & $1.507(4)$ & $\mathrm{C} 24$ & $\mathrm{C} 12$ & $1.754(4)$ \\
$\mathrm{C} 12$ & $\mathrm{C} 13$ & $1.367(4)$ & $\mathrm{C} 24$ & $\mathrm{C} 13$ & $1.761(4)$ \\
$\mathrm{C} 12$ & $\mathrm{C} 21$ & $1.433(4)$ & & &
\end{tabular}

Table S24 Bond Angles for 5ta.

\begin{tabular}{|c|c|c|c|}
\hline \multicolumn{4}{|c|}{ Atom Atom Atom Angle $/^{\circ}$} \\
\hline $\mathrm{C} 2$ & $\mathrm{C} 1$ & $\mathrm{C} 6$ & $121.3(3)$ \\
\hline $\mathrm{C} 2$ & $\mathrm{C} 1$ & C9 & $131.8(3)$ \\
\hline C6 & $\mathrm{C} 1$ & C9 & $106.9(3)$ \\
\hline $\mathrm{C} 3$ & $\mathrm{C} 2$ & $\mathrm{C} 1$ & $117.6(3)$ \\
\hline $\mathrm{C} 4$ & $\mathrm{C} 3$ & $\mathrm{C} 2$ & $121.3(3)$ \\
\hline $\mathrm{C} 3$ & $\mathrm{C} 4$ & $\mathrm{C} 5$ & $120.9(3)$ \\
\hline C6 & $\mathrm{C} 5$ & $\mathrm{C} 4$ & $118.1(3)$ \\
\hline $\mathrm{C} 1$ & C6 & $\mathrm{C} 7$ & $106.6(3)$ \\
\hline $\mathrm{C} 5$ & C6 & $\mathrm{C} 1$ & $120.7(3)$ \\
\hline $\mathrm{C} 5$ & C6 & $\mathrm{C} 7$ & $132.8(3)$ \\
\hline C6 & C7 & $\mathrm{C} 8$ & $99.3(2)$ \\
\hline C6 & $\mathrm{C} 7$ & $\mathrm{C} 11$ & $105.6(2)$ \\
\hline
\end{tabular}

\begin{tabular}{|c|c|c|c|}
\hline \multicolumn{4}{|c|}{ Atom Atom Atom Angle ${ }^{\circ}$} \\
\hline $\mathrm{C} 13$ & $\mathrm{C} 12$ & $\mathrm{C} 21$ & $117.8(3)$ \\
\hline $\mathrm{C} 21$ & $\mathrm{C} 12$ & $\mathrm{C} 11$ & $116.9(3)$ \\
\hline $\mathrm{C} 12$ & $\mathrm{C} 13$ & $\mathrm{C} 14$ & $122.4(3)$ \\
\hline $\mathrm{C} 13$ & $\mathrm{C} 14$ & C19 & $118.8(3)$ \\
\hline $\mathrm{C} 15$ & $\mathrm{C} 14$ & $\mathrm{C} 13$ & 122.8 \\
\hline 15 & $\mathrm{C} 1$ & $\mathrm{C} 19$ & $118.4(3)$ \\
\hline $\mathrm{C} 16$ & $\mathrm{C} 15$ & $\mathrm{C} 14$ & $121.2(3)$ \\
\hline $\mathrm{C} 15$ & $\mathrm{C} 16$ & $\mathrm{C} 17$ & $120.0(3)$ \\
\hline $\mathrm{C} 18$ & $\mathrm{C} 17$ & $\mathrm{C} 16$ & $120.5(3)$ \\
\hline 17 & $\mathrm{C} 18$ & $\mathrm{C} 19$ & $121.0(3)$ \\
\hline 18 & $\mathrm{C} 19$ & $\mathrm{C} 14$ & $118.8(3)$ \\
\hline $\mathrm{C} 18$ & $\mathrm{C} 19$ & $\mathrm{C} 20$ & $122.7(3)$ \\
\hline
\end{tabular}




$\begin{array}{llllllll}\mathrm{C} 8 & \mathrm{C} 7 & \mathrm{C} 11 & 101.9(2) & \mathrm{C} 20 & \mathrm{C} 19 & \mathrm{C} 14 & 118.4(3) \\ \mathrm{C} 7 & \mathrm{C} 8 & \mathrm{C} 9 & 94.4(2) & \mathrm{C} 21 & \mathrm{C} 20 & \mathrm{C} 19 & 120.9(3) \\ \mathrm{C} 1 & \mathrm{C} 9 & \mathrm{C} 8 & 99.4(2) & \mathrm{C} 20 & \mathrm{C} 21 & \mathrm{C} 12 & 121.6(3) \\ \mathrm{C} 1 & \mathrm{C} 9 & \mathrm{C} 10 & 107.3(2) & \mathrm{C} 20 & \mathrm{C} 21 & \mathrm{O} 2 & 123.5(3) \\ \mathrm{C} 10 & \mathrm{C} 9 & \mathrm{C} 8 & 101.1(2) & \mathrm{O} 2 & \mathrm{C} 21 & \mathrm{C} 12 & 114.9(3) \\ \mathrm{C} 9 & \mathrm{C} 10 & \mathrm{C} 11 & 102.8(2) & \mathrm{N} 1 & \mathrm{C} 23 & \mathrm{C} 22 & 116.2(3) \\ \mathrm{N} 1 & \mathrm{C} 10 & \mathrm{C} 9 & 110.8(2) & \mathrm{O} 1 & \mathrm{C} 23 & \mathrm{C} 22 & 121.8(3) \\ \mathrm{N} 1 & \mathrm{C} 10 & \mathrm{C} 11 & 112.9(2) & \mathrm{O} 1 & \mathrm{C} 23 & \mathrm{~N} 1 & 122.0(3) \\ \mathrm{C} 7 & \mathrm{C} 11 & \mathrm{C} 10 & 102.6(2) & \mathrm{C} 1 & \mathrm{C} 24 & \mathrm{C} 13 & 109.1(2) \\ \mathrm{C} 12 & \mathrm{C} 11 & \mathrm{C} 7 & 116.9(2) & \mathrm{C} 2 & \mathrm{C} 24 & \mathrm{C} 11 & 110.6(2) \\ \mathrm{C} 12 & \mathrm{C} 11 & \mathrm{C} 10 & 111.5(2) & \mathrm{C} 2 & \mathrm{C} 24 & \mathrm{C} 13 & 110.81(19) \\ \mathrm{C} 13 & \mathrm{C} 12 & \mathrm{C} 11 & 125.1(3) & \mathrm{C} 23 & \mathrm{~N} 1 & \mathrm{C} 10 & 124.5(2)\end{array}$

Table S25 Hydrogen Atom Coordinates $\left(\AA \times 10^{4}\right)$ and Isotropic Displacement Parameters $\left(\AA^{2} \times 10^{3}\right)$ for 5 ta.

\begin{tabular}{lllll}
\multicolumn{2}{l}{ Atom $\boldsymbol{x}$} & $\boldsymbol{y}$ & $\boldsymbol{z}$ & $\mathbf{U}(\mathbf{e q})$ \\
H2 & 10124.93 & 4226.55 & -1155.14 & 39 \\
H3 & 11805.61 & 3535.94 & -1834.32 & 48 \\
H4 & 12086.25 & 2579.65 & -899.44 & 50 \\
H5 & 10700.21 & 2283.12 & 770.72 & 43 \\
H7 & 8207.94 & 2596.68 & 1996.64 & 33 \\
H8A & 6453.04 & 3465.71 & 1555.14 & 32 \\
H8B & 6885.54 & 3168.05 & 224.7 & 32 \\
H9 & 7660.56 & 4278.66 & 240.72 & 33 \\
H10 & 9528.31 & 4366.51 & 2091.68 & 31 \\
H11 & 9943.64 & 3434.5 & 2944.34 & 30 \\
H13 & 6543.38 & 2919.79 & 3480.81 & 32 \\
H15 & 4526.79 & 2665.73 & 4804.27 & 37 \\
H16 & 3445.37 & 2737.15 & 6698.15 & 42 \\
H17 & 4485.02 & 3302.24 & 8379.88 & 44 \\
H18 & 6611.98 & 3775.71 & 8174.15 & 40 \\
H20 & 8662.91 & 4010.38 & 6865.47 & 34 \\
H22A 6875.38 & 5277.35 & 4882.78 & 66 \\
H22B & 5789.45 & 5049.88 & 3786.81 & 66 \\
H22C 6470.88 & 5718.36 & 3714.42 & 66 \\
H24 & 2138.73 & 3902.65 & 1206.74 & 49 \\
H1 & 6728.94 & 4337.74 & 2712.12 & 33 \\
H2A & 10395.01 & 4201.79 & 5503.92 & 54
\end{tabular}




\section{Literature}

[1] G. Liu, Y. Shen, Z. Zhou, X. Lu, Angew. Chem. Int. Ed. 2013, 52, 6033; H. M. Petrassi, B. K. Sharpless, J. W. Kelly, Org. Lett. 2001, 3, 139.

[2] W. A. Wasylenko, N. Kebede, B. M. Showalter, N. Matsunaga, A. P. Miceli, Y. Liu, L. R. Ryzhkov, C. M. Hadad, J. P. Toscano, J. Am. Chem. Soc. 2006, 128, 13142. B. Li, J. Lan, D. Wu, J. You, Angew. Chem. Int. Ed. 2015, 54, 14008.

[3] S. Cai, K. Yang, D. Z. Wang, Org. Lett. 2014, 16, 2606.

[4] J. M. Medina, J. H. Ko, H. D. Maynard, N. K. Garg, Macromolecules 2017, 50, 580.

[5] M. J. Koh, R. K. M. Khan, S. Torker, A. H. Hoveyda, Angew. Chem. Int. Ed. 2014, 53, 1968.

[6] Q. Yang, P. Y. Choy, B. Fan, F. Y. Kwong, Adv. Synth. Cat. 2015, 357, 2345

[7] M. Lautens, K. Fagnou, V. Zunic, Org. Lett. 2002, 4, 3465.

[8] J. A. Kaydos, D. L. Smith, J. Org. Chem. 1983, 48, 1096. 


\section{NMR spectra}

\section{${ }^{1} \mathrm{H}$ and ${ }^{13} \mathrm{C}$ NMR of $\mathbf{3 a a}$}

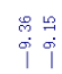

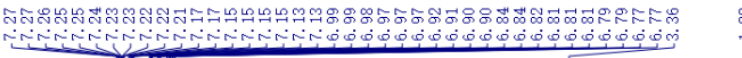<smiles>CC(=O)NC(C(C)=O)=C(c1ccccc1)c1ccccc1O</smiles>
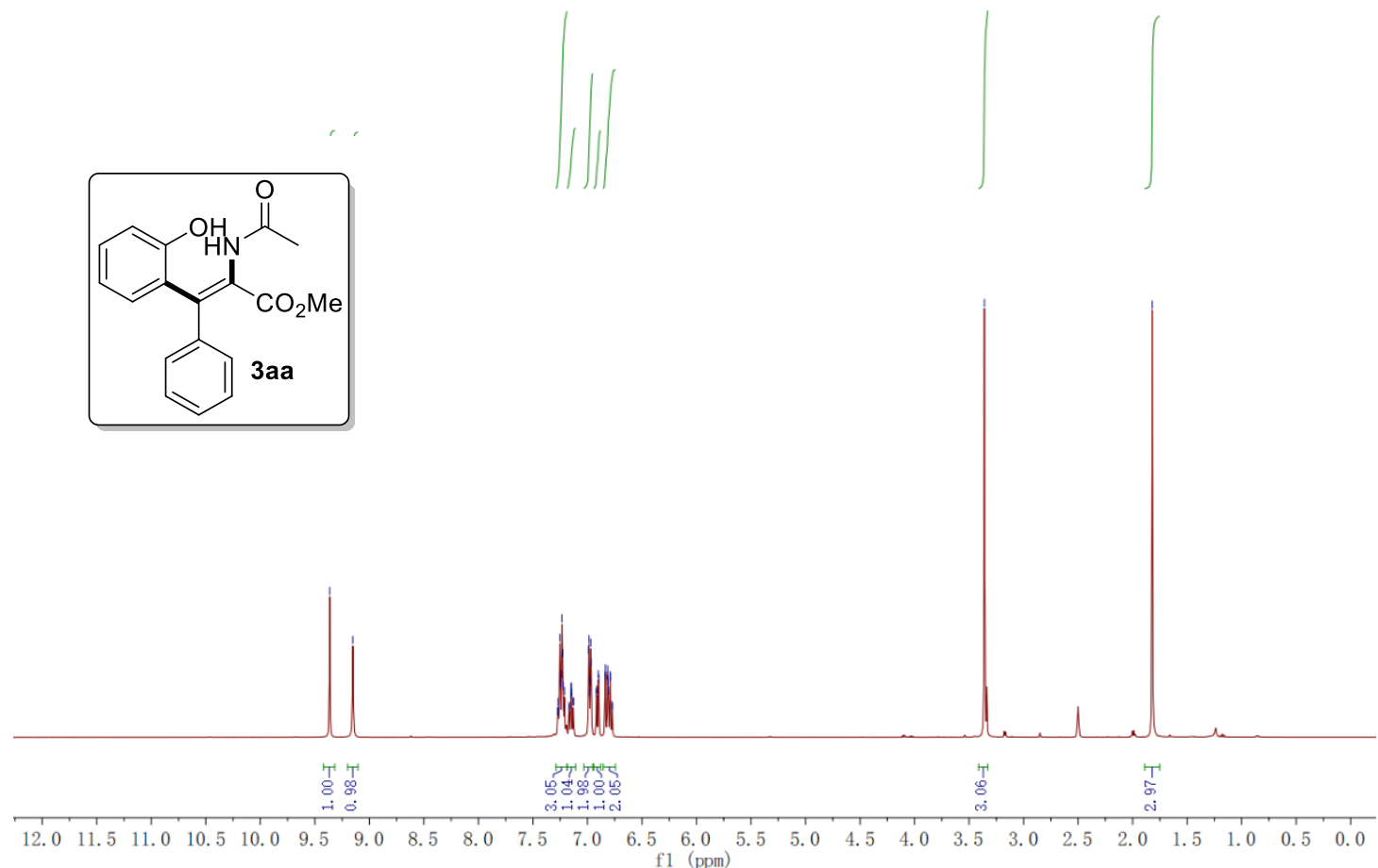

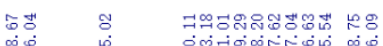

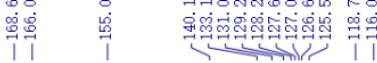

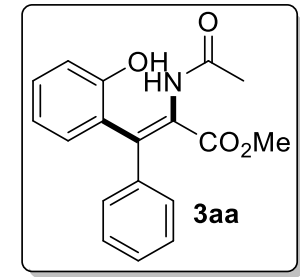

200

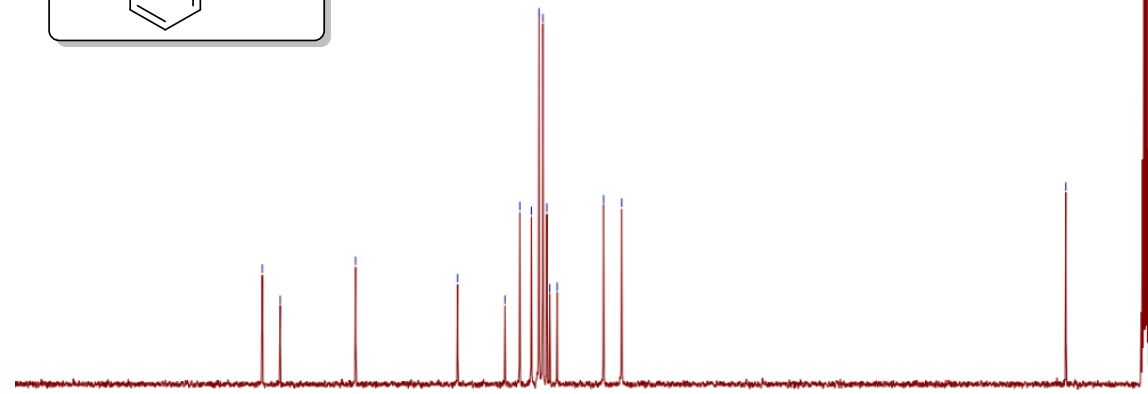


${ }^{1} \mathrm{H}$ and ${ }^{13} \mathrm{C}$ NMR of $\mathbf{3 b a}$

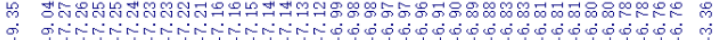
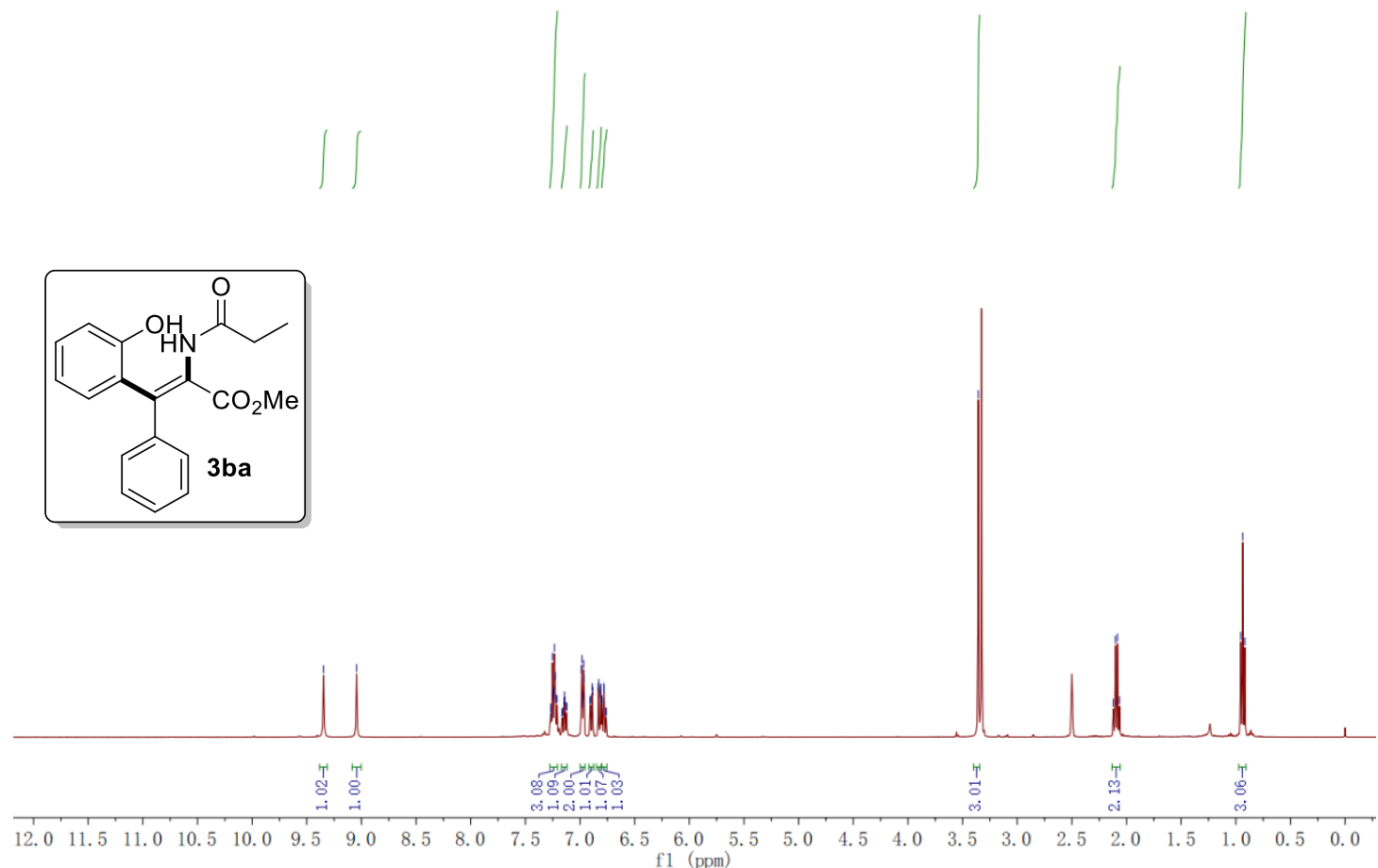

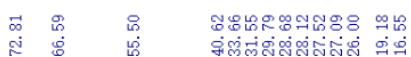

।
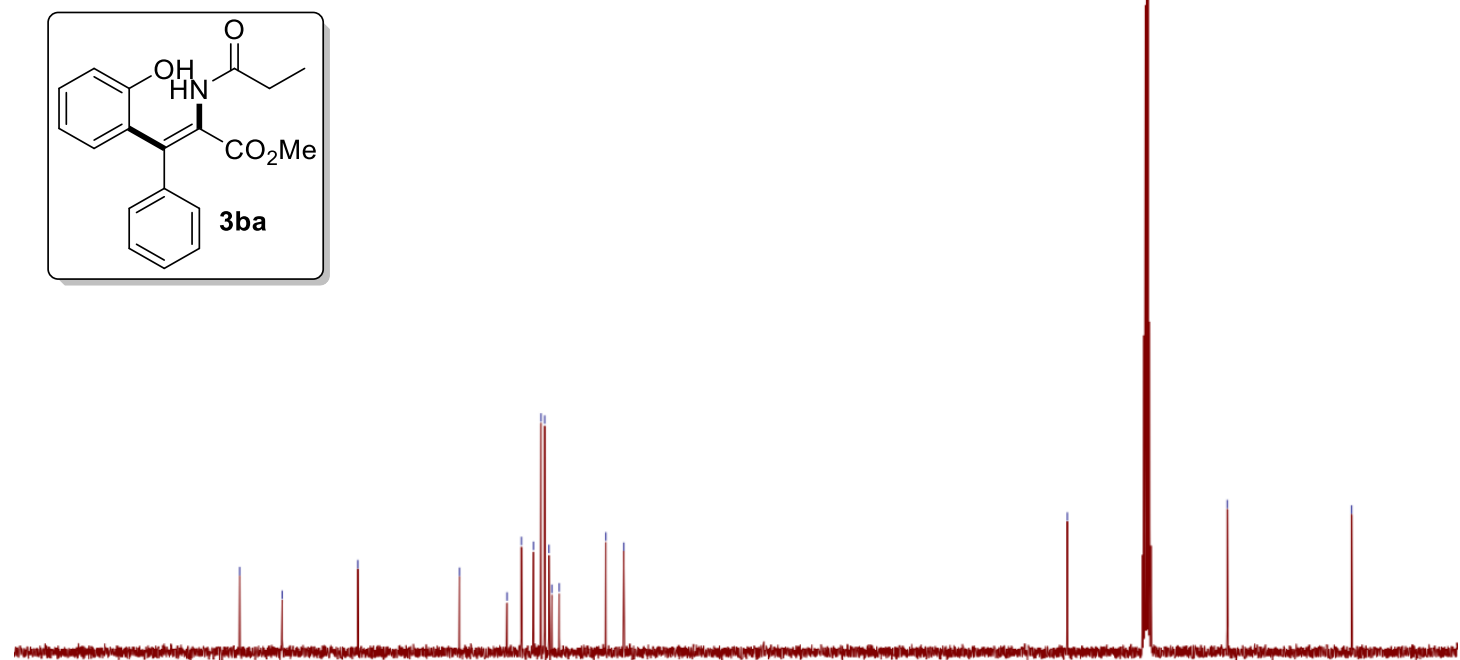

$\begin{array}{llllllllllllllllllllllll}200 & 190 & 180 & 170 & 160 & 150 & 140 & 130 & 120 & 110 & 100 & 90 & 80 & 70 & 60 & 50 & 40 & 30 & 20 & 10 & 0\end{array}$ 
${ }^{1} \mathrm{H}$ and ${ }^{13} \mathrm{C}$ NMR of $\mathbf{3 c a}$

m

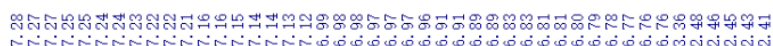

혀
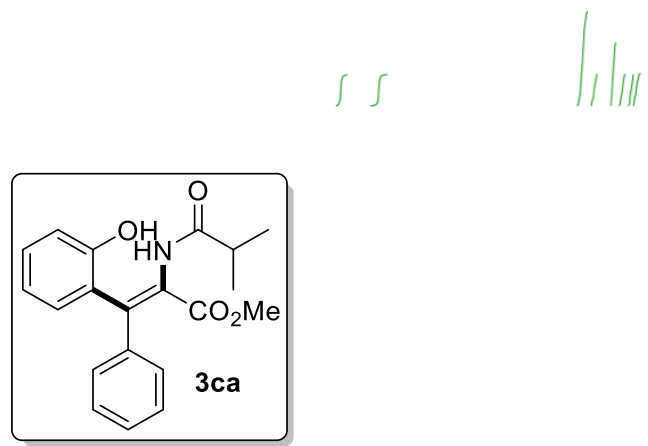

$\iint$
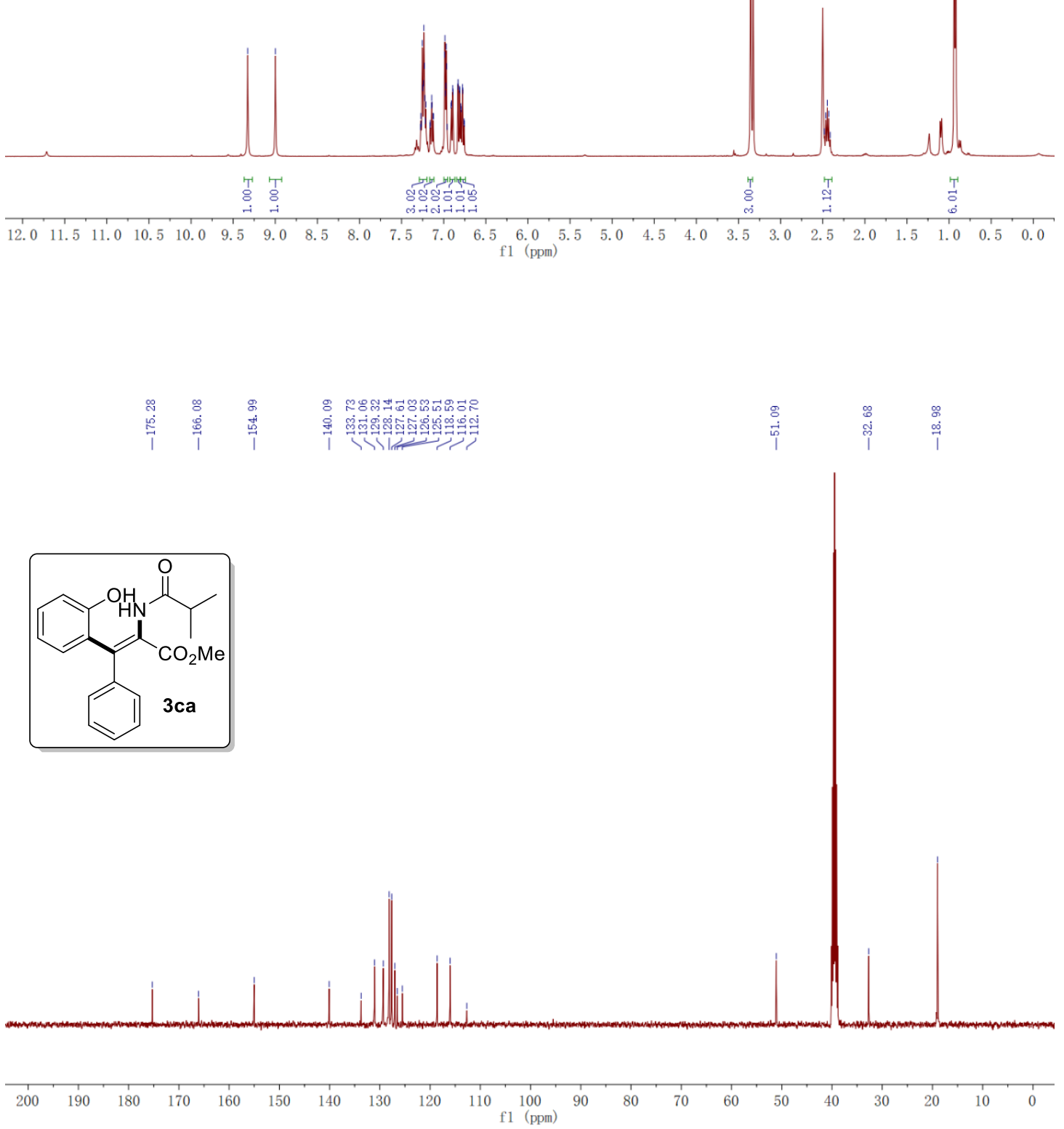

57 
${ }^{1} \mathrm{H}$ and ${ }^{13} \mathrm{C}$ NMR of $\mathbf{3 d a}$

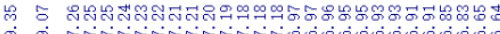

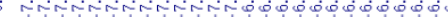

范
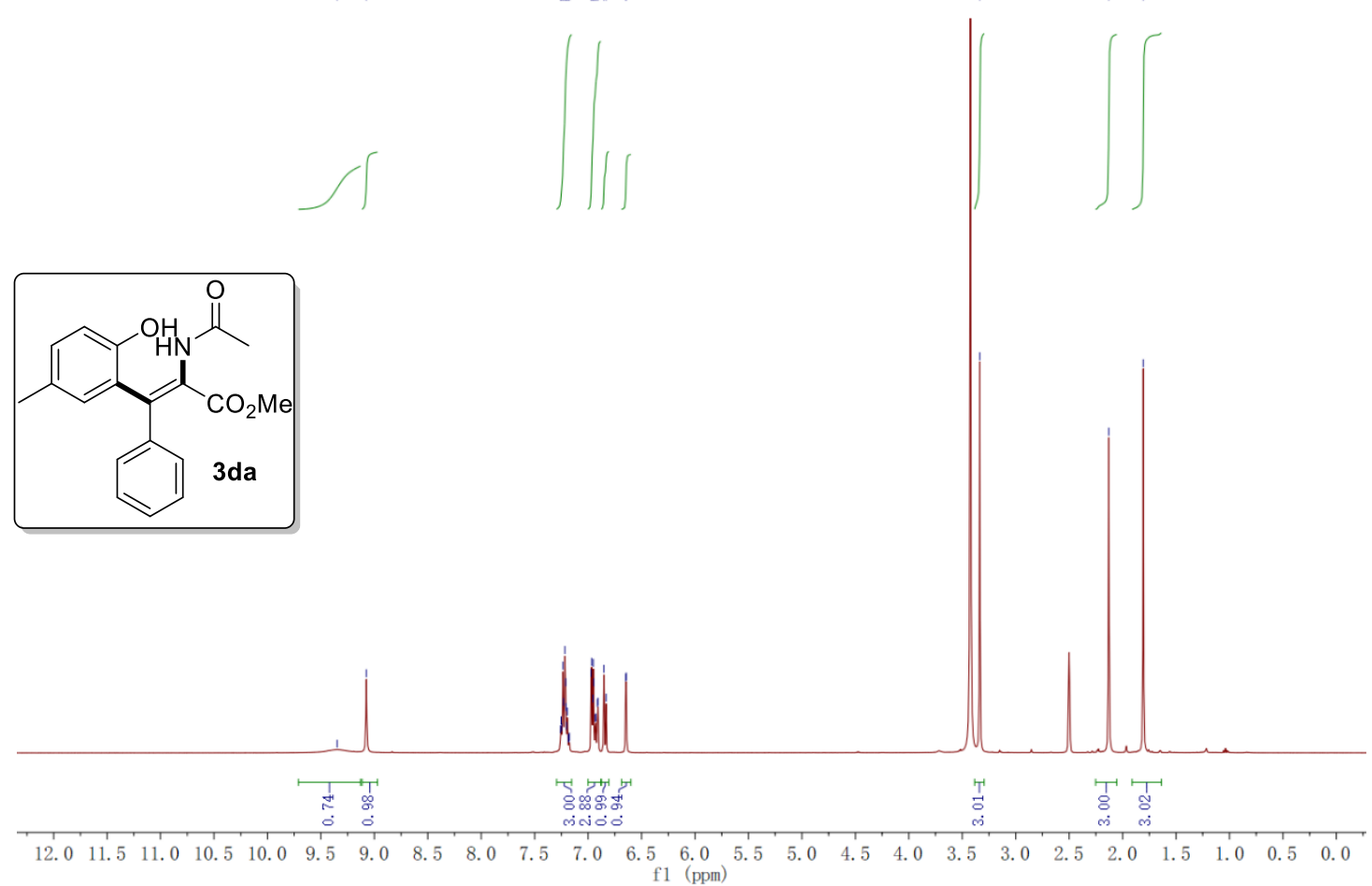

१ं

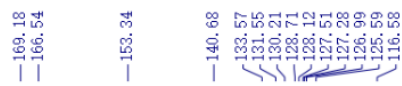

8
0
0
0
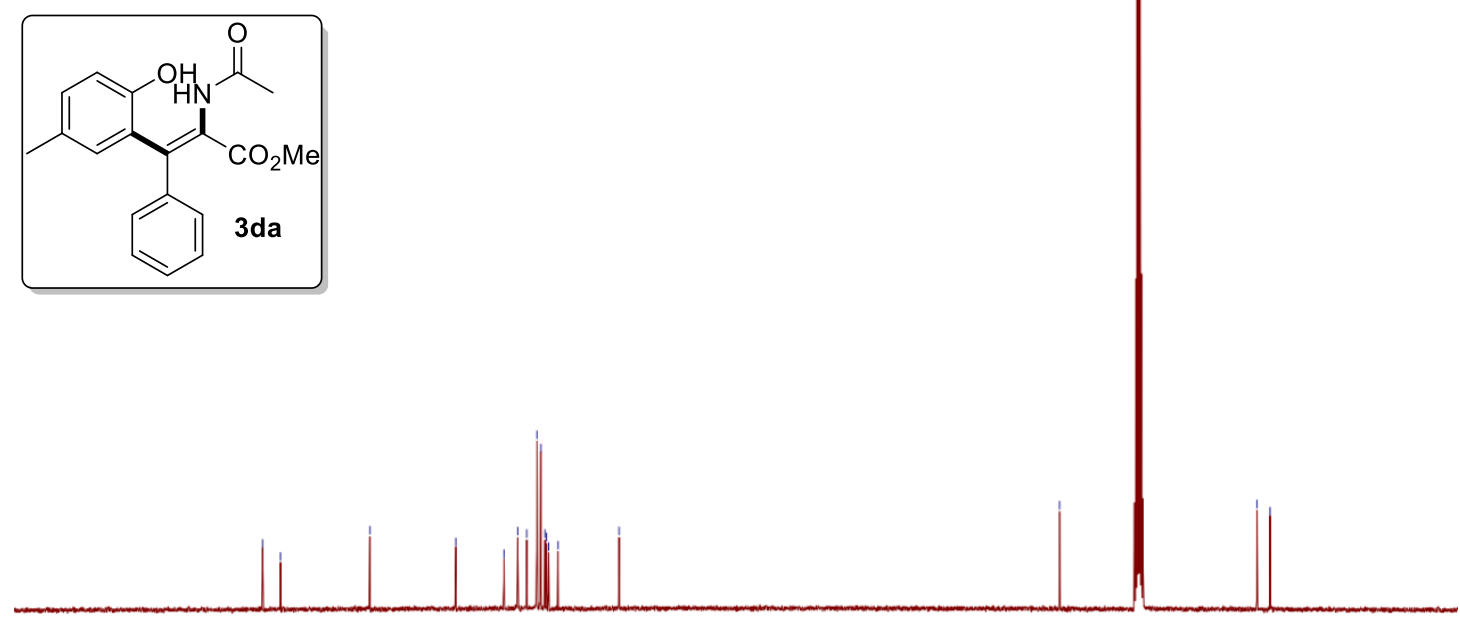

200

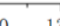

$\begin{array}{llll}110 & 100 & 90\end{array}$

11 (ppm) 
${ }^{1} \mathrm{H}$ and ${ }^{13} \mathrm{C}$ NMR of 3 ea

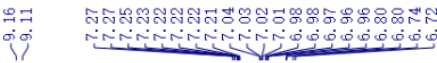

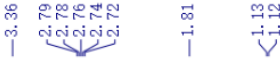

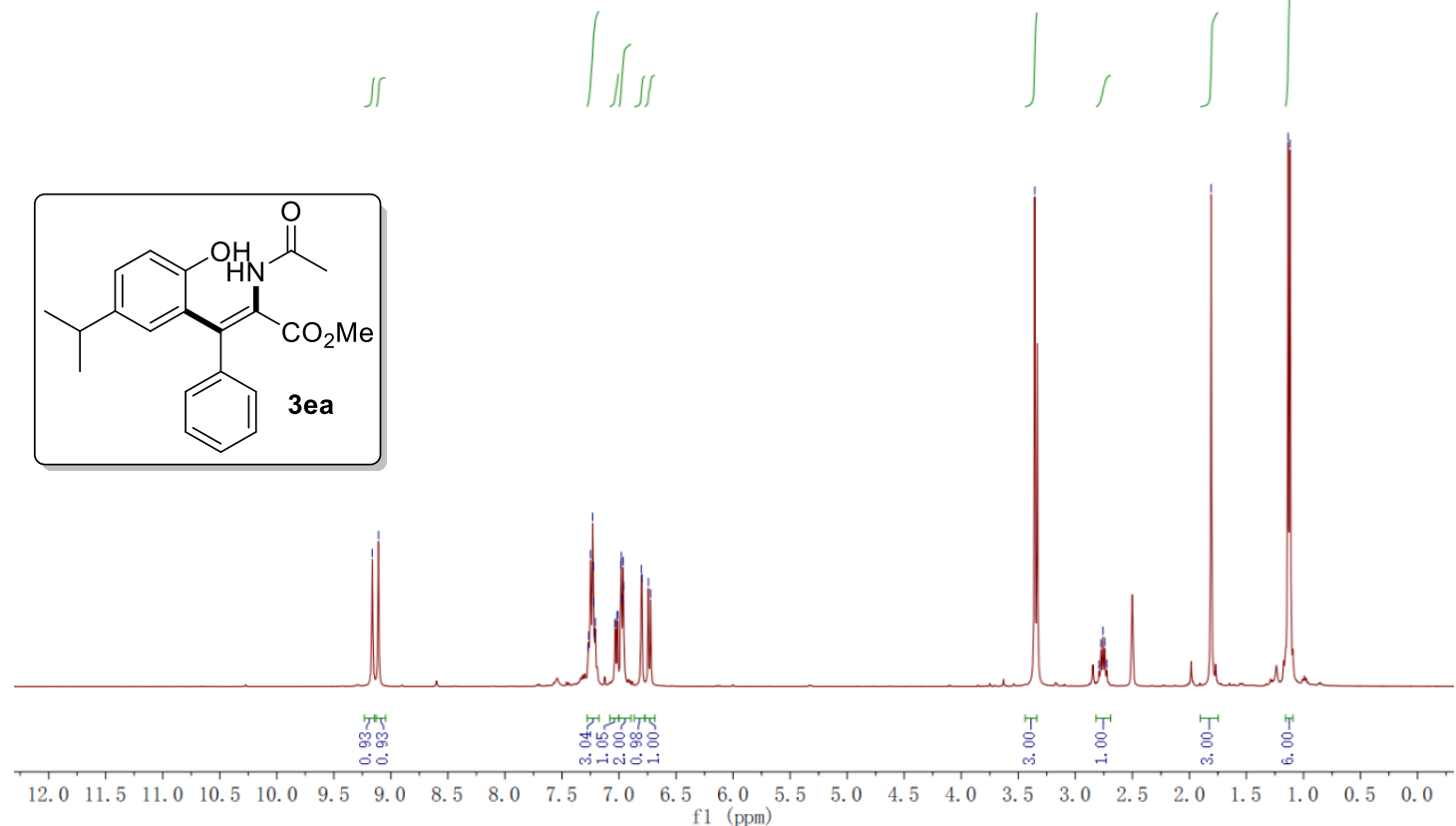

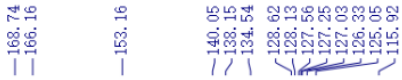

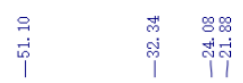
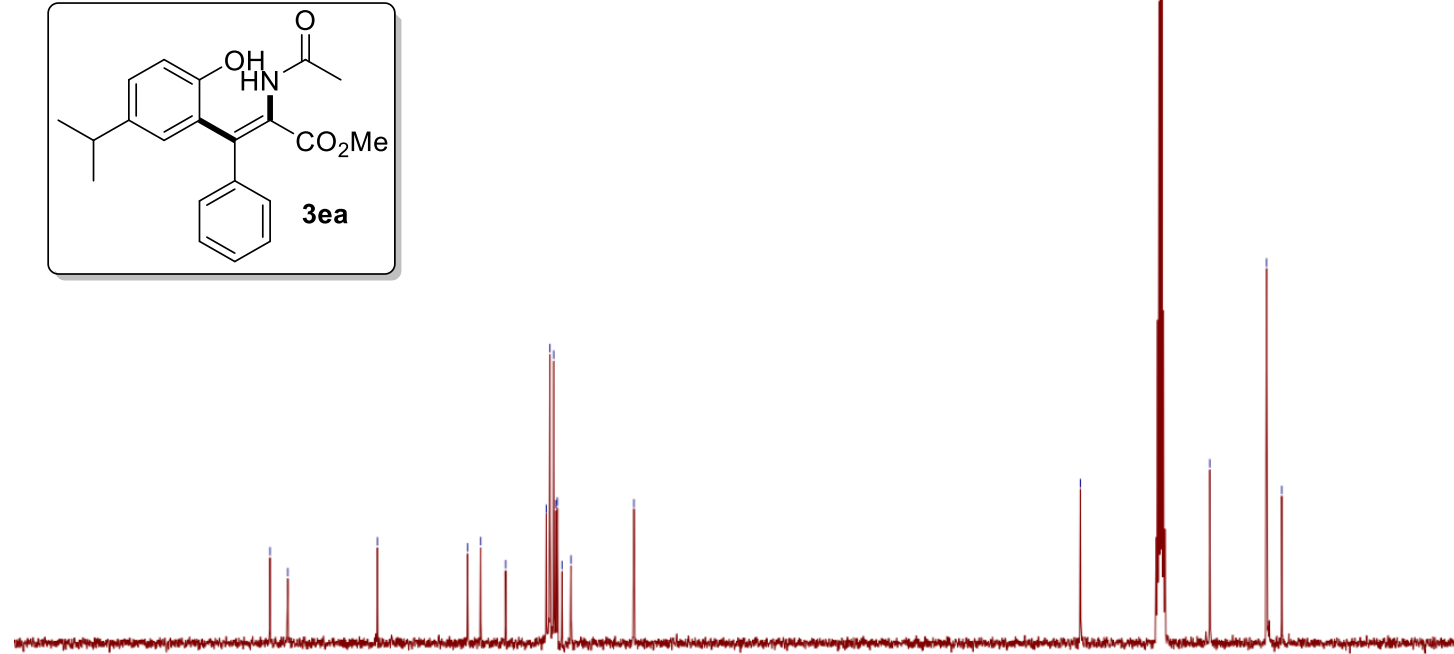

200

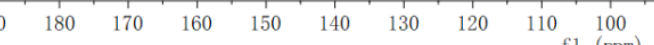

90

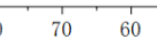

50

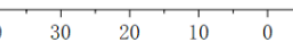


${ }^{1} \mathrm{H}$ and ${ }^{13} \mathrm{C}$ NMR of $\mathbf{3 f a}$

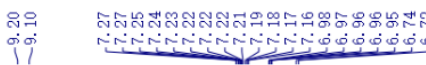

J5
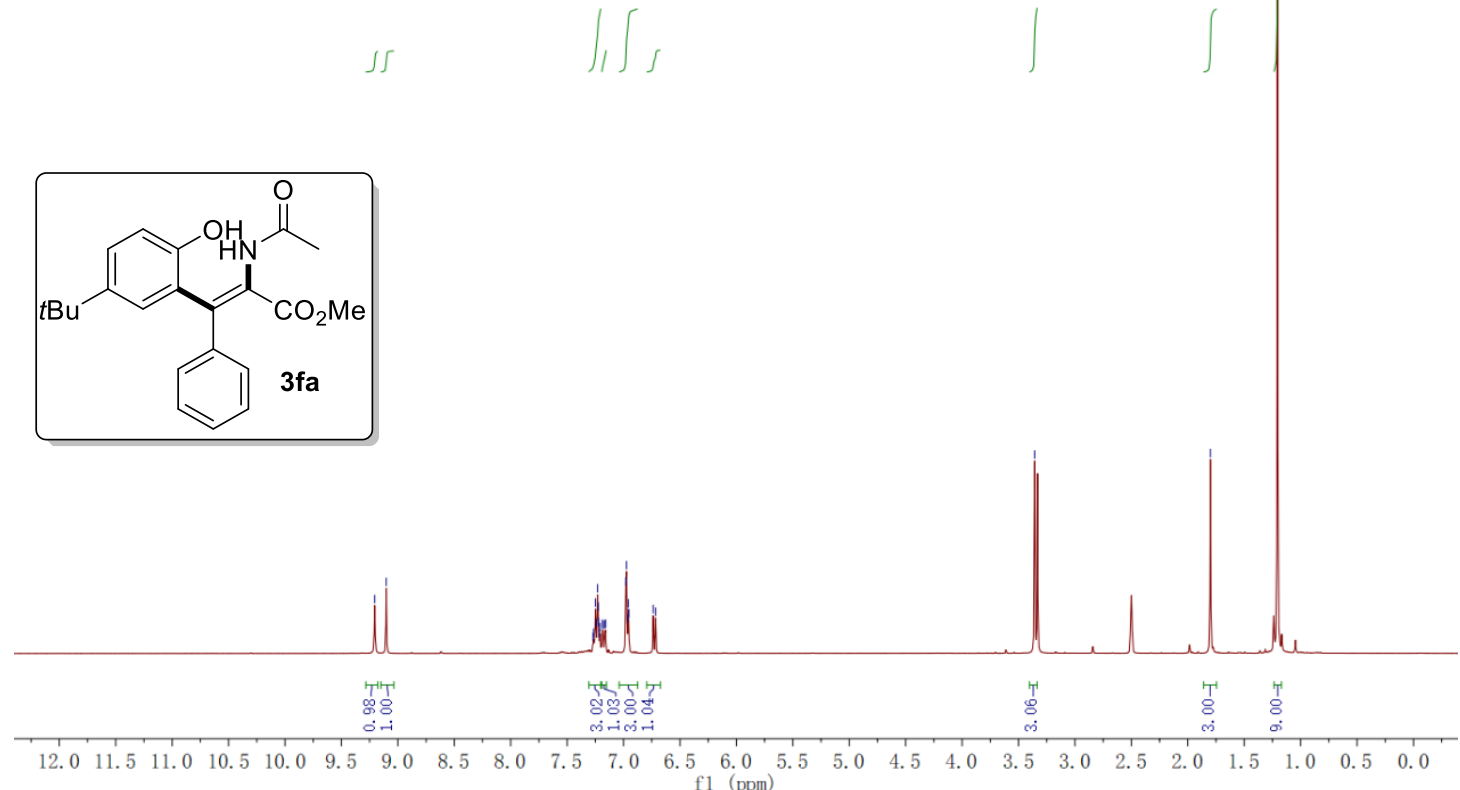

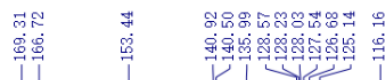
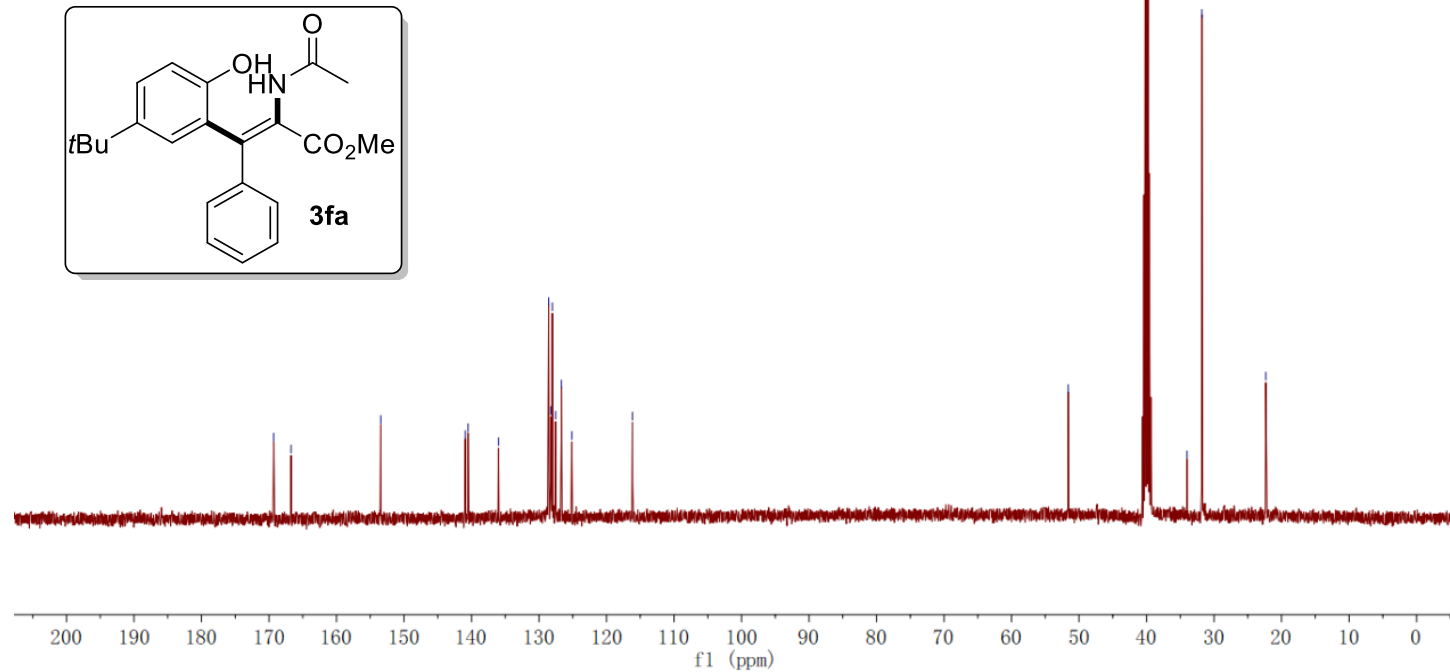
${ }^{1} \mathrm{H}{ }^{13} \mathrm{C}$ and ${ }^{19} \mathrm{~F}$ NMR of 3ga

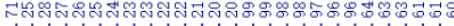

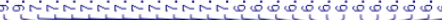
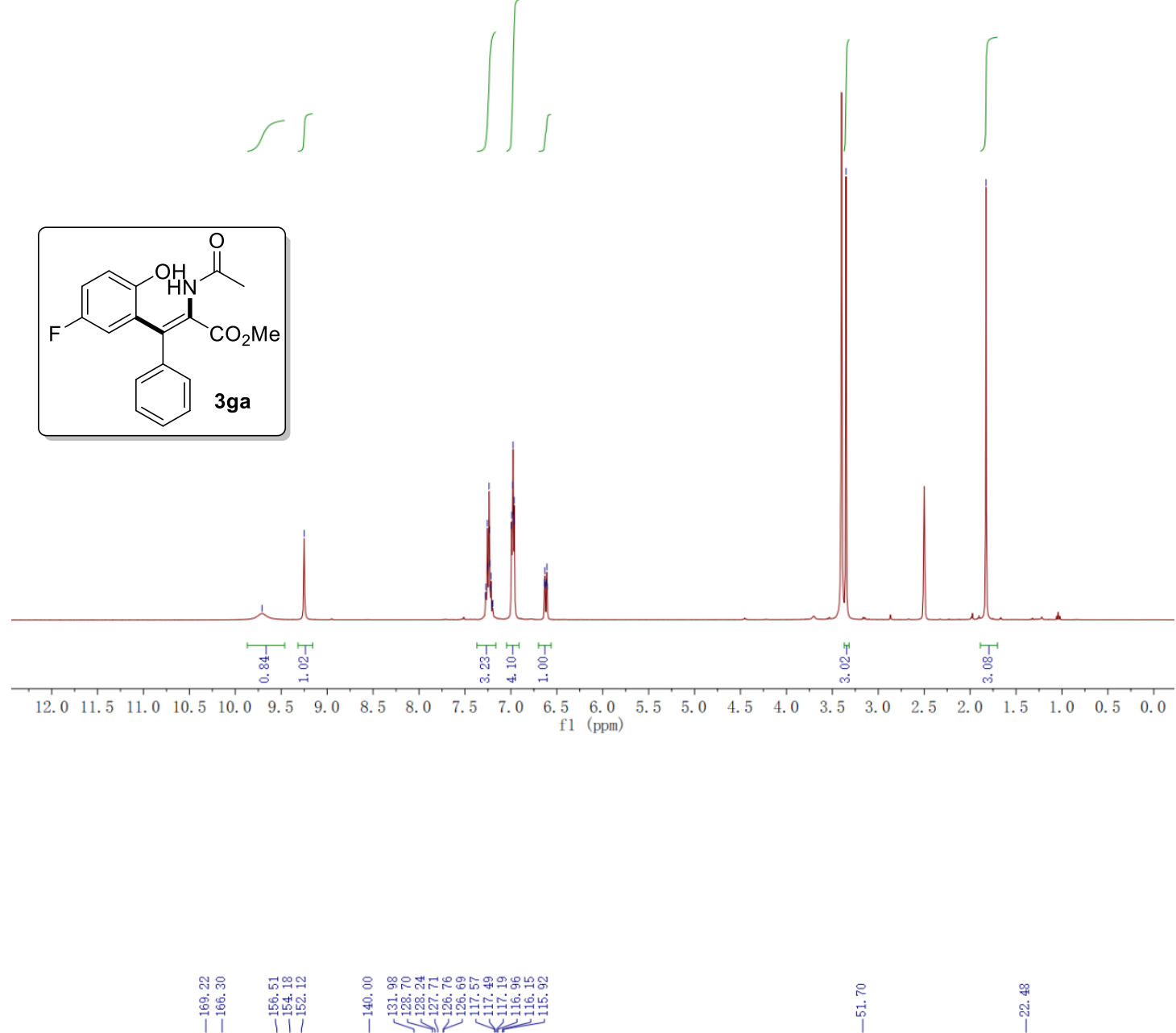

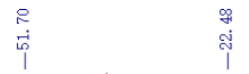
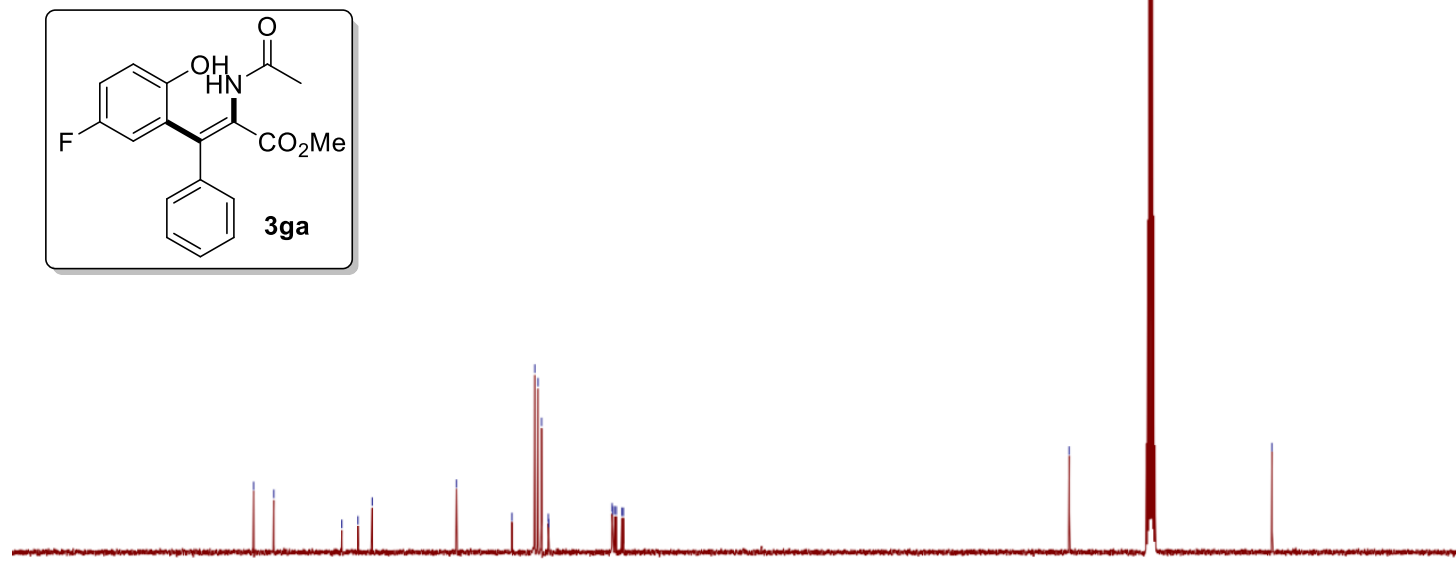

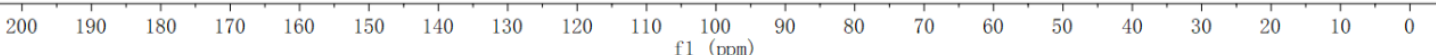




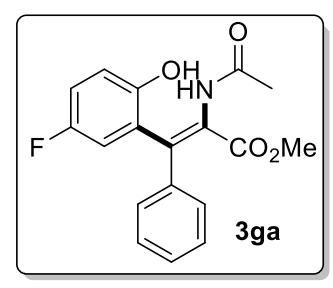

ind

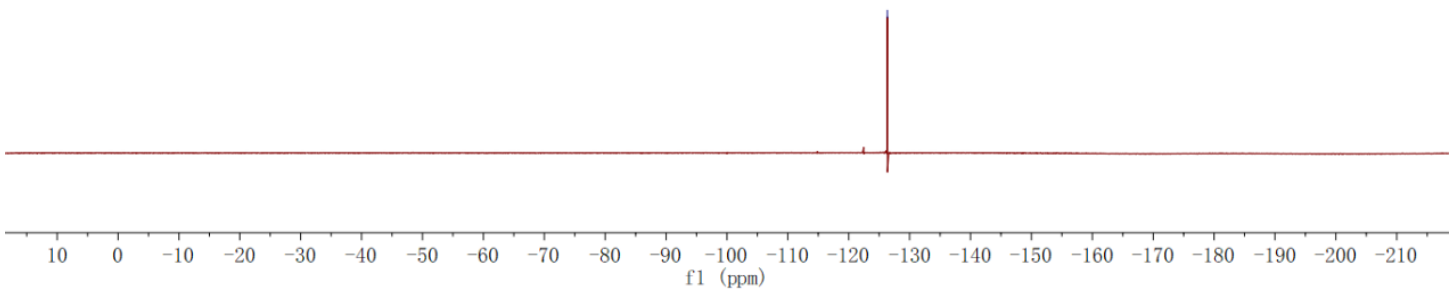

62 
${ }^{1} \mathrm{H}$ and ${ }^{13} \mathrm{C}$ NMR of $\mathbf{3 h a}$

D.
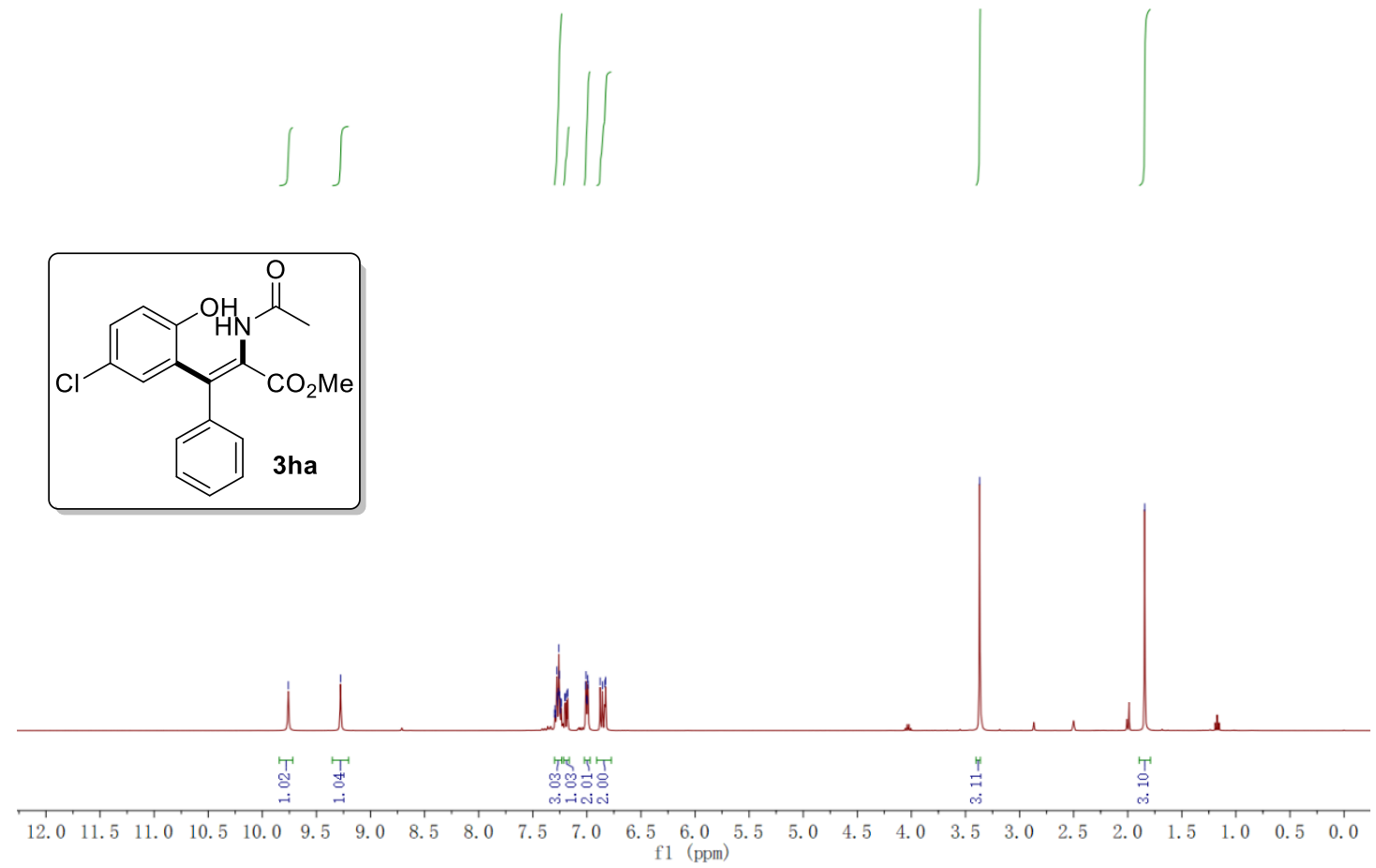

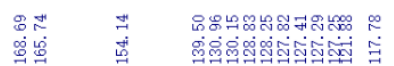

। ।
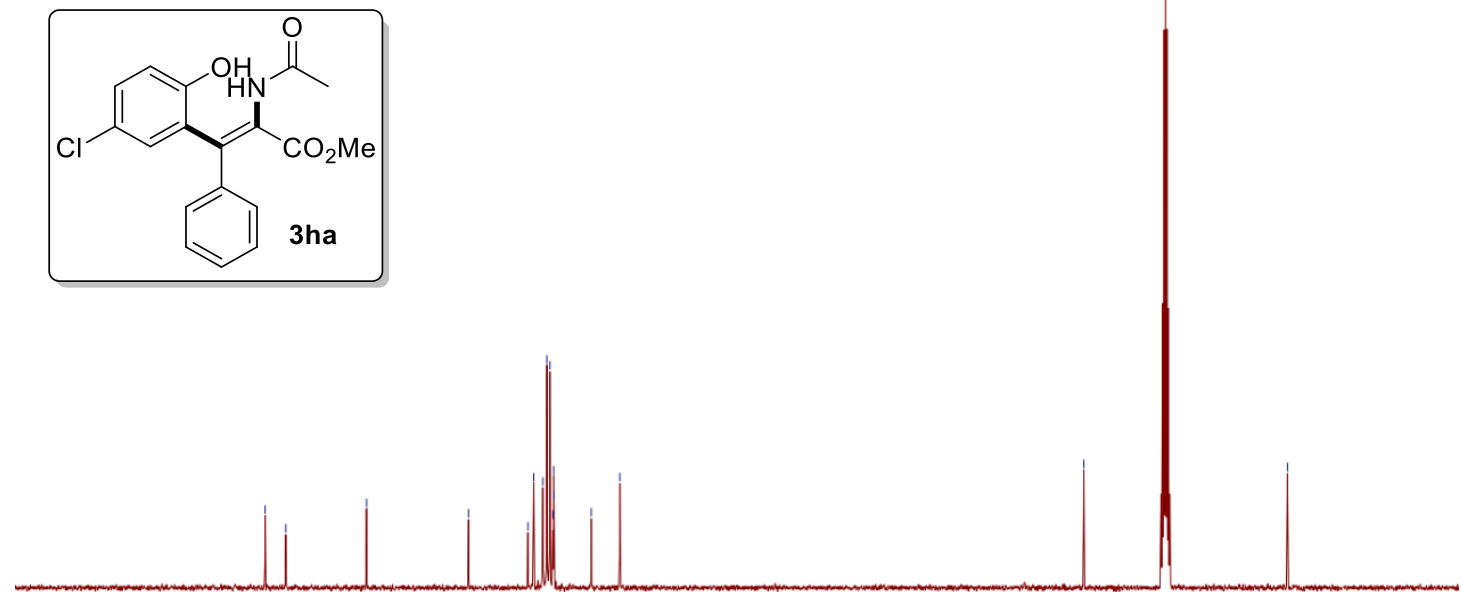

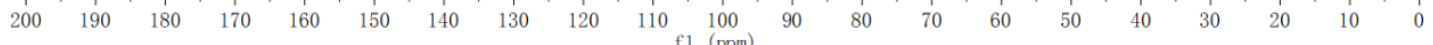


${ }^{1} \mathrm{H}$ and ${ }^{13} \mathrm{C}$ NMR of 3ia

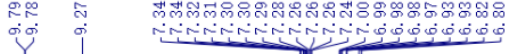

$\stackrel{\infty}{\infty} \stackrel{\infty}{\infty} \underset{i}{\stackrel{\infty}{i}}$
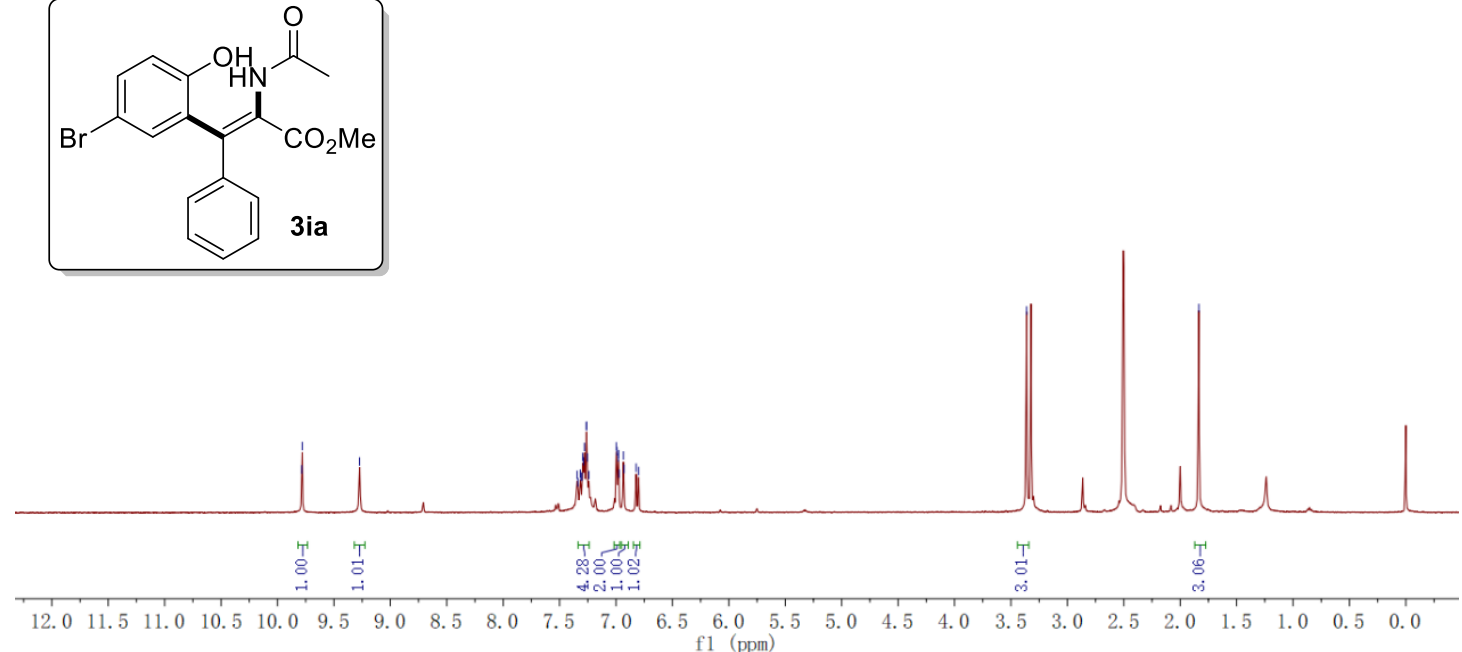

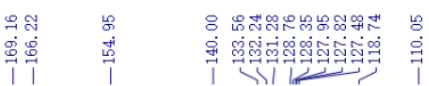

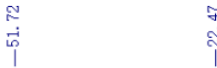
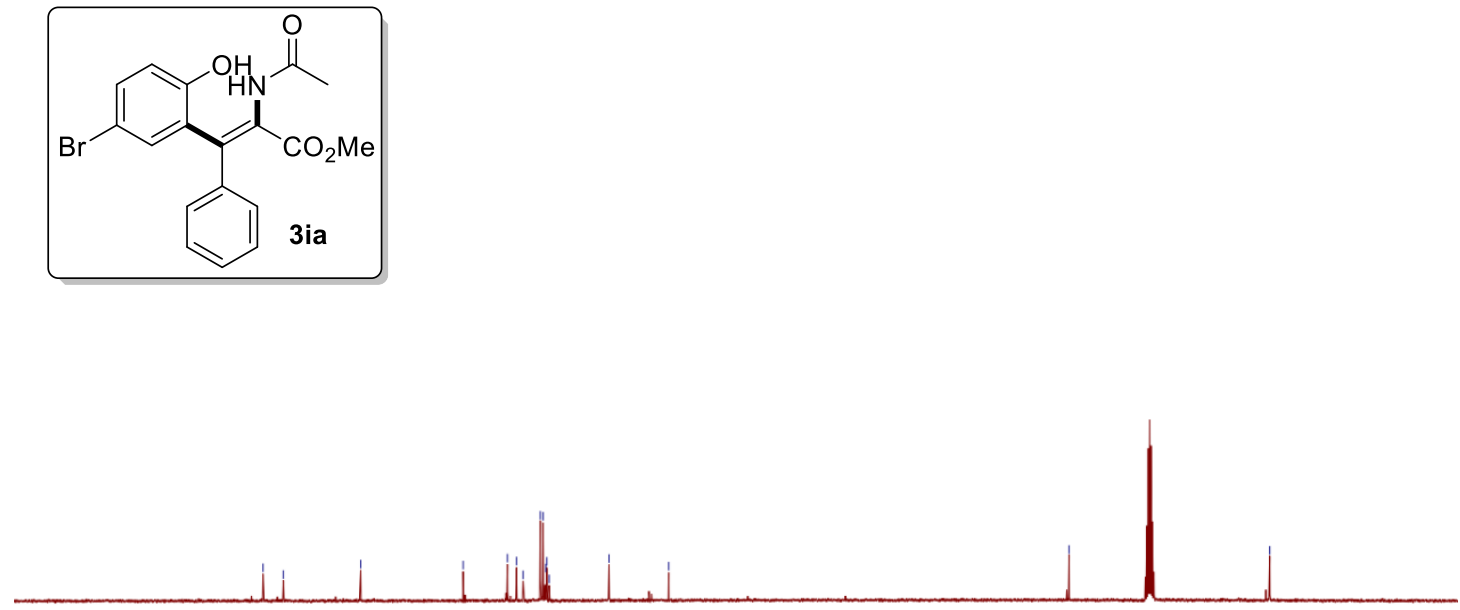

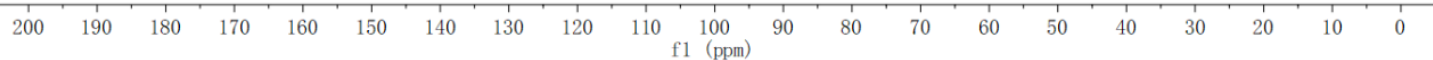


${ }^{1} \mathrm{H}$ and ${ }^{13} \mathrm{C}$ NMR of $\mathbf{3 j a}$

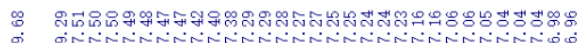
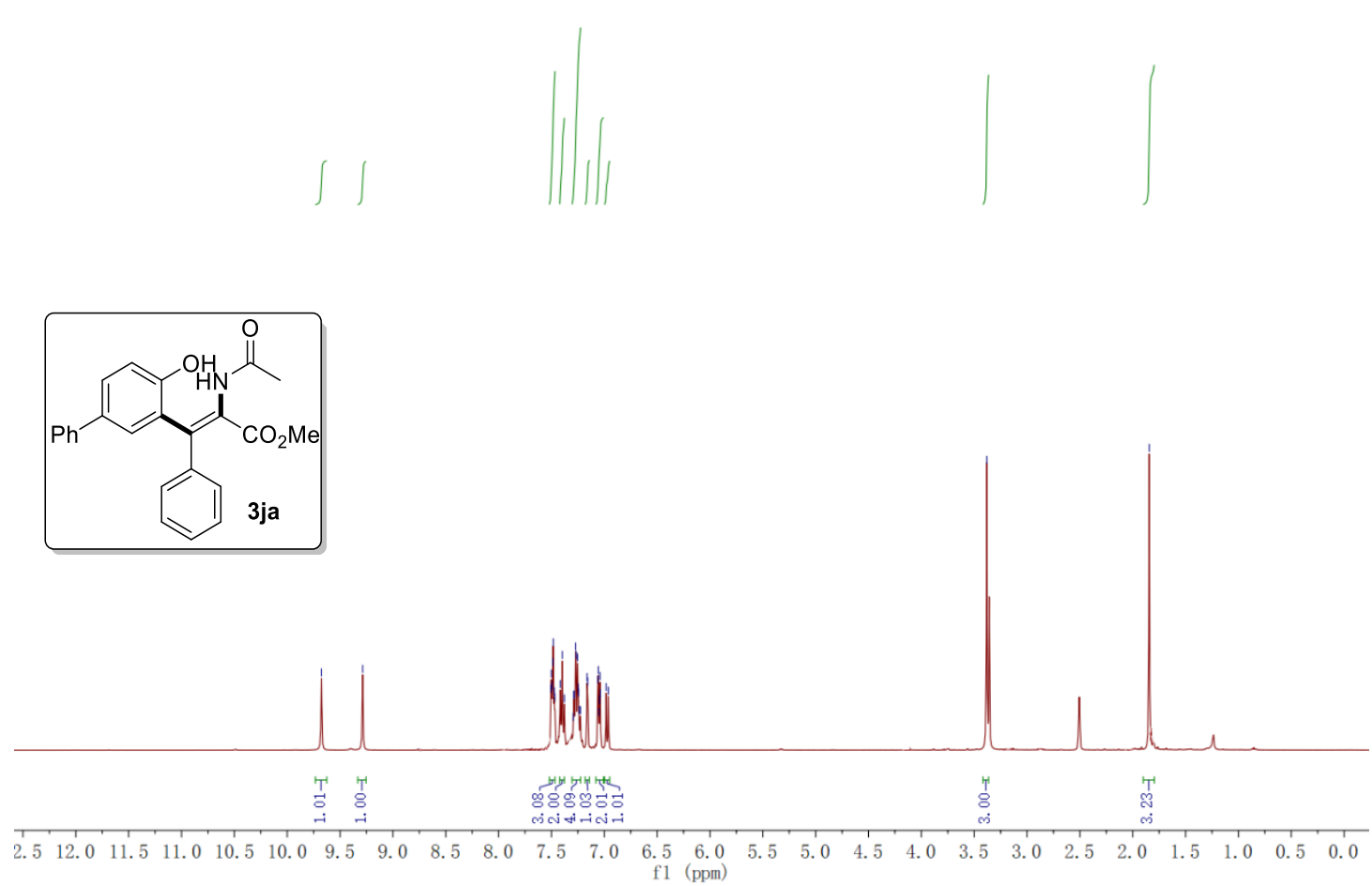

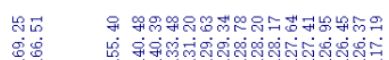

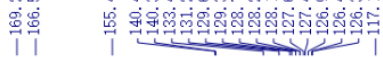
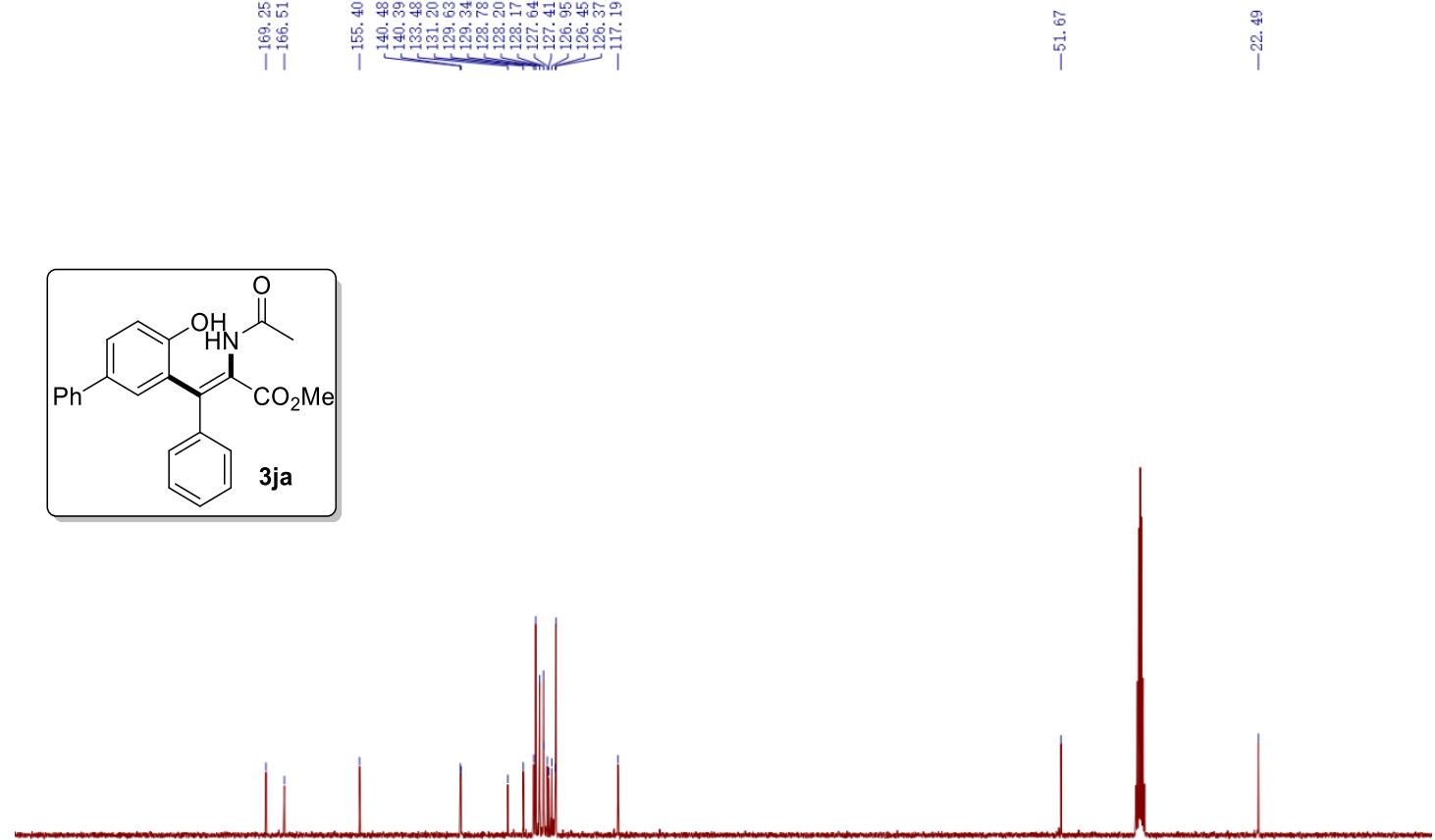

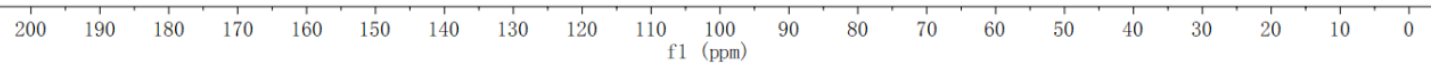


${ }^{1} \mathrm{H}$ and ${ }^{13} \mathrm{C}$ NMR of $\mathbf{3 k a}$

율

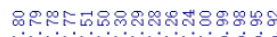

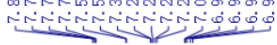

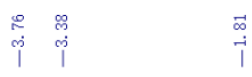

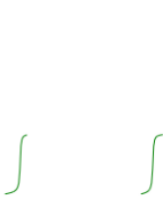

$\iint$
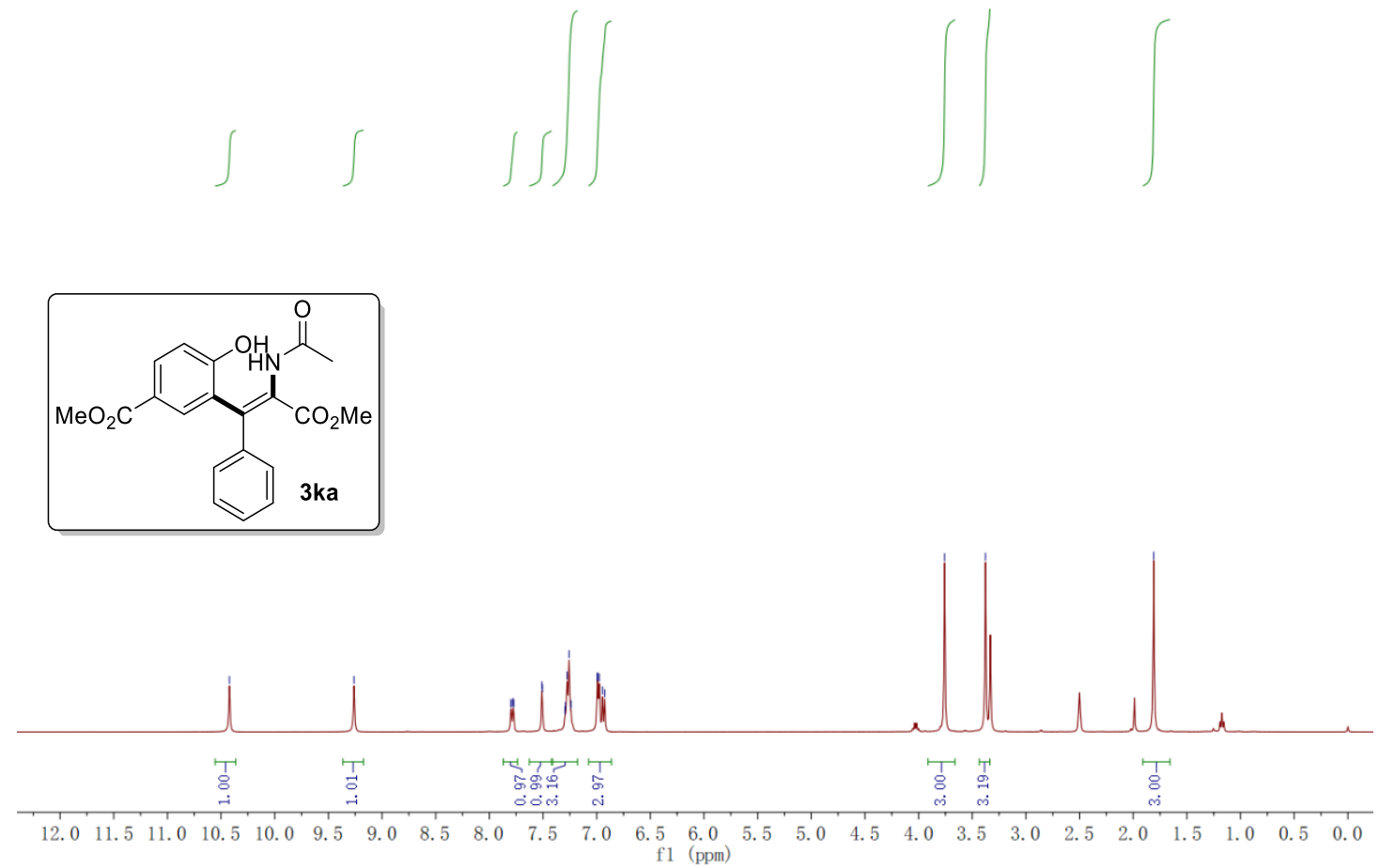

ชุะ

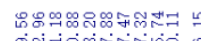

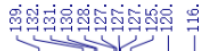

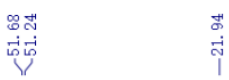
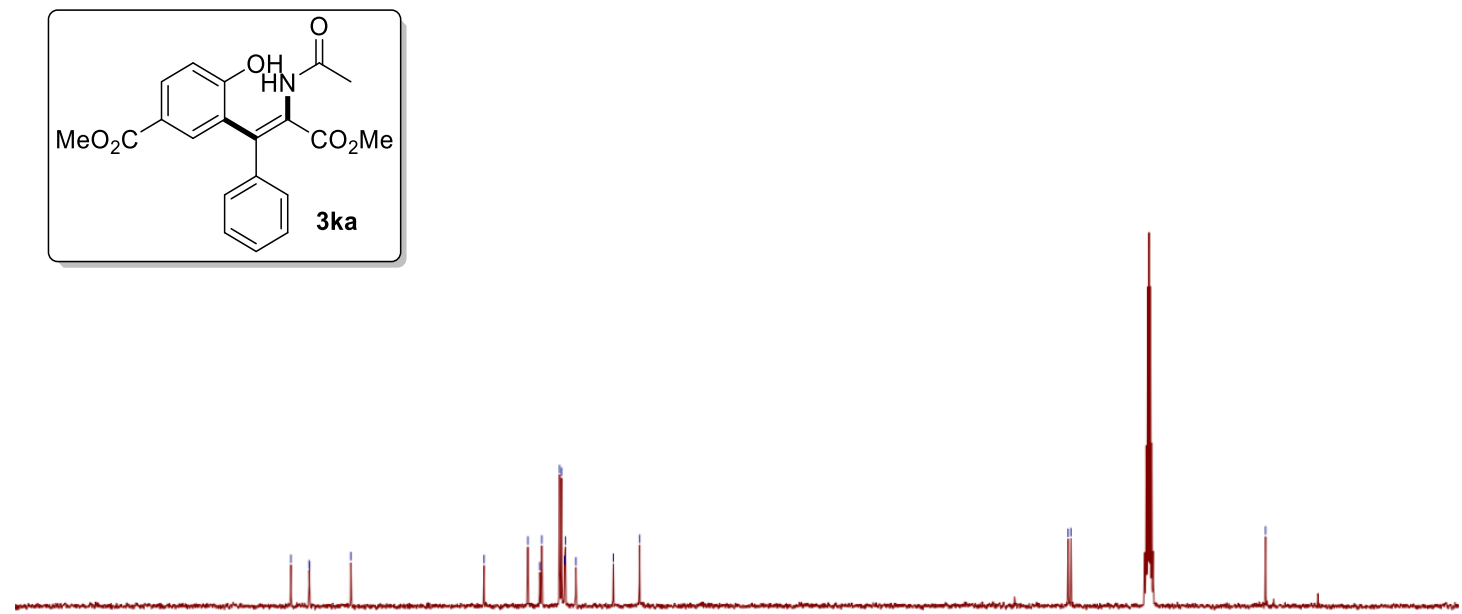

\begin{tabular}{llllllllllllllllllllllllll}
\hline 10 & 200 & 190 & 180 & 170 & 160 & 150 & 140 & 130 & 120 & 110 & 100 & 90 & 80 & 70 & 60 & 50 & 40 & 30 & 20 & 10 & 0
\end{tabular} 
${ }^{1} \mathrm{H}$ and ${ }^{13} \mathrm{C}$ NMR of 3la
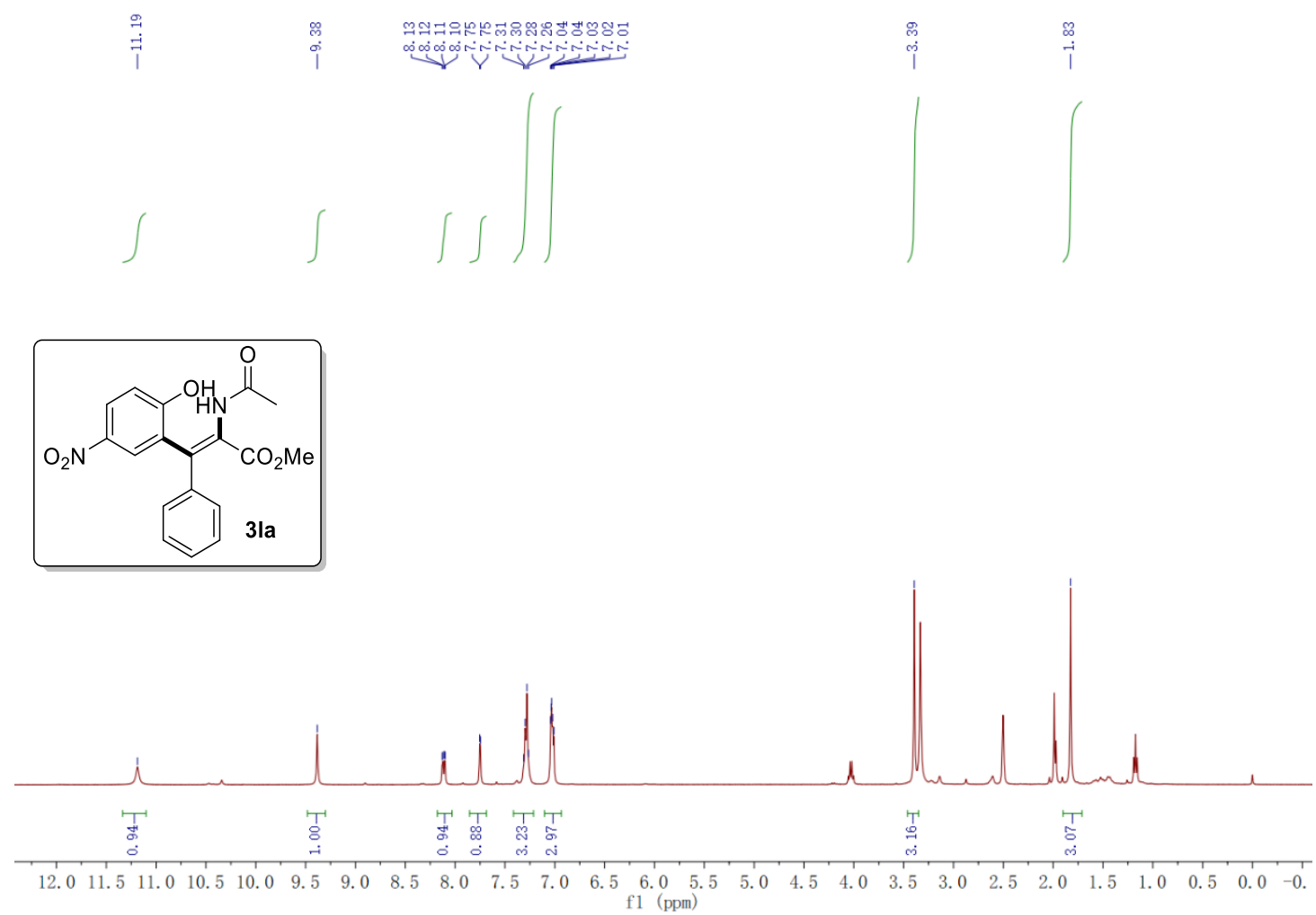

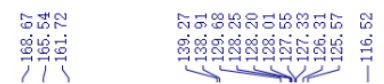
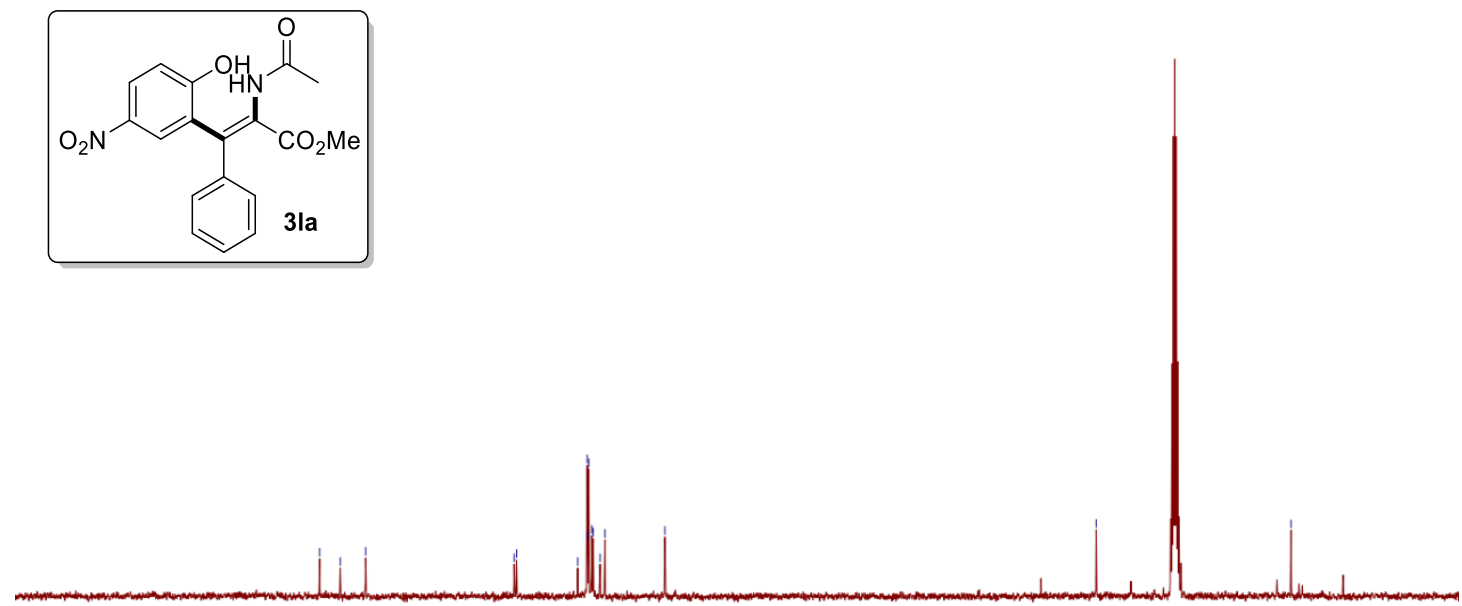

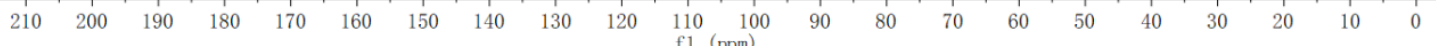


${ }^{1} \mathrm{H}$ and ${ }^{13} \mathrm{C}$ NMR of $\mathbf{3 m a}$

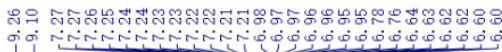

$\underset{\substack{1 \\ 0}}{1}$
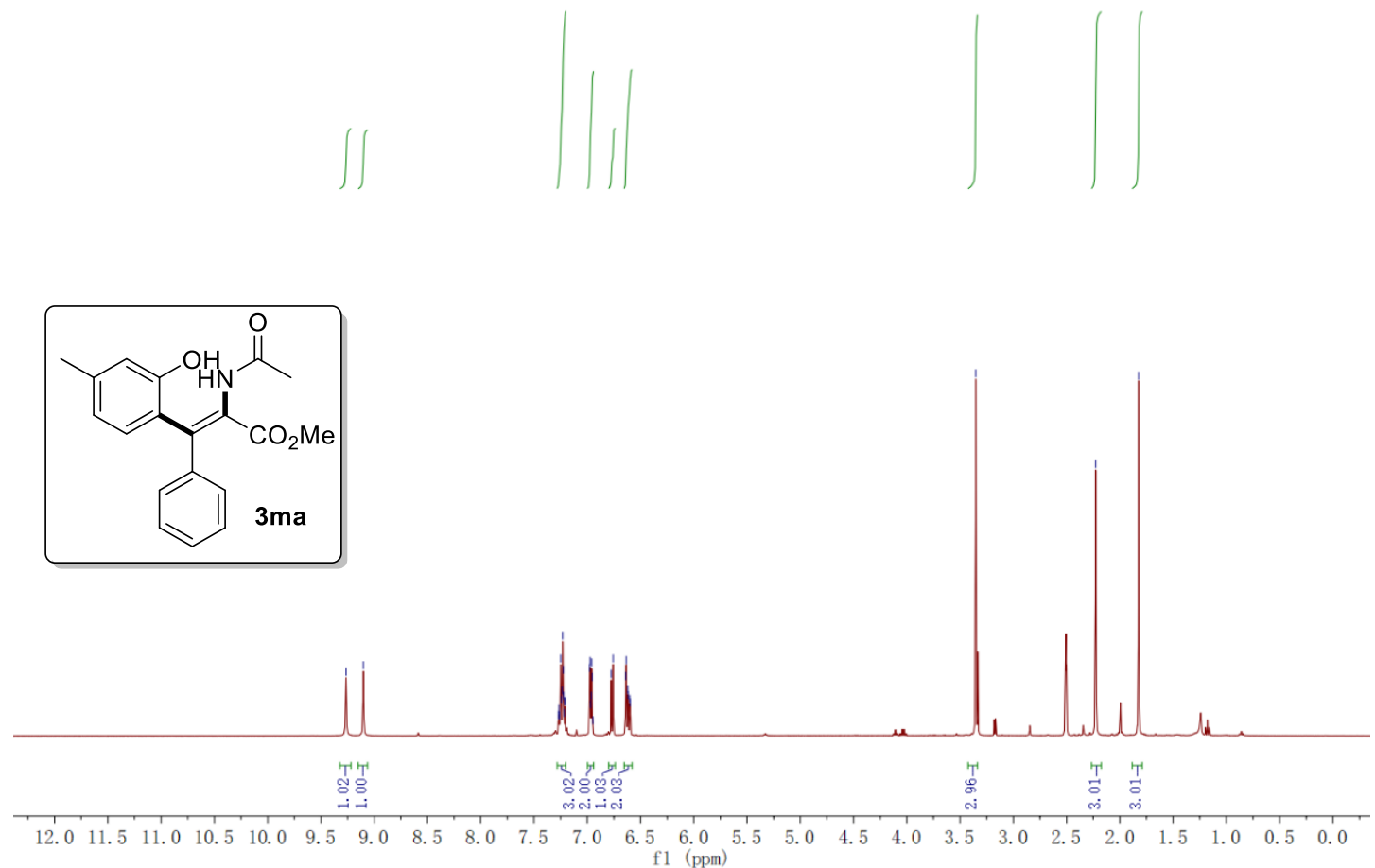

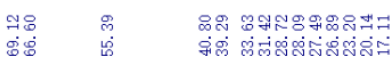

宊

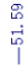

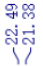
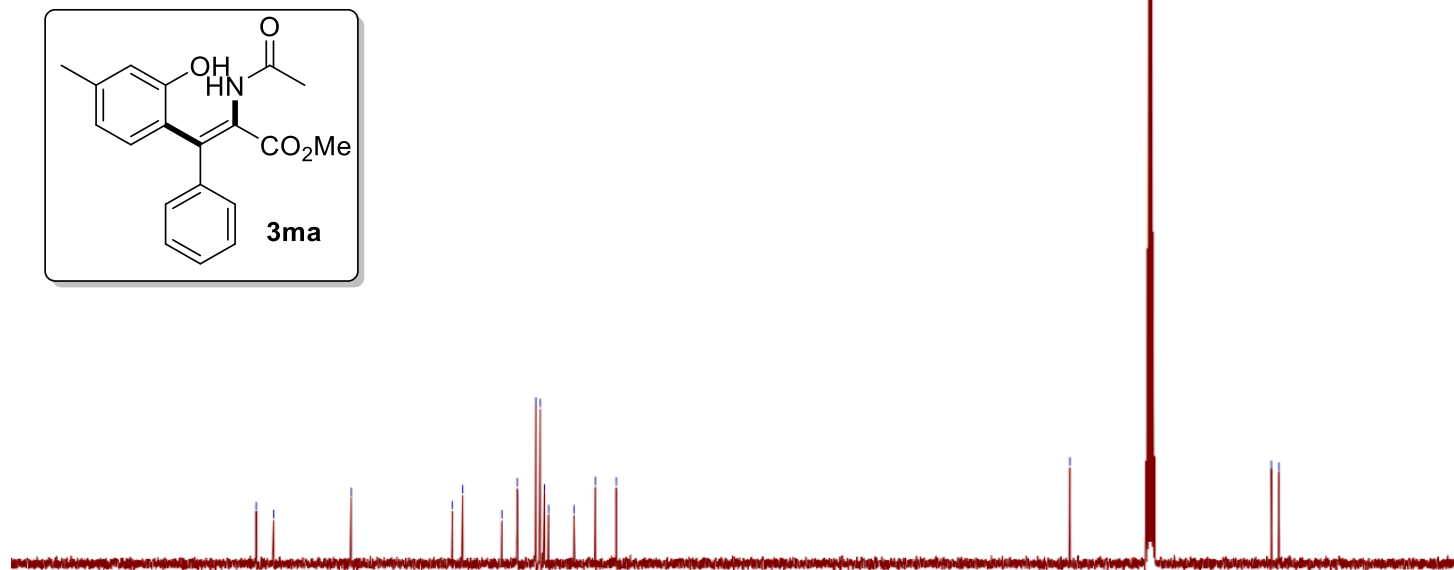

$\begin{array}{lllllllllllllllllllll}200 & 190 & 180 & 170 & 160 & 150 & 140 & 130 & 120 & 110 & 100 & 90 & 80 & 70 & 60 & 50 & 40 & 30 & 20 & 10 & 0\end{array}$ 
${ }^{1} \mathrm{H}{ }^{13} \mathrm{C}$ and ${ }^{19} \mathrm{~F}$ NMR of 3 na

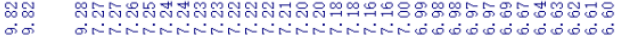
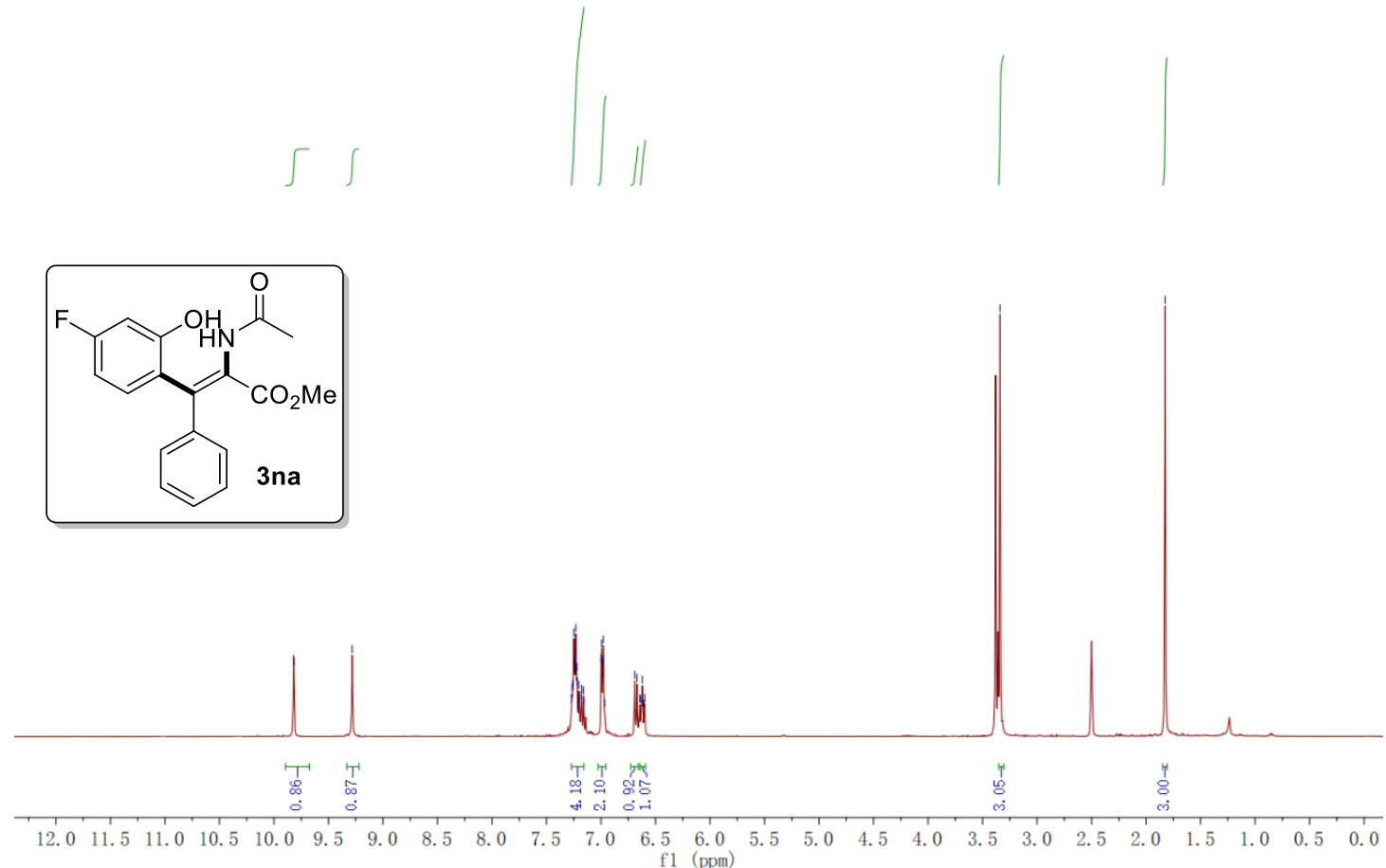

\section{ร mก}

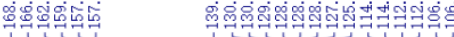

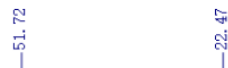

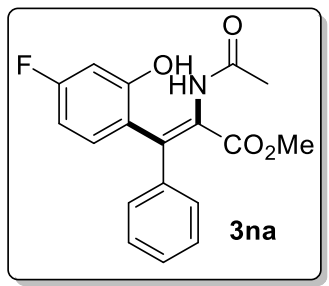

$\begin{array}{lllllllllll}200 & 190 & 180 & 170 & 160 & 150 & 140 & 130 & 120 & 110 & 100\end{array}$

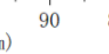

70

$60 \quad 50$

40

$20 \quad 10 \quad 0$ 

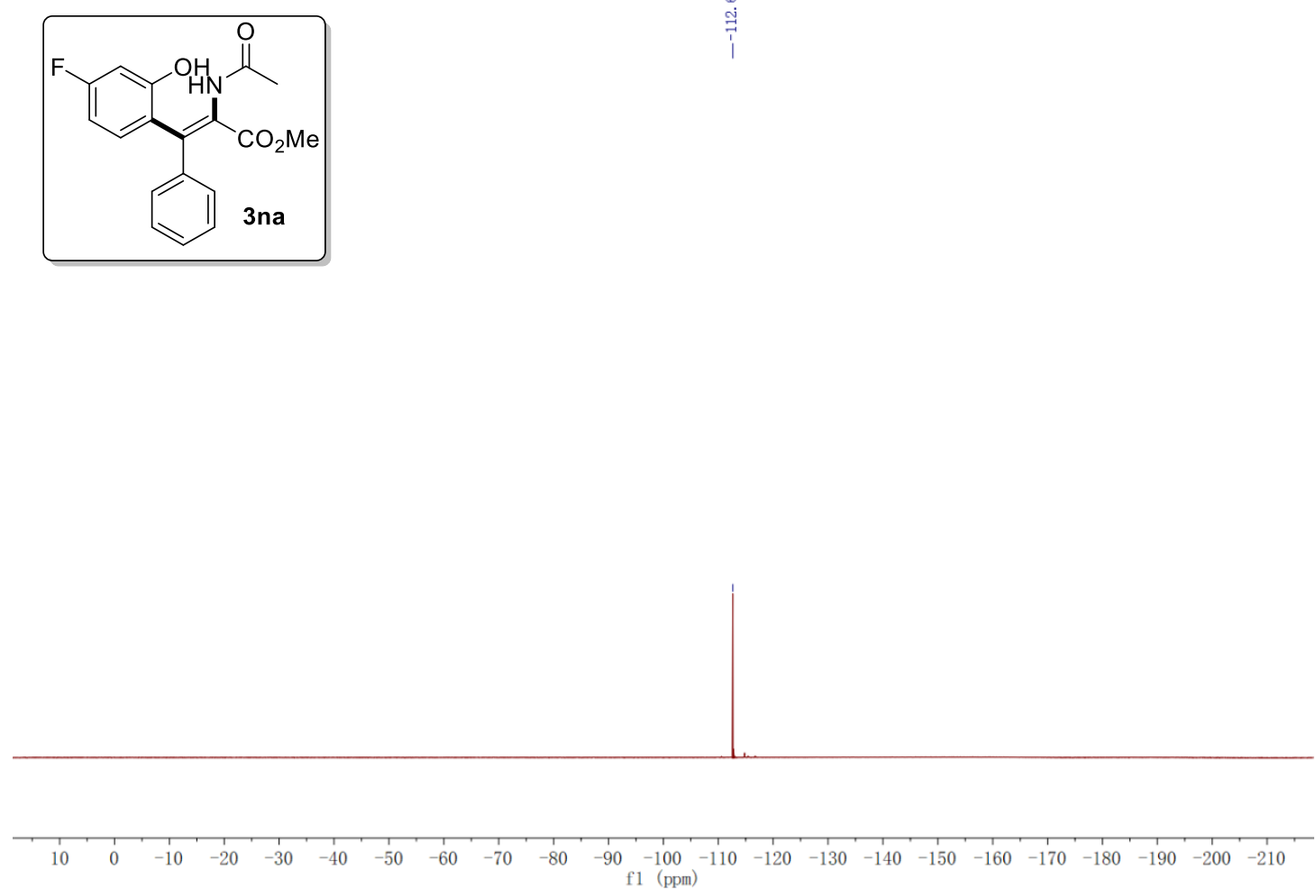
${ }^{1} \mathrm{H}$ and ${ }^{13} \mathrm{C}$ NMR of $30 a$
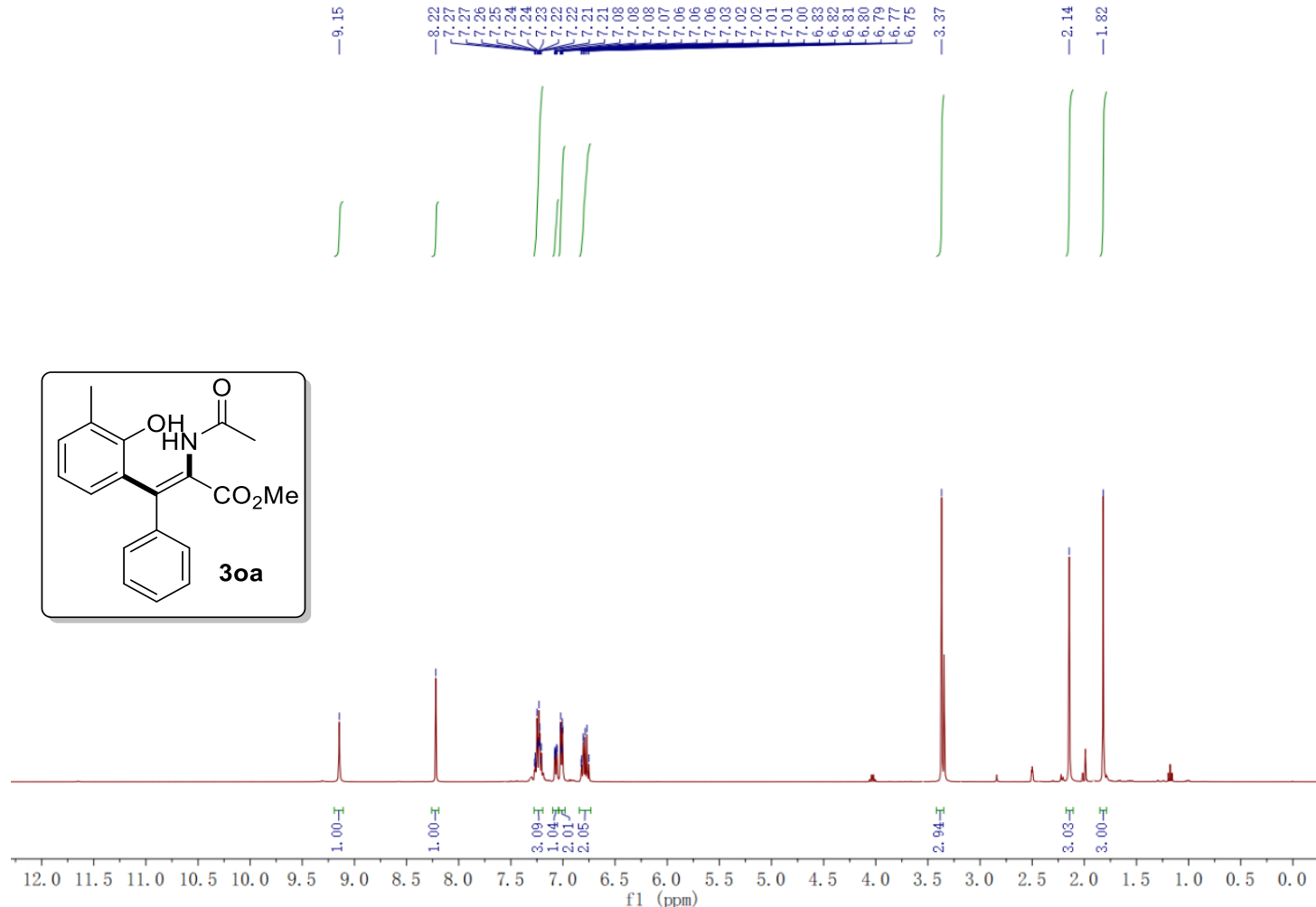

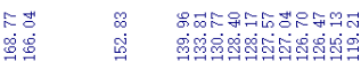

। ।

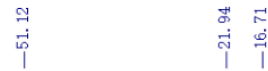
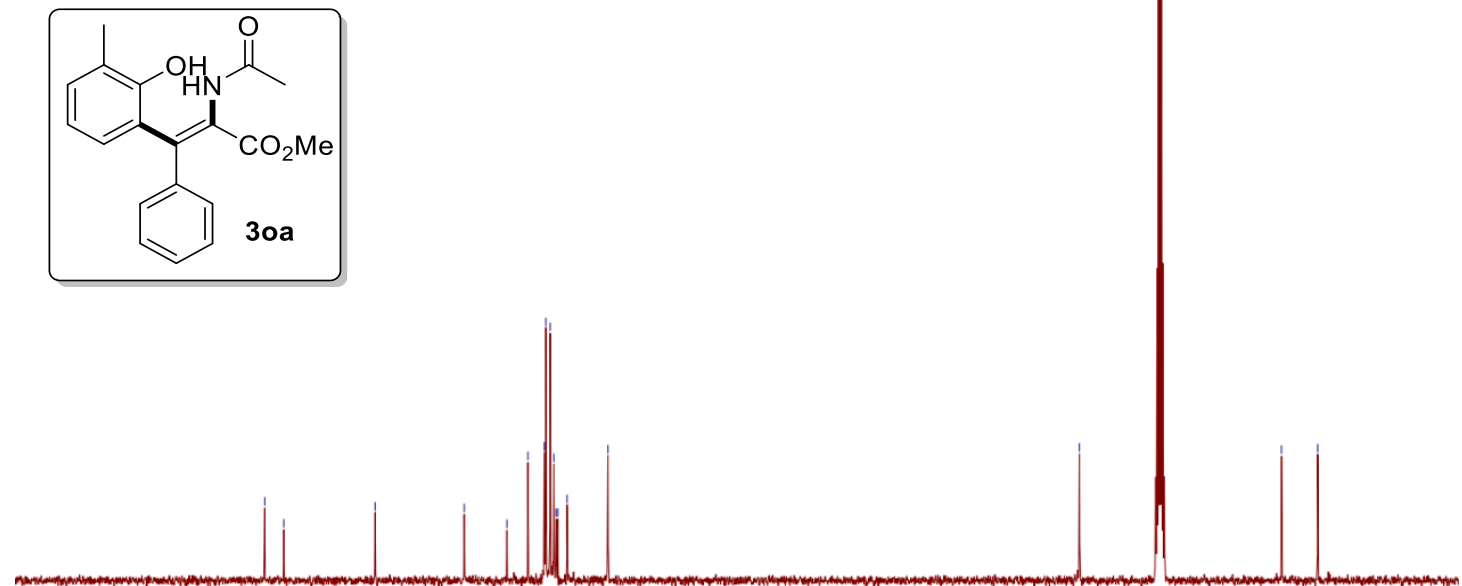

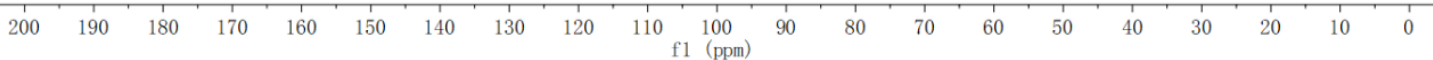


${ }^{1} \mathrm{H}$ and ${ }^{13} \mathrm{C}$ NMR of $\mathbf{3 p a}$
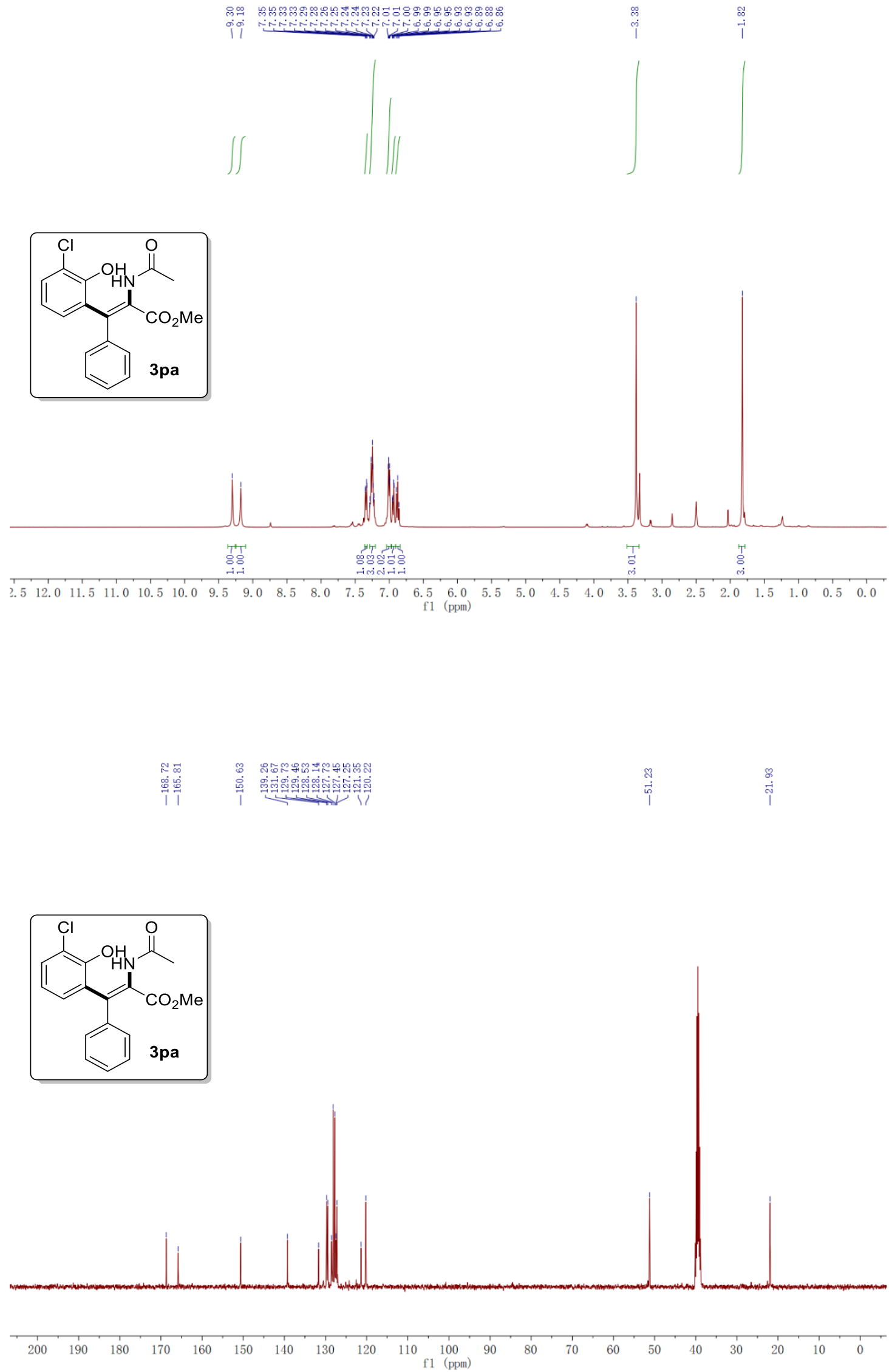
${ }^{1} \mathrm{H}$ and ${ }^{13} \mathrm{C}$ NMR of $\mathbf{3 q a}$
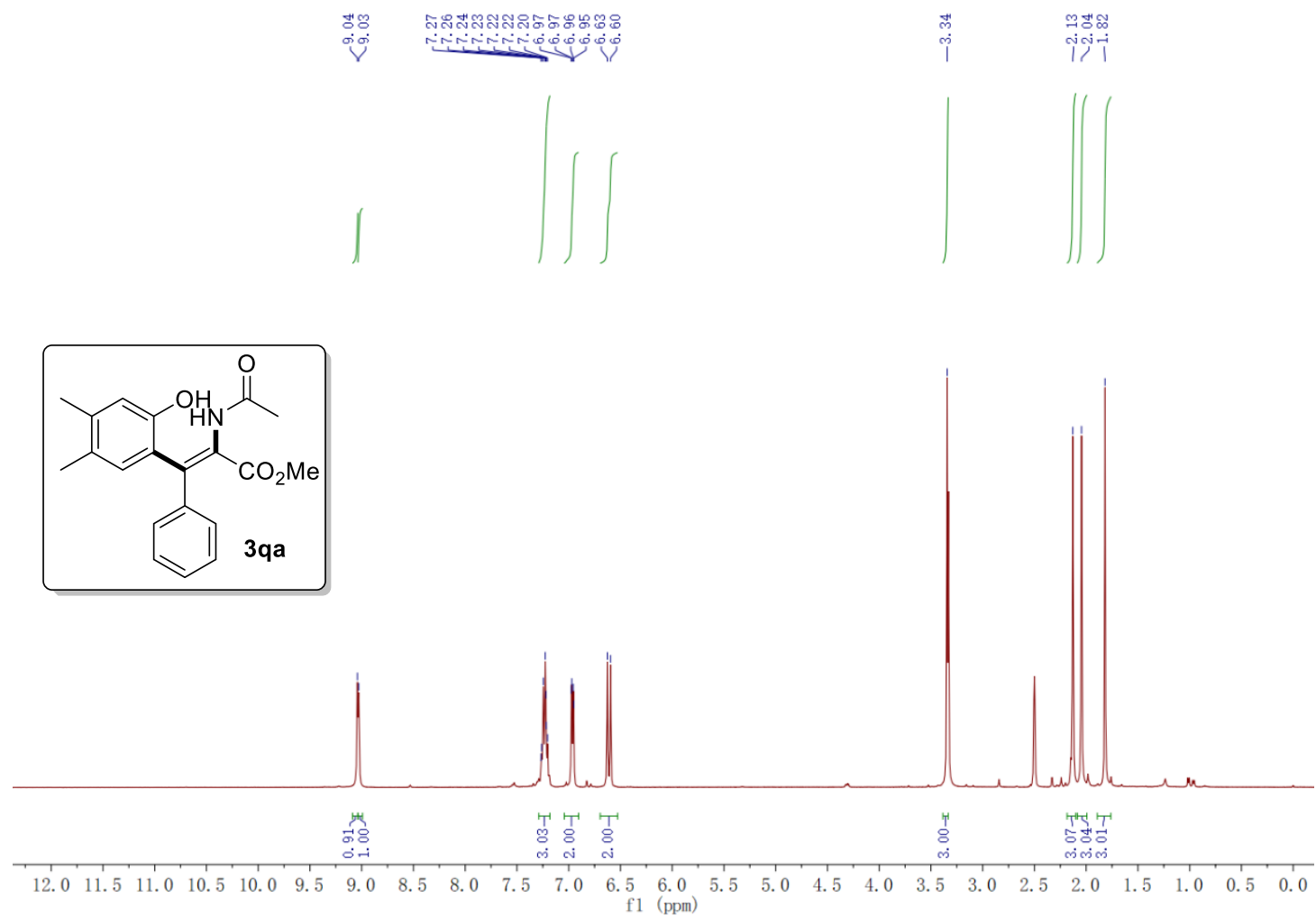

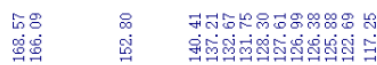
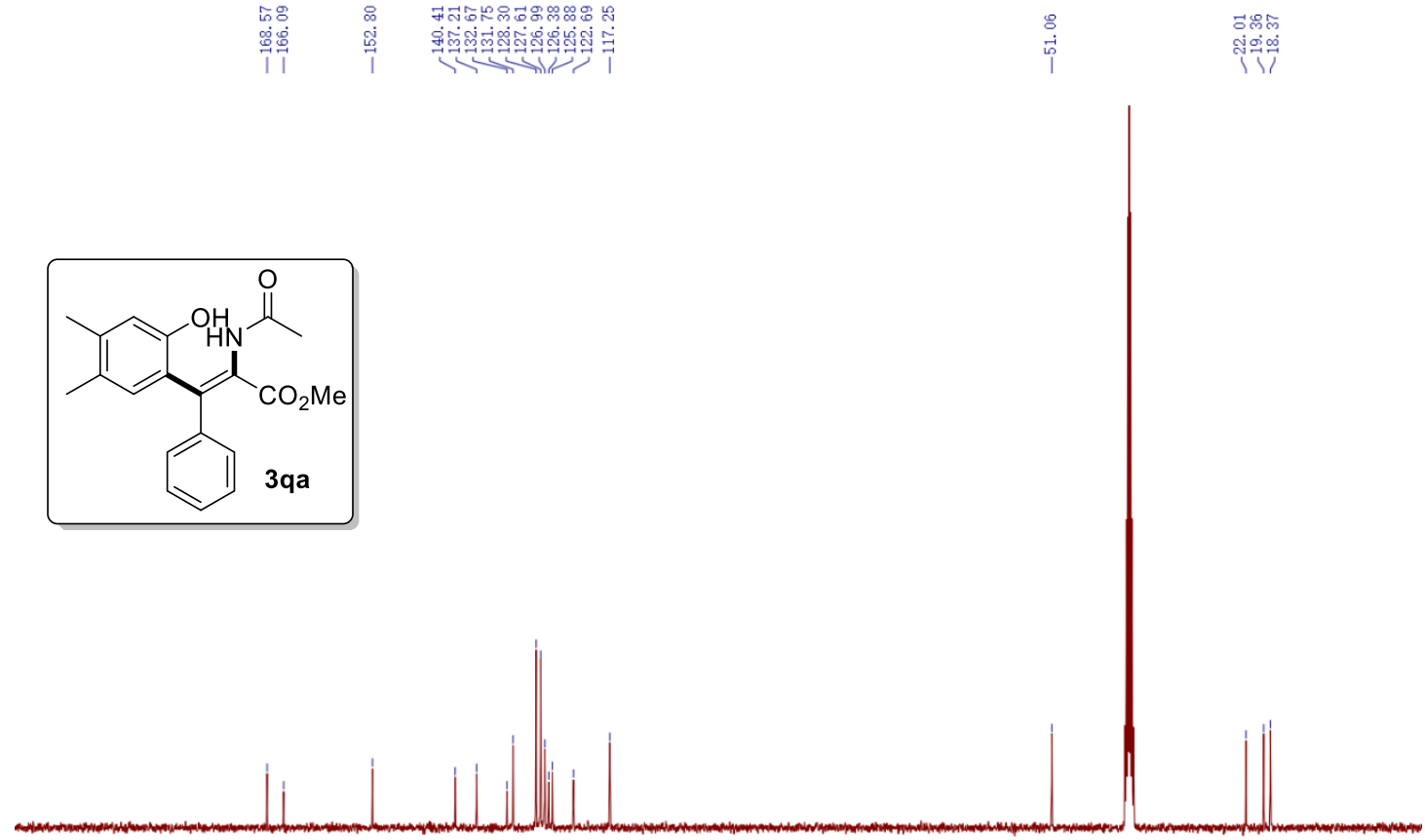

200

$\begin{array}{lllllllll}180 & 170 & 160 & 150 & 140 & 130 & 120 & 110 & 100\end{array}$

90

70

(n)

$\begin{array}{rrrr}10 & 10 & 1 & 1 \\ 30 & 20 & 10 & 0\end{array}$ 
${ }^{1} \mathrm{H}$ and ${ }^{13} \mathrm{C}$ NMR of 3ra
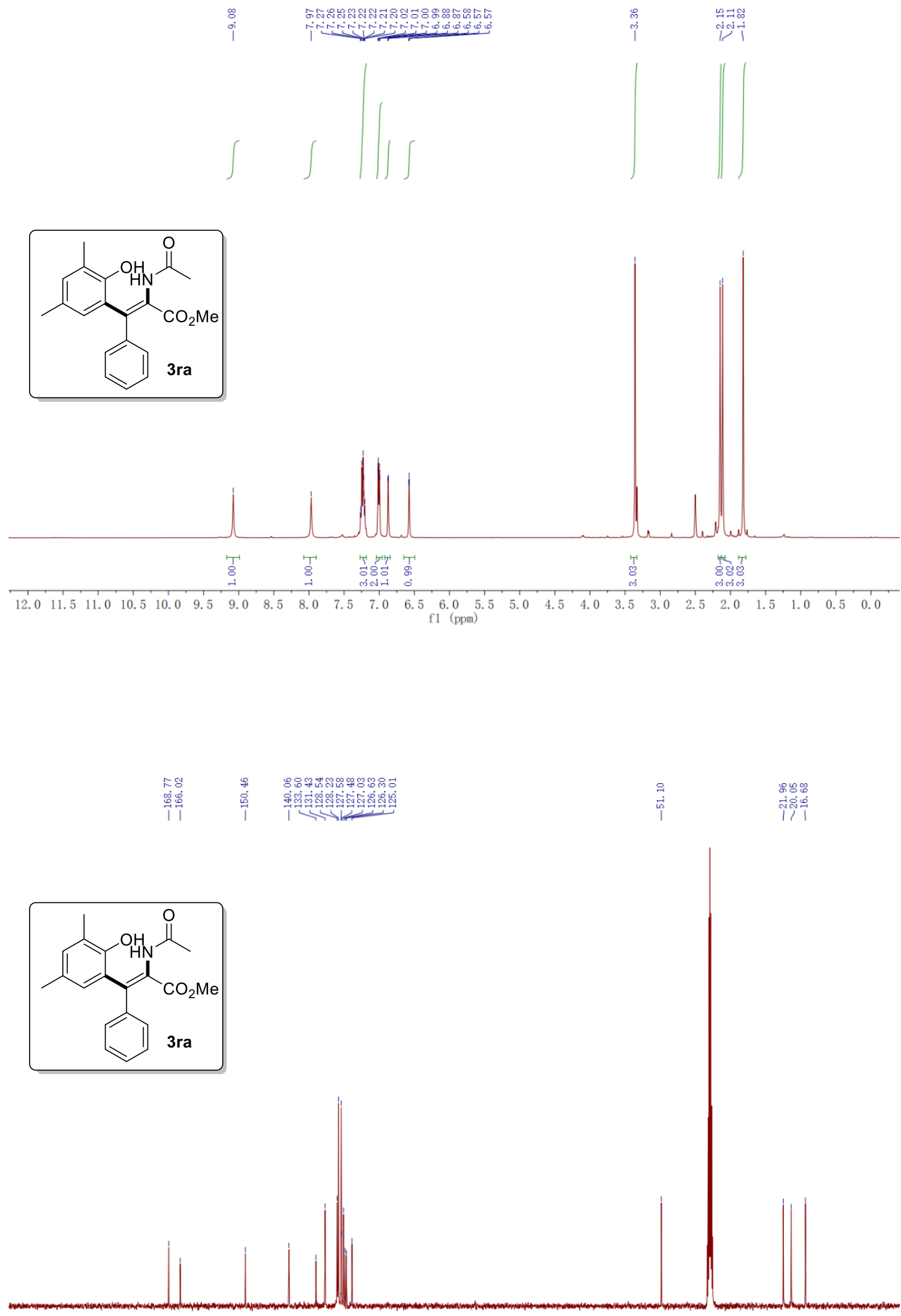

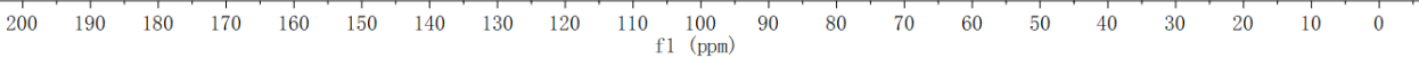


${ }^{1} \mathrm{H}$ and ${ }^{13} \mathrm{C}$ NMR of 3sa
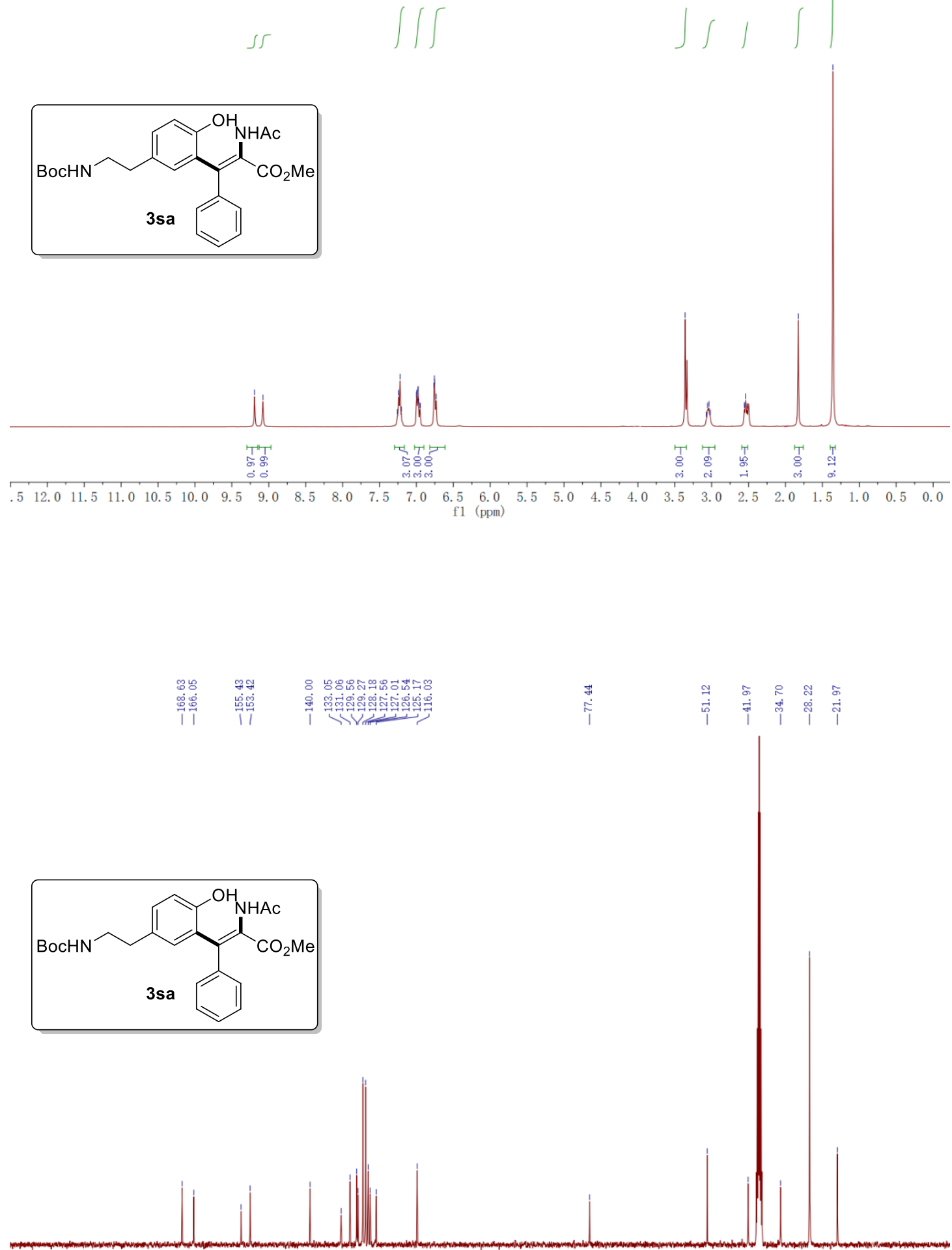

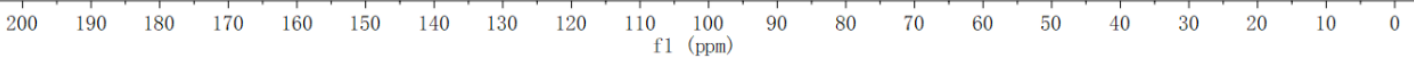


${ }^{1} \mathrm{H}$ and ${ }^{13} \mathrm{C}$ NMR of 3 ta

I)

5s

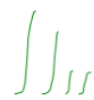

3ta

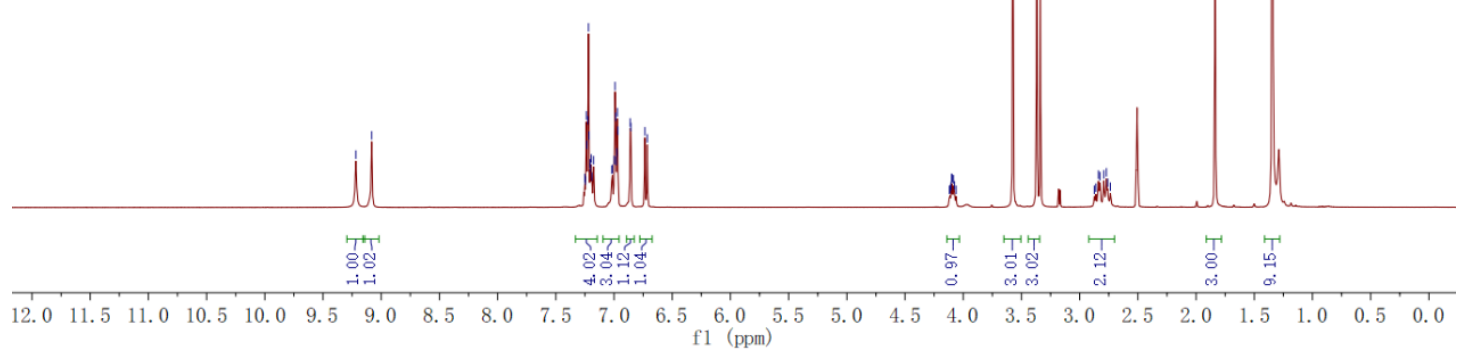

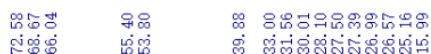

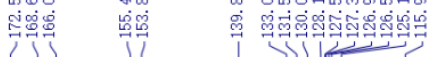

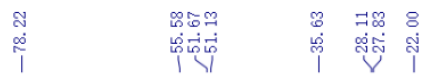

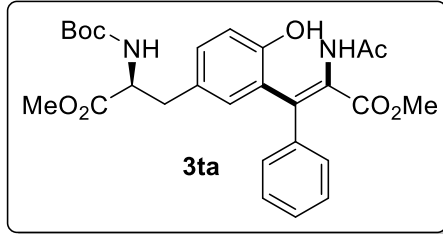

200

$190 \quad 180$

$170-160-150$

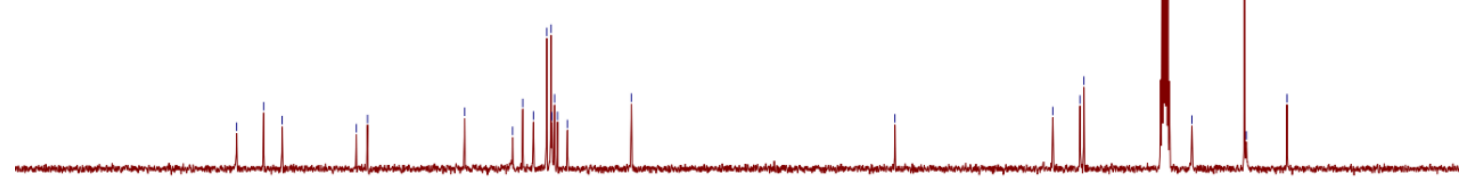


${ }^{1} \mathrm{H}$ and ${ }^{13} \mathrm{C}$ NMR of 3 ua

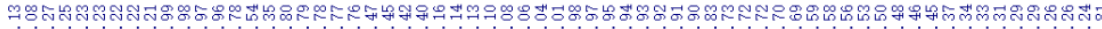

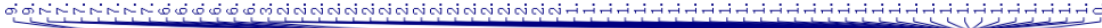

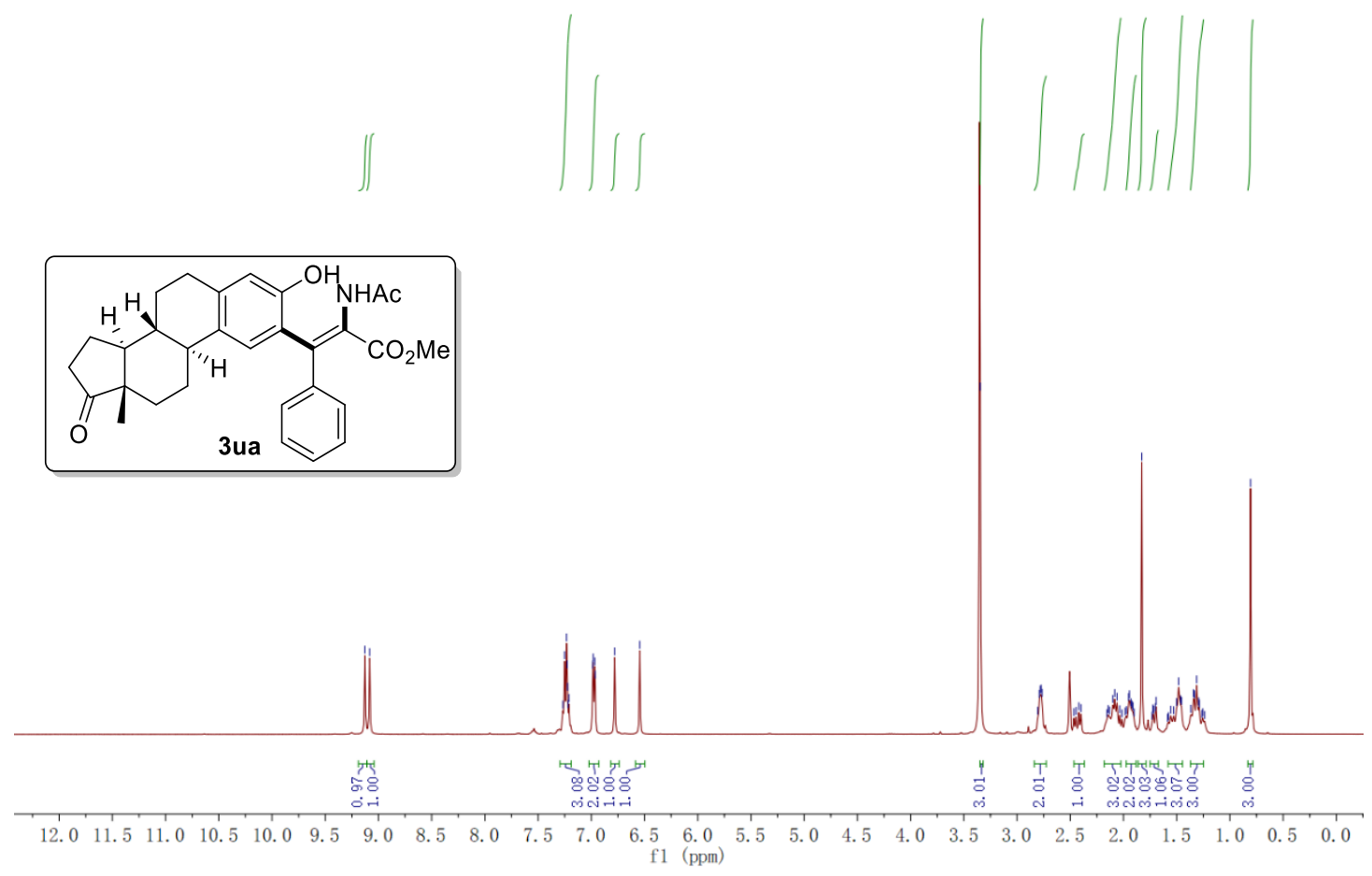

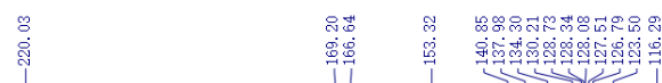

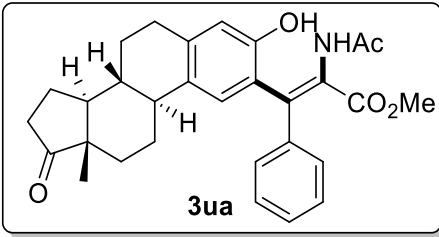

$\begin{array}{lllllllllllllllllllllll}220 & 210 & 200 & 190 & 180 & 170 & 160 & 150 & 140 & 130 & 120 & 110 & 100 & 90 & 80 & 70 & 60 & 50 & 40 & 30 & 20 & 10 & 0\end{array}$ 
${ }^{1} \mathrm{H}$ and ${ }^{13} \mathrm{C}$ NMR of $\mathbf{3 a b}$

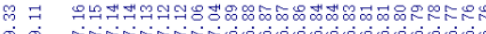

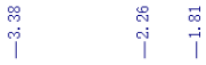

$\iint 1 / 1$
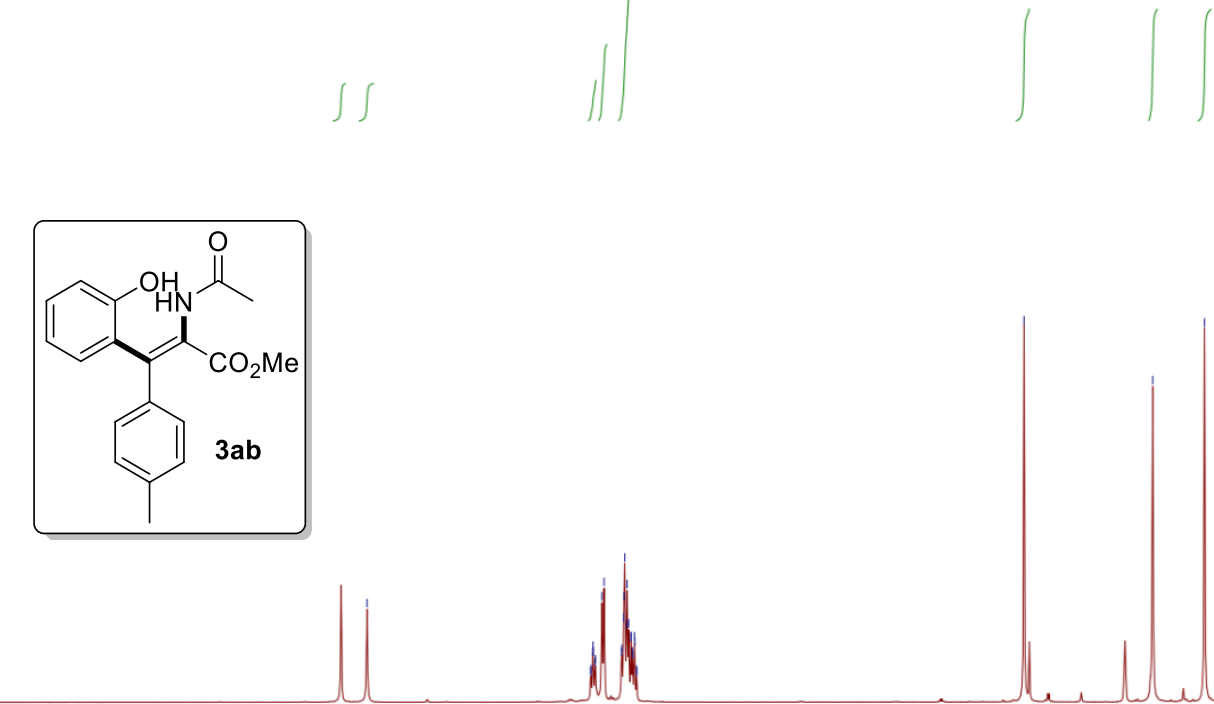

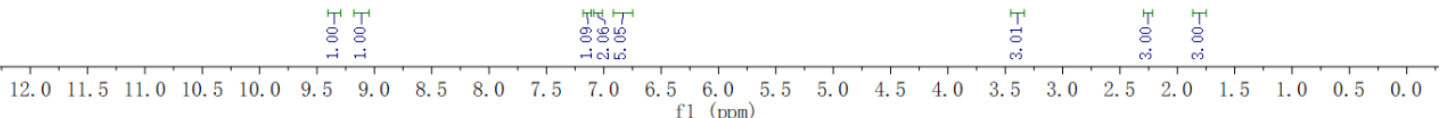

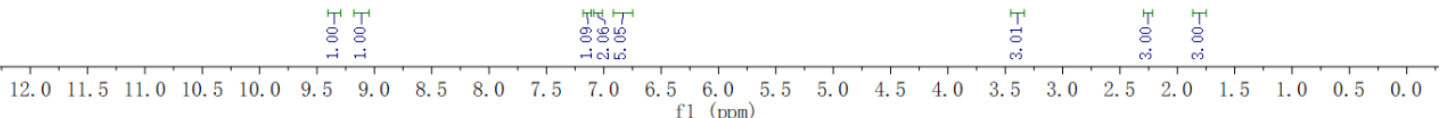

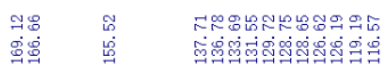

। ।

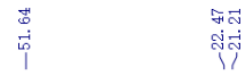
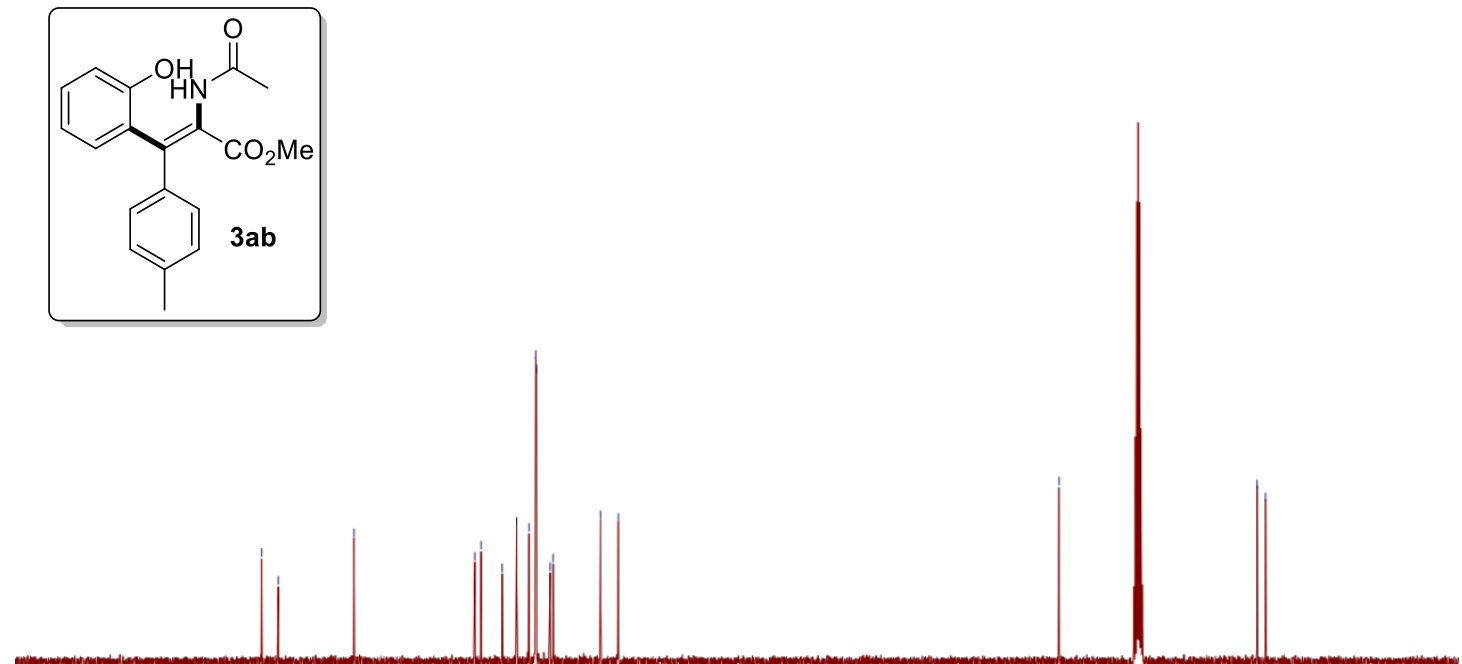

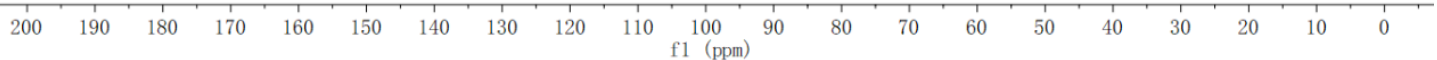


${ }^{1} \mathrm{H}$ and ${ }^{13} \mathrm{C}$ NMR of $\mathbf{3 a c}$
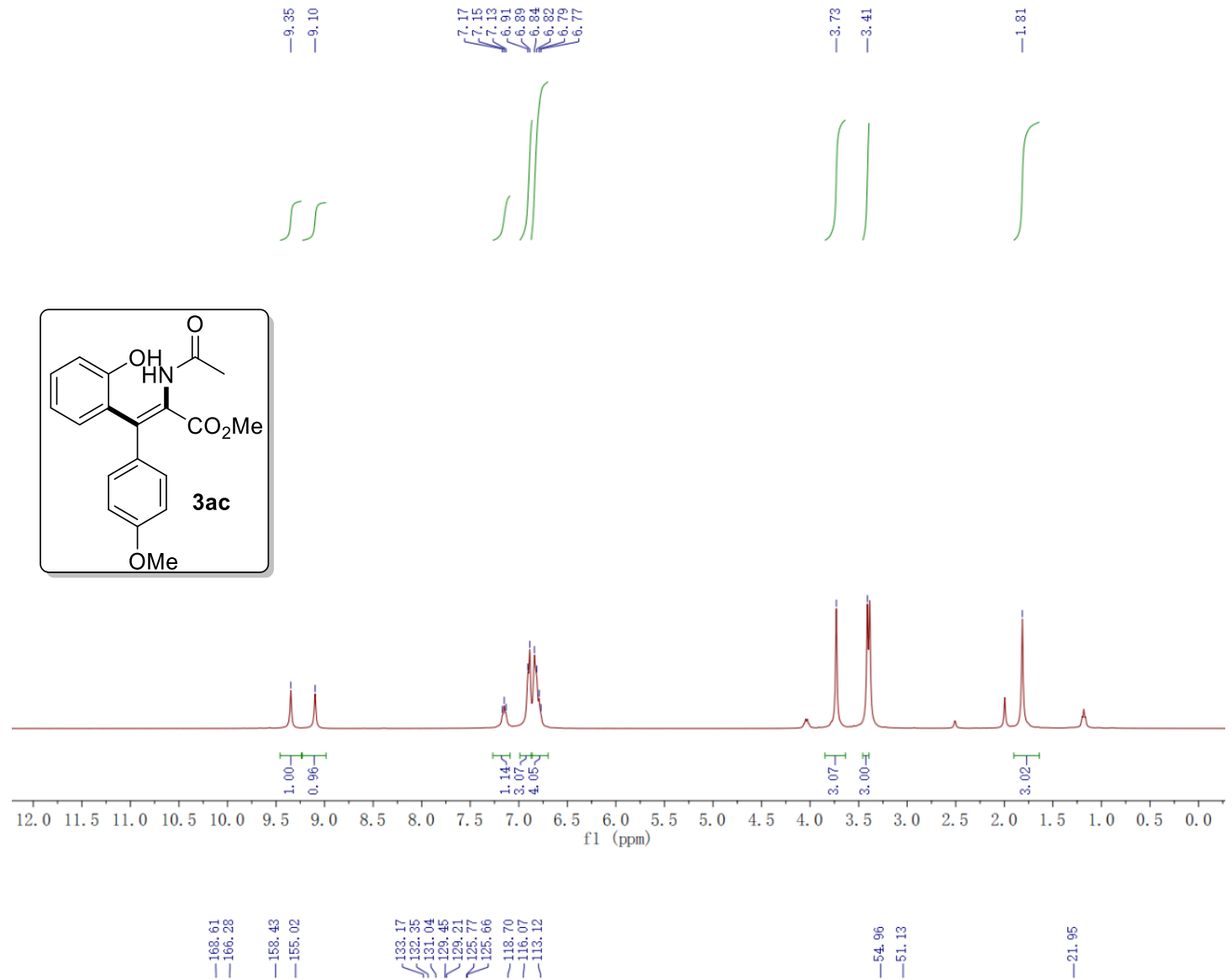

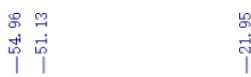
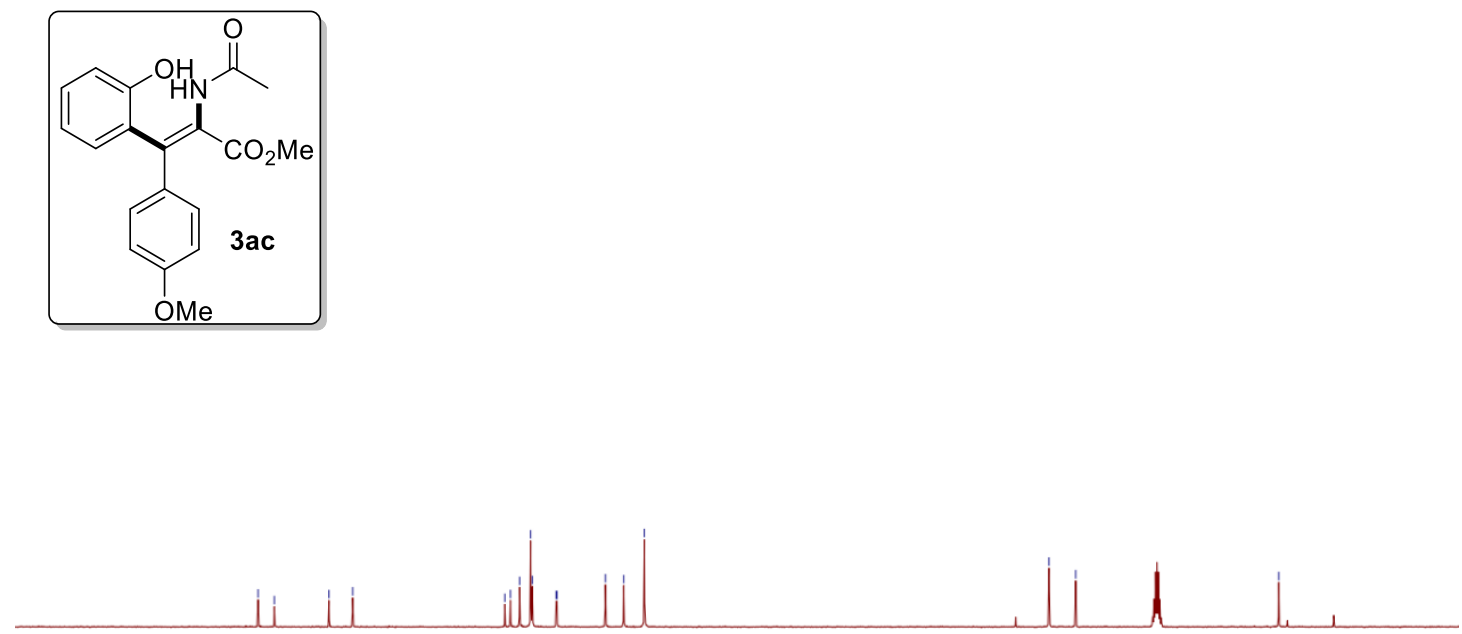

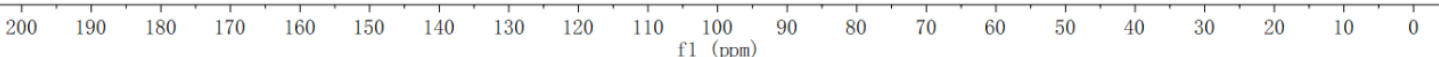


${ }^{1} \mathrm{H}{ }^{13} \mathrm{C}$ and ${ }^{19} \mathrm{~F}$ NMR of $\mathbf{3 a d}$

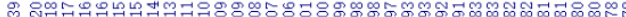

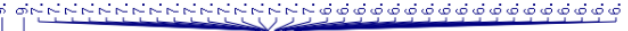
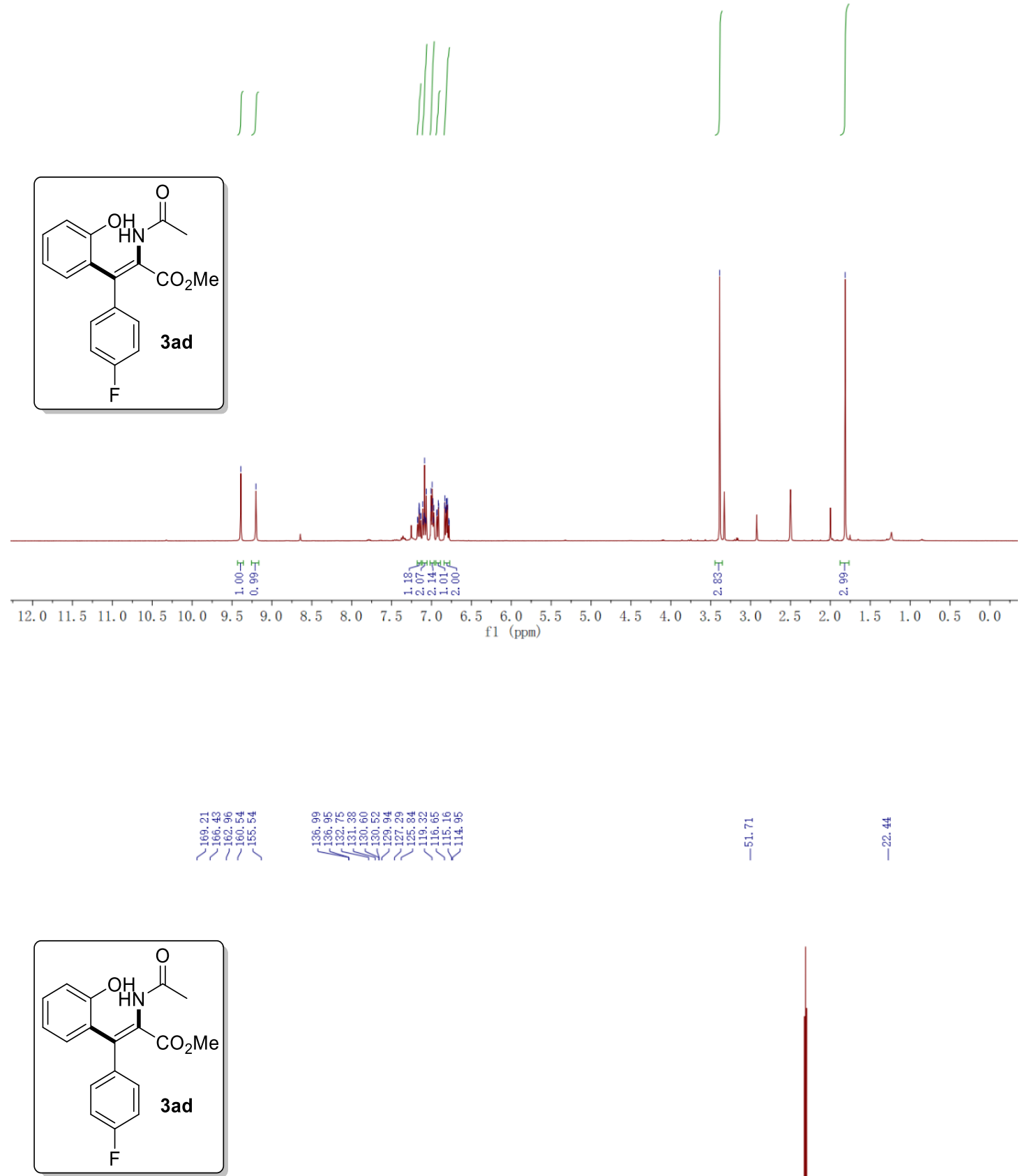

$\begin{array}{lllllllllll}200 & 190 & 180 & 170 & 160 & 150 & 140 & 130 & 120 & 110 & 100\end{array}$ 

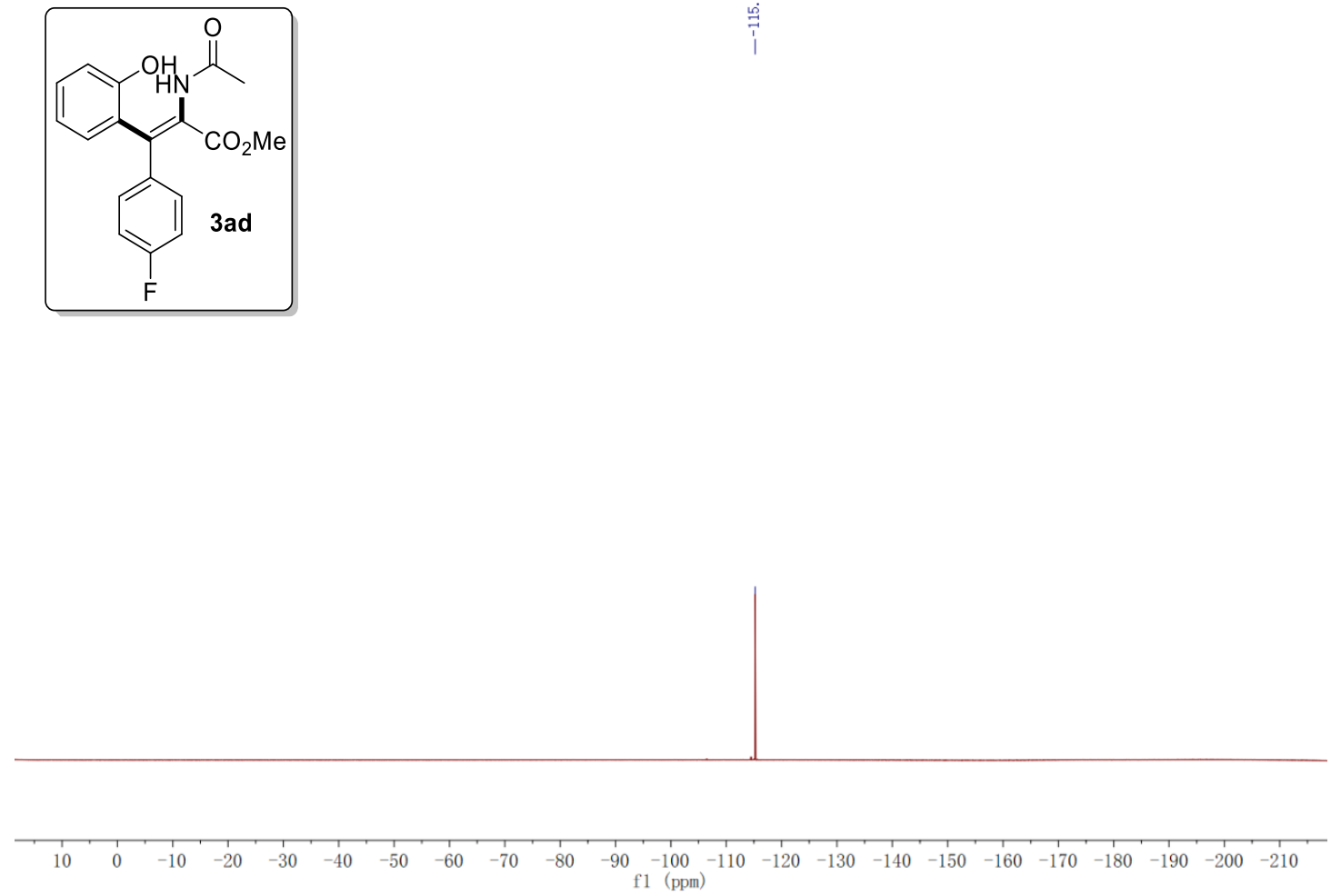
${ }^{1} \mathrm{H}$ and ${ }^{13} \mathrm{C}$ NMR of $\mathbf{3 a e}$

유씨씼

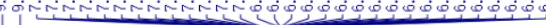
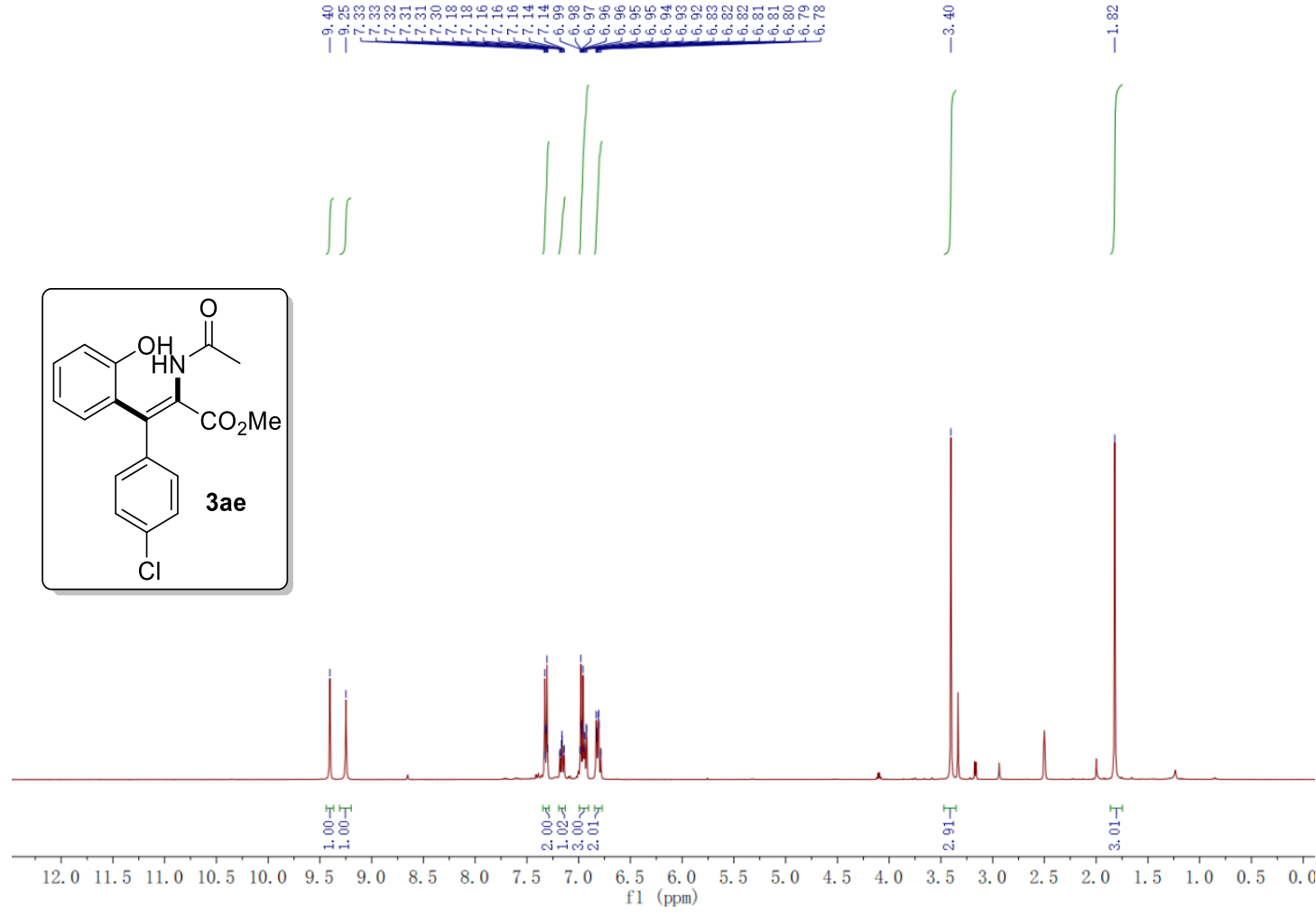

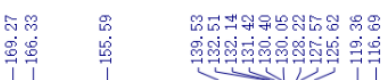

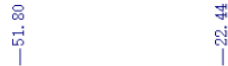
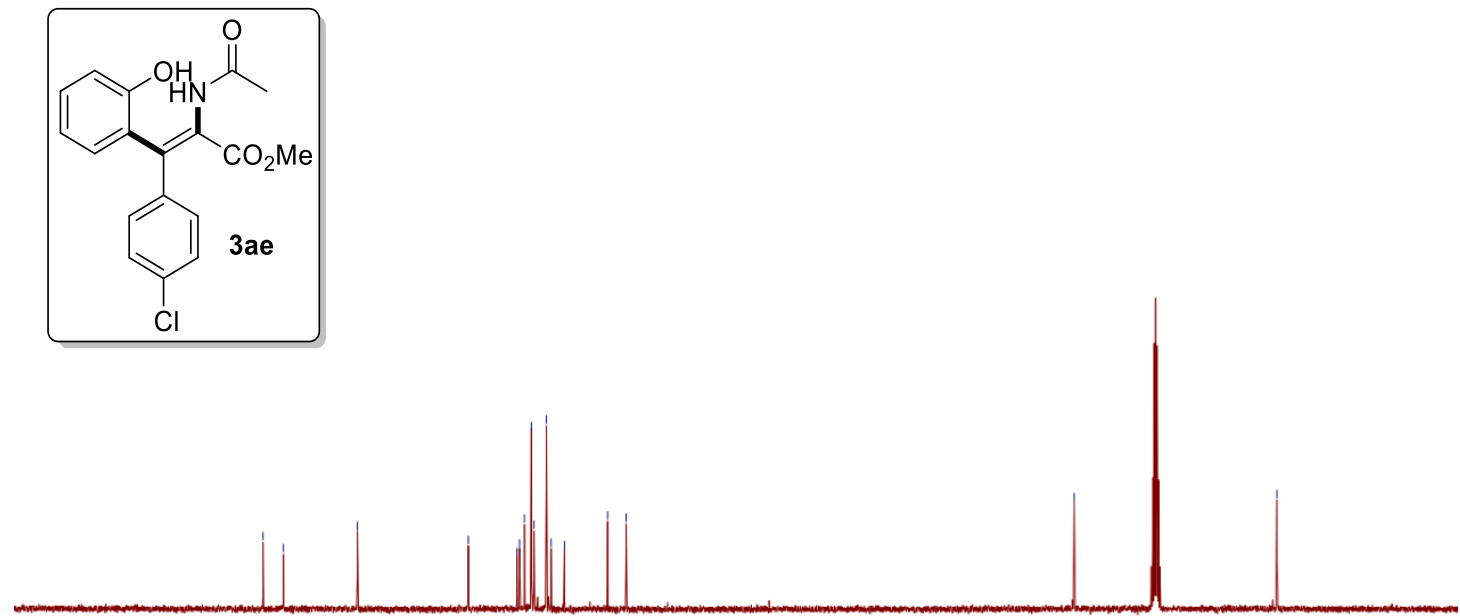

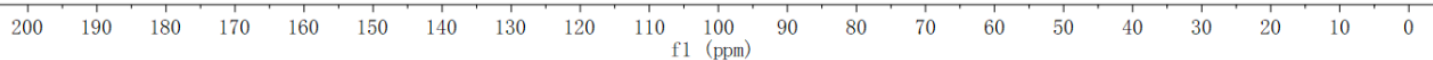


${ }^{1} \mathrm{H}$ and ${ }^{13} \mathrm{C}$ NMR of $\mathbf{3 a f}$
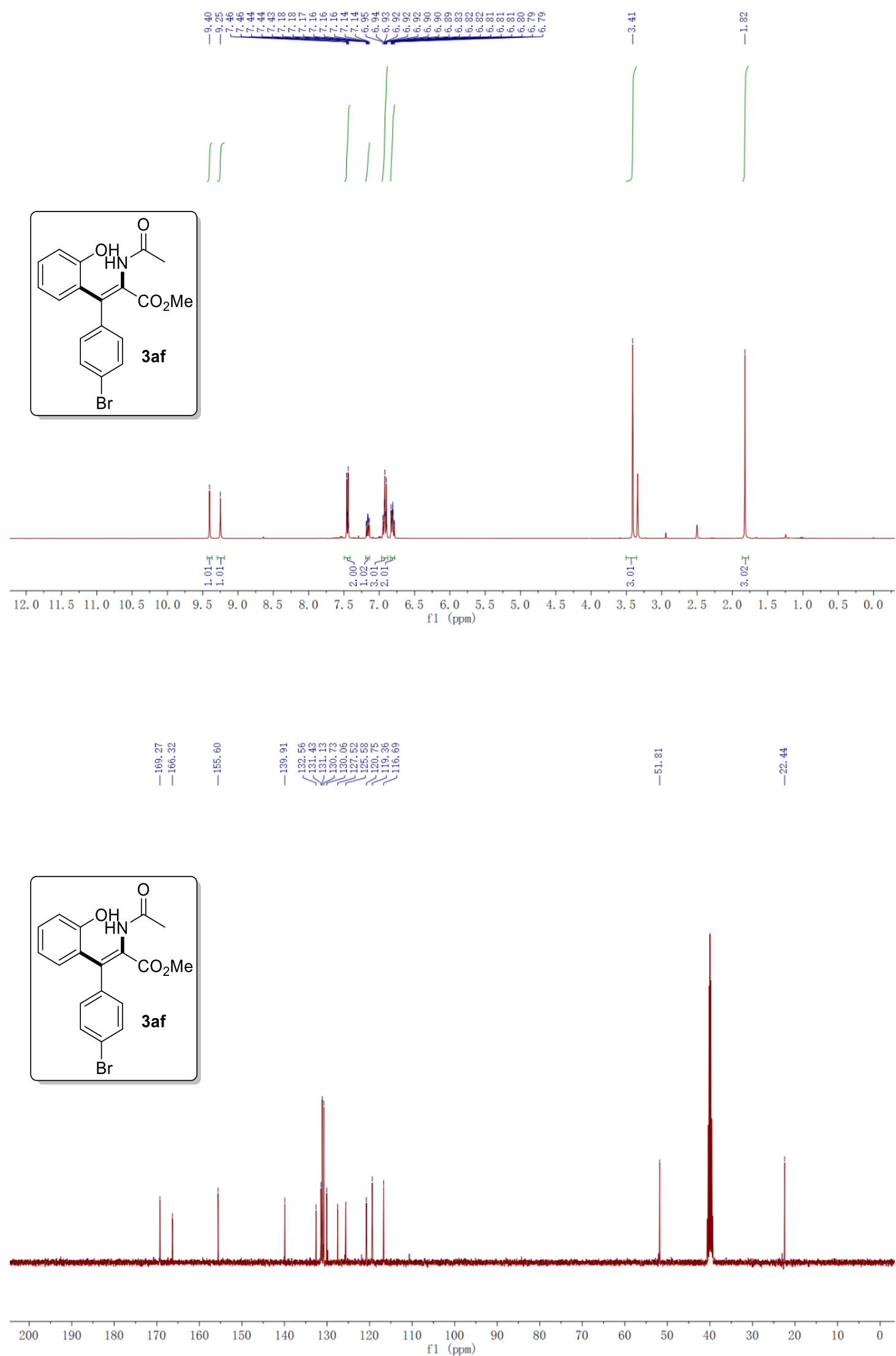
${ }^{1} \mathrm{H}$ and ${ }^{13} \mathrm{C}$ NMR of $\mathbf{3 a g}$
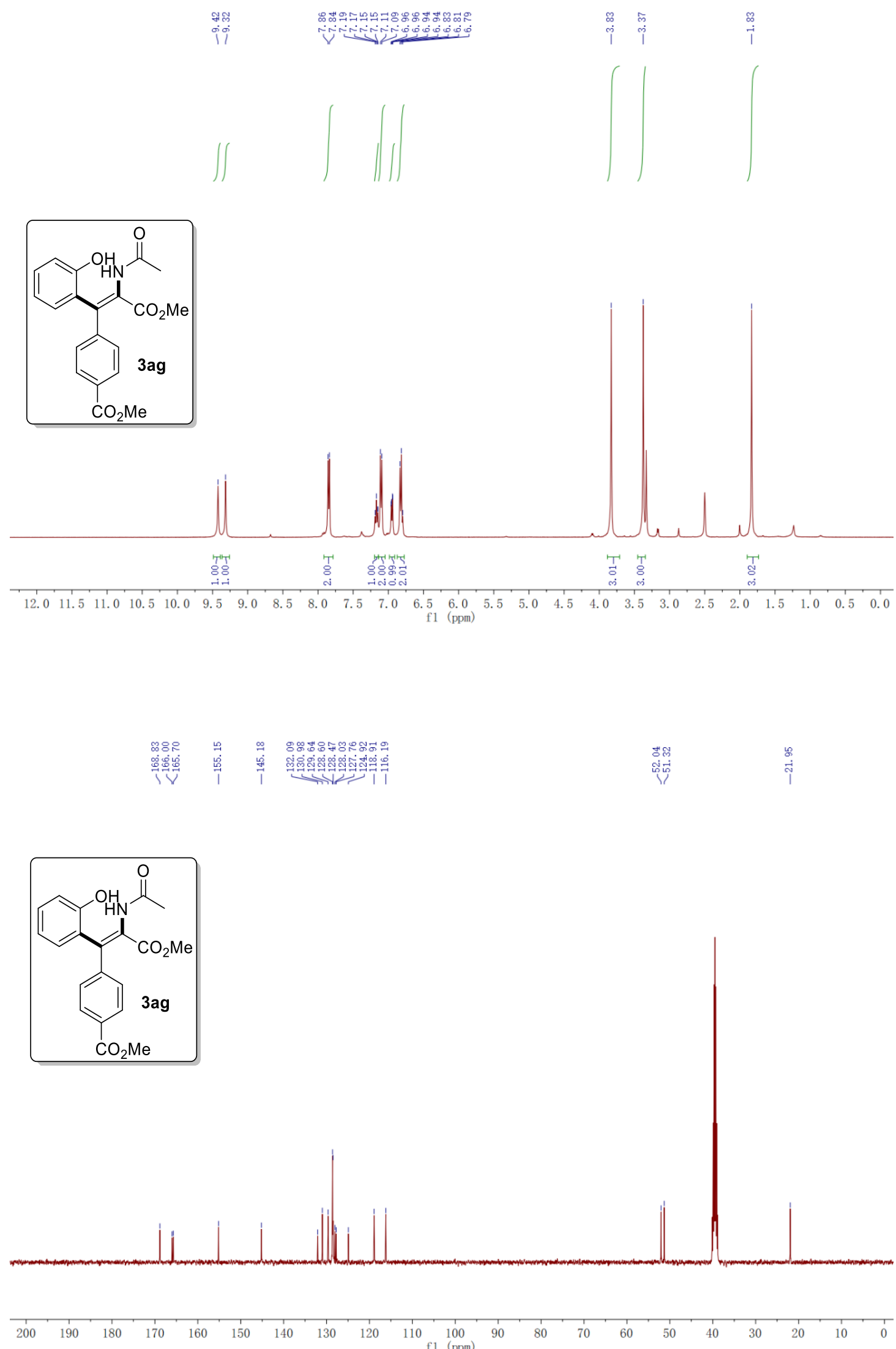


\section{${ }^{1} \mathrm{H}$ and ${ }^{13} \mathrm{C}$ NMR of $\mathbf{3 a h}$}

息
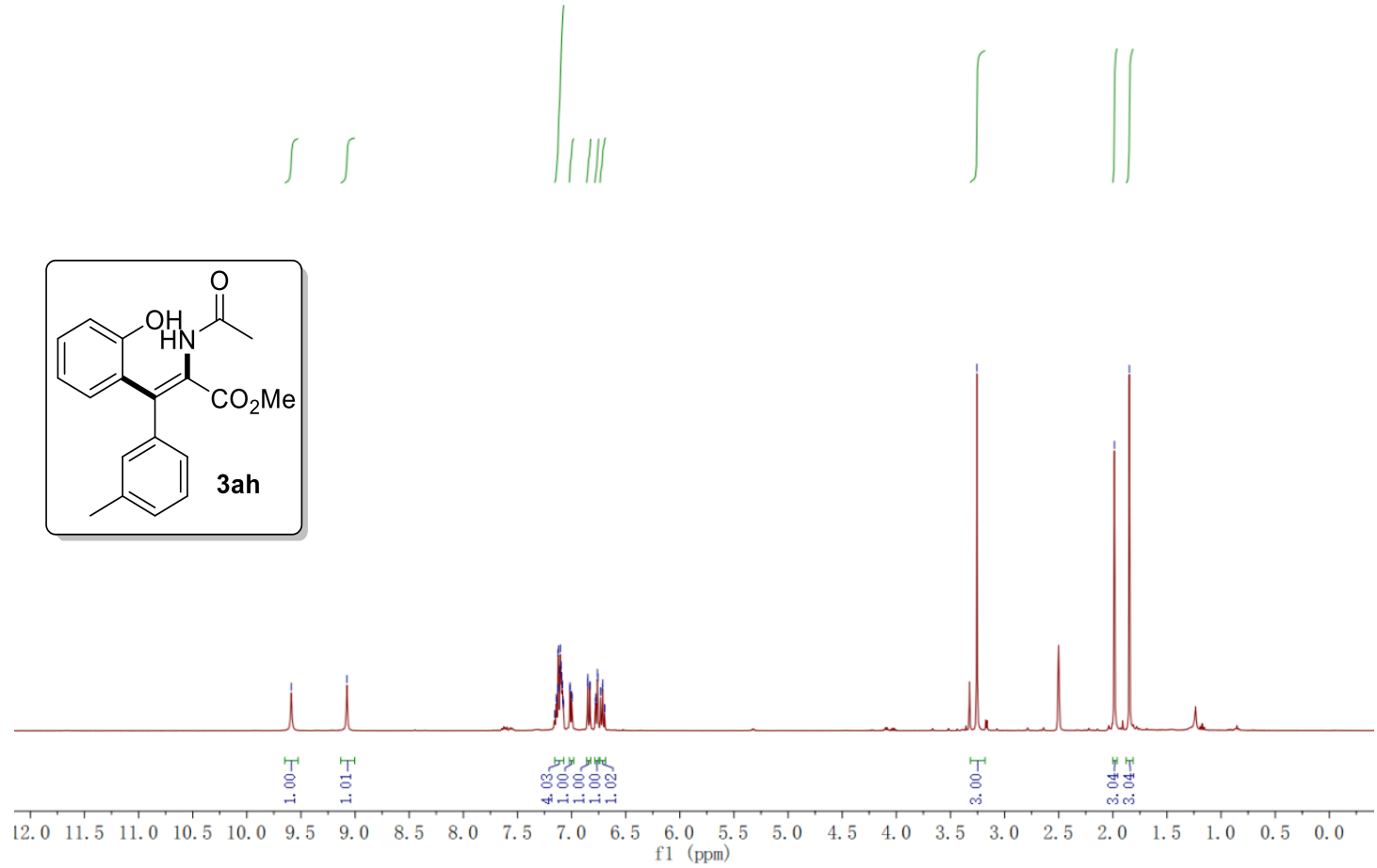

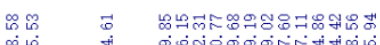

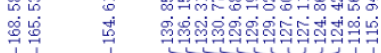
(
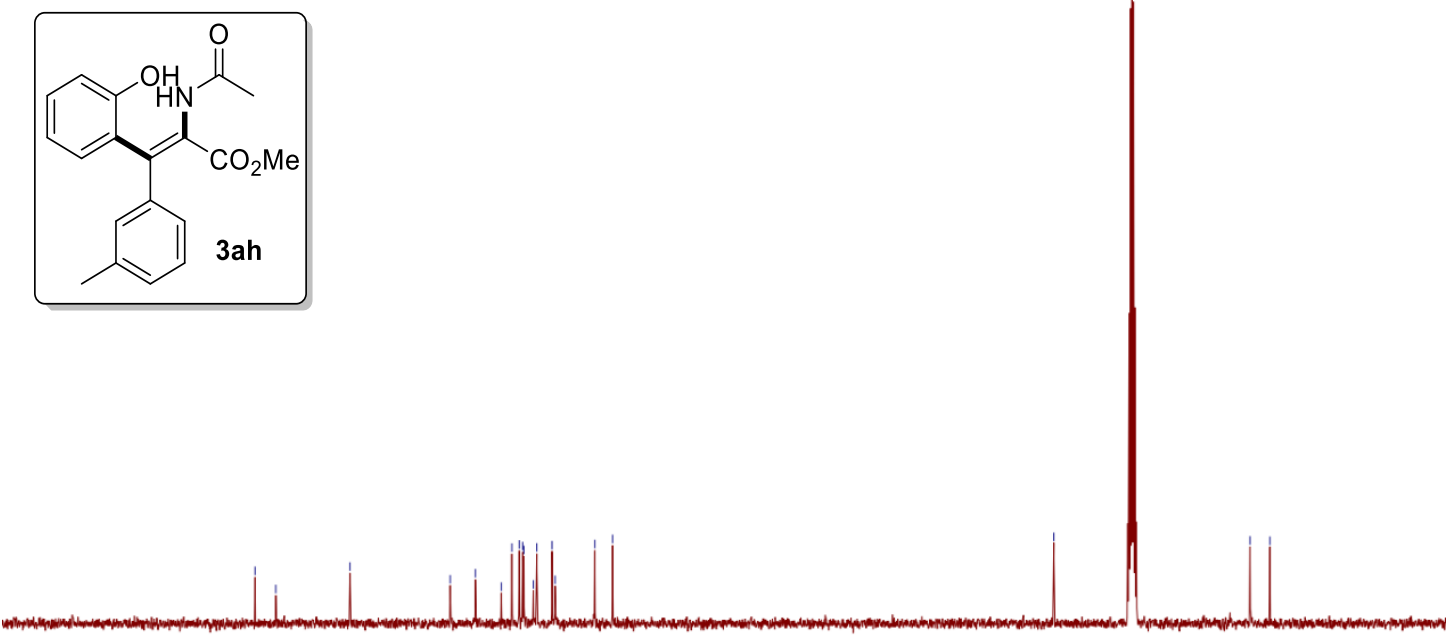

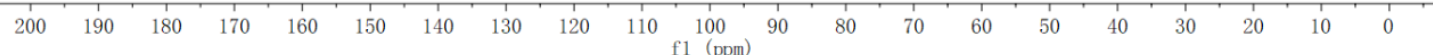


${ }^{1} \mathrm{H}$ and ${ }^{13} \mathrm{C}$ NMR of 3ai
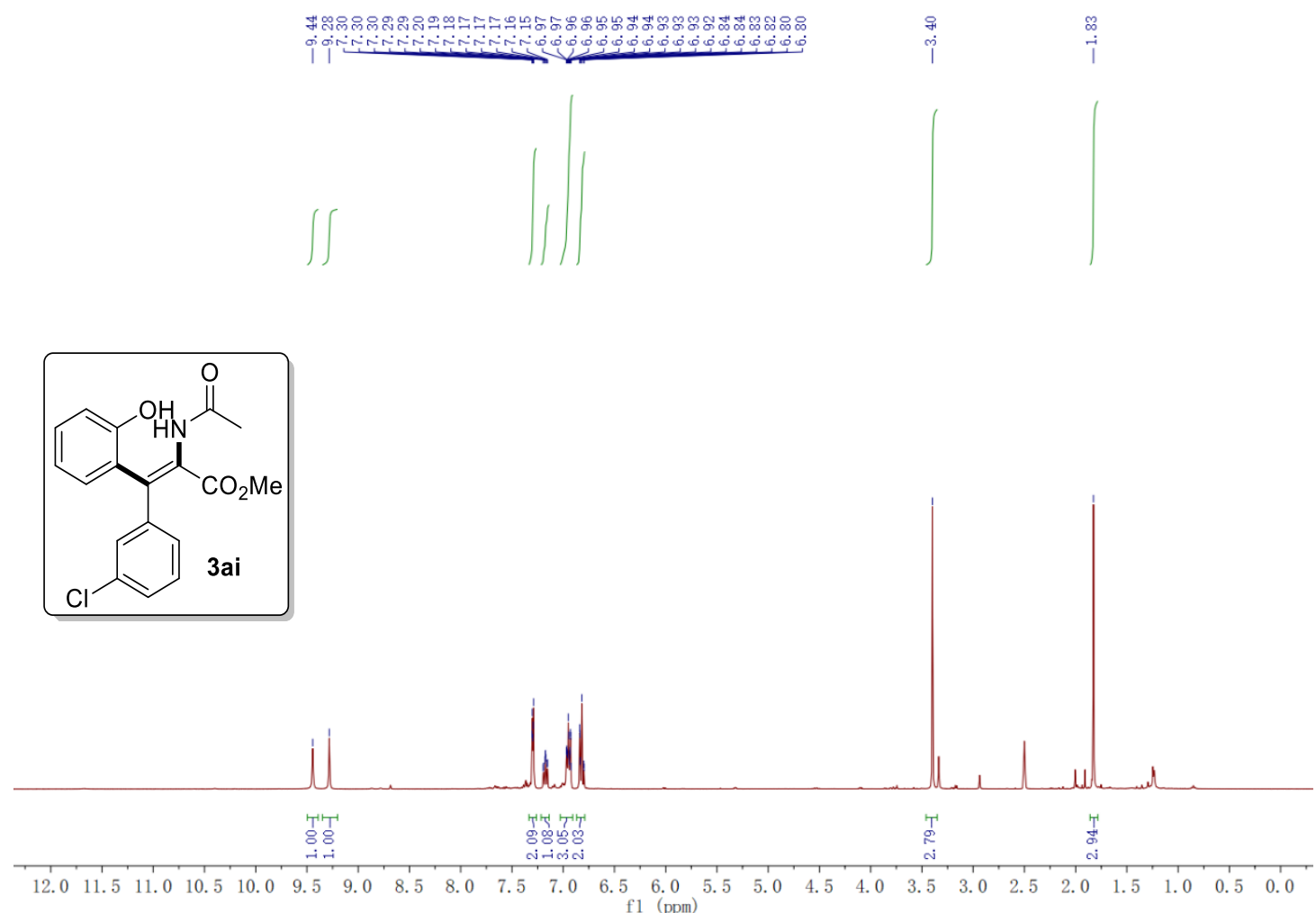

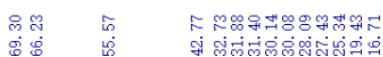

| ।

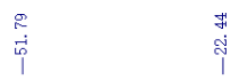
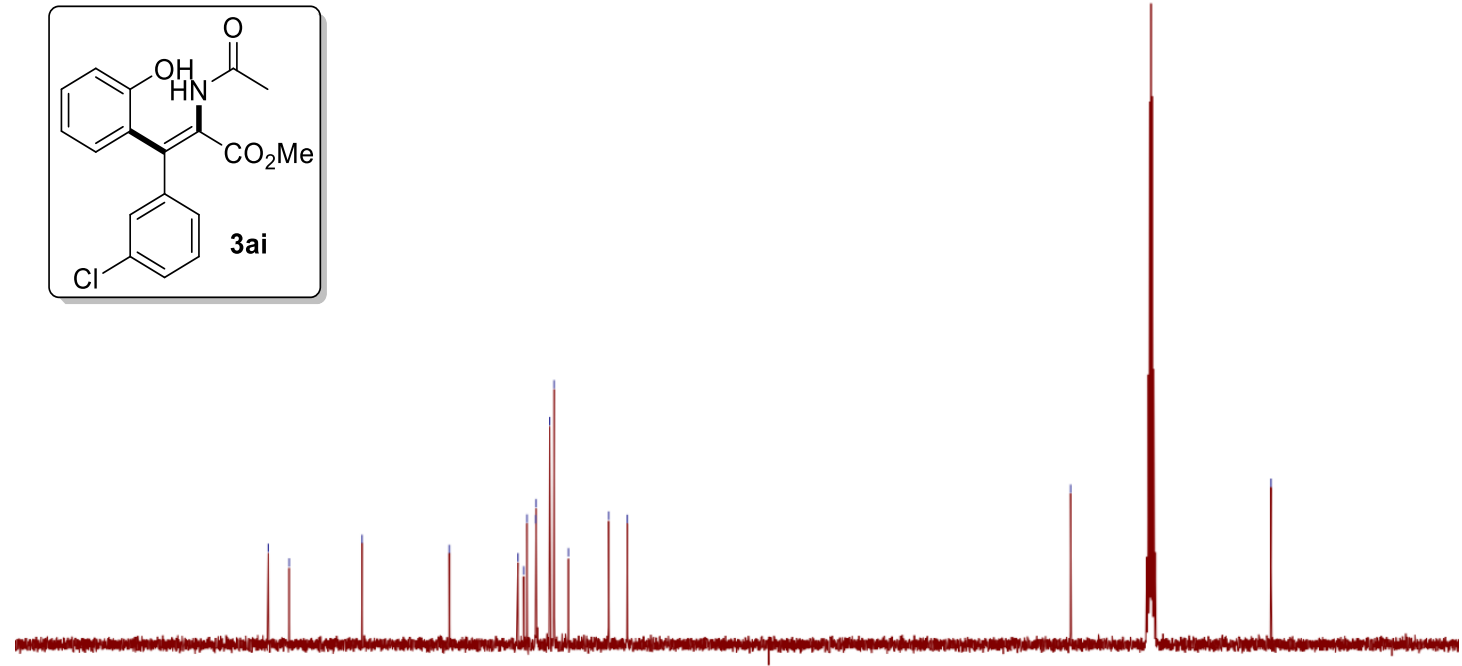

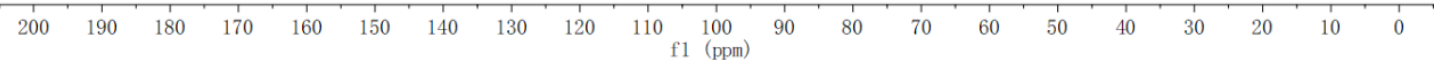


${ }^{1} \mathrm{H}$ and ${ }^{13} \mathrm{C}$ NMR of $\mathbf{3 a j}$

o
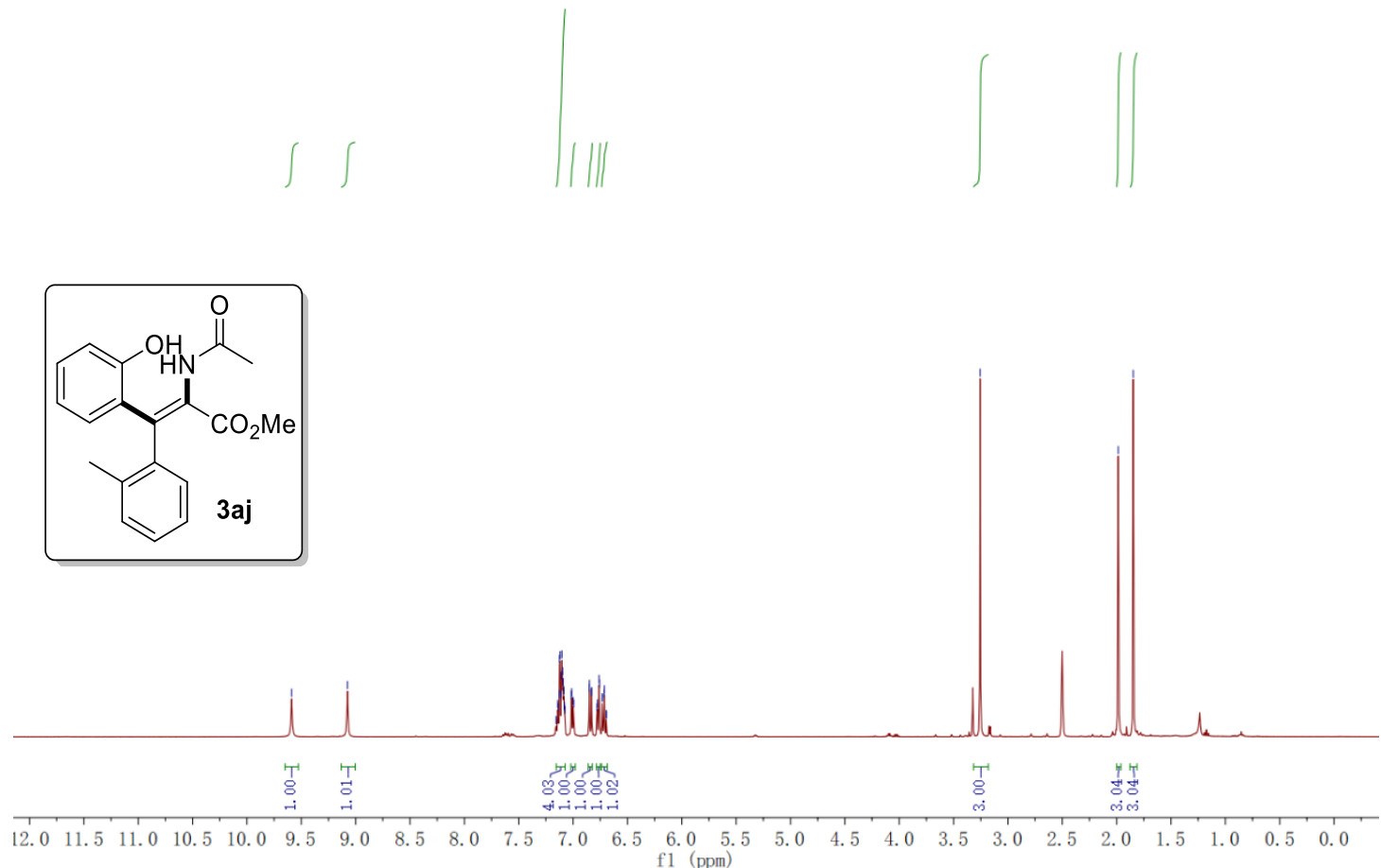

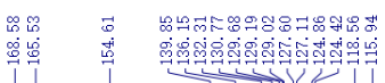
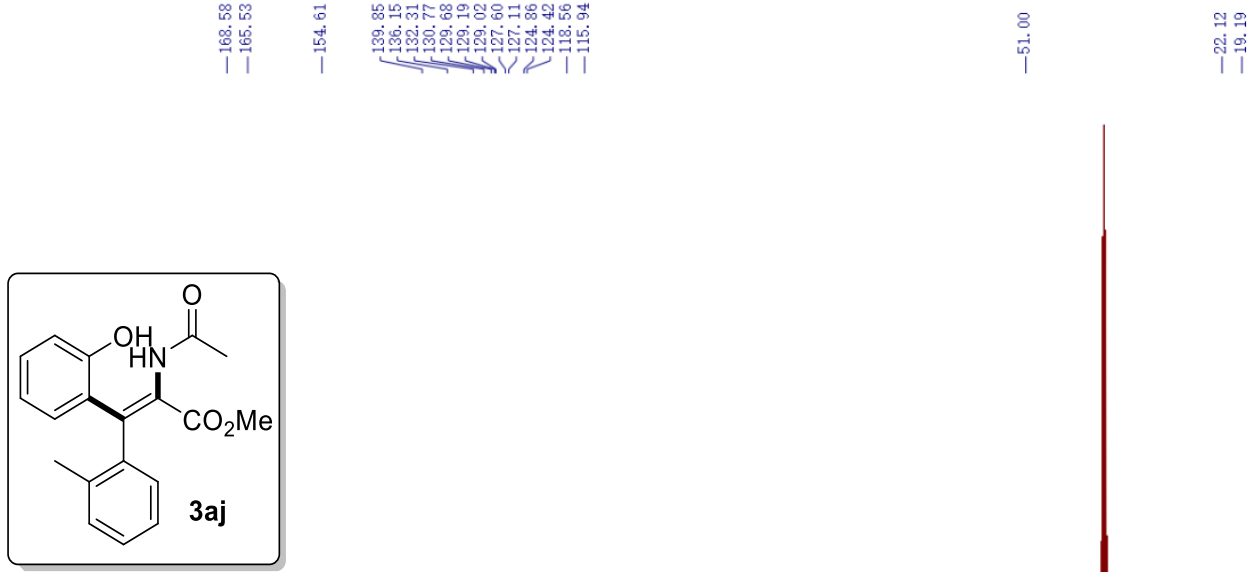

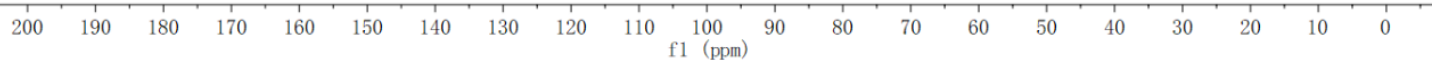


${ }^{1} \mathrm{H}{ }^{13} \mathrm{C}$ and ${ }^{19} \mathrm{~F}$ NMR of $\mathbf{3 a k}$

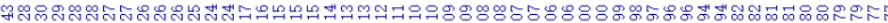

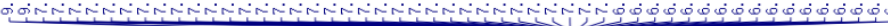

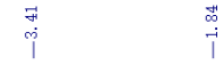

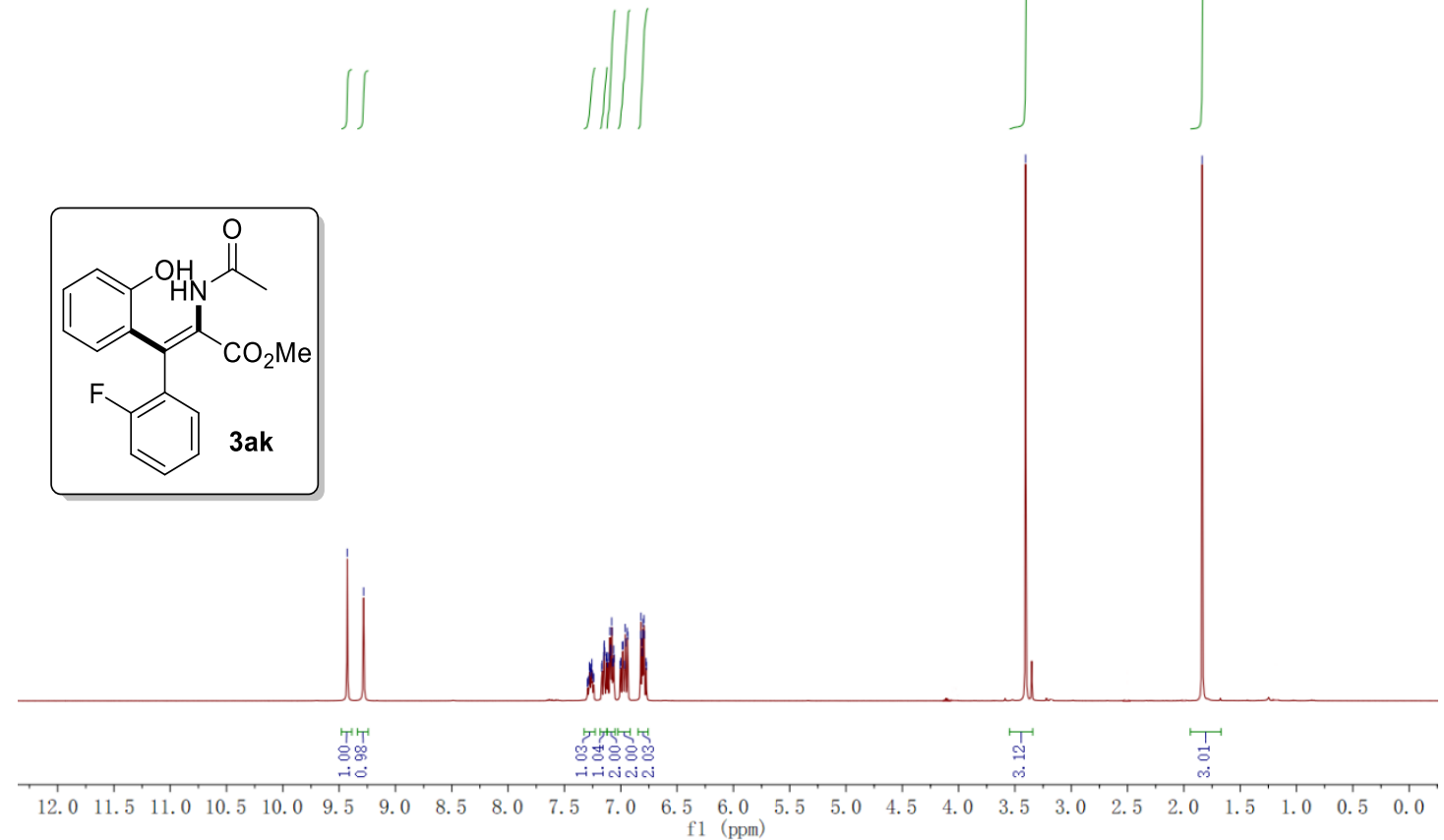

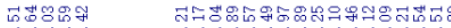

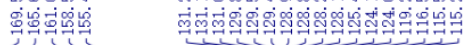
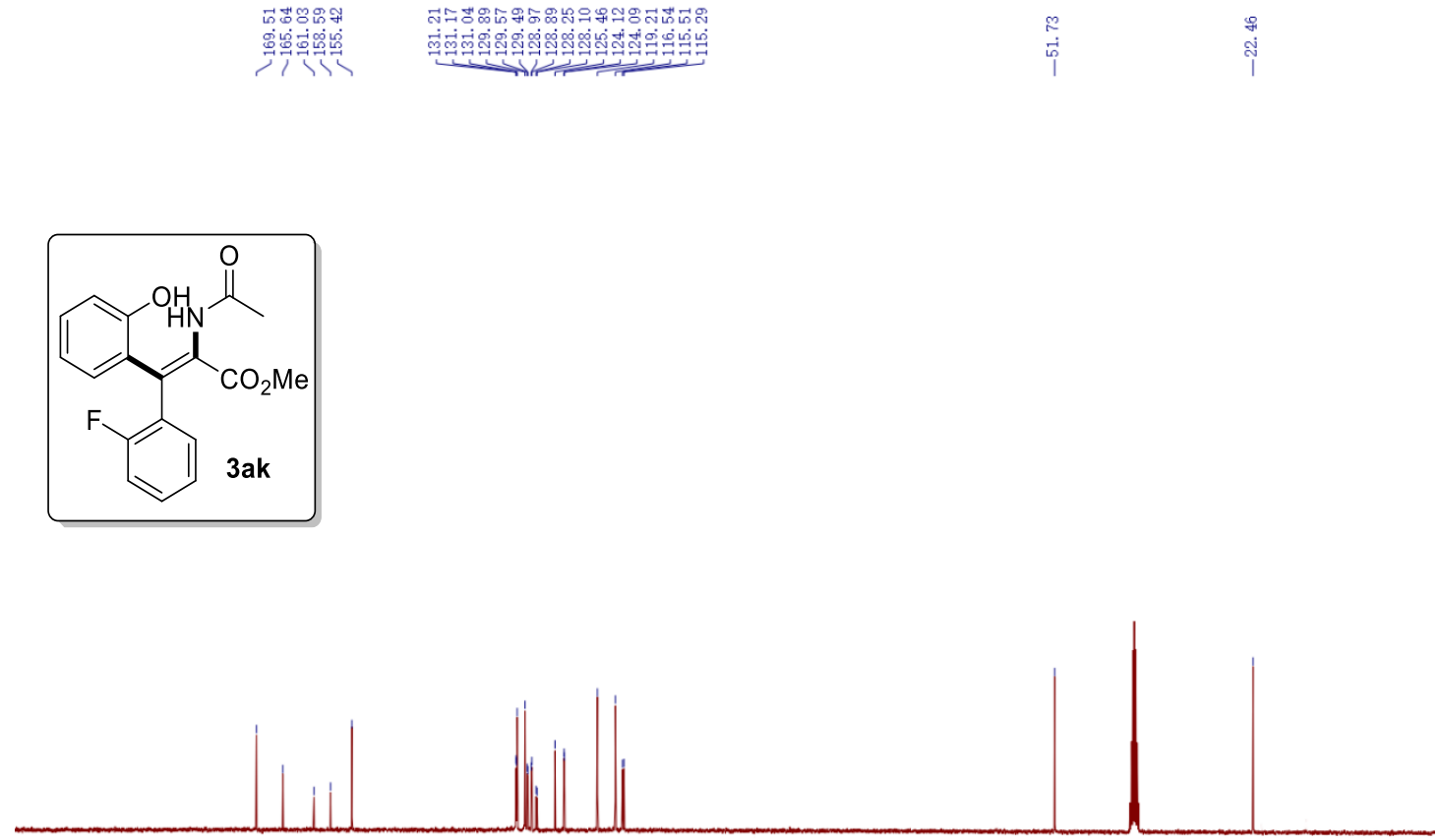

$\begin{array}{lllllllllllllllllllll}200 & 190 & 180 & 170 & 160 & 150 & 140 & 130 & 120 & 110 & 100 & 90 & 80 & 70 & 60 & 50 & 40 & 30 & 20 & 10 & 0\end{array}$ 


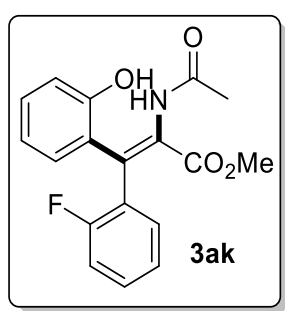

辛

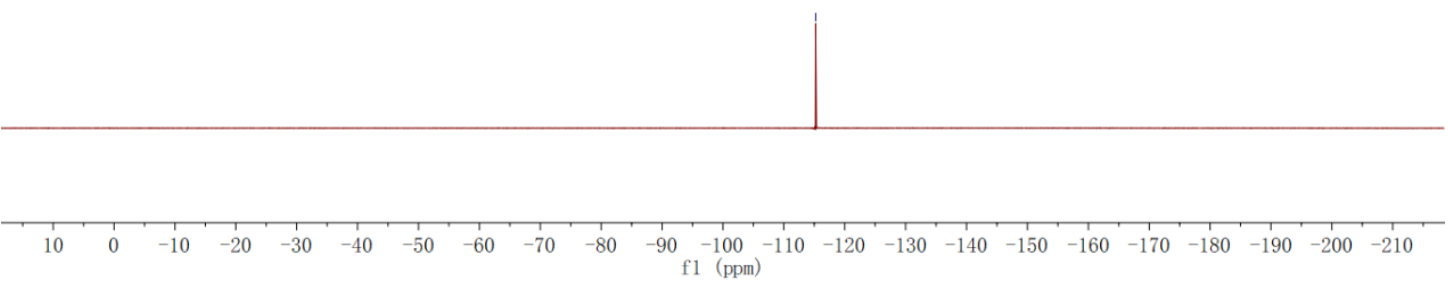


${ }^{1} \mathrm{H}$ and ${ }^{13} \mathrm{C}$ NMR of 3al

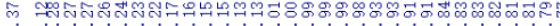

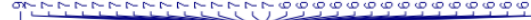

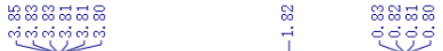
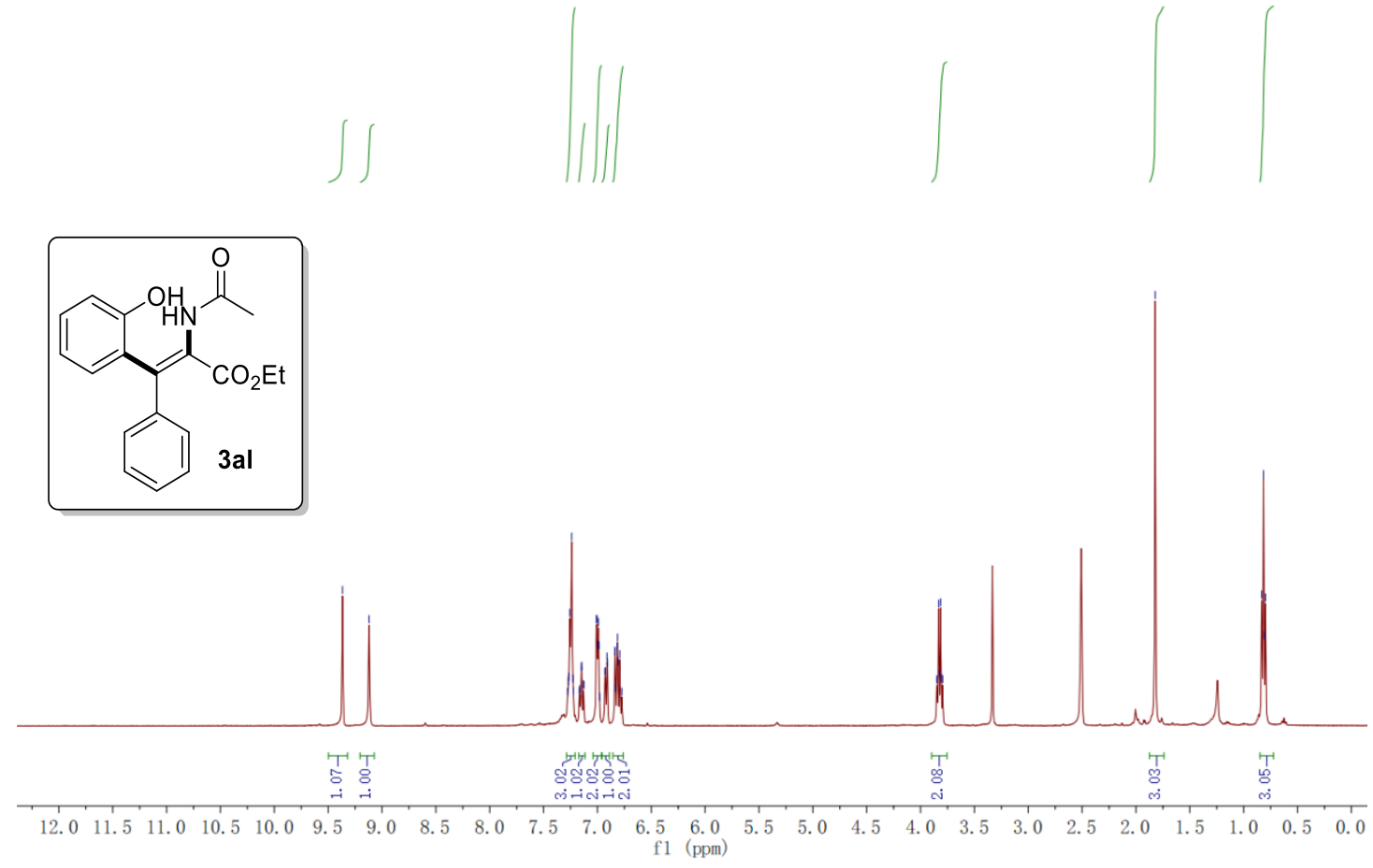

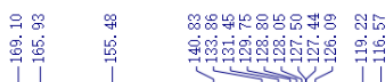
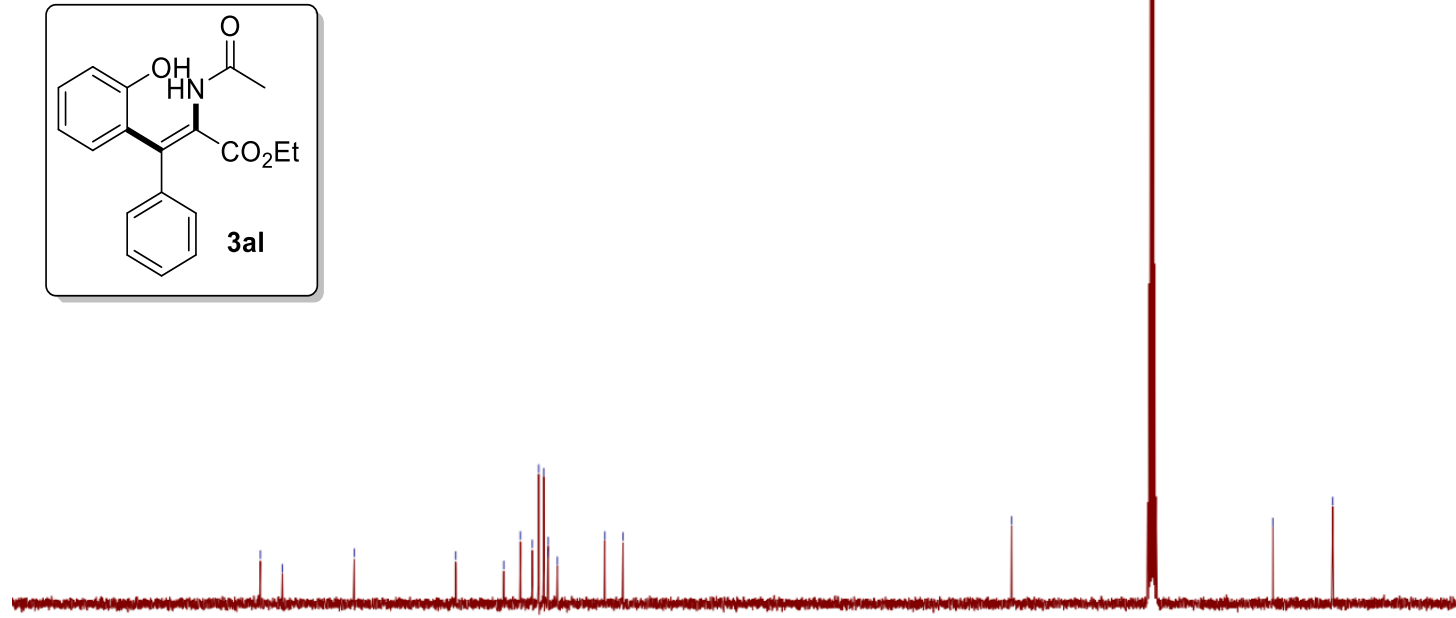

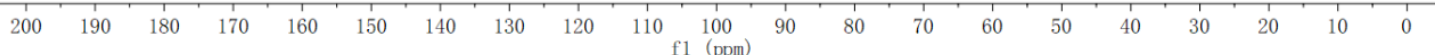


${ }^{1} \mathrm{H}$ and ${ }^{13} \mathrm{C}$ NMR of $\mathbf{3 a m}$

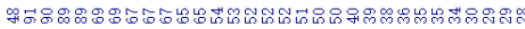

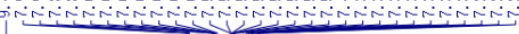
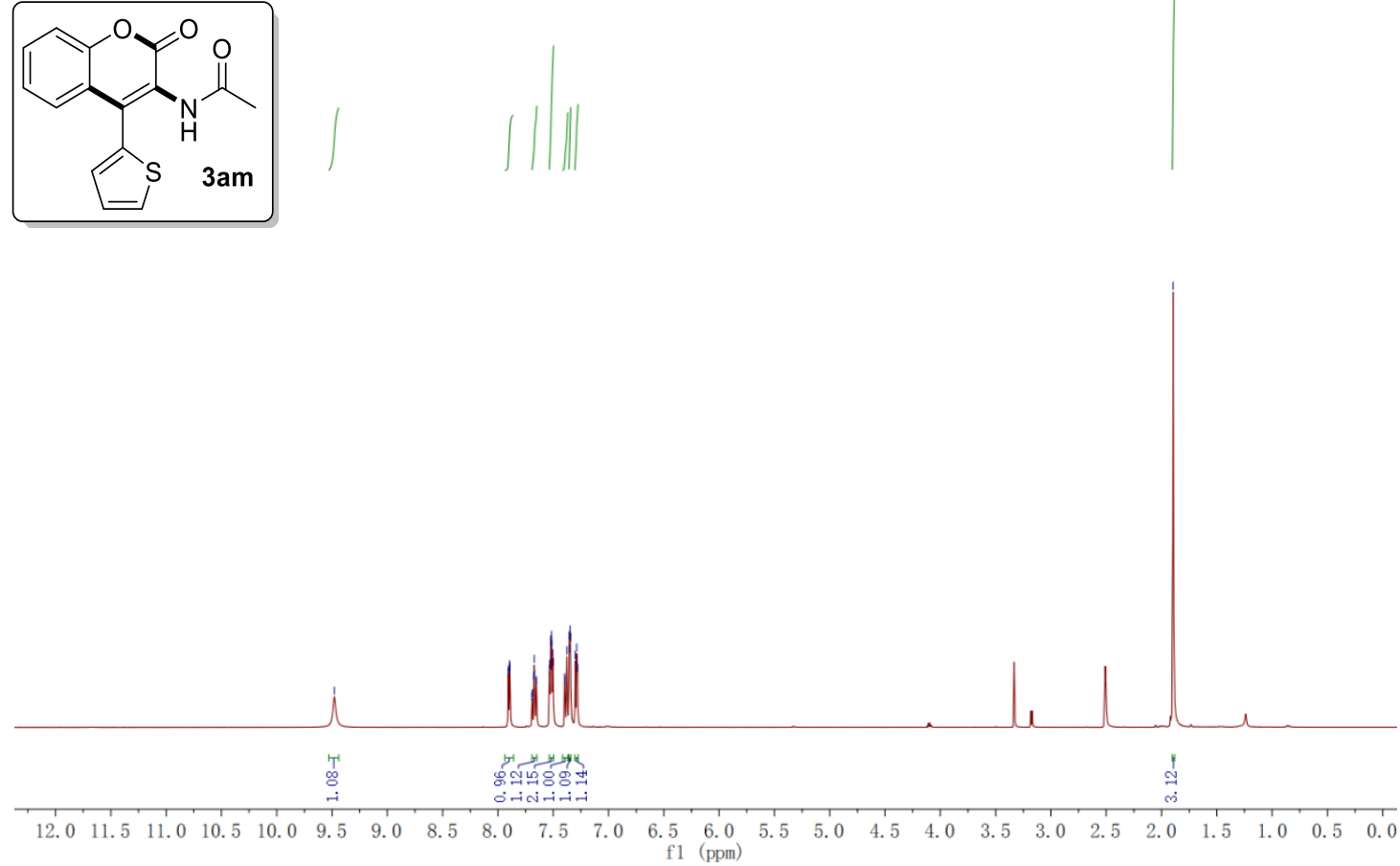

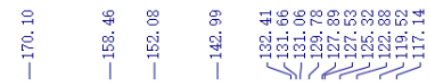
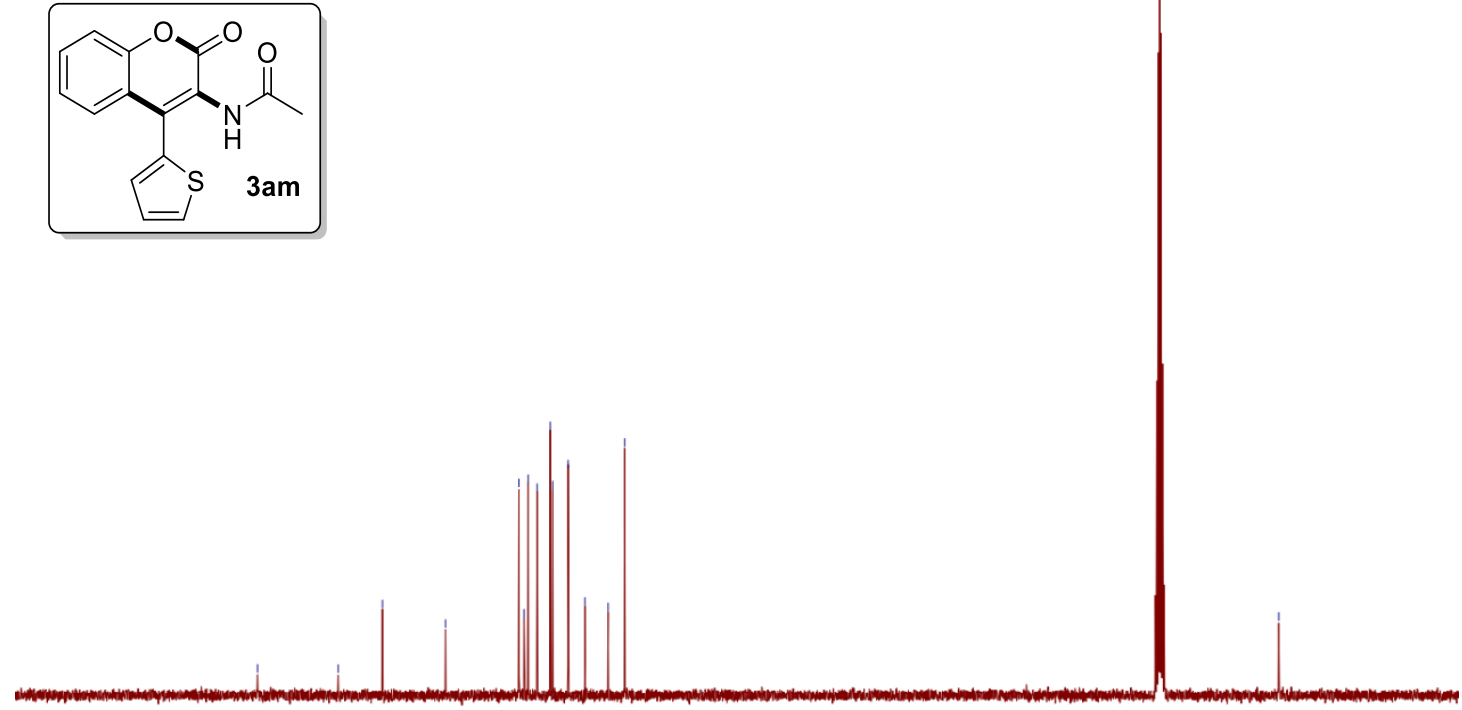

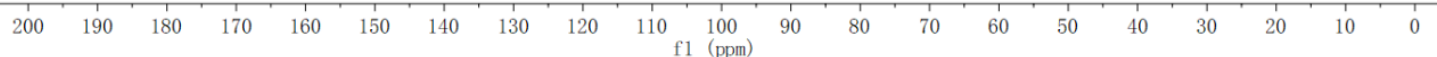


${ }^{1} \mathrm{H}$ and ${ }^{13} \mathrm{C}$ NMR of 3an

$\begin{array}{ll}\overrightarrow{0} & 0 \\ 0 & 1\end{array}$

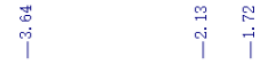

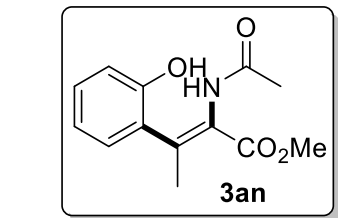

$\int \| \int$

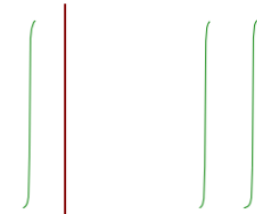

Hill

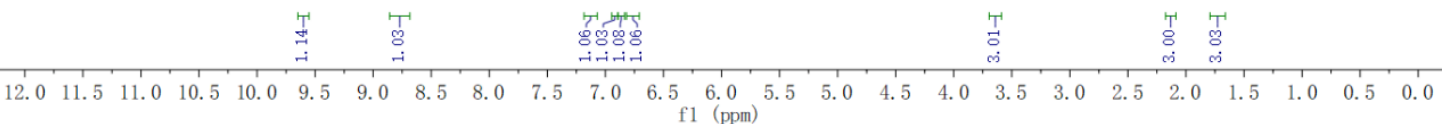

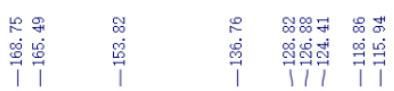

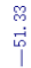

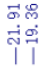

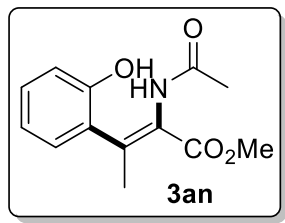

an

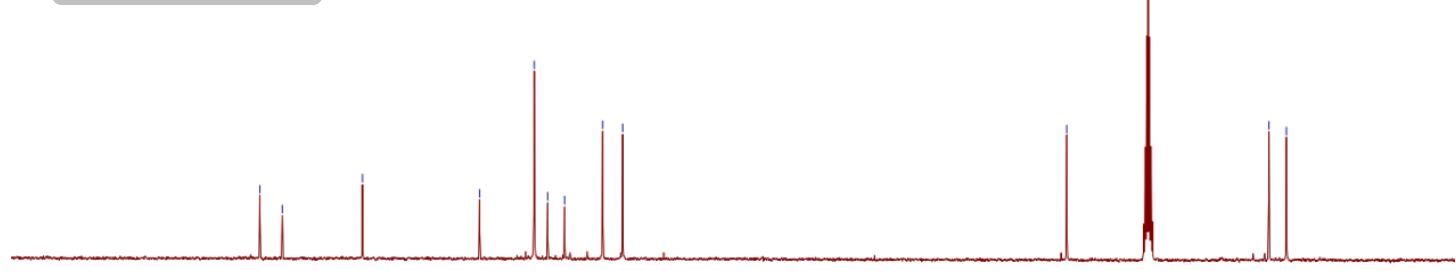

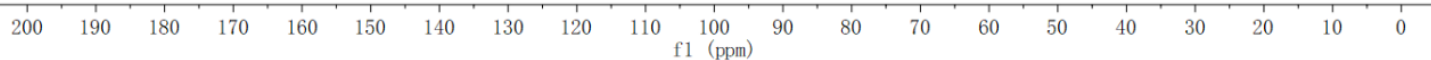


${ }^{1} \mathrm{H}$ and ${ }^{13} \mathrm{C}$ NMR of $3 \mathbf{a o}$

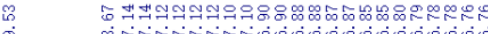

10

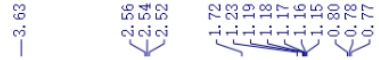

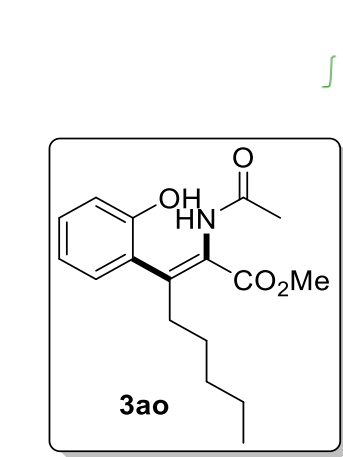

$\iint$
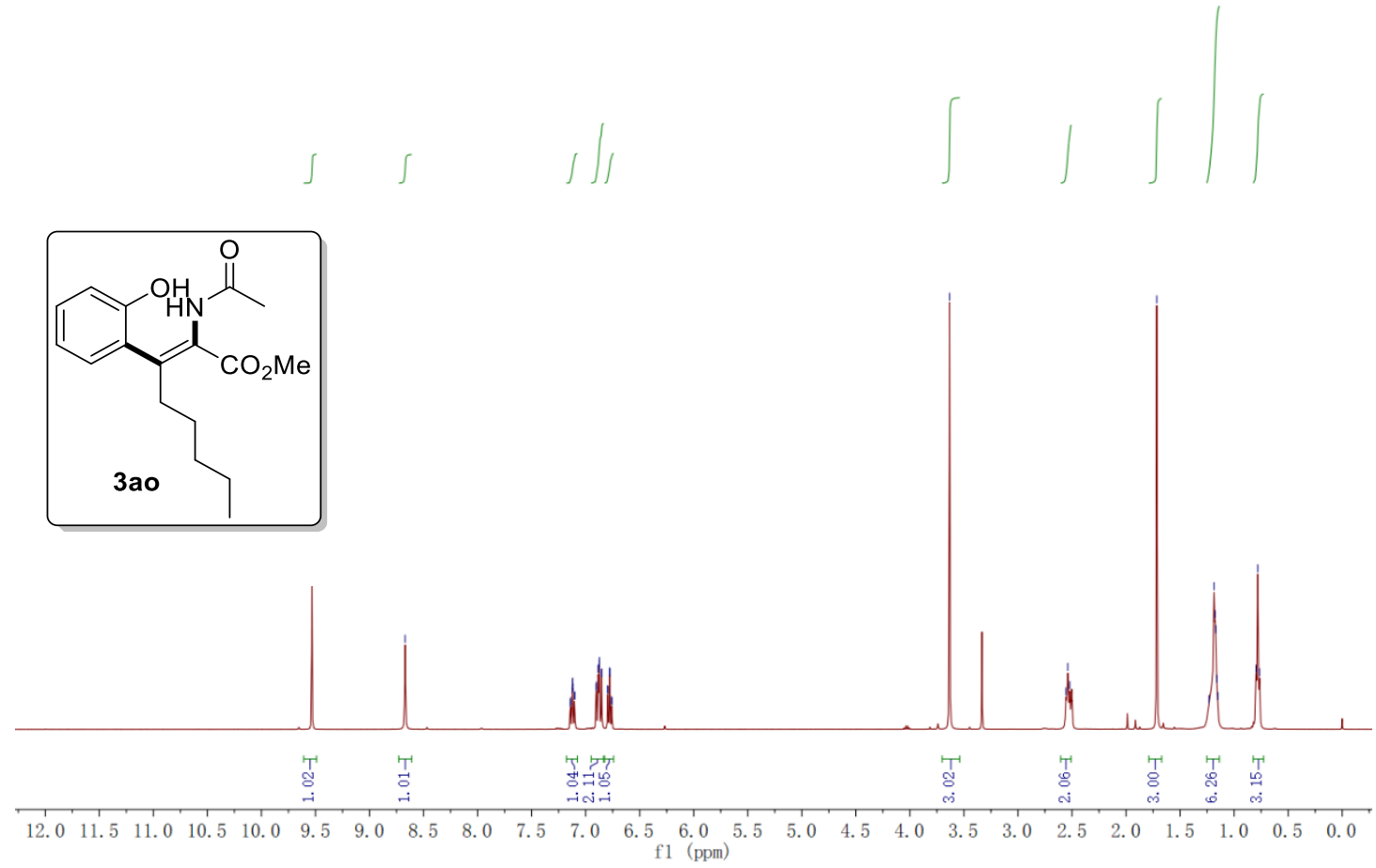

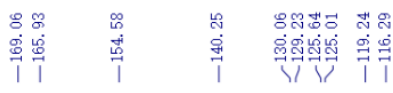

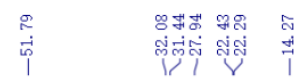
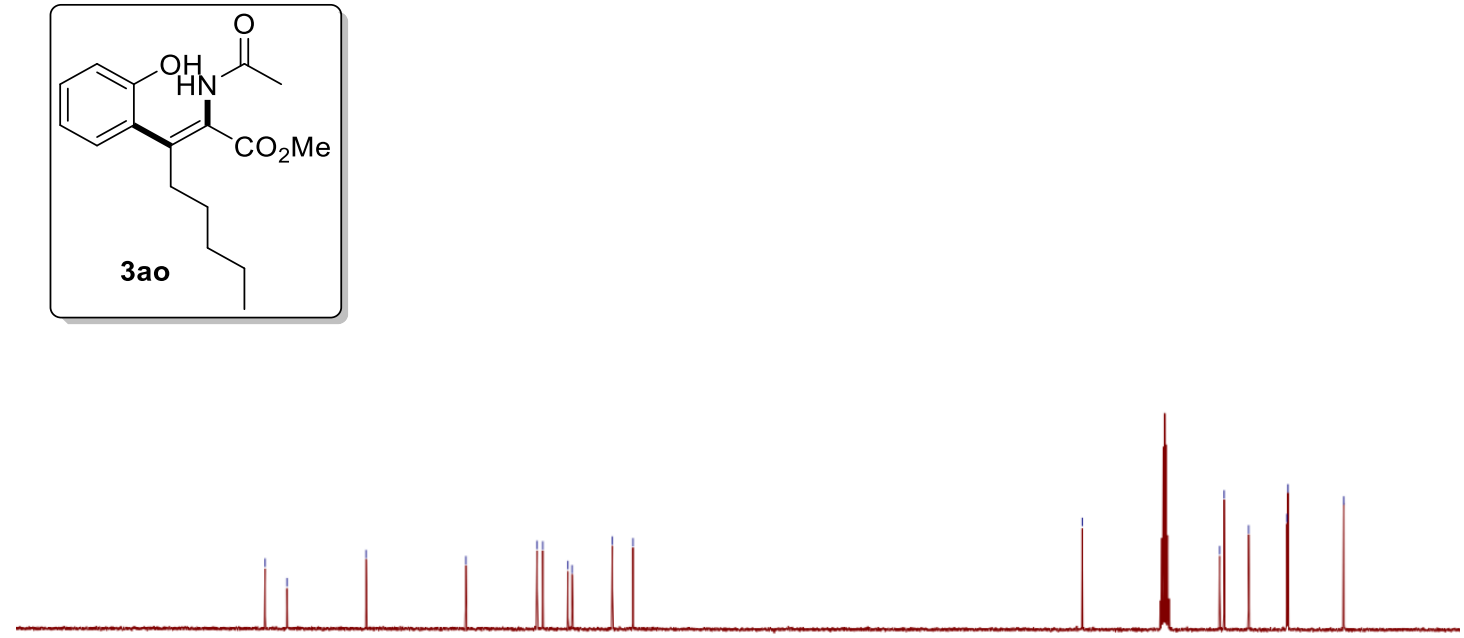

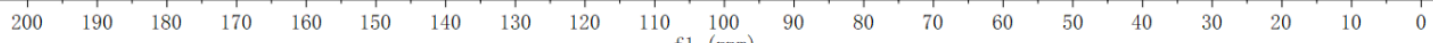




\section{${ }^{1} \mathrm{H}$ and ${ }^{13} \mathrm{C}$ NMR of $\mathbf{5 a a}$}

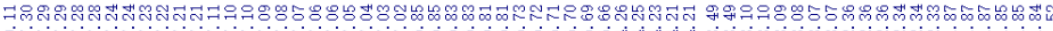

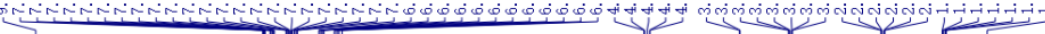

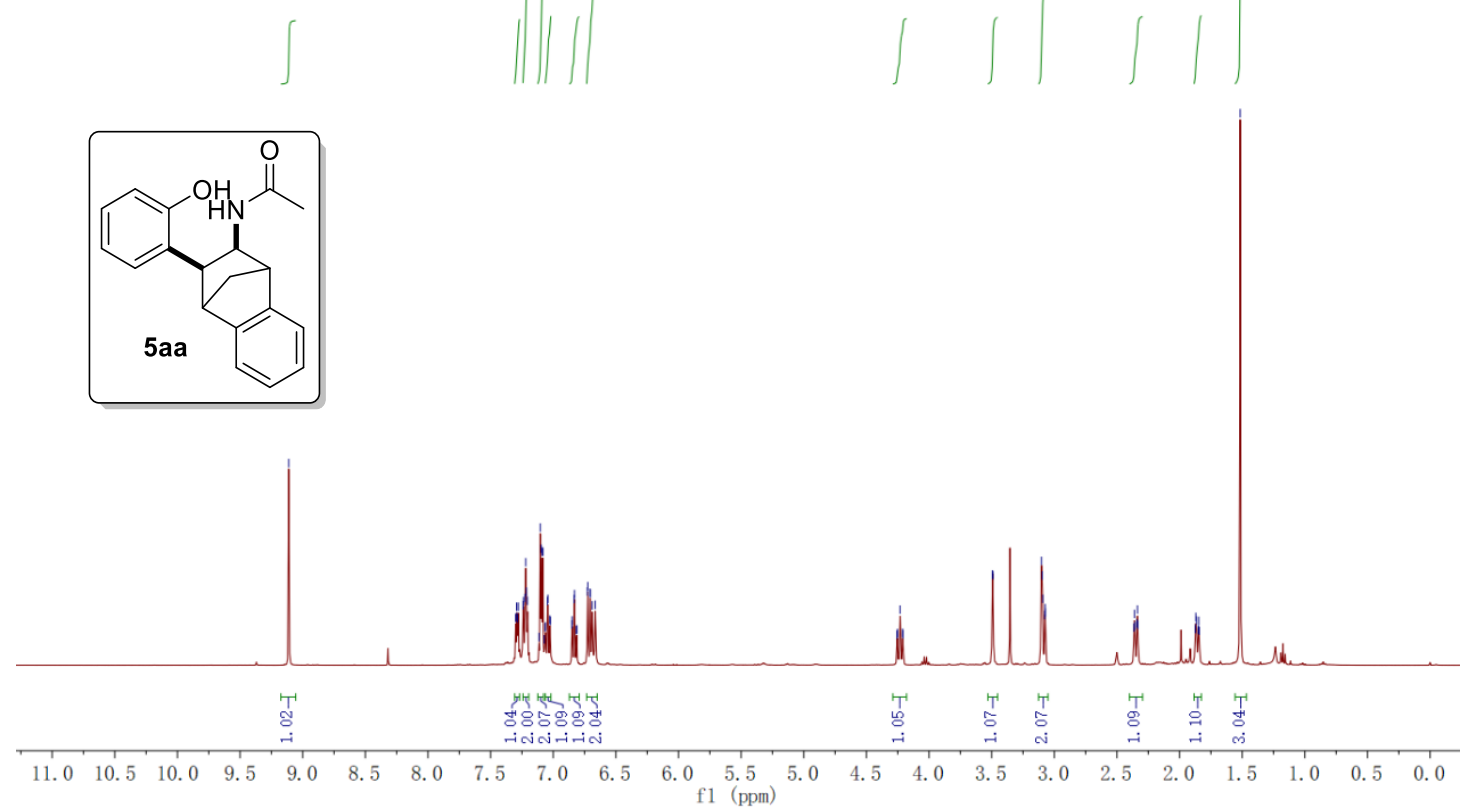

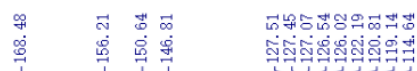

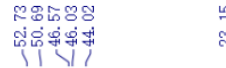
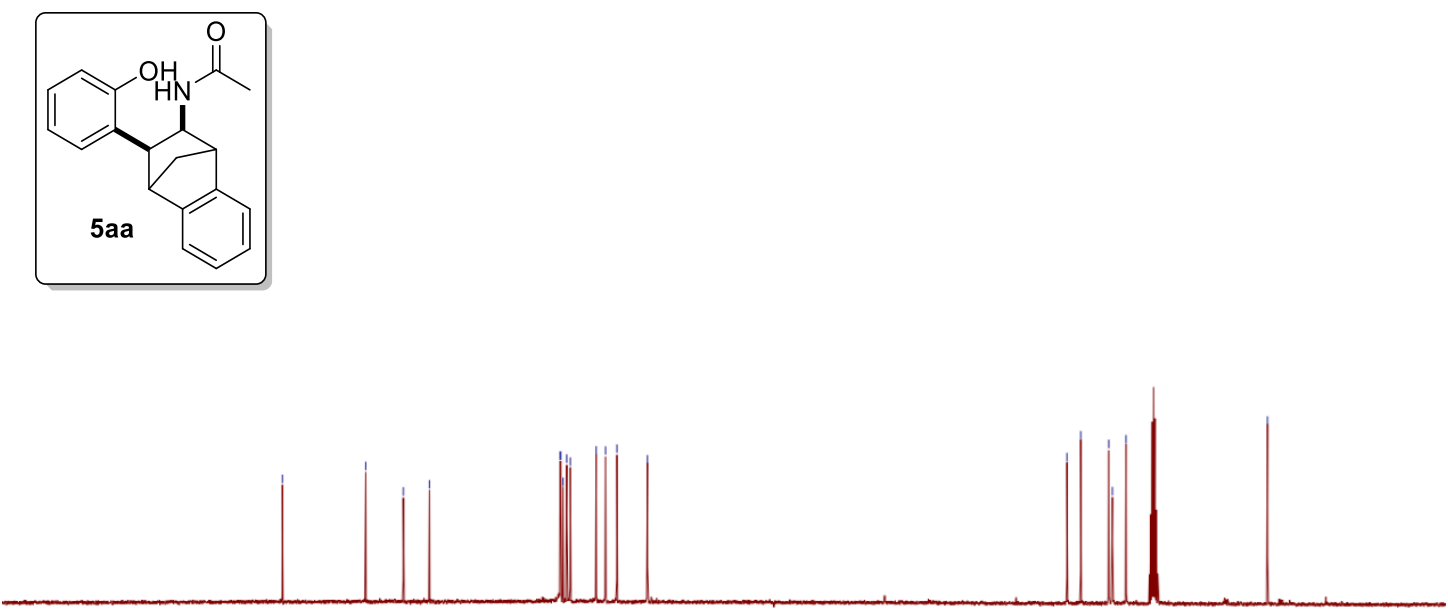

$\begin{array}{lllllllllllllllllllllll}200 & 190 & 180 & 170 & 160 & 150 & 140 & 130 & 120 & 110 & 100 & 90 & 80 & 70 & 60 & 50 & 40 & 30 & 20 & 10 & 0\end{array}$ 
${ }^{1} \mathrm{H}$ and ${ }^{13} \mathrm{C}$ NMR of $\mathbf{5 b a}$

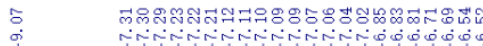

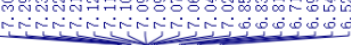

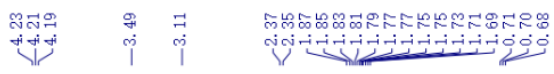
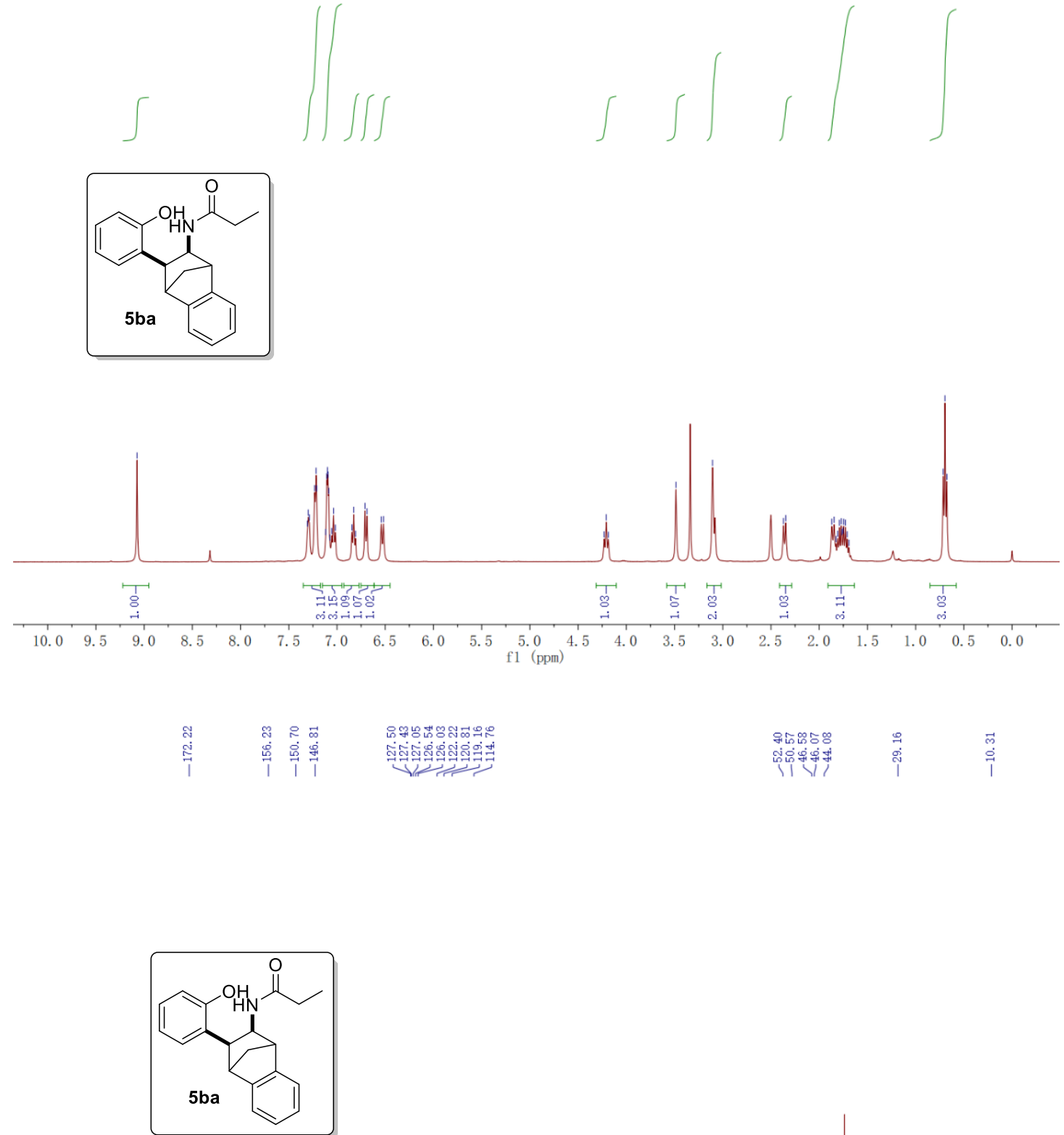

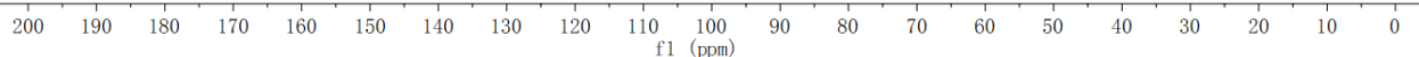




\section{${ }^{1} \mathrm{H}$ and ${ }^{13} \mathrm{C}$ NMR of $\mathbf{5 c a}$}

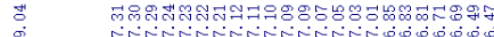
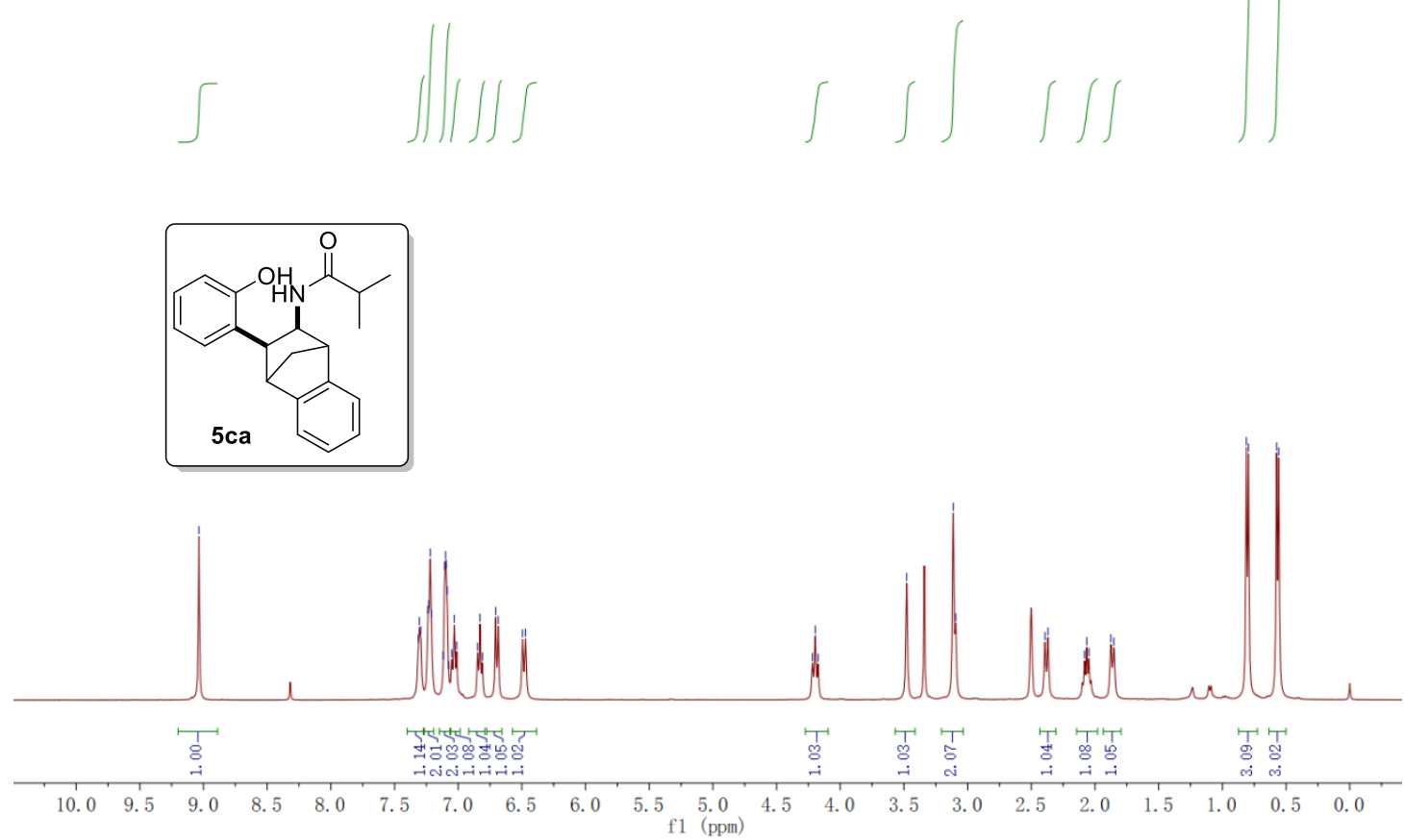

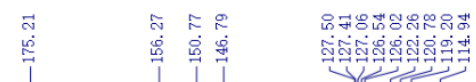

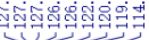

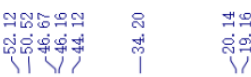
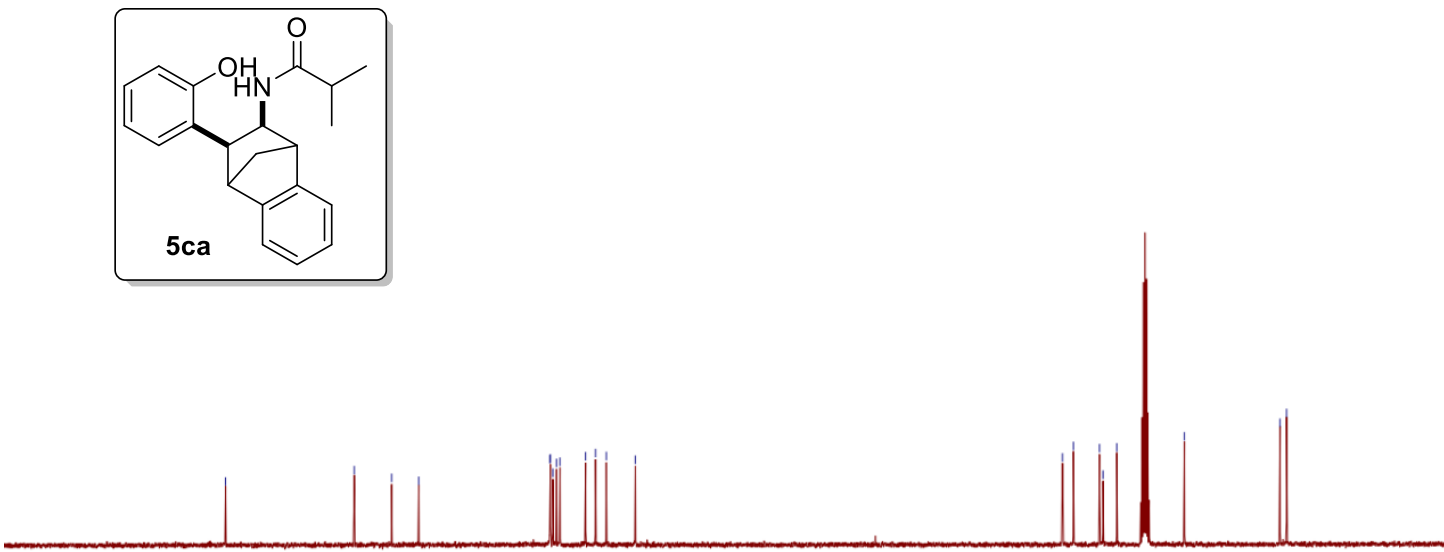

$\begin{array}{lllllllllllllllllllll}200 & 190 & 180 & 170 & 160 & 150 & 140 & 130 & 120 & 110 & 100 & 90 & 80 & 70 & 60 & 50 & 40 & 30 & 20 & 10 & 0\end{array}$ 


\section{${ }^{1} \mathrm{H}$ and ${ }^{13} \mathrm{C}$ NMR of $5 \mathbf{d a}$}

\%.
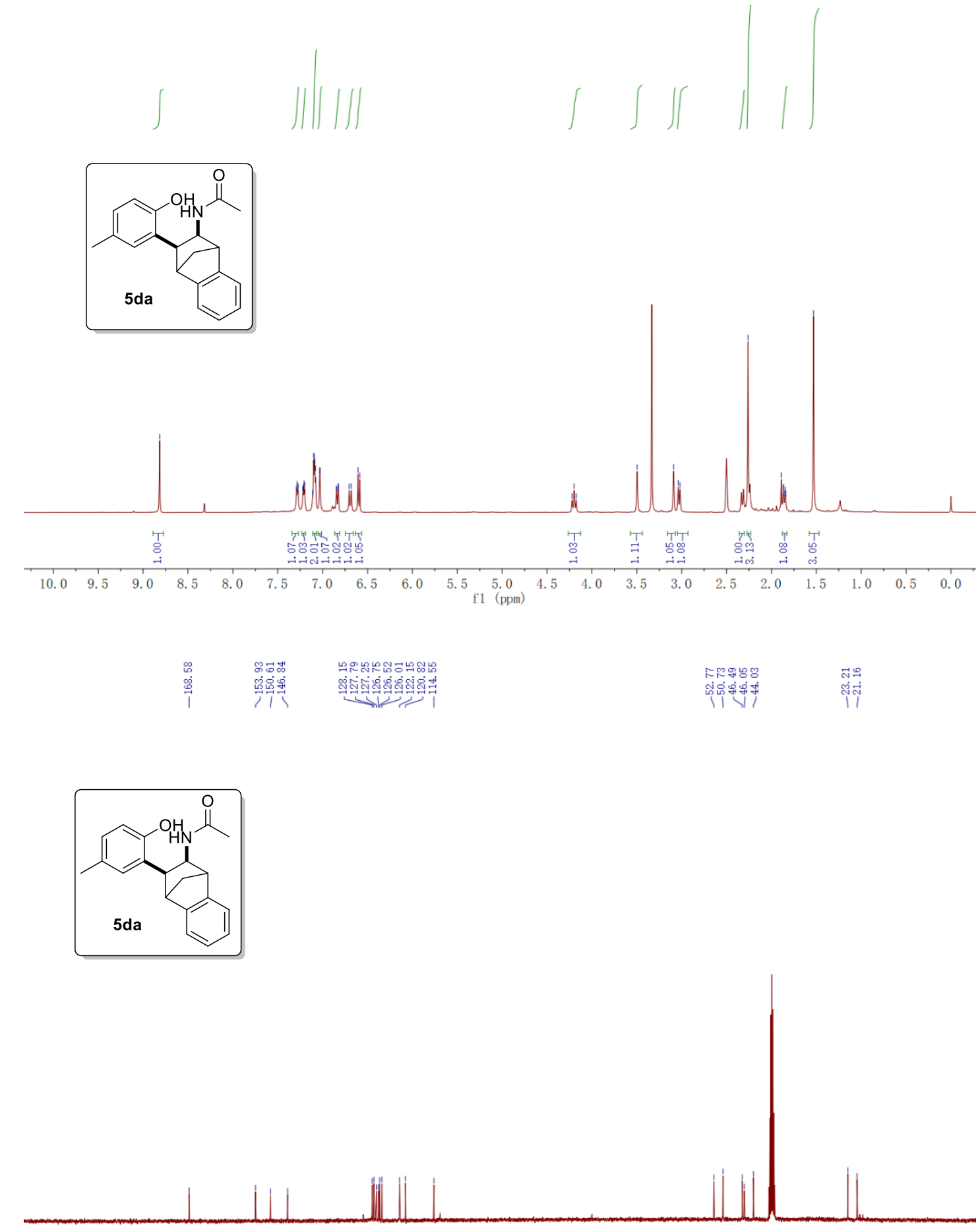

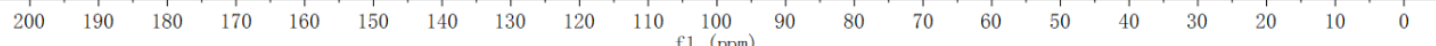


${ }^{1} \mathrm{H}$ and ${ }^{13} \mathrm{C}$ NMR of 5ea

๓ ตำ

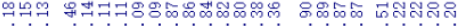

$\underbrace{4 \pi n i n i n i n i n i n g o g o g o s o s}$

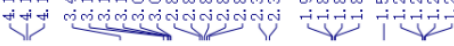

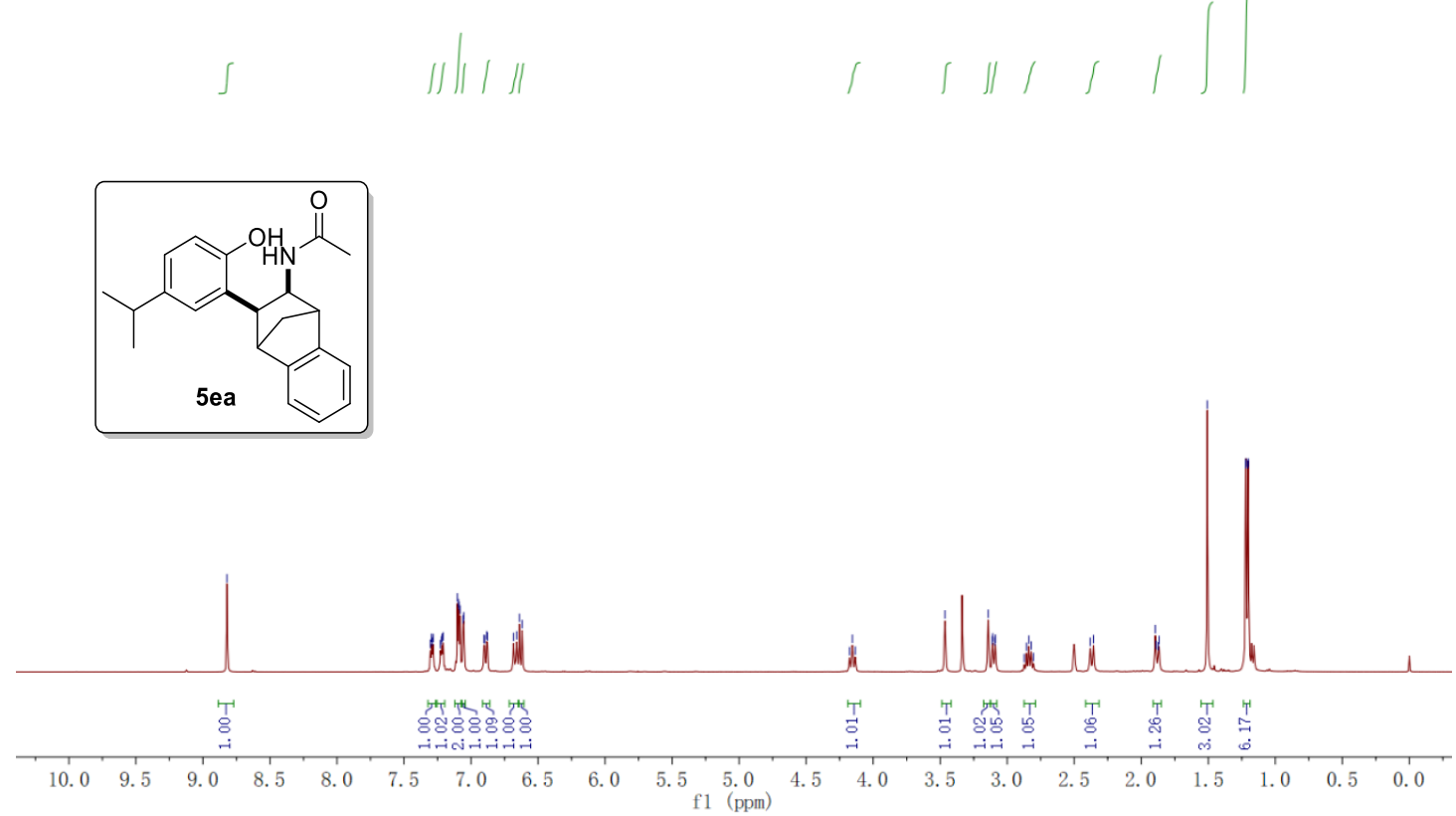

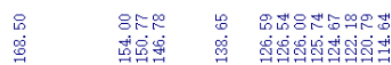

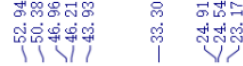
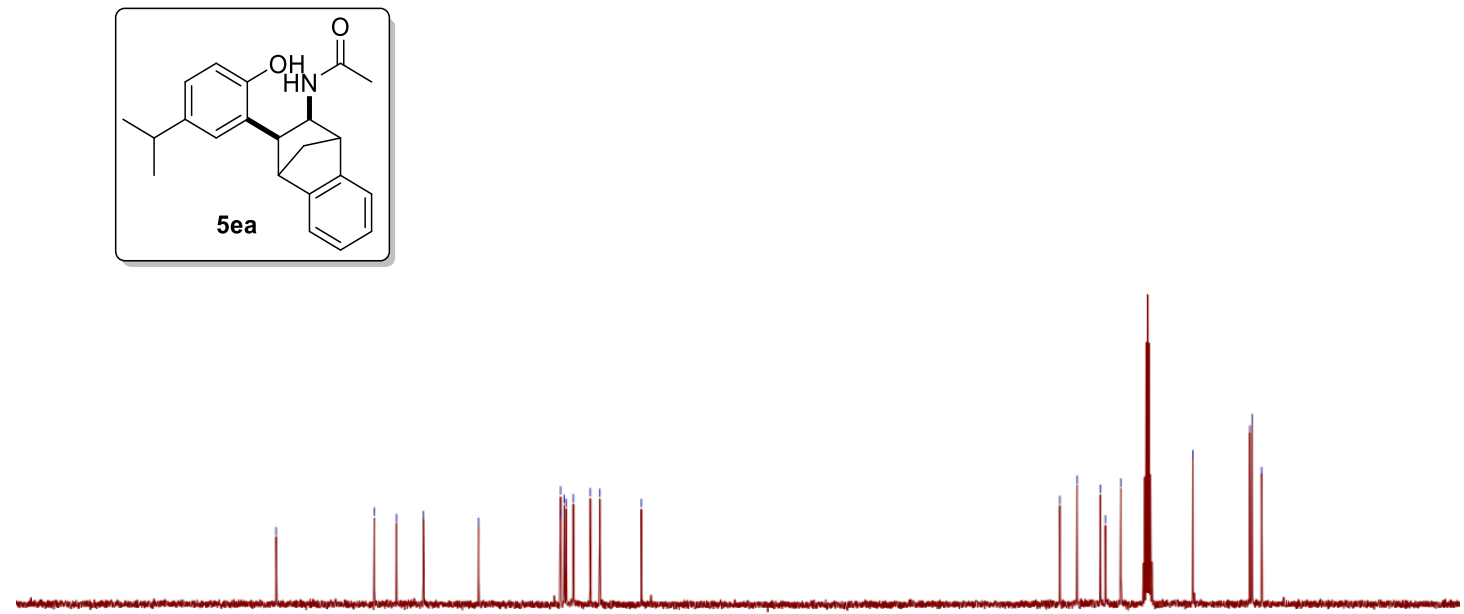

$200 \quad 190$

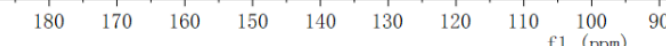
f1 (ppm) 
${ }^{1} \mathrm{H}$ and ${ }^{13} \mathrm{C}$ NMR of $\mathbf{5 f a}$

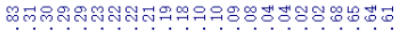

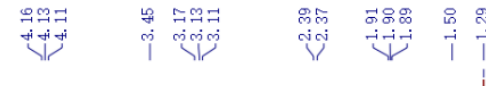

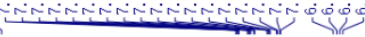

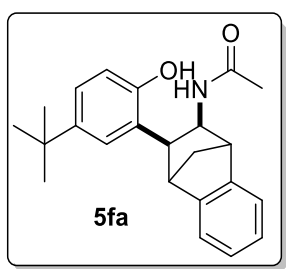

$\iiint_{j} \int$

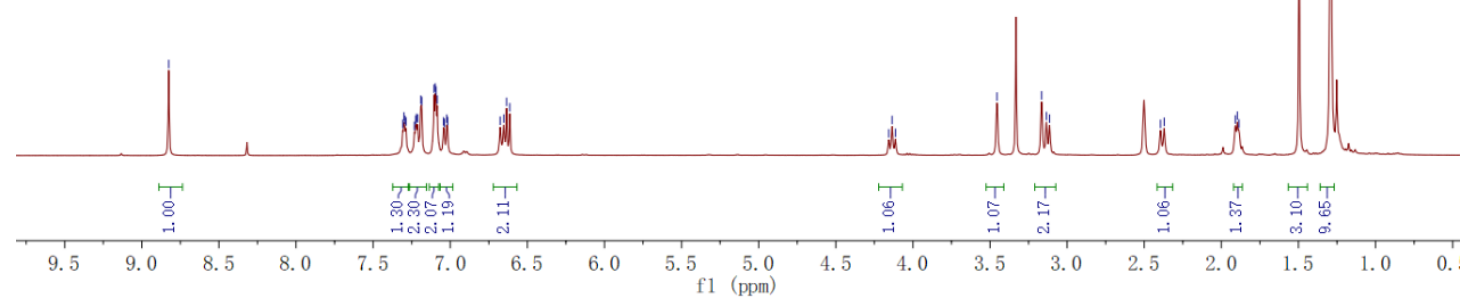

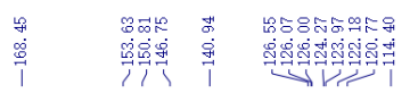

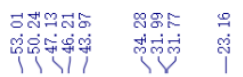

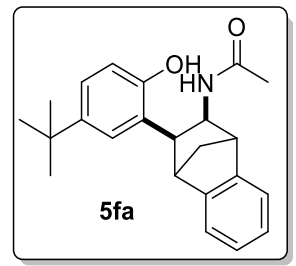

200

$190 \quad 180$

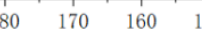

$150 \quad 140 \quad 130$

$120 \quad 1$

$100 \quad 90$

80 


\section{${ }^{1} \mathrm{H}$ and ${ }^{13} \mathrm{C}$ NMR of $\mathbf{5 g a}$}

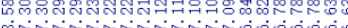

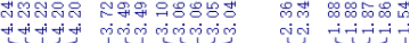

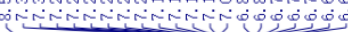

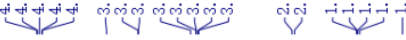

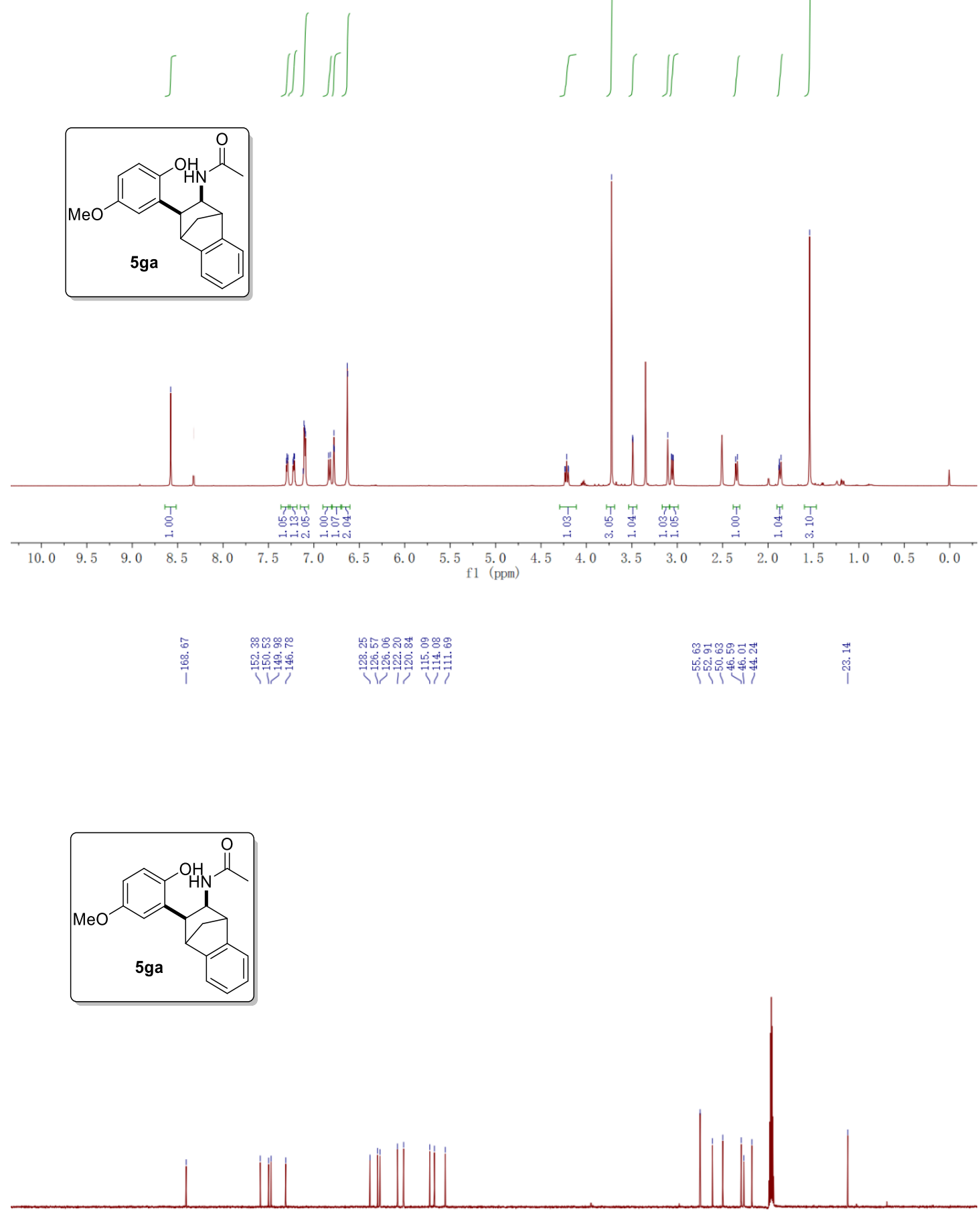

$\begin{array}{lllllllllllllllllllll}200 & 190 & 180 & 170 & 160 & 150 & 140 & 130 & 120 & 110 & 100 & 90 & 80 & 70 & 60 & 50 & 40 & 30 & 20 & 10 & 0\end{array}$ 
${ }^{1} \mathrm{H}{ }^{13} \mathrm{C}$ and ${ }^{19} \mathrm{~F}$ NMR of $\mathbf{5 h a}$

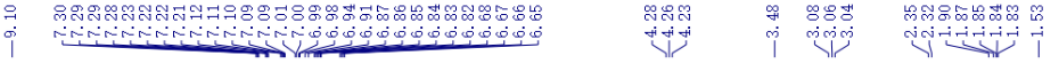
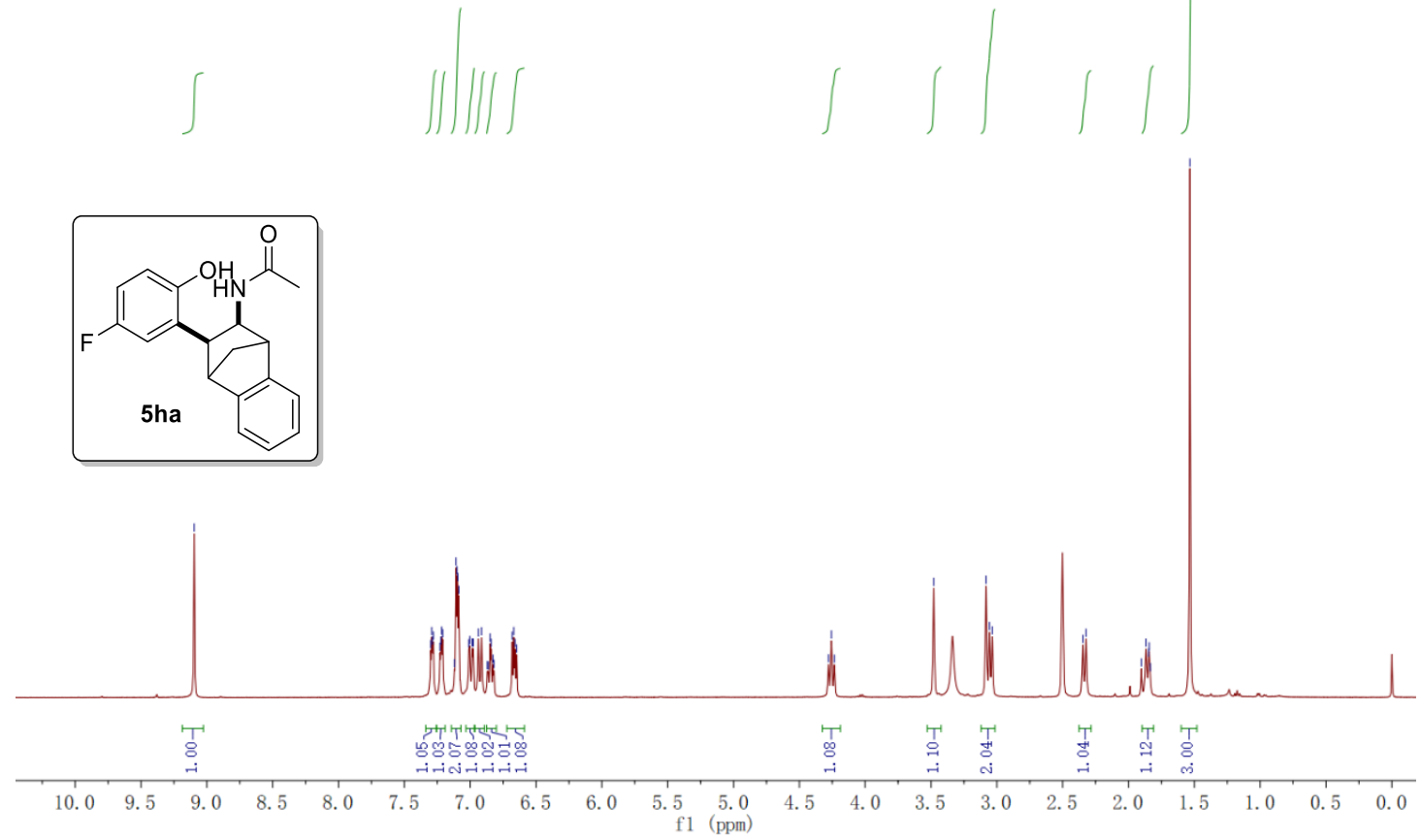

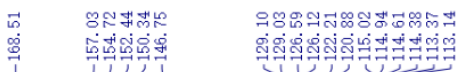

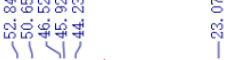
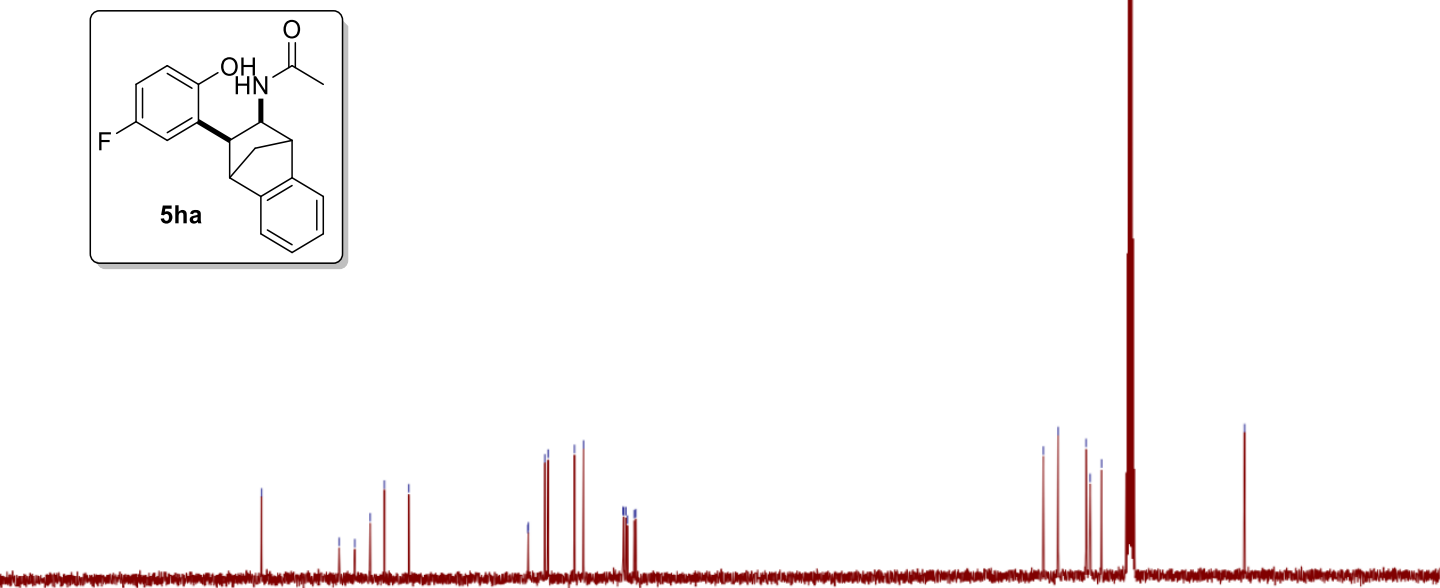

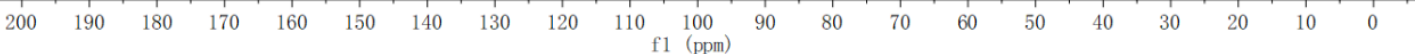



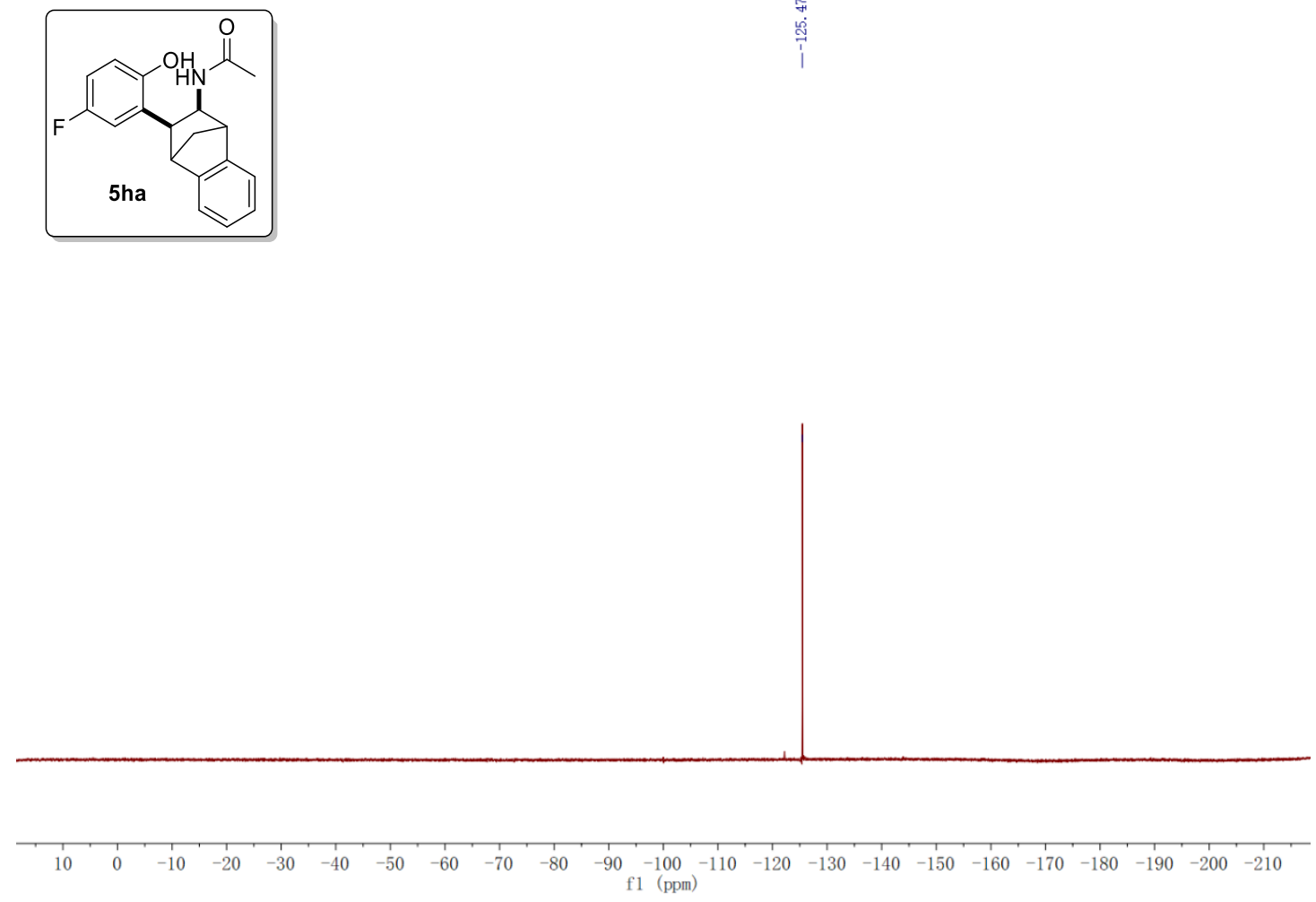


\section{${ }^{1} \mathrm{H}$ and ${ }^{13} \mathrm{C}$ NMR of 5ia}
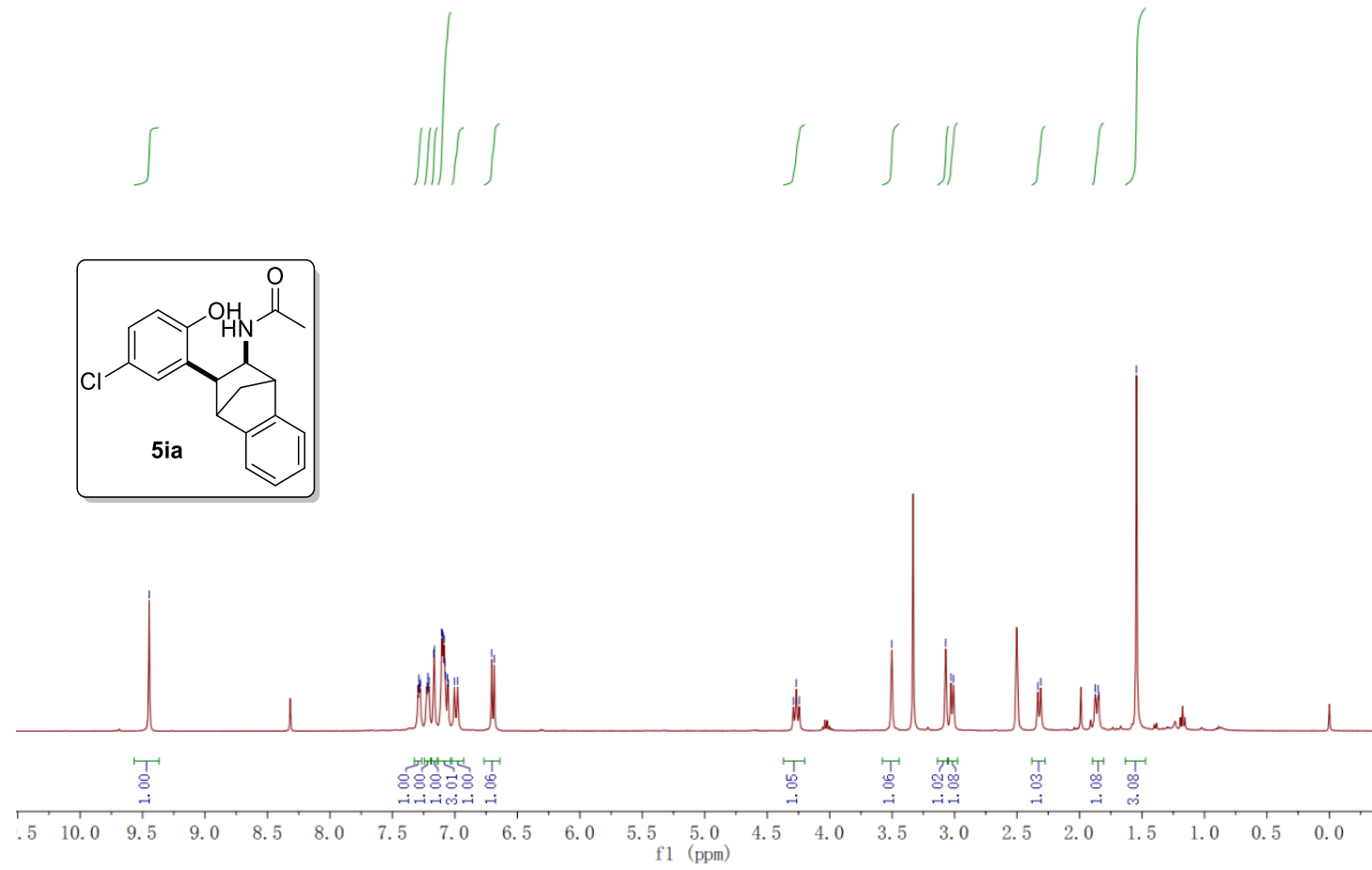

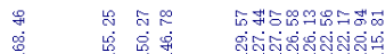
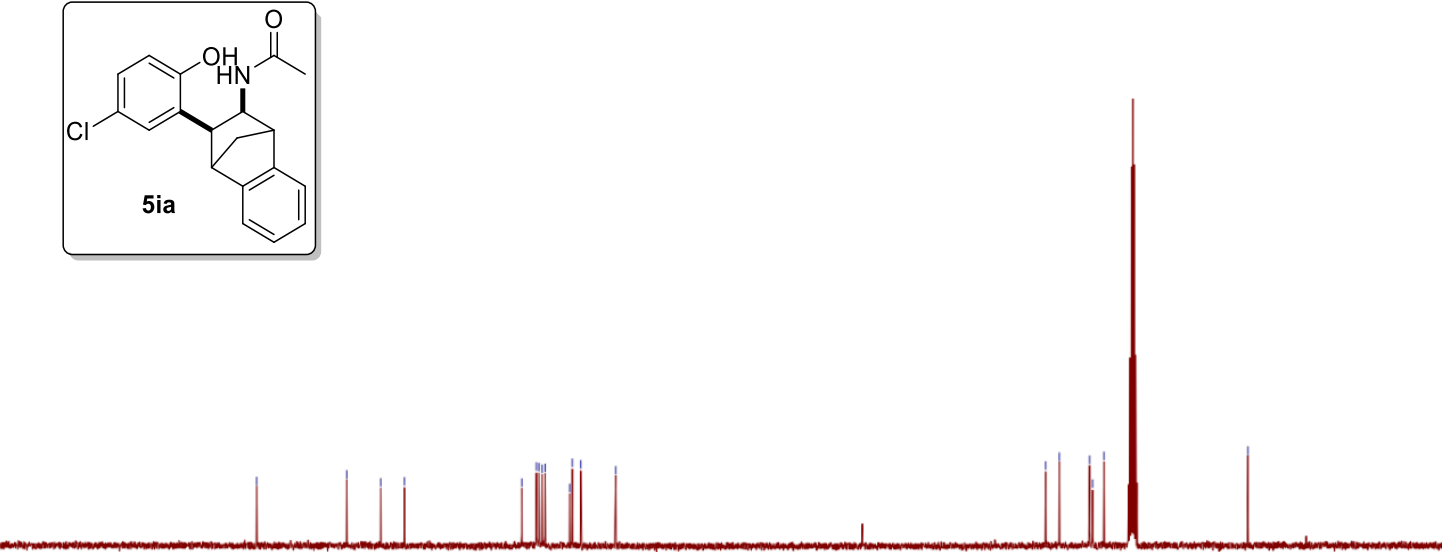

$\begin{array}{llllllllllllllllllllll}200 & 190 & 180 & 170 & 160 & 150 & 140 & 130 & 120 & 110 & 100 & 90 & 80 & 70 & 60 & 50 & 40 & 30 & 20 & 10 & 0\end{array}$ 
${ }^{1} \mathrm{H}$ and ${ }^{13} \mathrm{C}$ NMR of $\mathbf{5 j a}$

\%

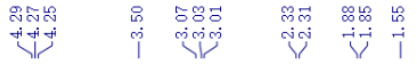
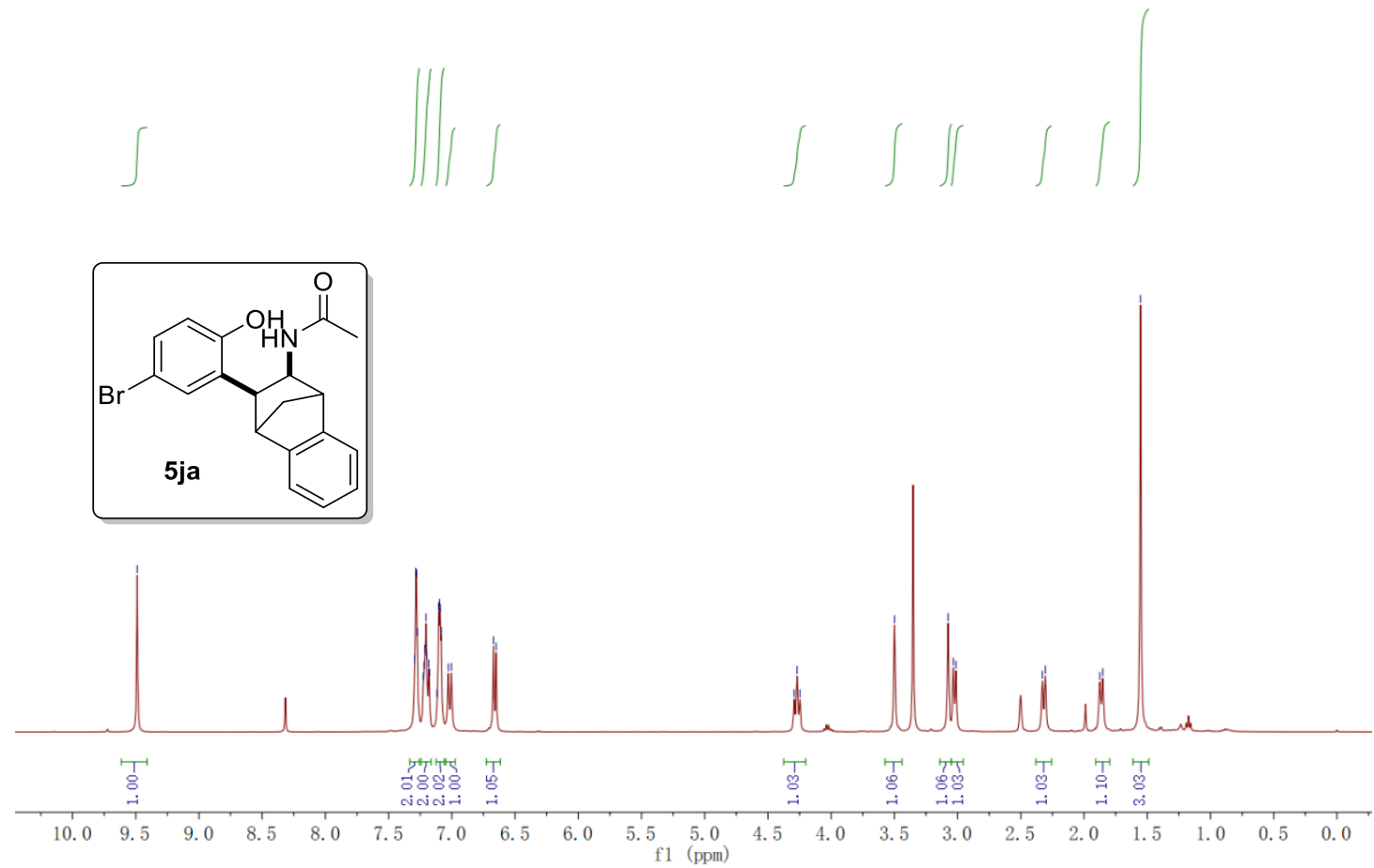

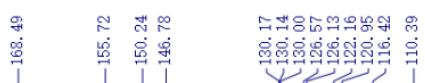

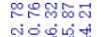

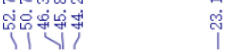
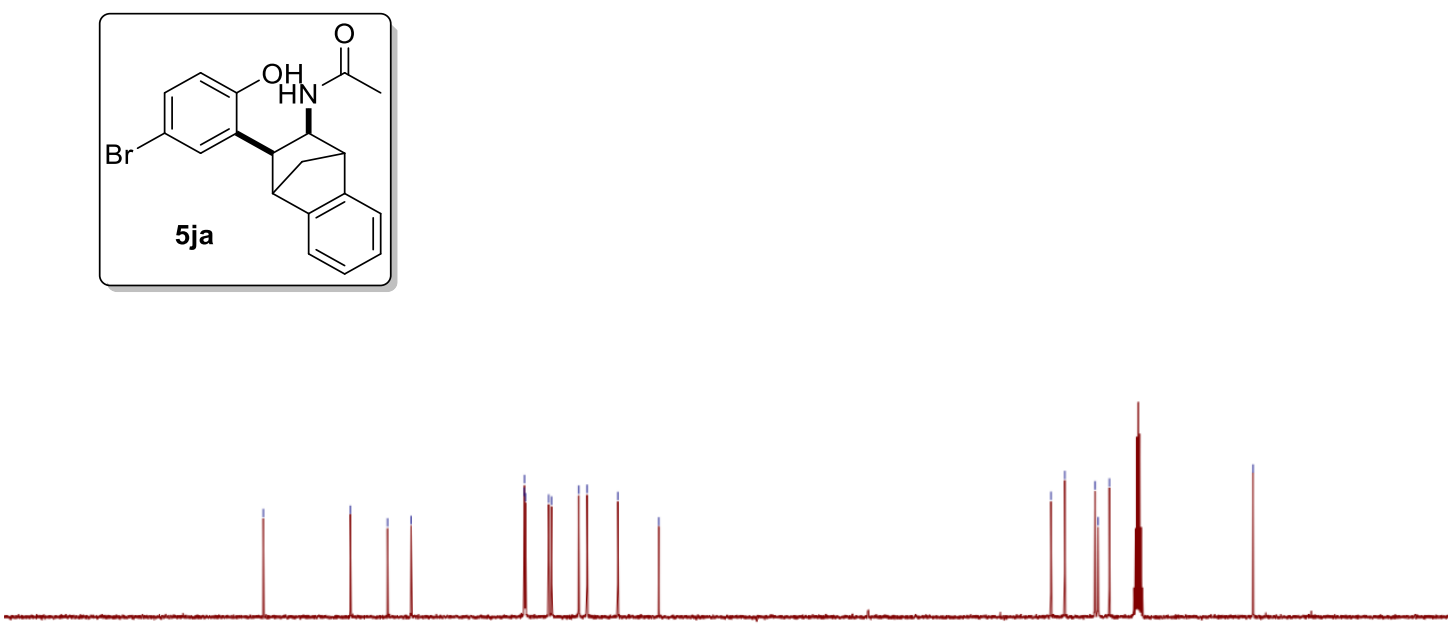

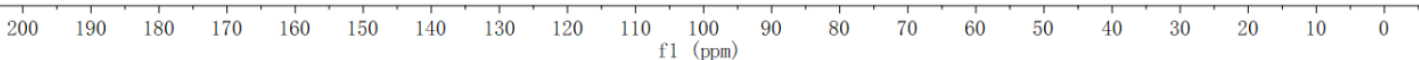




\section{${ }^{1} \mathrm{H}$ and ${ }^{13} \mathrm{C}$ NMR of $\mathbf{5 k a}$}

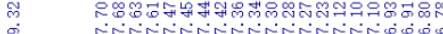

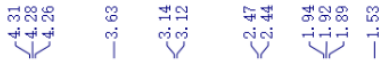
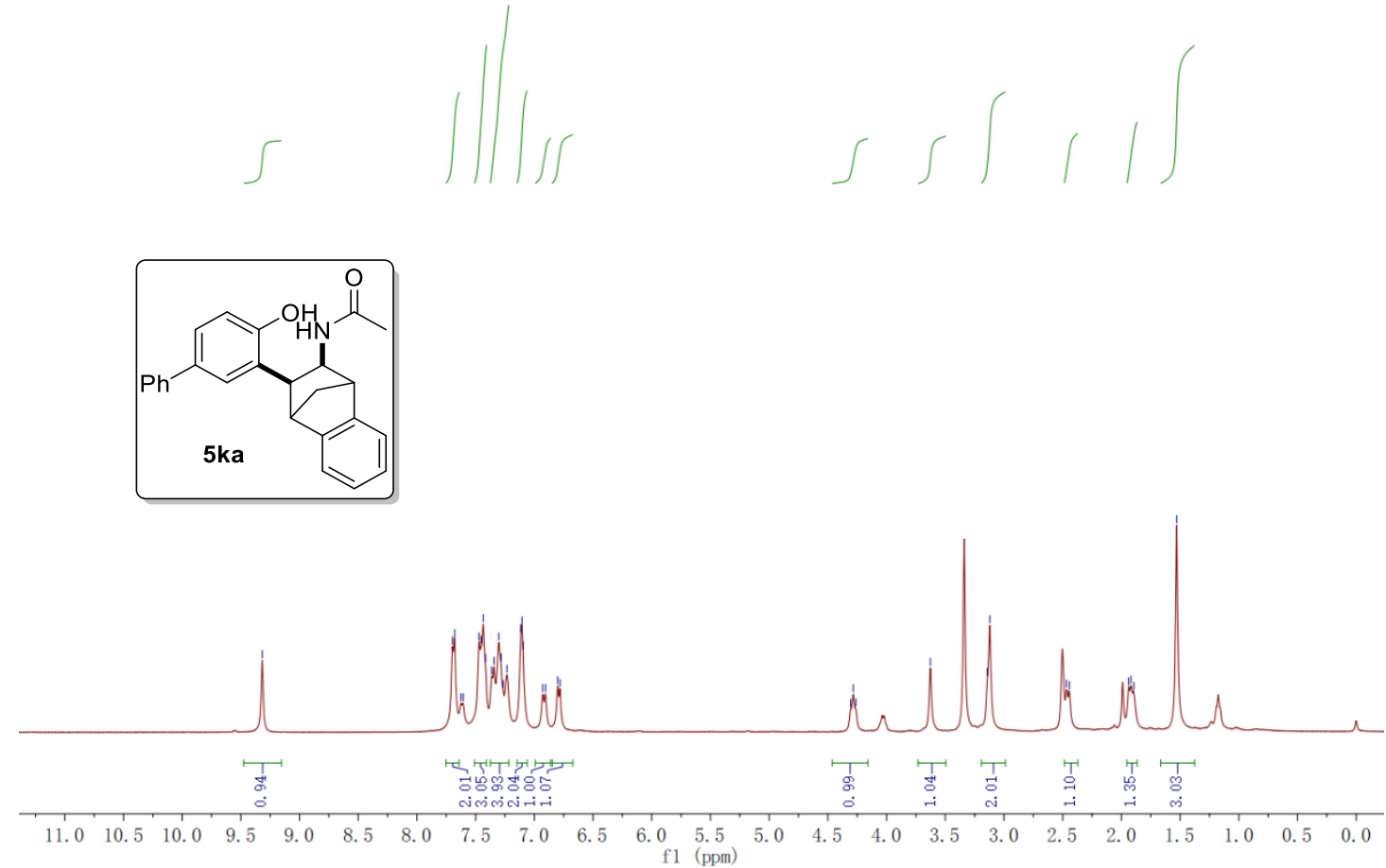

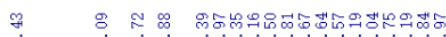

家

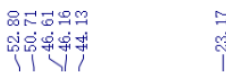
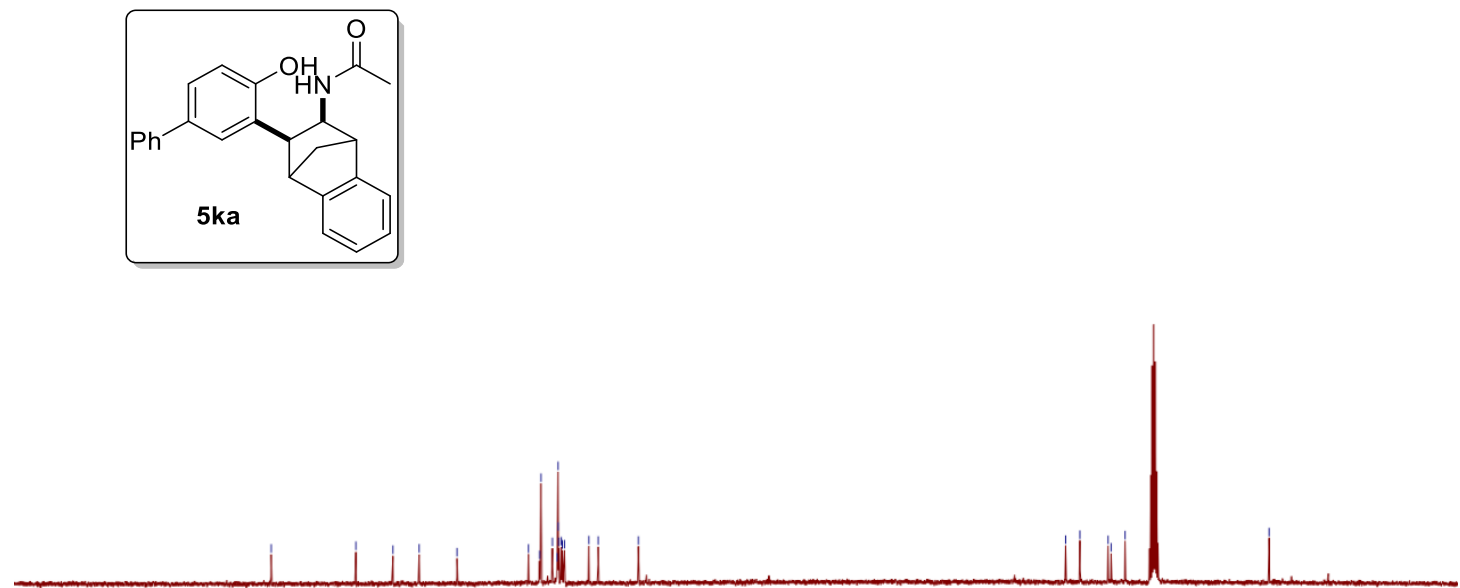

$\begin{array}{lllllllllllllllllllll}200 & 190 & 180 & 170 & 160 & 150 & 140 & 130 & 120 & 110 & 100 & 90 & 80 & 70 & 60 & 50 & 40 & 30 & 20 & 10 & 0\end{array}$ 
${ }^{1} \mathrm{H}$ and ${ }^{13} \mathrm{C}$ NMR of 5 la

$\stackrel{9}{\rightarrow}$

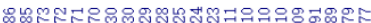

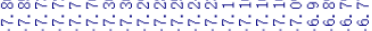

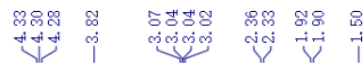

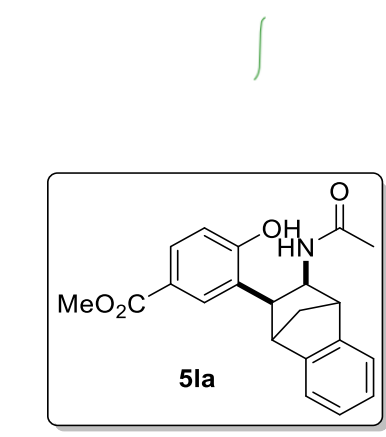

d1 $\| 1$
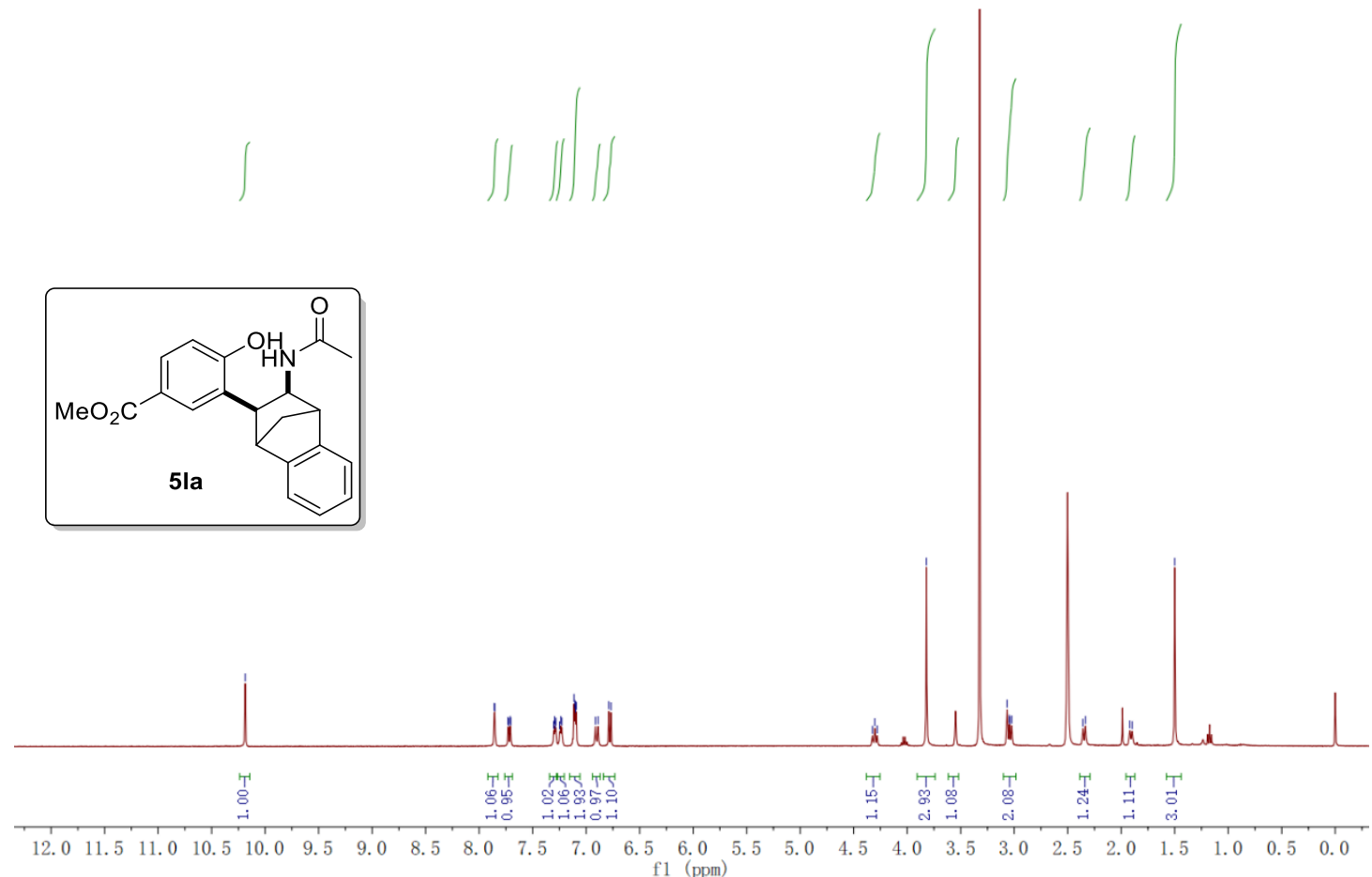

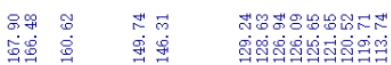

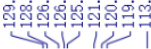

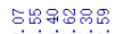

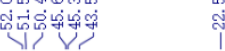
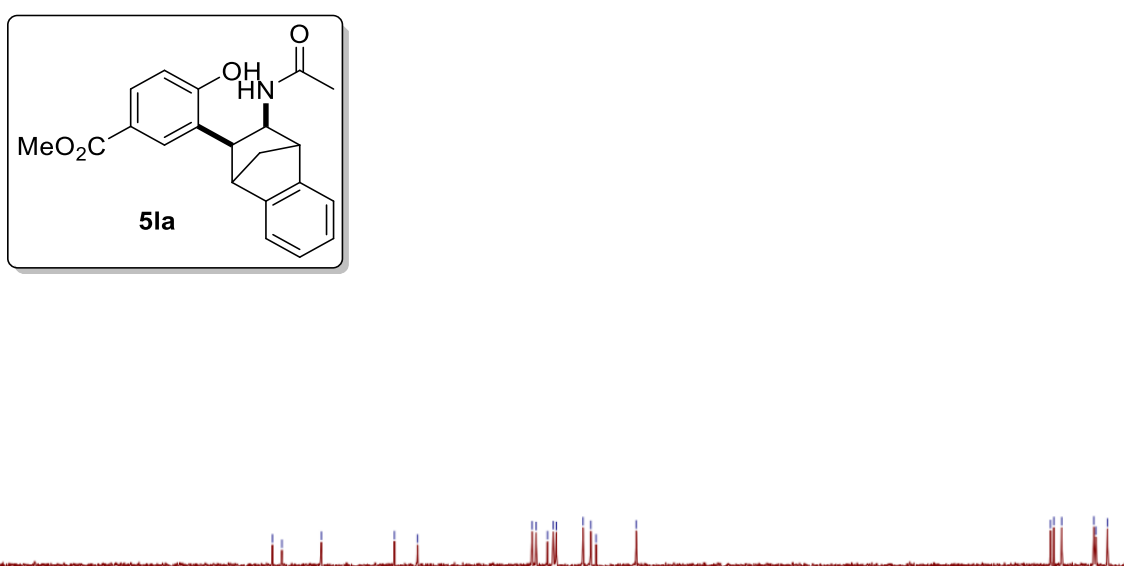

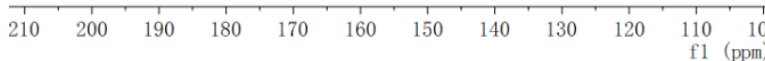


${ }^{1} \mathrm{H}$ and ${ }^{13} \mathrm{C}$ NMR of $\mathbf{5 m a}$

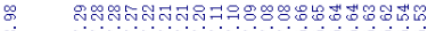

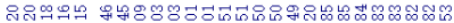

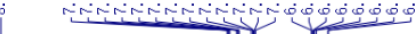

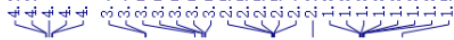
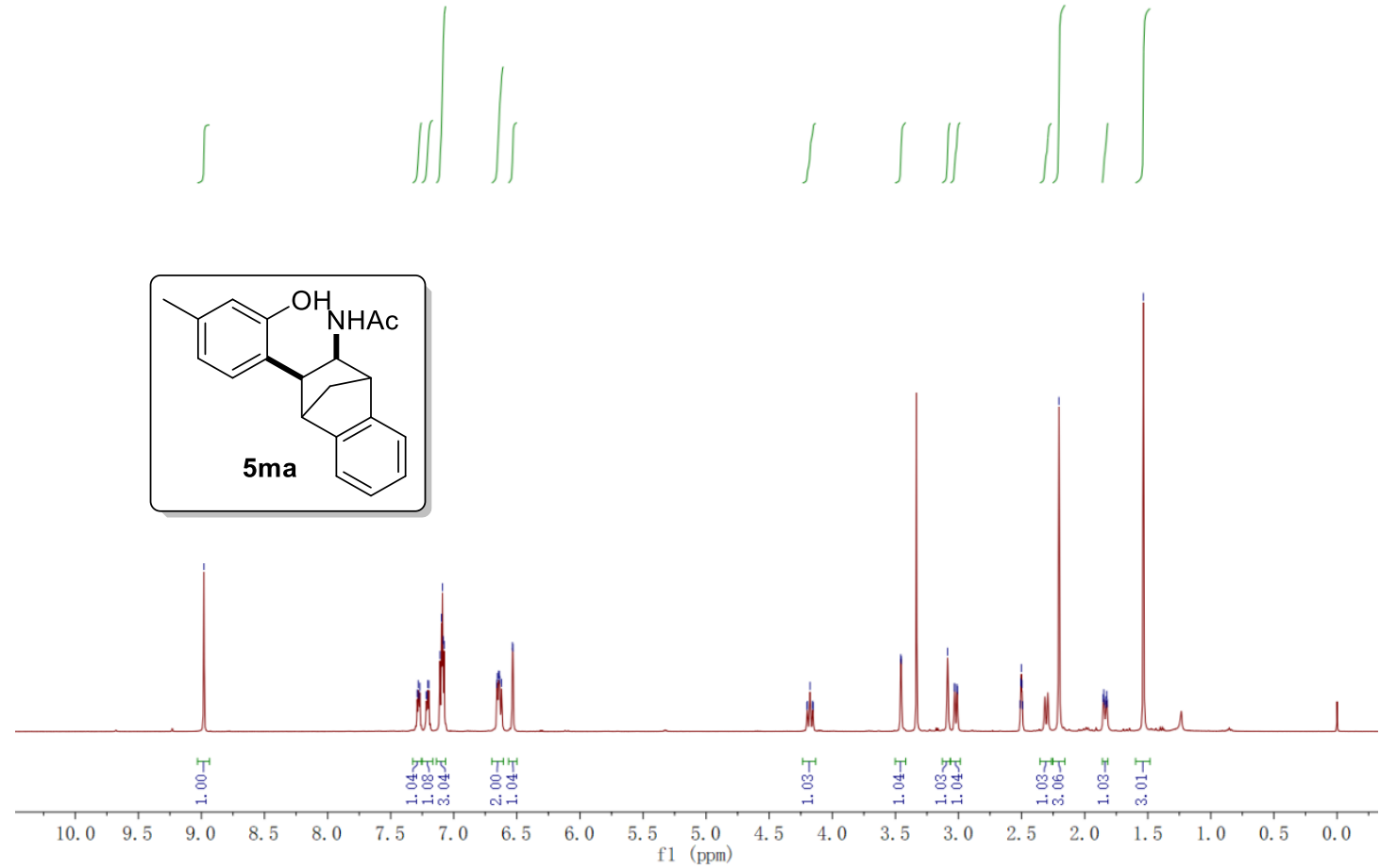

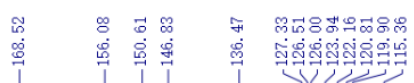

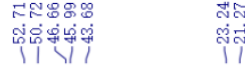
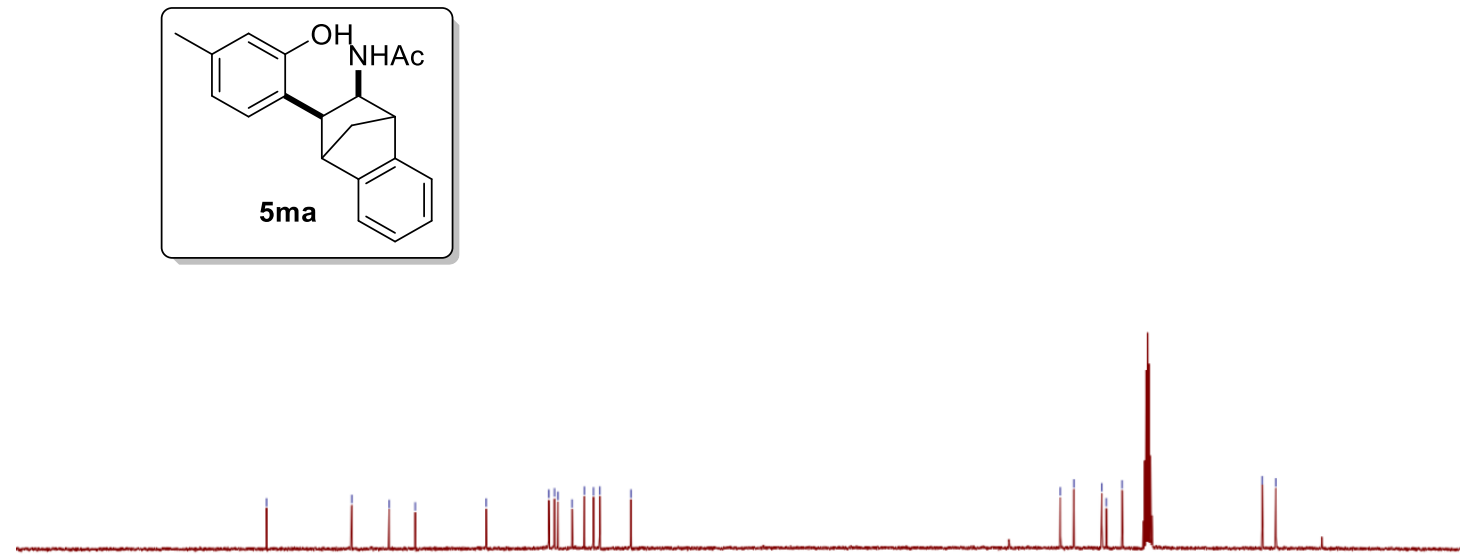

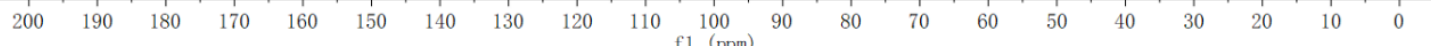


${ }^{1} \mathrm{H}{ }^{13} \mathrm{C}$ and ${ }^{19} \mathrm{~F}$ NMR of $\mathbf{5 n a}$

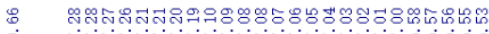

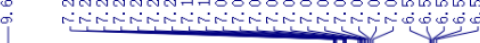

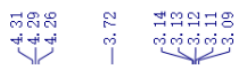

$\int$
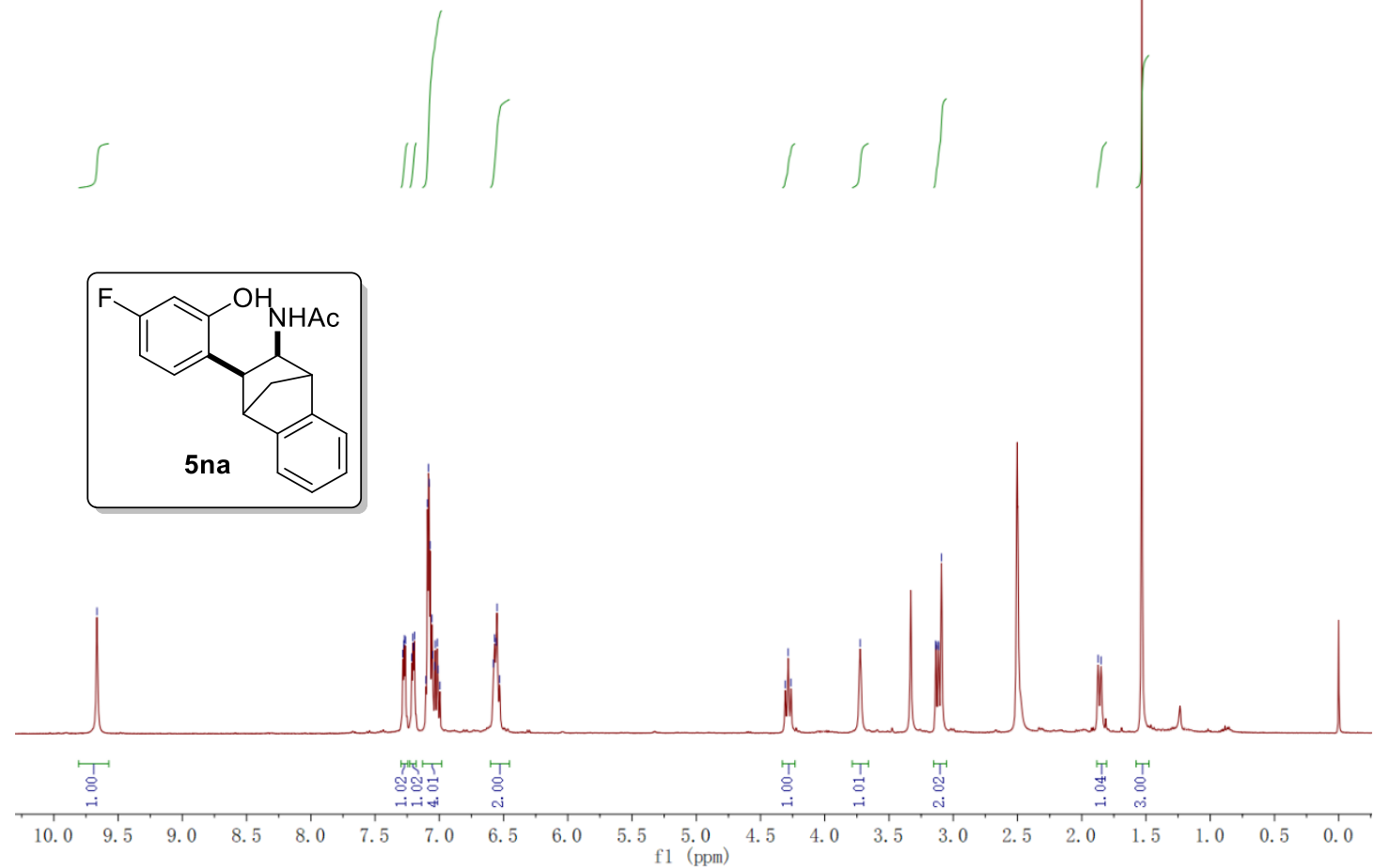

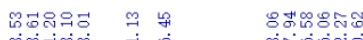

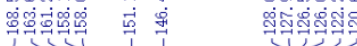

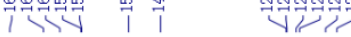

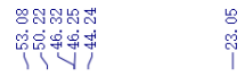
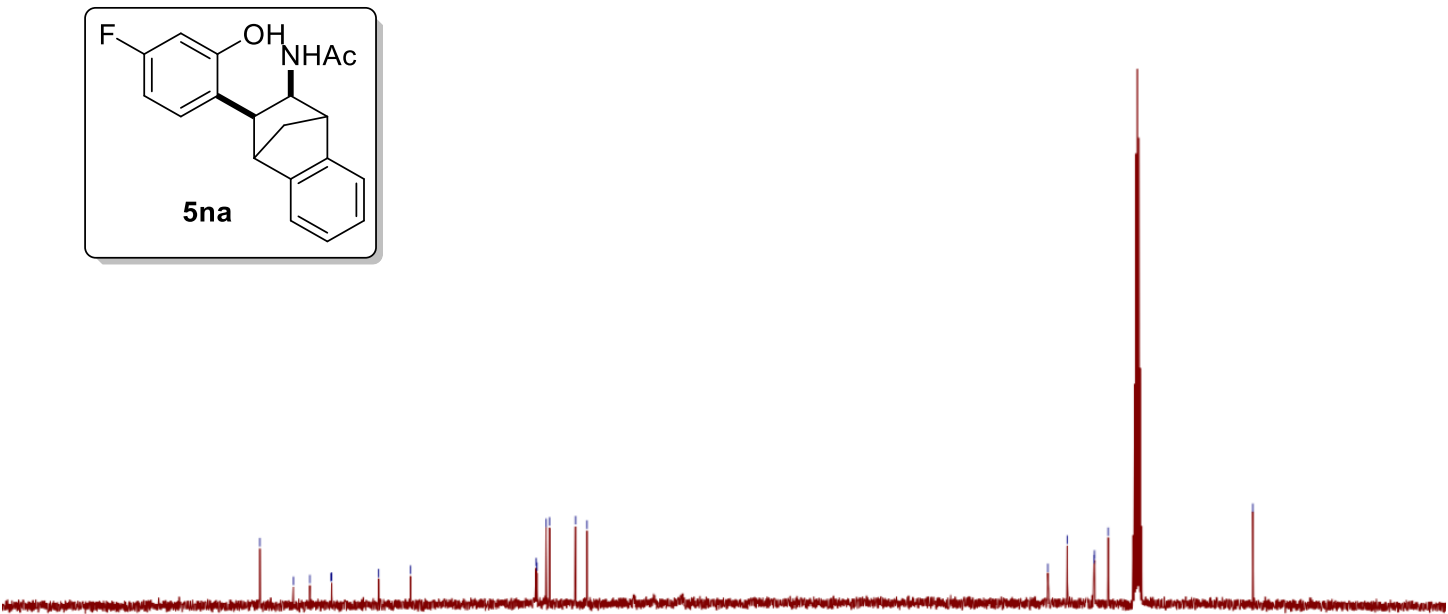

$\begin{array}{lllllllllllllllllllll}200 & 190 & 180 & 170 & 160 & 150 & 140 & 130 & 120 & 110 & 100 & 90 & 80 & 70 & 60 & 50 & 40 & 30 & 20 & 10 & 0\end{array}$ 

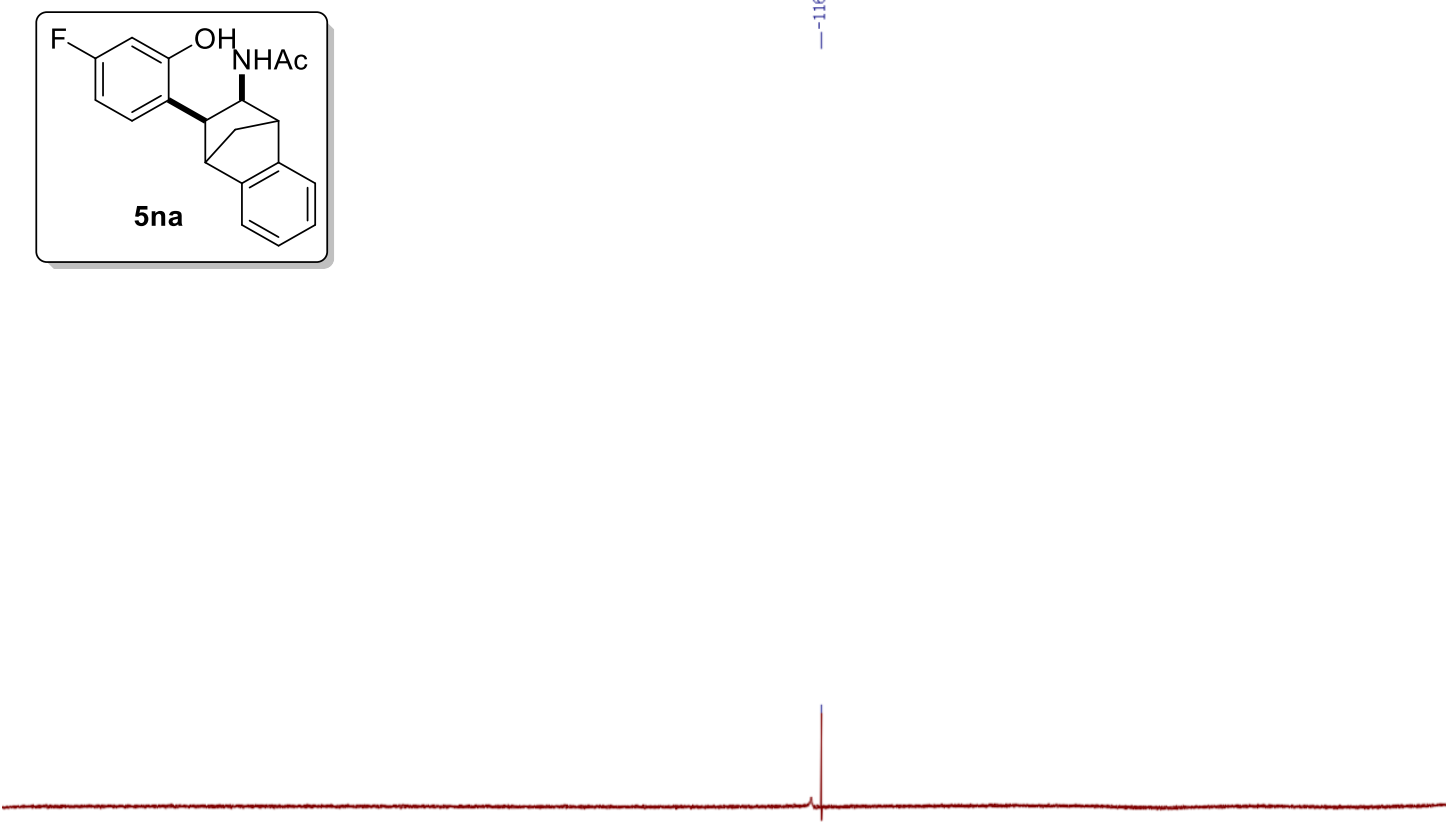

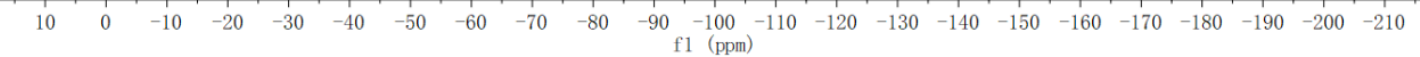


${ }^{1} \mathrm{H}$ and ${ }^{13} \mathrm{C}$ NMR of $\mathbf{5 0 a}$
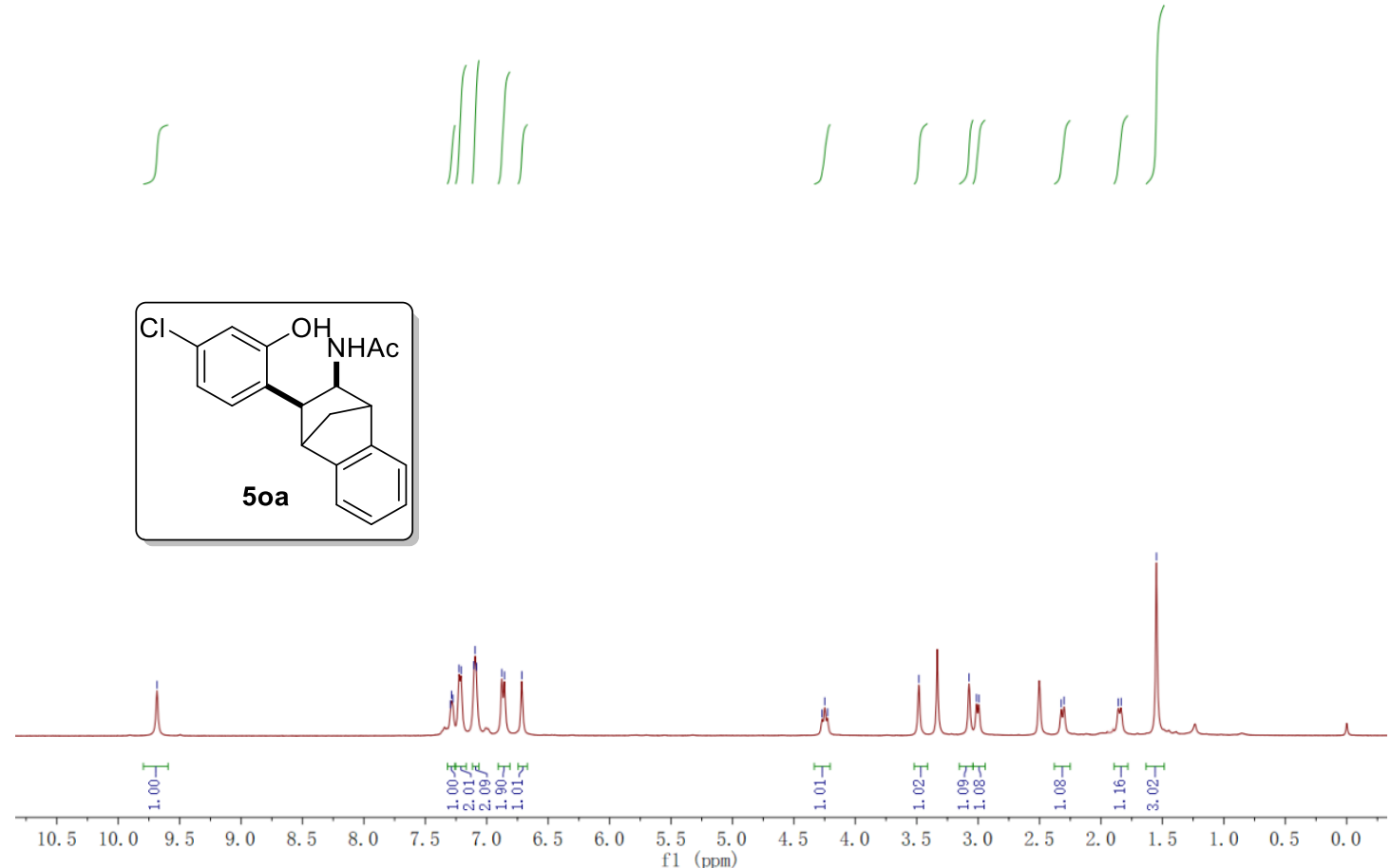

\section{๘

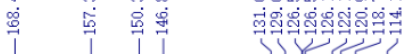

f1 (ppm)

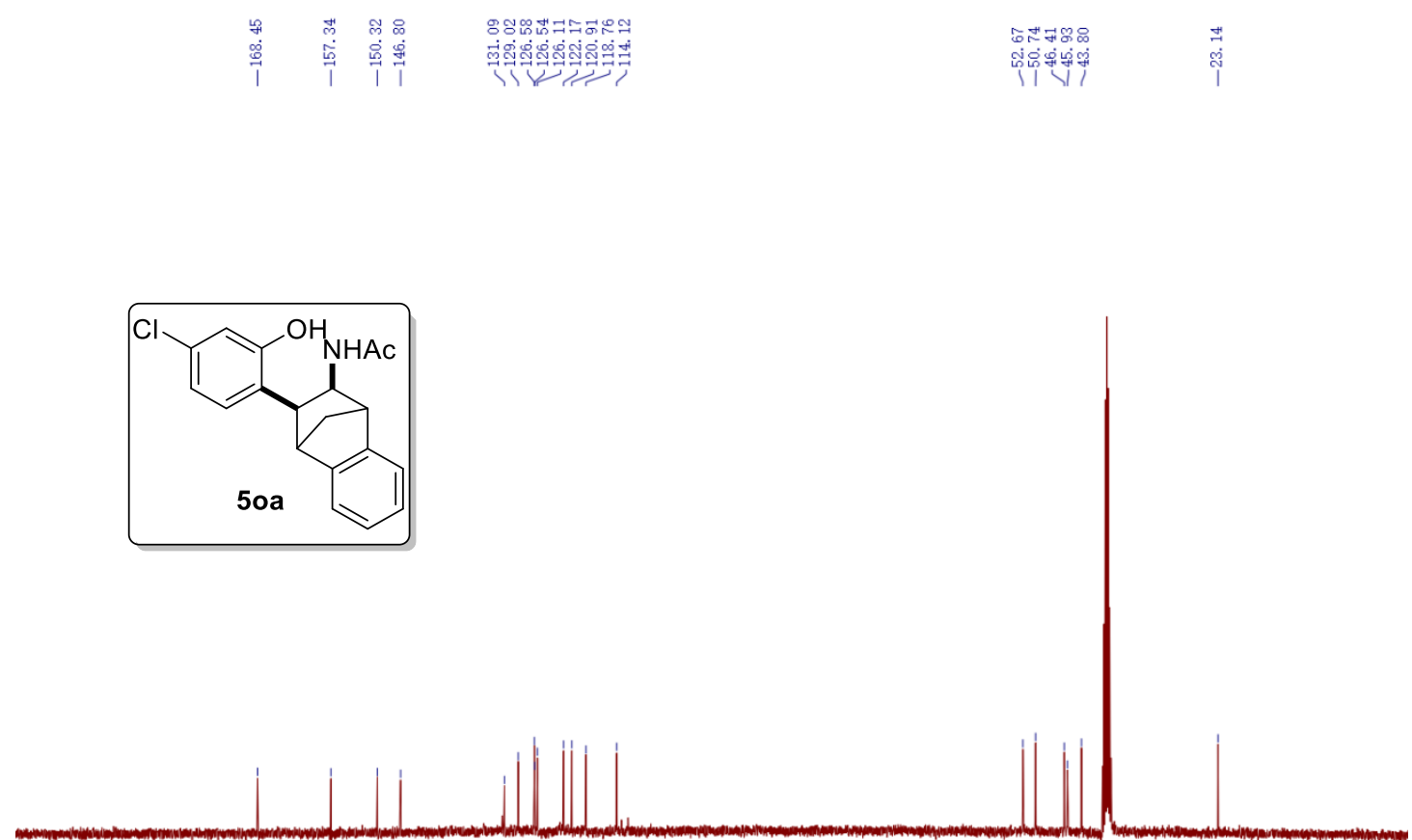

$\begin{array}{llllllllllllllllllllllll}200 & 190 & 180 & 170 & 160 & 150 & 140 & 130 & 120 & 110 & 100 & 90 & 80 & 70 & 60 & 50 & 40 & 30 & 20 & 10 & 0\end{array}$ 


\section{${ }^{1} \mathrm{H}$ and ${ }^{13} \mathrm{C}$ NMR of 5 pa}

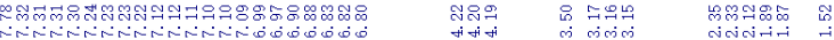

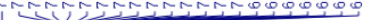
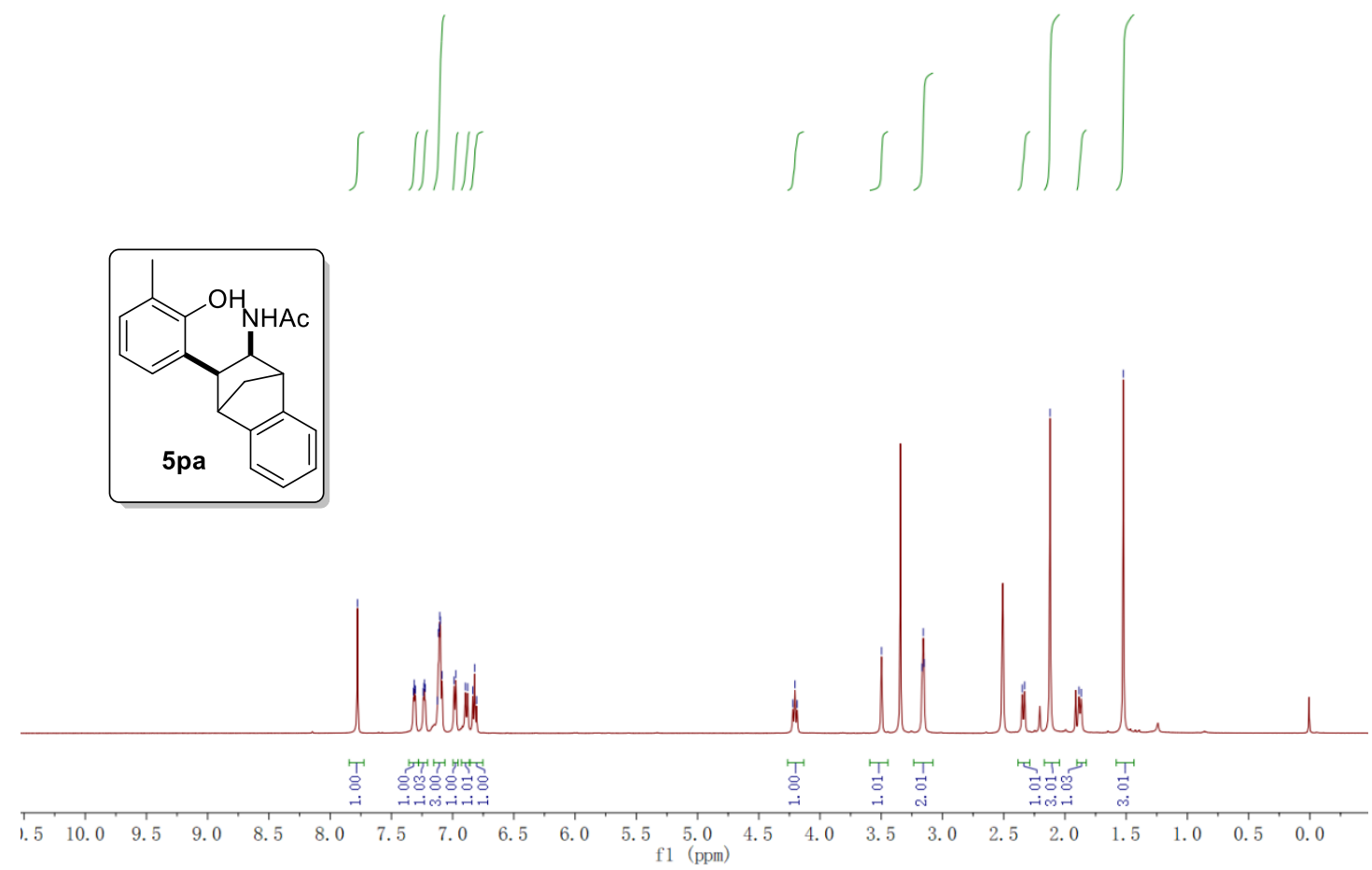

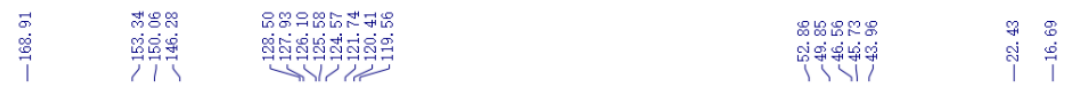
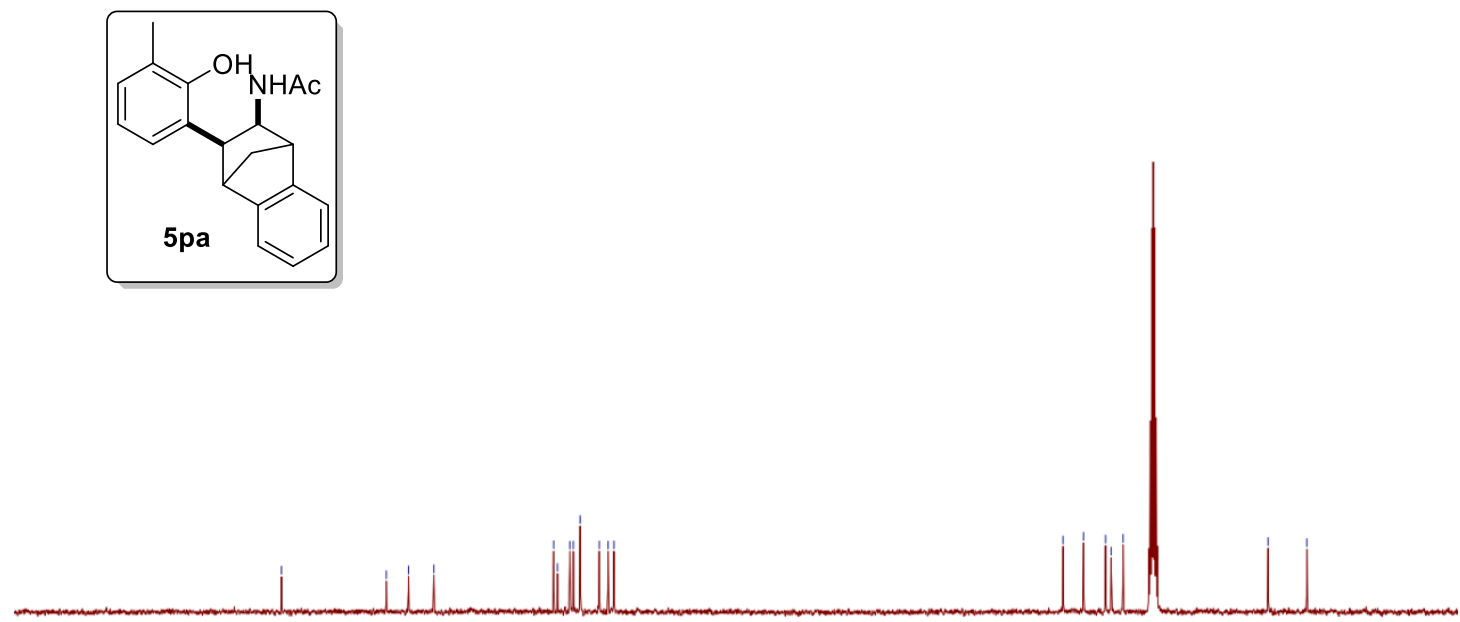

$\begin{array}{lllllllllllllllllllll}200 & 190 & 180 & 170 & 160 & 150 & 140 & 130 & 120 & 110 & 100 & 90 & 80 & 70 & 60 & 50 & 40 & 30 & 20 & 10 & 0\end{array}$ 
${ }^{1} \mathrm{H}$ and ${ }^{13} \mathrm{C}$ NMR of $\mathbf{5 q a}$

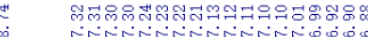

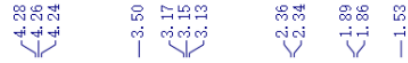
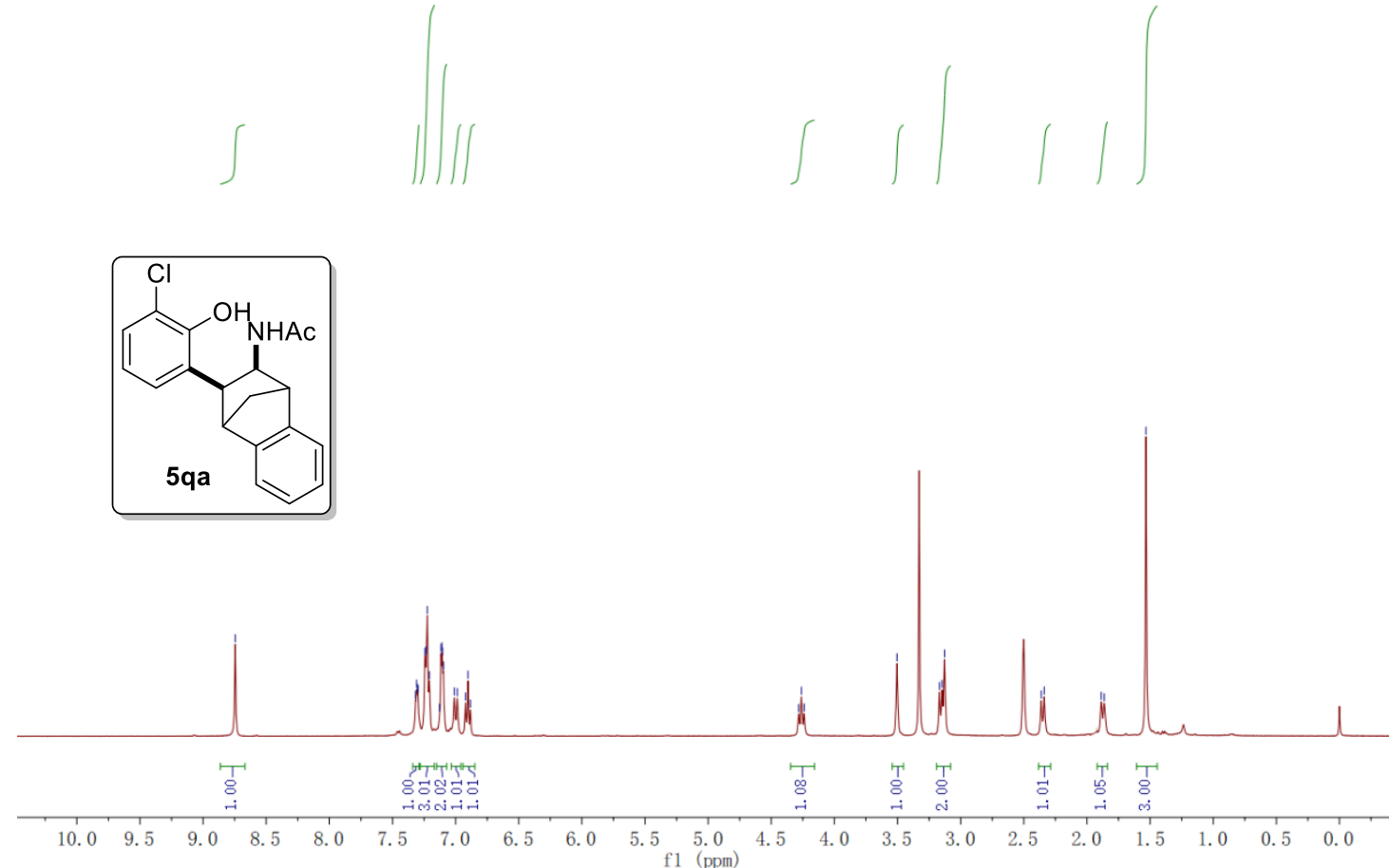

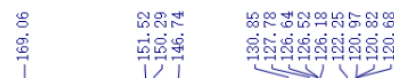

도유ำ

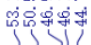
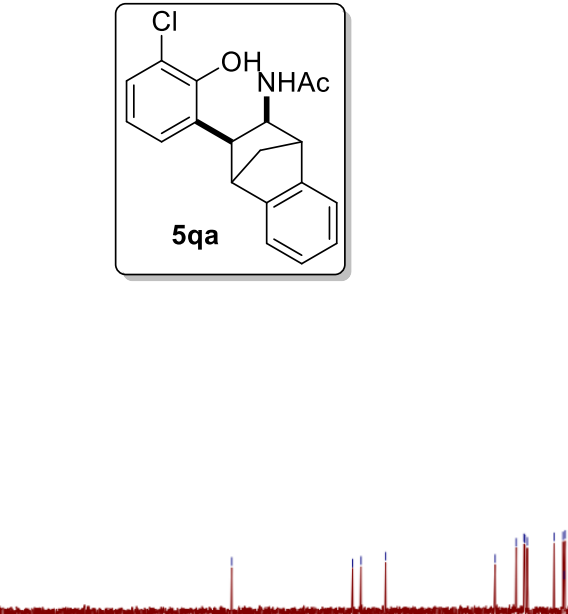

$200 \quad 190$

$180 \quad 170$

$160 \quad 150$

$10 \quad 100 \quad 90$

$\begin{array}{lll}80 & 60\end{array}$

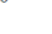

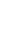




\section{${ }^{1} \mathrm{H}$ and ${ }^{13} \mathrm{C}$ NMR of 5 ra}

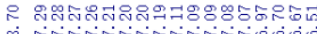

o inisinininiviniogos

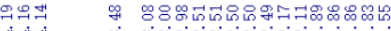

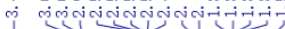
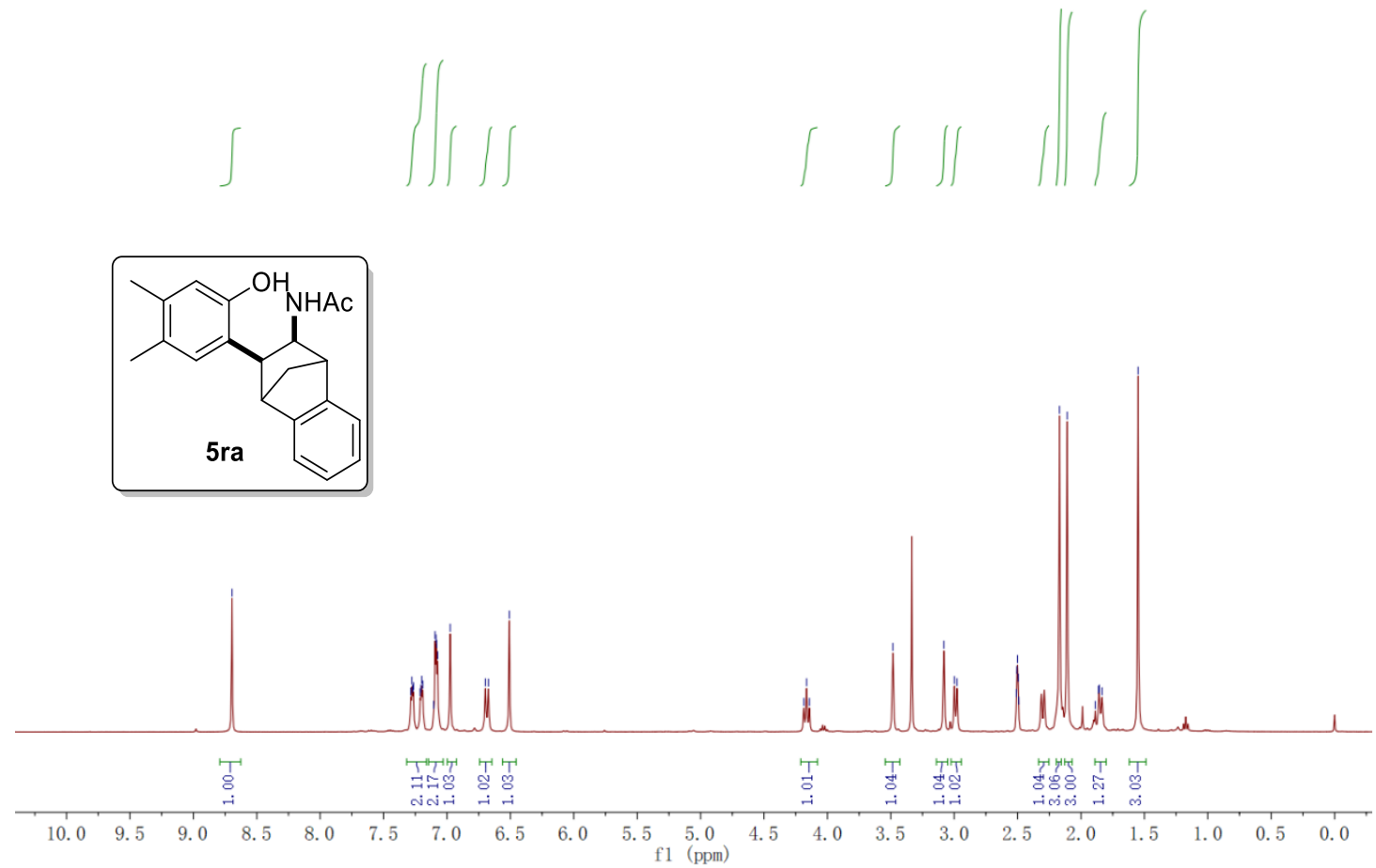

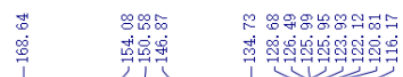

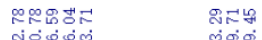

ำ

พู่ฐำ
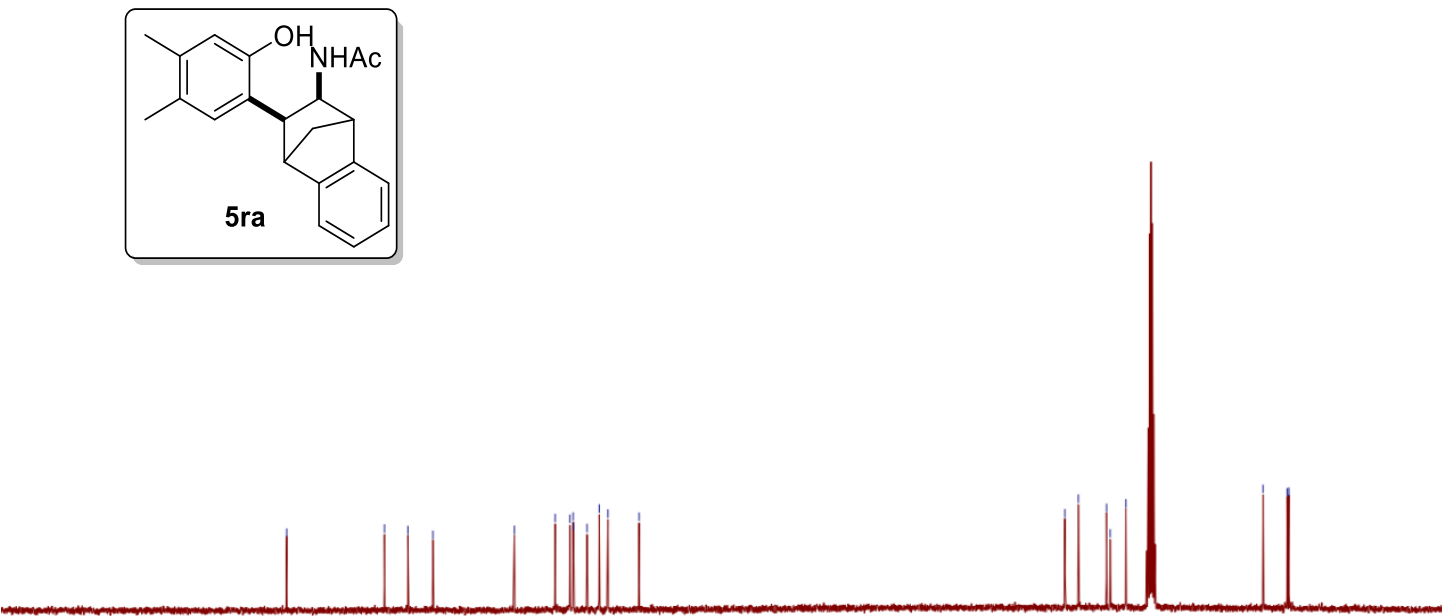

$\begin{array}{llllllllllllllllllllllllll}10 & 200 & 190 & 180 & 170 & 160 & 150 & 140 & 130 & 120 & 110 & 100 & 90 & 80 & 70 & 60 & 50 & 40 & 30 & 20 & 10 & 0\end{array}$ 
${ }^{1} \mathrm{H}^{13} \mathrm{C}$ and ${ }^{19} \mathrm{~F}$ NMR of $\mathbf{5 s a}$

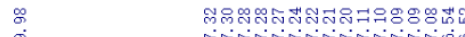

inivinitininivivitiog

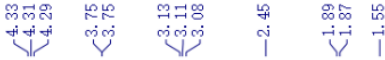
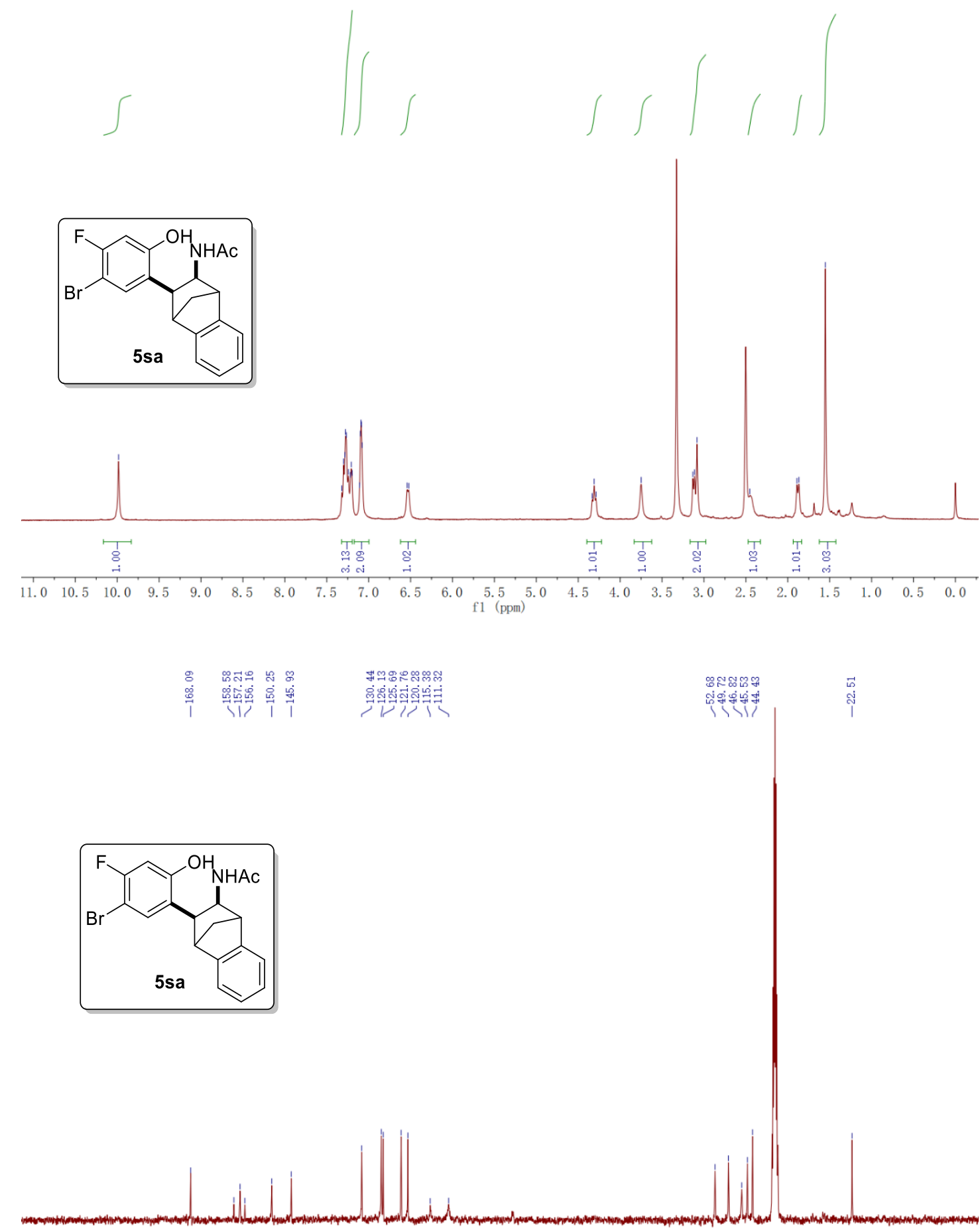

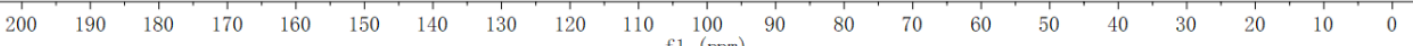




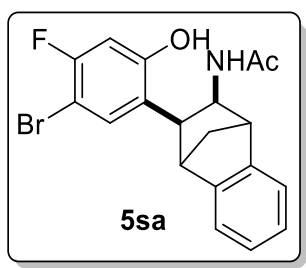

$\stackrel{8}{0}$

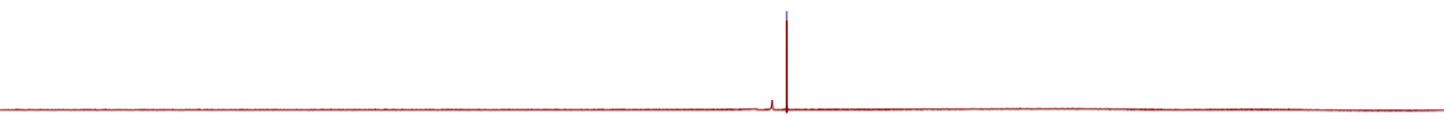

$\begin{array}{lllllllllllllllllllllllll}1 & 0 & 0 & -10 & -20 & -30 & -40 & -50 & -60 & -70 & -80 & -90 & -100 & -110 & -120 & -130 & -140 & -150 & -160 & -170 & -180 & -190 & -200 & -210\end{array}$ 
${ }^{1} \mathrm{H}$ and ${ }^{13} \mathrm{C}$ NMR of 5 ta
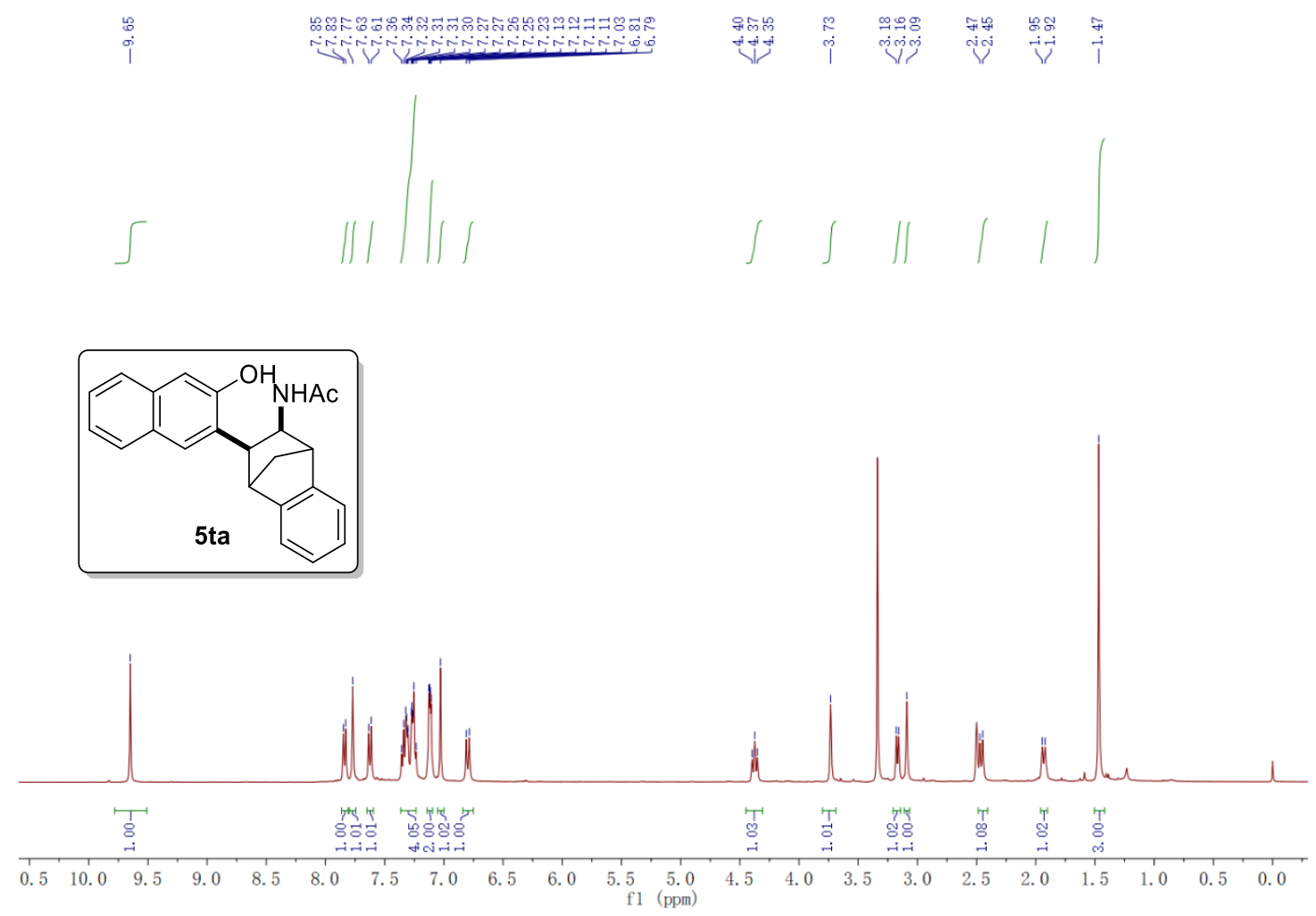

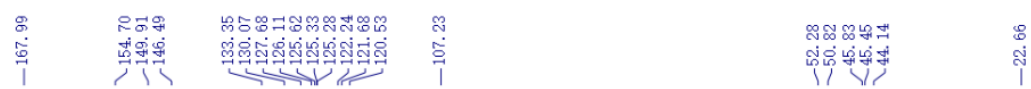
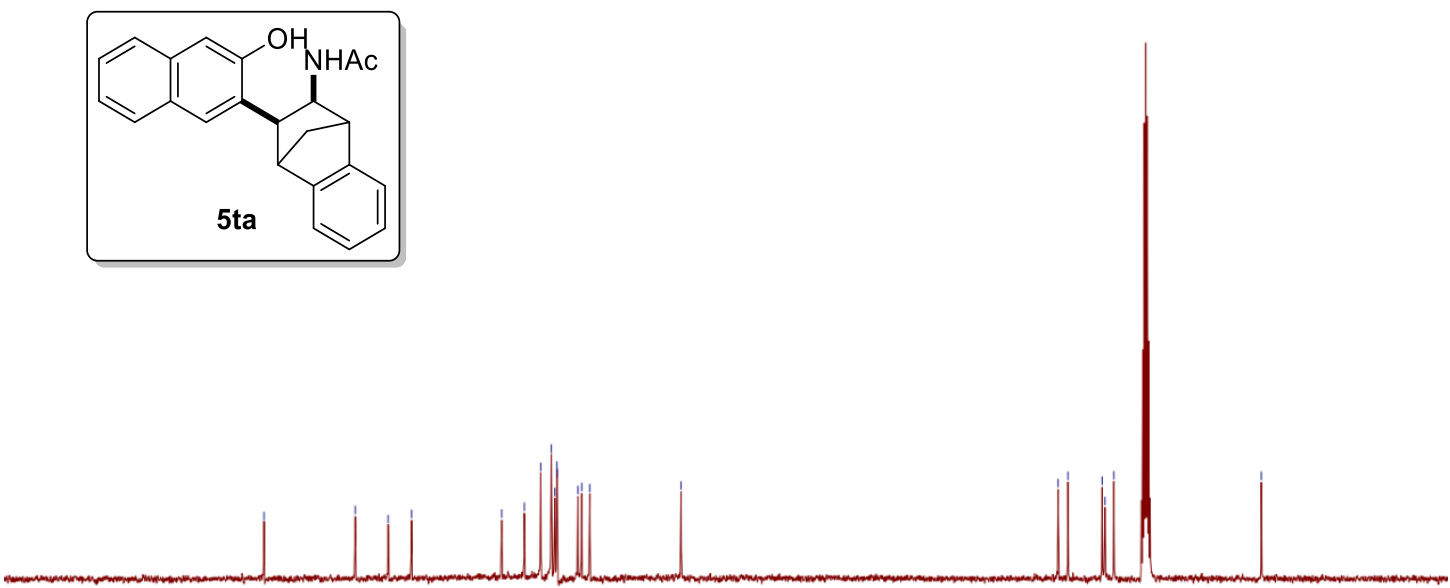

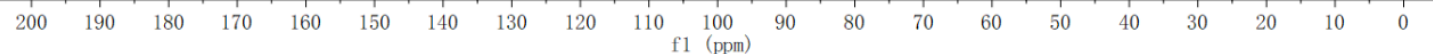


${ }^{1} \mathrm{H}$ and ${ }^{13} \mathrm{C}$ NMR of 5 ua

$\vec{\sigma} \quad$ 이ำ

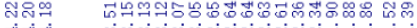

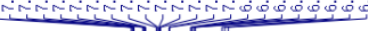

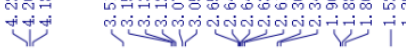

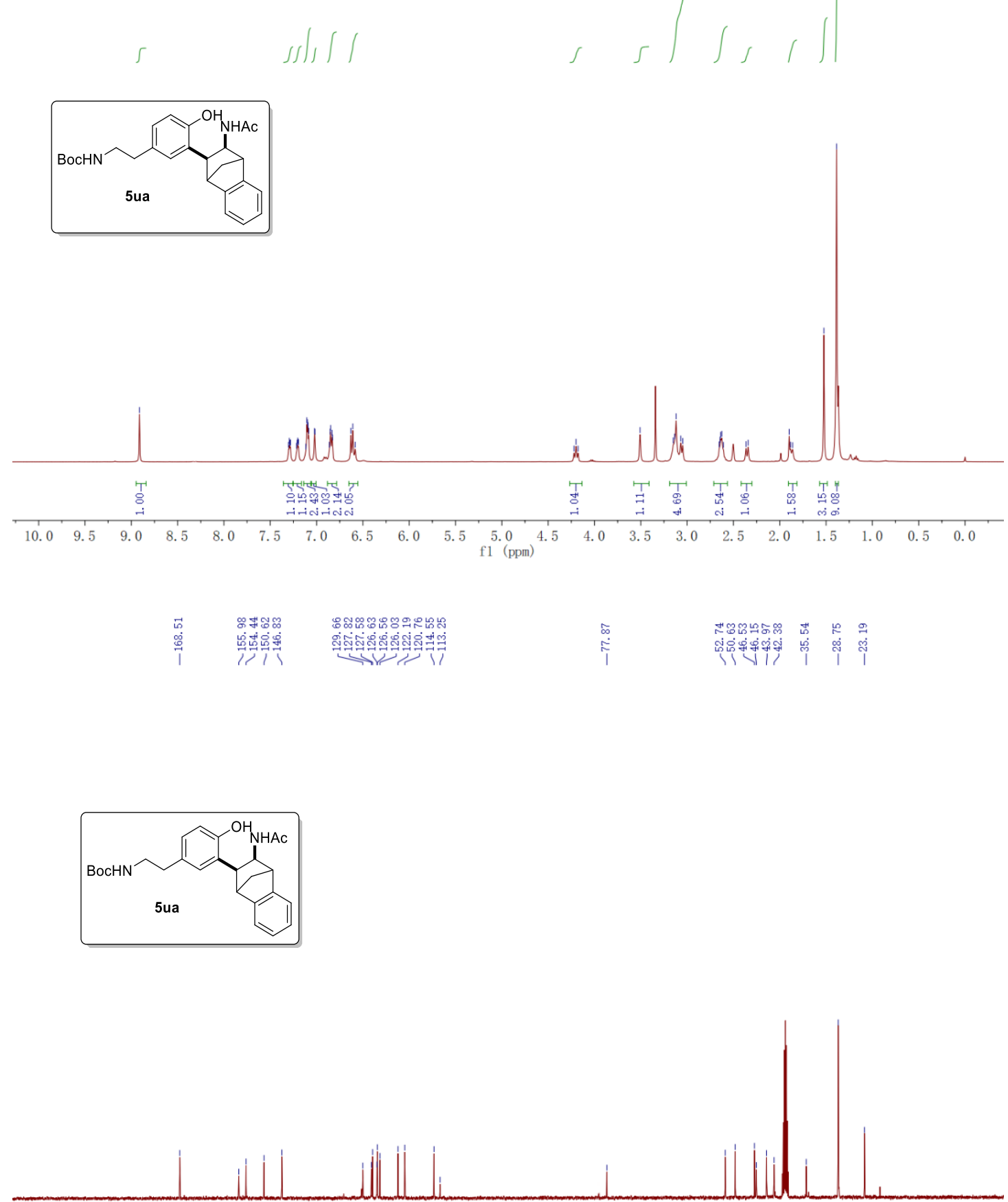

$\begin{array}{lllllllllllllllllllll}200 & 190 & 180 & 170 & 160 & 150 & 140 & 130 & 120 & 110 & 100 & 90 & 80 & 70 & 60 & 50 & 40 & 30 & 20 & 10 & 0\end{array}$ 


\section{${ }^{1} \mathrm{H}$ and ${ }^{13} \mathrm{C}$ NMR of $\mathbf{5 v a}$}

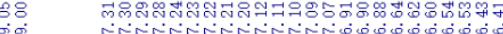

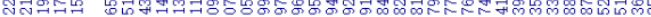

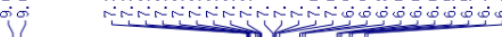
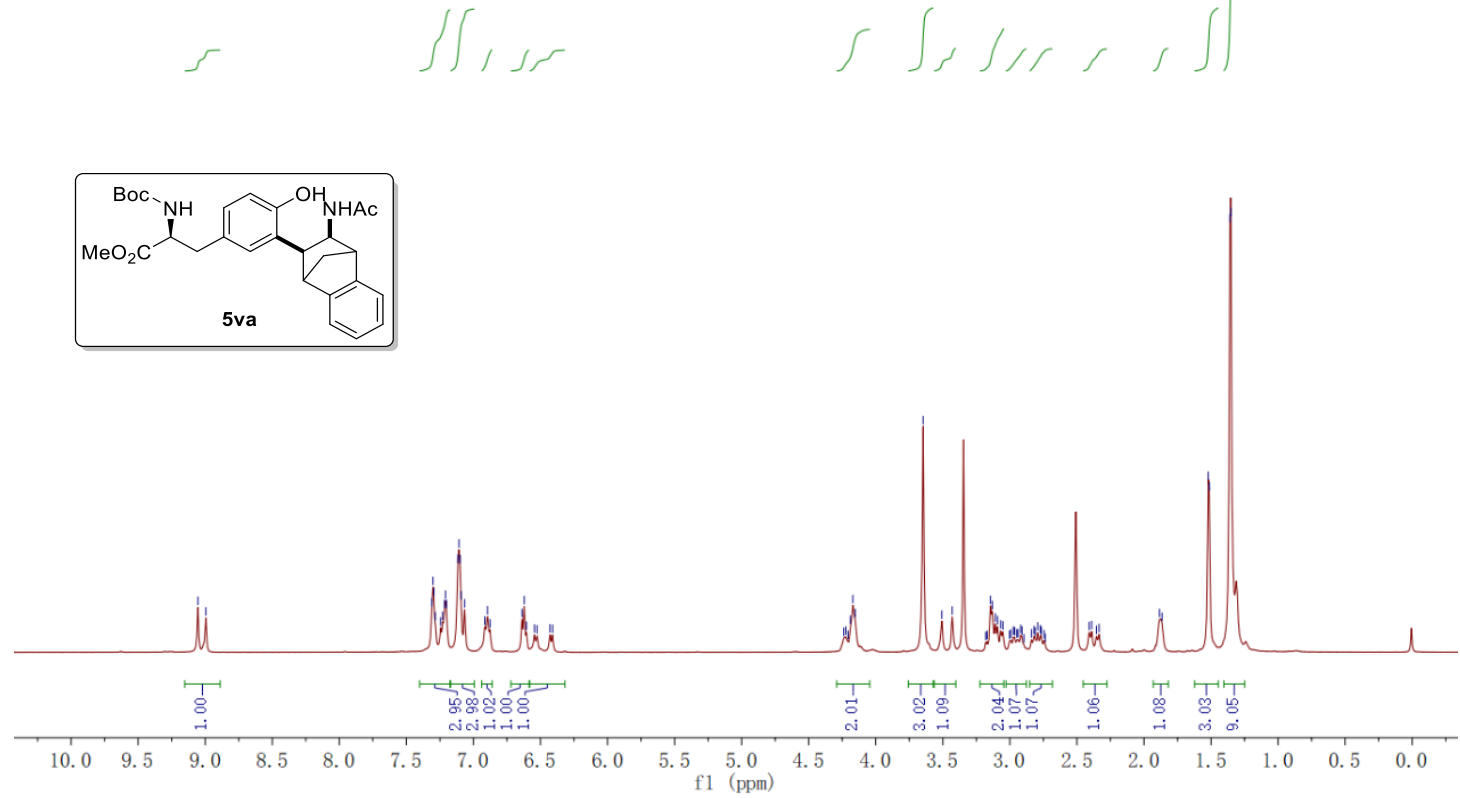

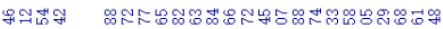

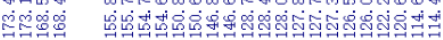

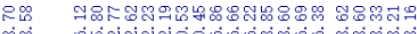

V.

Y

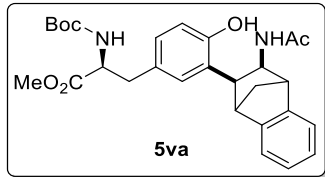

$\begin{array}{lllllllllll}200 & 190 & 180 & 170 & 160 & 150 & 140 & 130 & 120 & 110 & \begin{array}{l}100 \\ \mathrm{f} 1(\mathrm{ppm})\end{array}\end{array}$
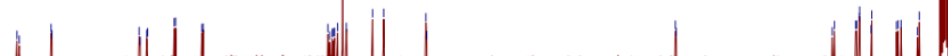


\section{${ }^{1} \mathrm{H}$ and ${ }^{13} \mathrm{C}$ NMR of $\mathbf{5 w a}$}

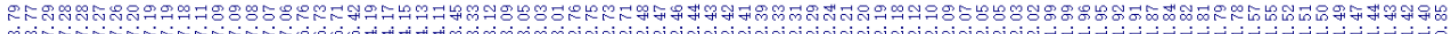

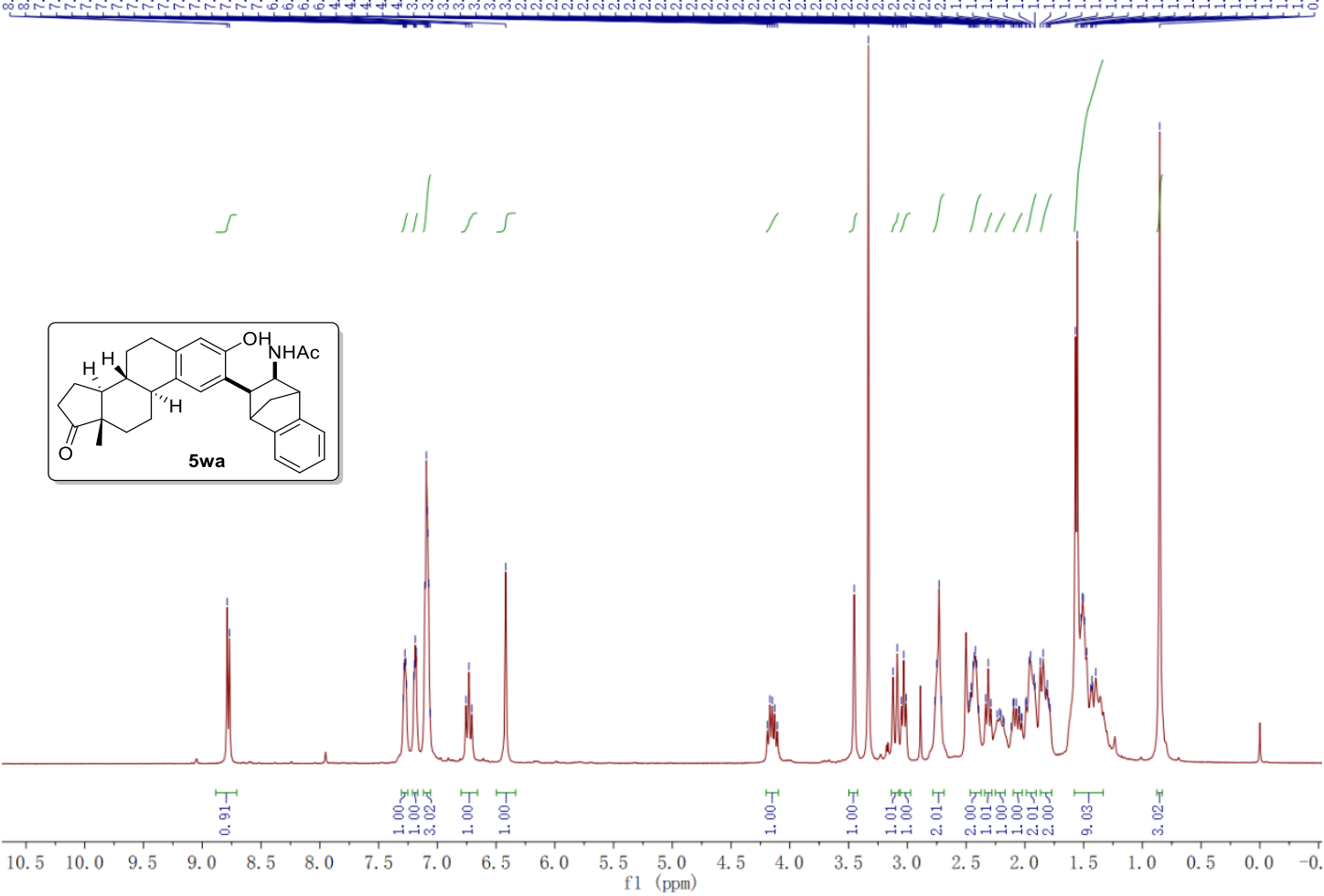

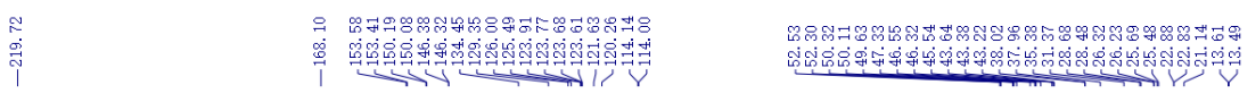
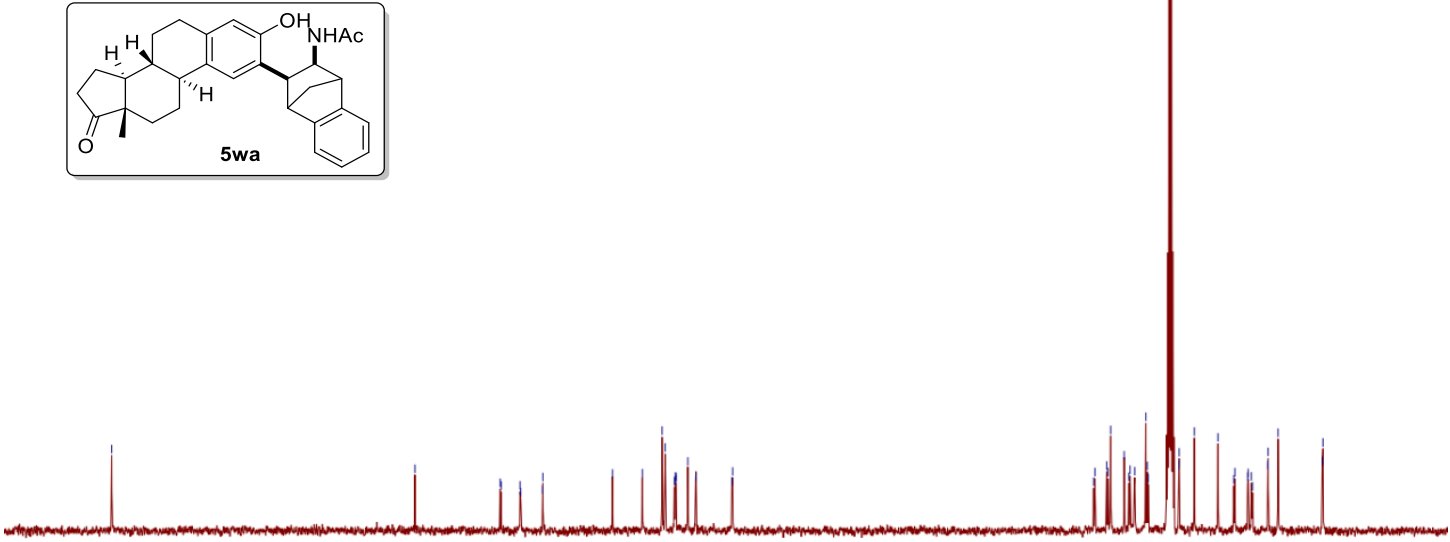

$\begin{array}{lllllllllllllllllllllllllllllll}230 & 220 & 210 & 200 & 190 & 180 & 170 & 160 & 150 & 140 & 130 & 120 & 110 & 100 & 90 & 80 & 70 & 60 & 50 & 40 & 30 & 20 & 10 & 0\end{array}$ 


\section{${ }^{1} \mathrm{H}$ and ${ }^{13} \mathrm{C}$ NMR of $\mathbf{5 a b}$}

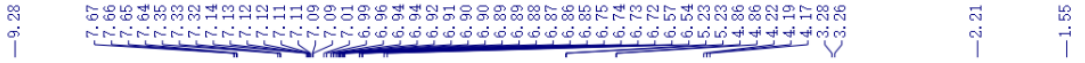
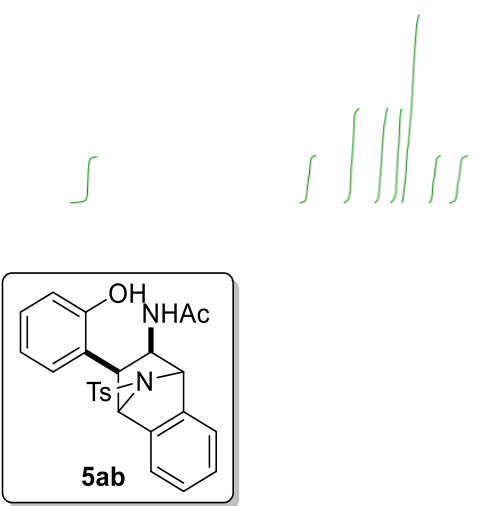

$5 a b$
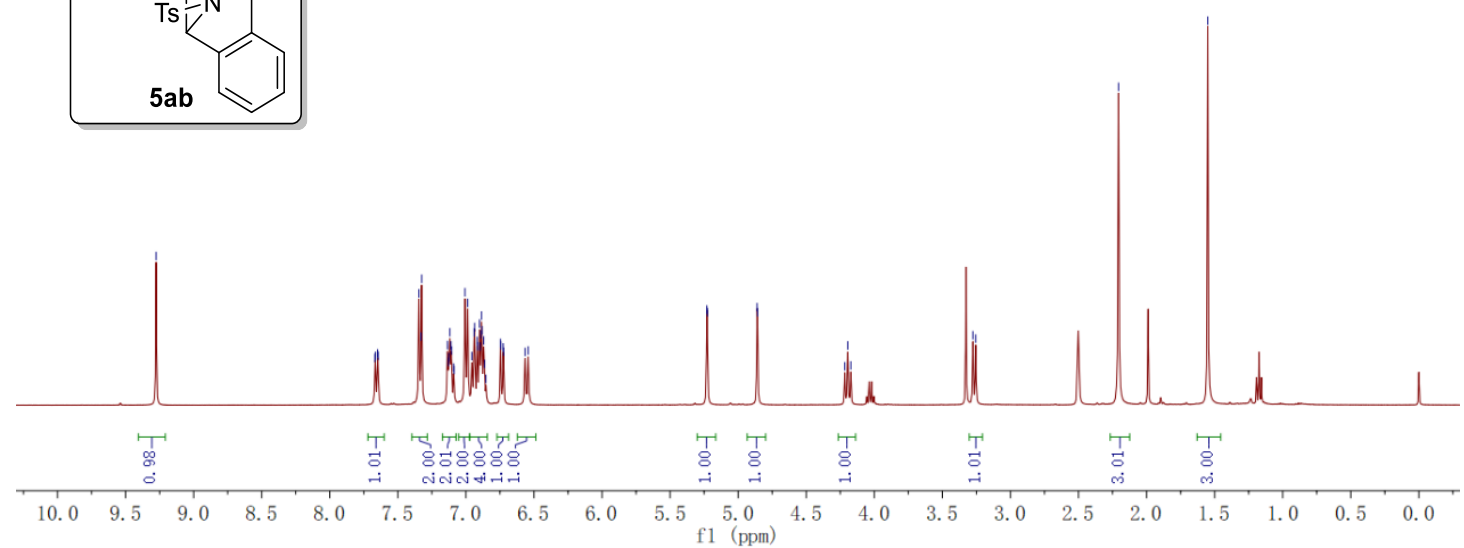

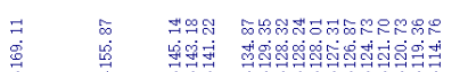

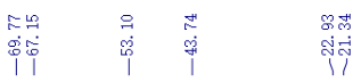
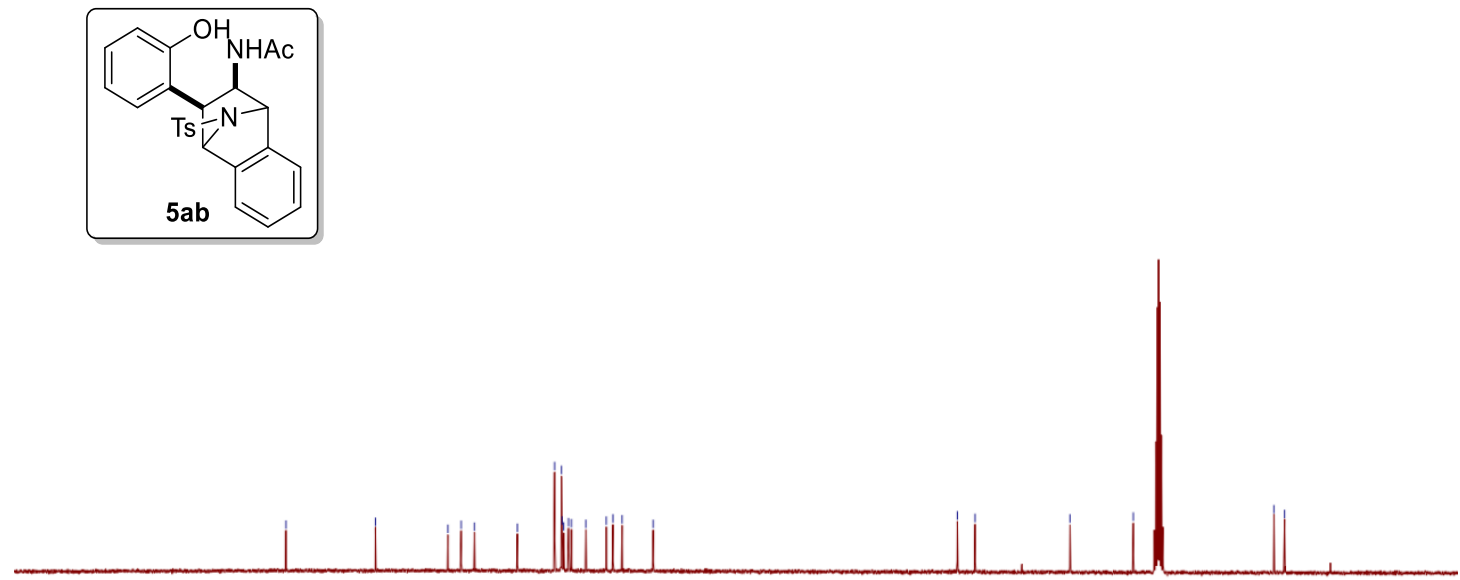

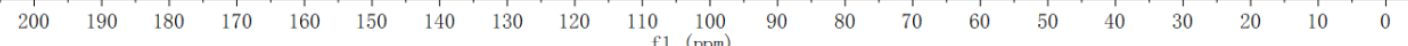


${ }^{1} \mathrm{H}$ and ${ }^{13} \mathrm{C}$ NMR of $\mathbf{5 a c}$
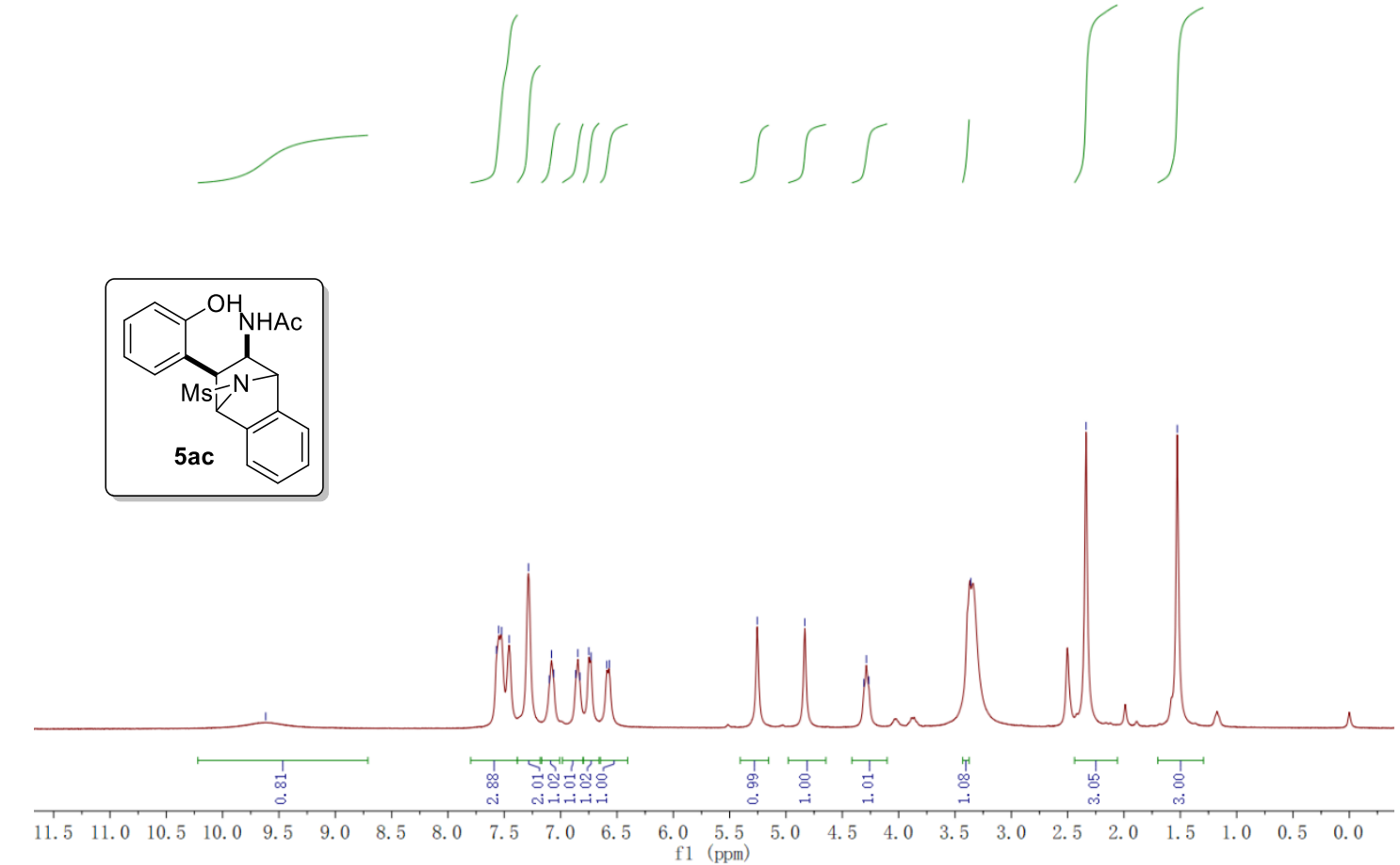

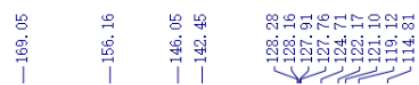
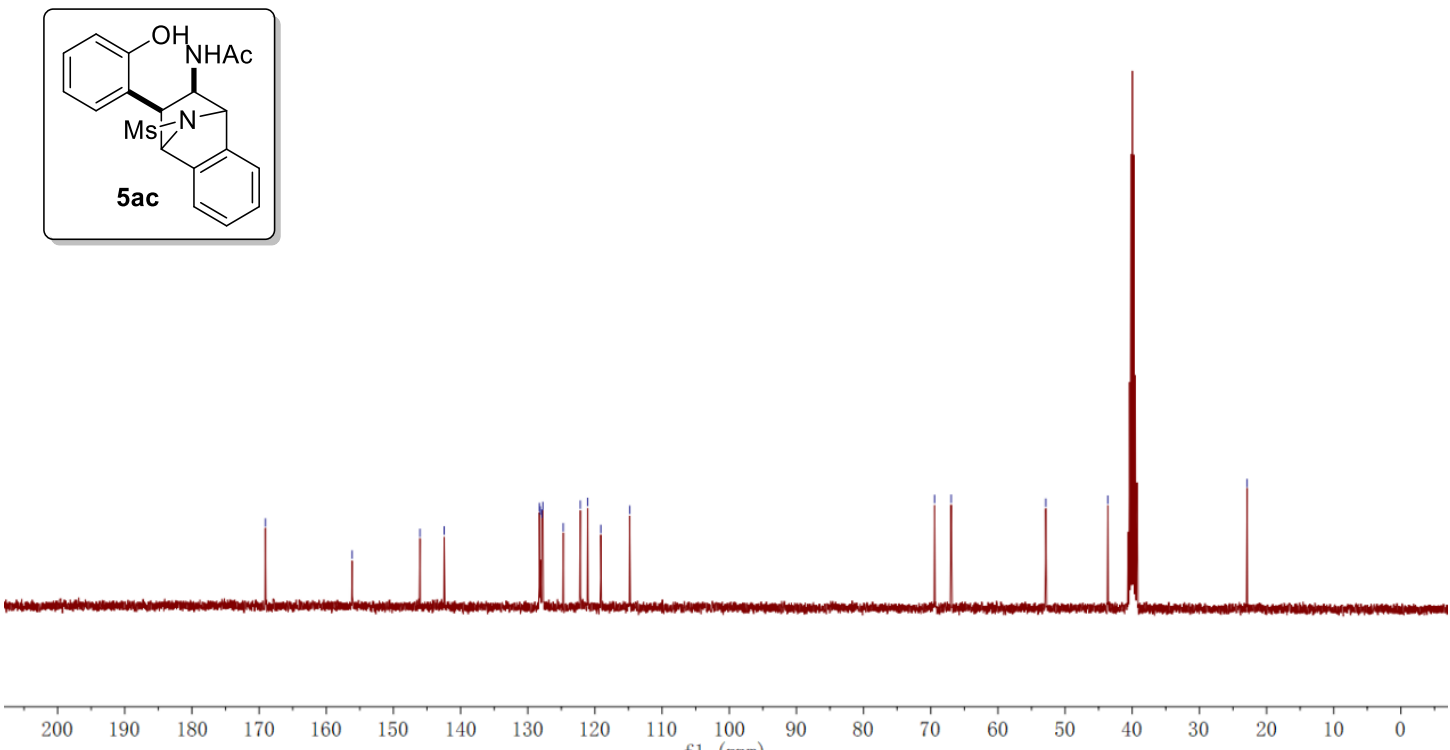


\section{${ }^{1} \mathrm{H}$ and ${ }^{13} \mathrm{C}$ NMR of $\mathbf{5 a d}$}

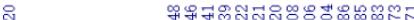

inivinivivinisiogo

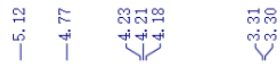

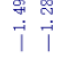

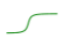

$\| / 1 /$
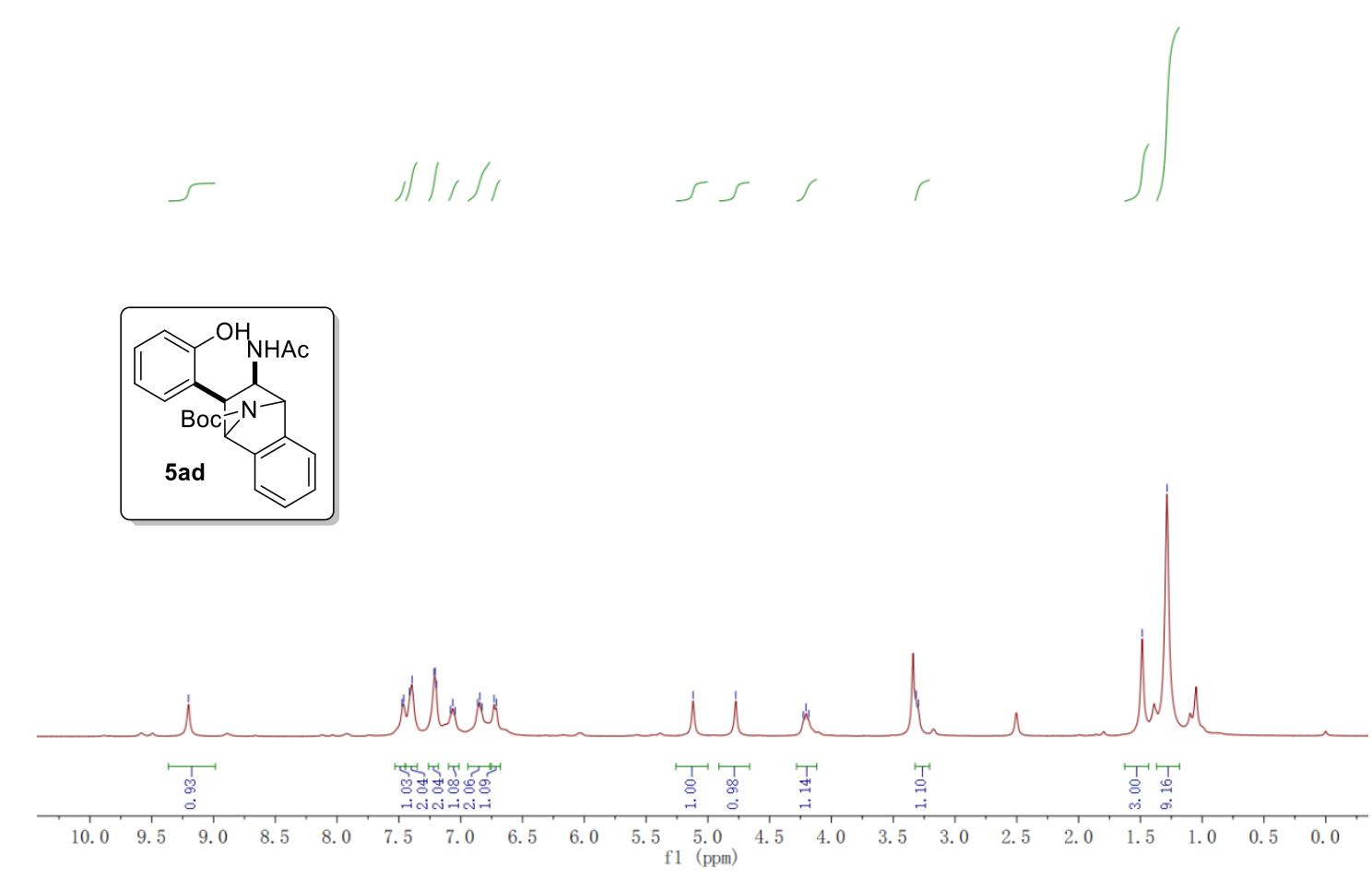

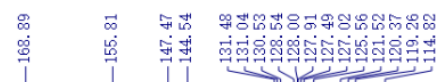
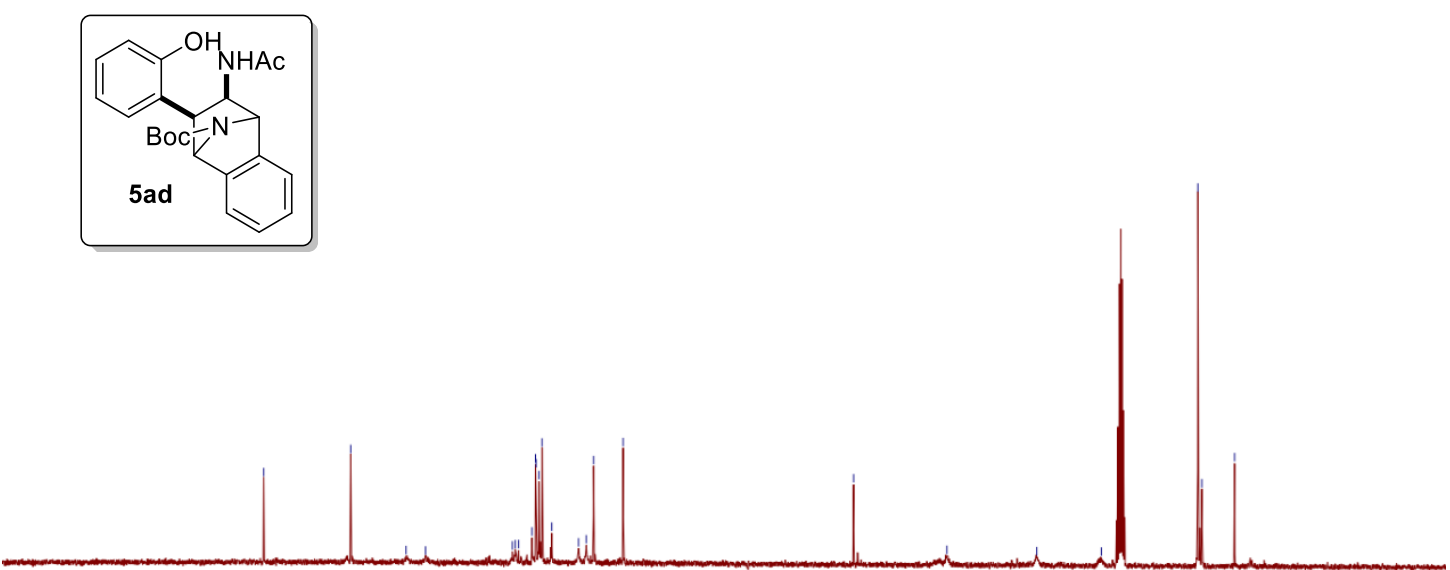

$\begin{array}{lllllllllllllllllllllll} & 200 & 190 & 180 & 170 & 160 & 150 & 140 & 130 & 120 & 110 & 100 & 90 & 80 & 70 & 60 & 50 & 40 & 30 & 20 & 10 & 0 & 10\end{array}$ 


\section{${ }^{1} \mathrm{H}$ and ${ }^{13} \mathrm{C}$ NMR of 5 ae}

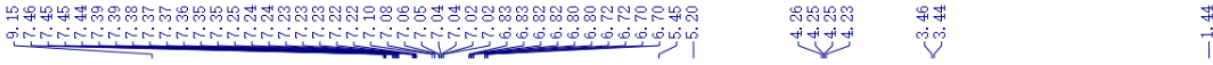
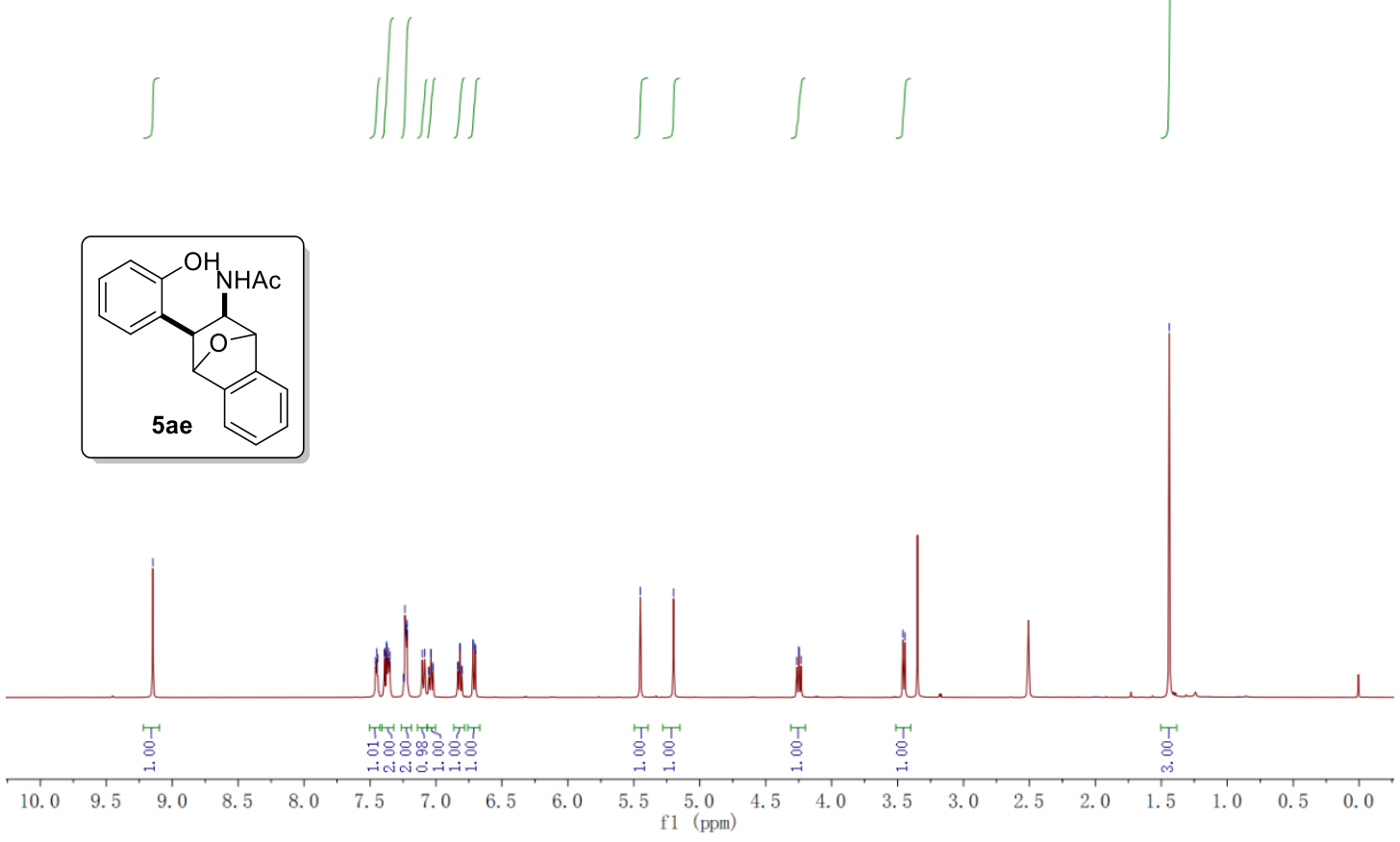

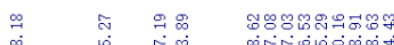

।
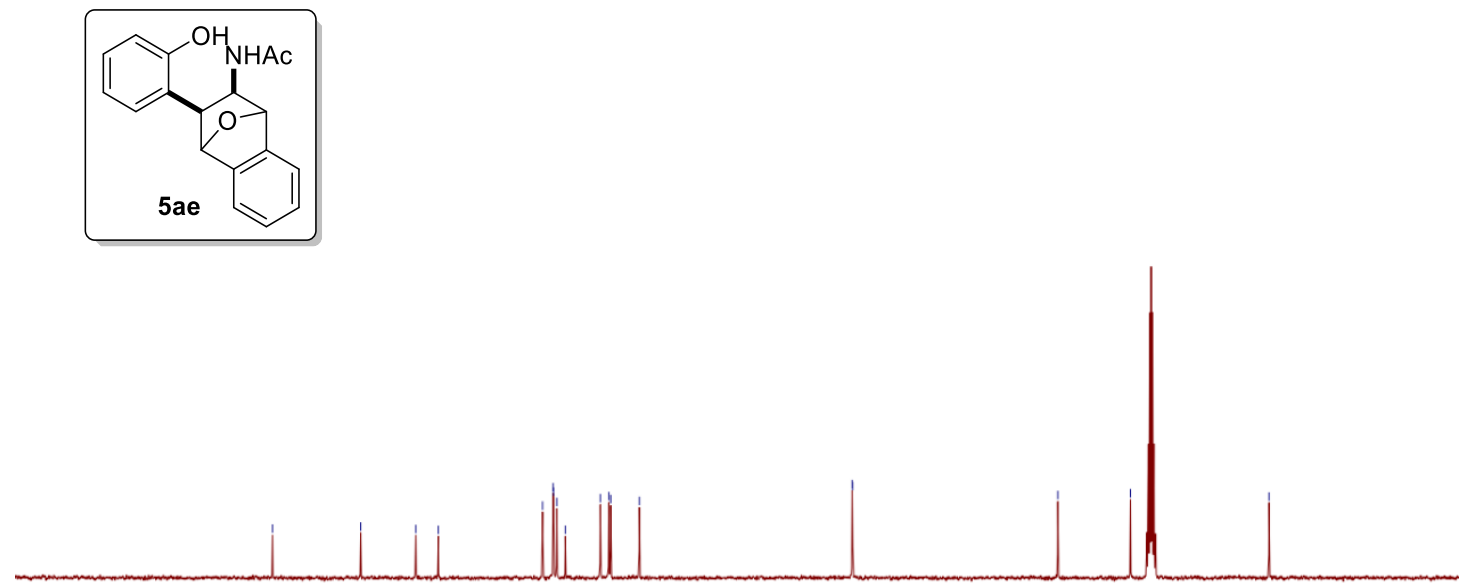

$\begin{array}{lllllllllllllllllllllll}200 & 190 & 180 & 170 & 160 & 150 & 140 & 130 & 120 & 110 & 100 & 90 & 80 & 70 & 60 & 50 & 40 & 30 & 20 & 10 & 0\end{array}$ 
${ }^{1} \mathrm{H}$ and ${ }^{13} \mathrm{C}$ NMR of $\mathbf{5 a f}$

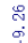

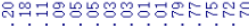

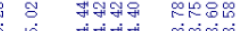

$\stackrel{i}{i}$

5

$\int / / \int$

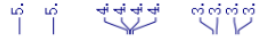

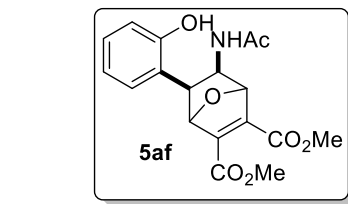

in

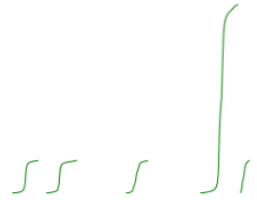

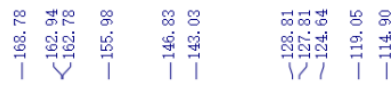

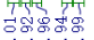

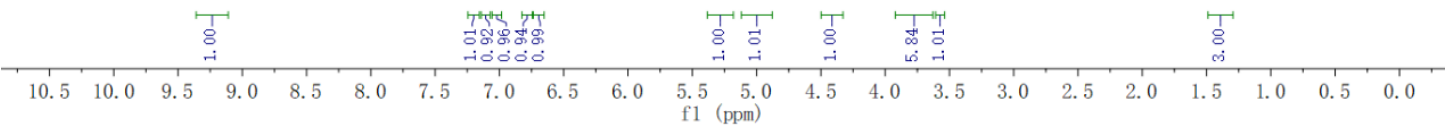

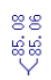

का

$\overrightarrow{0}$
ปู
I

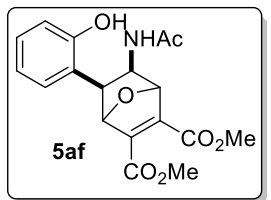

$\begin{array}{lllllllllllllllllllll}200 & 190 & 180 & 170 & 160 & 150 & 140 & 130 & 120 & 110 & 100 & 90 & 80 & 70 & 60 & 50 & 40 & 30 & 20 & 10 & 0\end{array}$ 
${ }^{1} \mathrm{H}$ and ${ }^{13} \mathrm{C}$ NMR of $\mathbf{5 a g}$

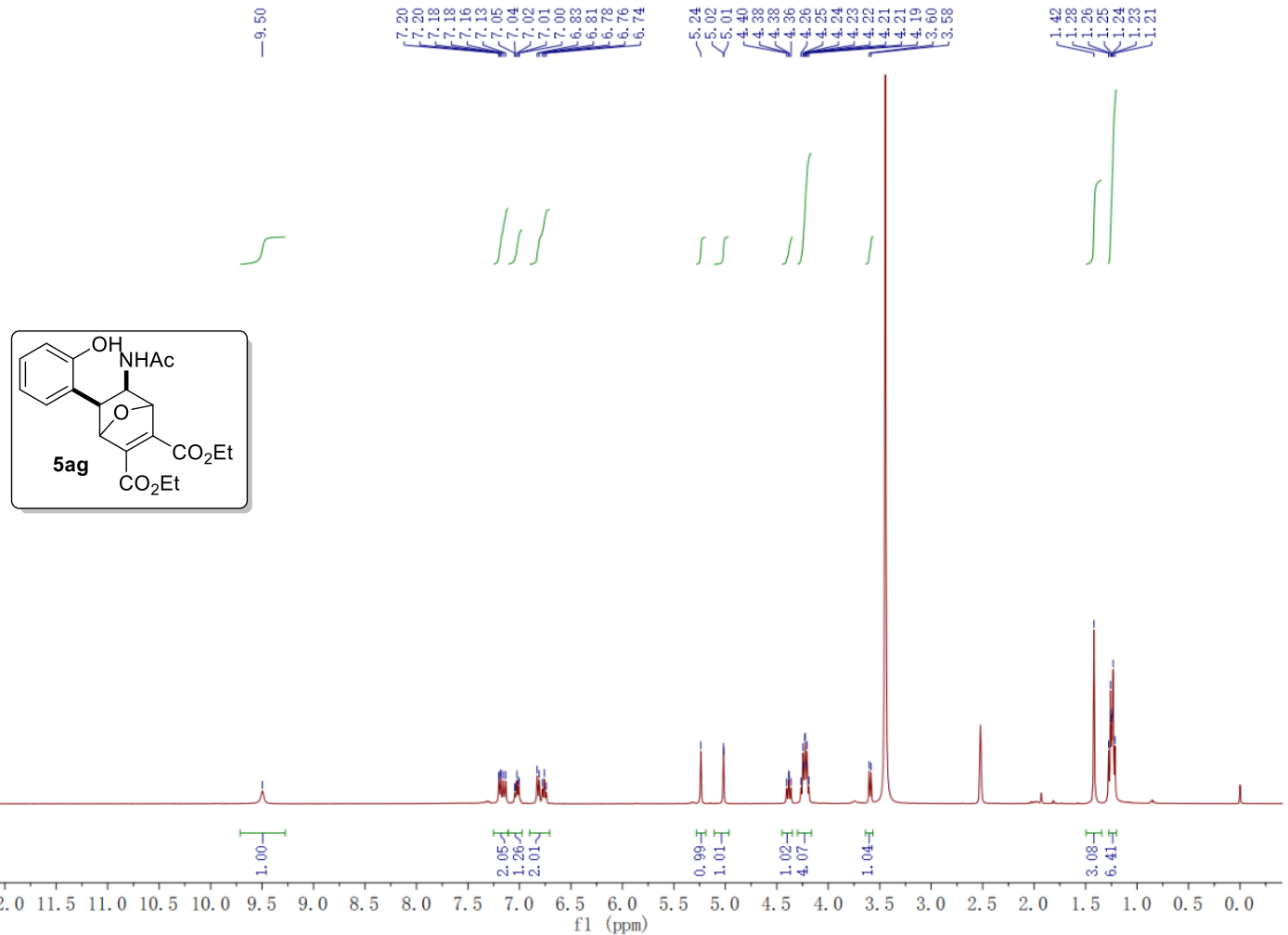

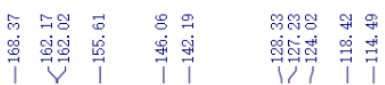

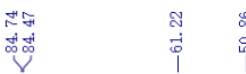

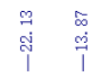

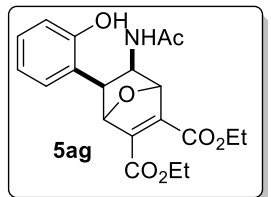

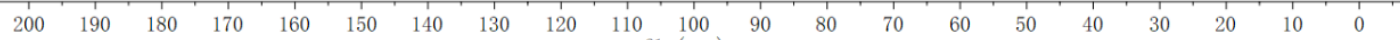


${ }^{1} \mathrm{H}$ and ${ }^{13} \mathrm{C}$ NMR of $\mathbf{5 a h}$
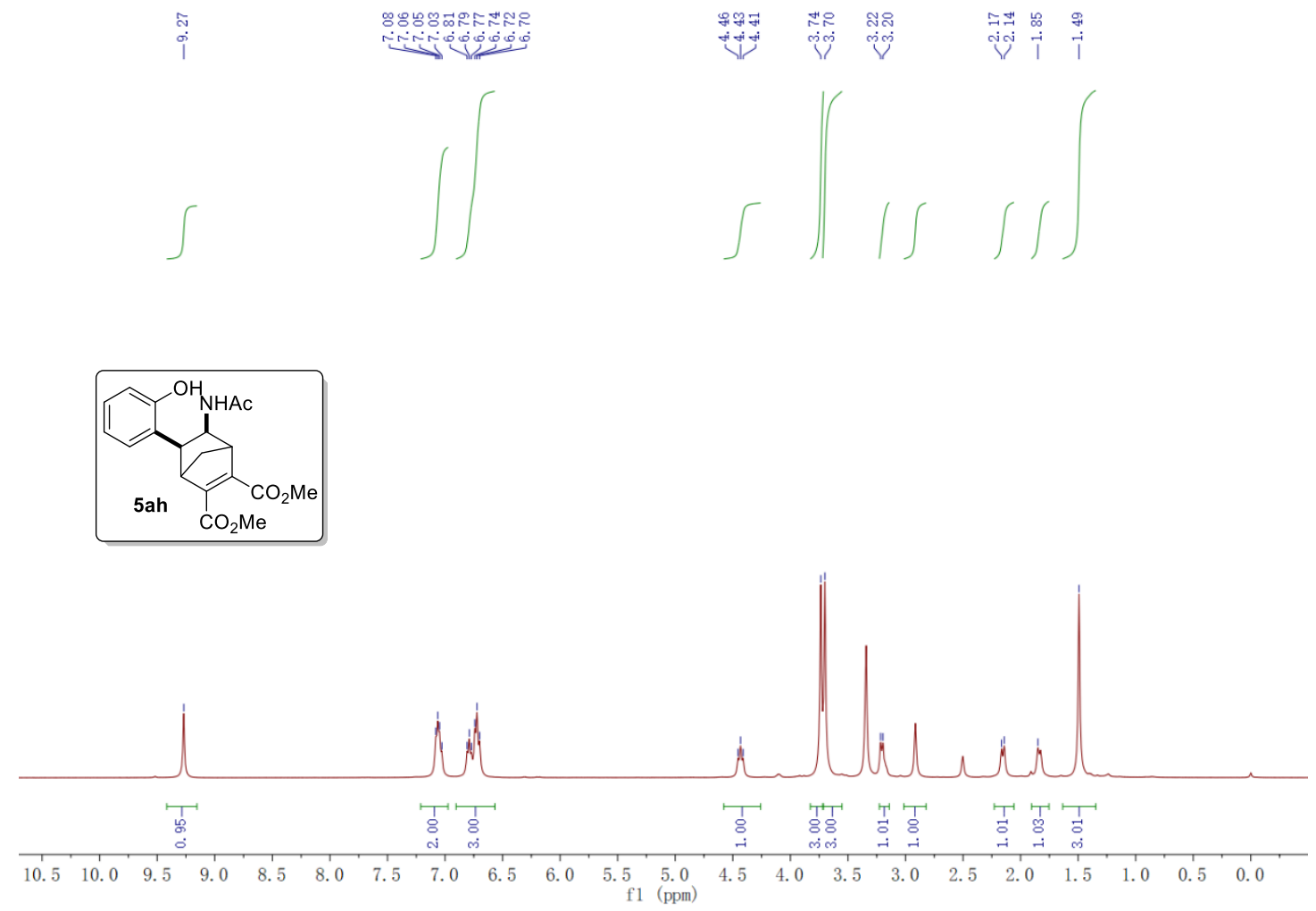

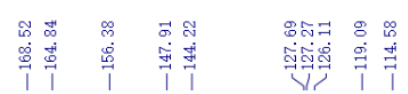

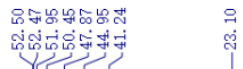
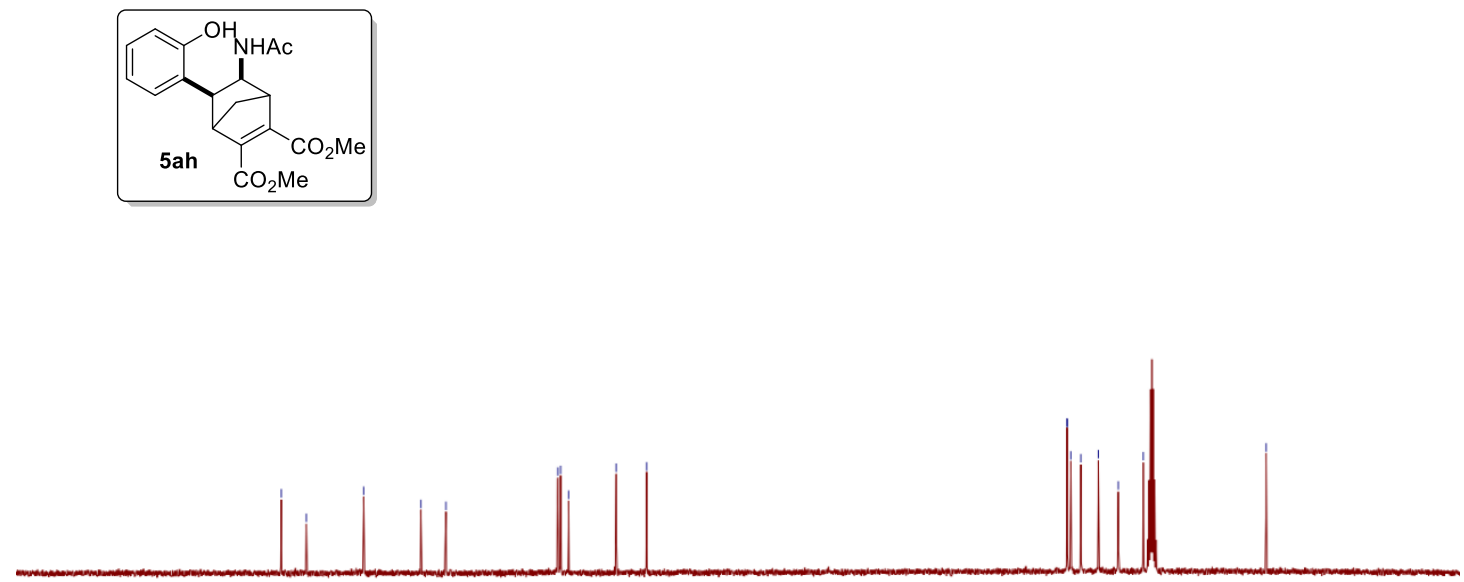

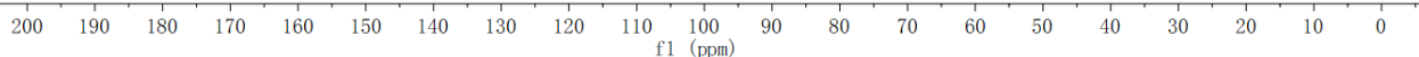

